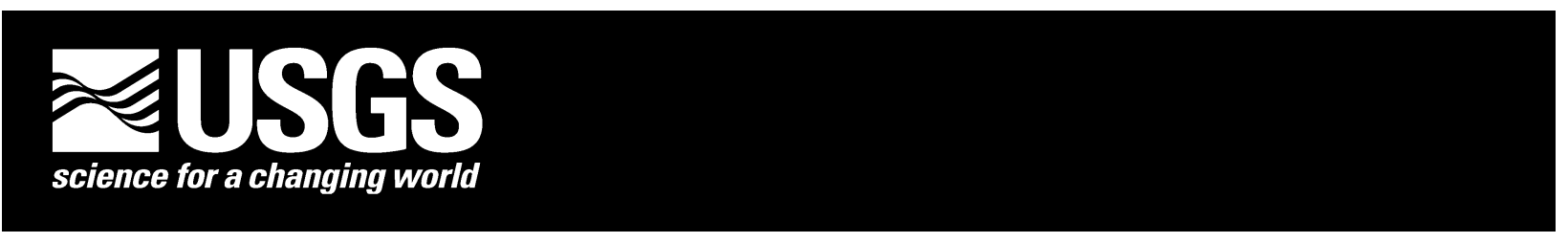

\title{
Geophysical Terranes of the Great Basin and Parts of Surrounding Provinces
}

By Jonathan M.G. Glen, Edwin H. McKee, Steve Ludington, David A. Ponce, Thomas G. Hildenbrand, and Melanie J. Hopkins

Open-File Report 2004-1008

2004

U.S. Department of the Interior

U.S. Geological Survey

This report is preliminary and has not been reviewed for conformity with U.S. Geological Survey editorial standards or with the North American Stratigraphic Code. Any use of trade, firm, or product names is for descriptive purposes only and does not imply endorsement by the U.S. Government.

${ }^{1}$ U.S. Geological Survey, 345 Middlefield Road, MS 989 Menlo Park, CA 94025 


\section{TABLE OF CONTENTS}

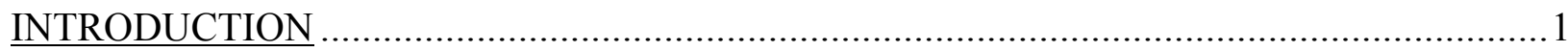

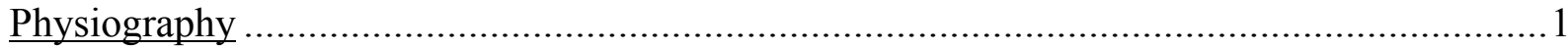

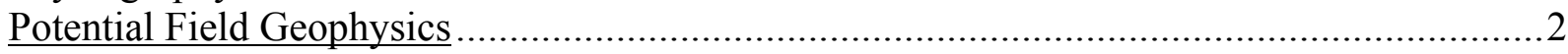

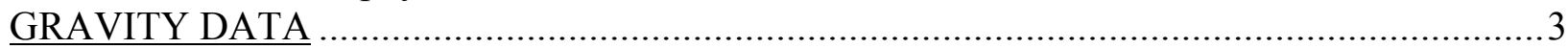

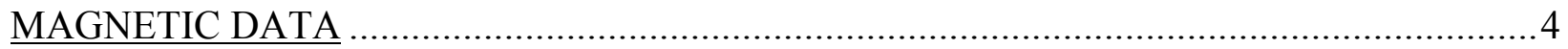

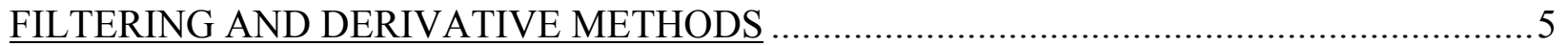

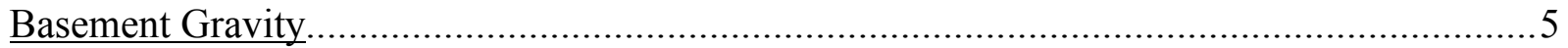

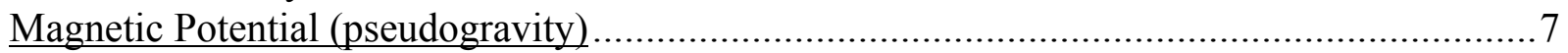

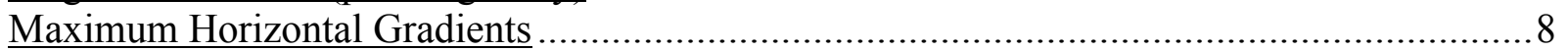

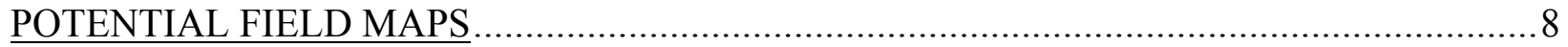

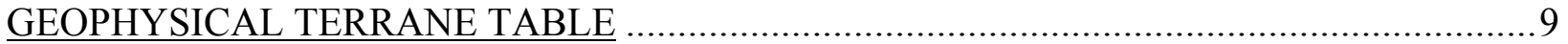

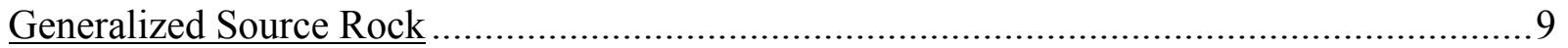

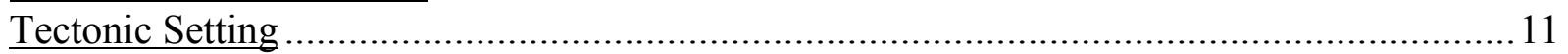

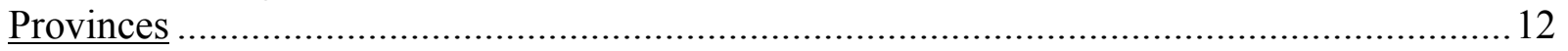

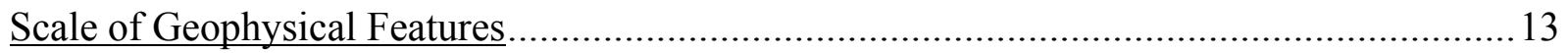

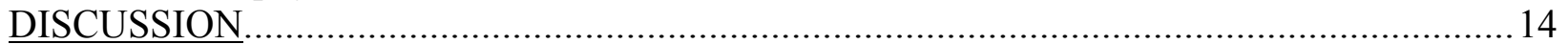

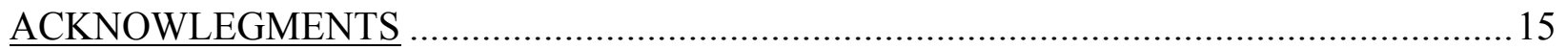

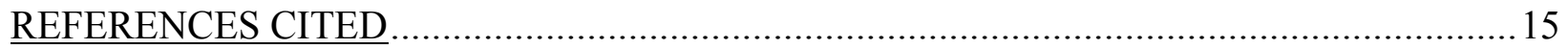

\section{FIGURES}

Figure 1 Physiographic province map of the Great Basin.

2 Basement gravity terrane map of the Great Basin.

3 Magnetic potential (pseudogravity) terrane map of the Great Basin.

4 Regional isostatic gravity and pseudogravity field maps of the Great Basin.

\section{TABLES}

Table $\quad 1$ Geophysical features of the Great Basin and parts of surrounding provinces.

2 Physiographic provinces and their geologic and geophysical character.

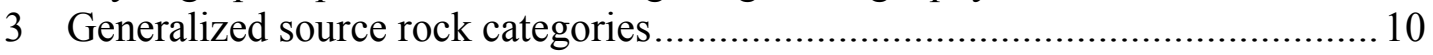

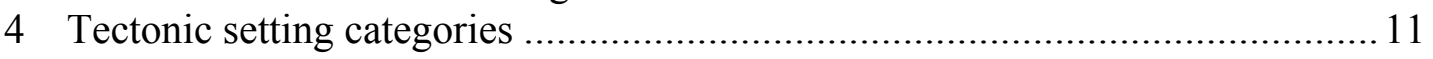




\section{INTRODUCTION}

This study of geophysical terranes within and surrounding the Great Basin of the western United States (fig. 1A) integrates geophysical and geologic data to provide new insights on basement composition and structure at local, intermediate, and regional scales. Potential field (gravity and magnetic) studies are particularly useful to define the location, depth, and extent of buried basement sources and fundamental structural or compositional boundaries. They especially serve in imaging the subsurface in areas of extensive Cenozoic cover or where surface outcrops may be detached from the deeper crust. Identifying buried compositional or structural boundaries has applications, for example, in tectonic and earthquake hazard studies as they may reflect unmapped or buried faults. In many places, such features act as guides or barriers to fluid or magma flow or form favorable environments for mineralization and are therefore important to mineral, groundwater, and geothermal studies. This work serves in assessing the potential for undiscovered mineral deposits and provides important long-term land-use planning information. The primary component of this report is a set of geophysical maps with anomalies that are labeled and keyed to tables containing information on the anomaly and its source. Maps and data tables are provided in a variety of formats (tab delimited text, Microsoft Excel, PDF, and ArcGIS) for readers to review and download. The PDF formatted product allows the user to easily move between features on the maps and their entries in the tables, and vice-versa. Our goal in highlighting these anomalies is to stimulate thought and research about crustal features of the Great Basin. While we do not offer comprehensive interpretation of every gravity and magnetic feature in the Great Basin, we hope this product will serve as a useful spatial catalog of those features.

\section{Physiography}

The Great Basin is a broad hydrologically closed region spanning much of the western United States (fig. 1A). It forms part of the Basin and Range Province (Figure 1B), which is characterized by late Cenozoic regional extension ranging from Mexico nearly to Canada and California to Texas. This extension has been accompanied by normal and detatchment faulting, crustal block uplift, downdrop, rotations and tilting, crustal thinning and ductile flow, and 
widespread magmatism. Crustal blocks expose a range of variably deformed pre-Cenozoic rocks as well as early and middle Cenozoic volcanic rocks.

The study area (fig. 1A) includes the Great Basin and parts of ten adjacent physiographic provinces (Cascade Mtns, Columbia Plateaus, Snake River Plain, Northern Rocky Mtns, Sierra Nevada, Great Valley, Coast Ranges, Mojave Desert, Colorado Plateaus, and Middle Rocky Mtns). The Great Basin is sharply defined on its northern, western and eastern margins where it is bound by the Snake River Plain, Sierra Nevada, and Colorado Plateaus, respectively. Northwestern, northeastern and southern boundaries, however, are less well defined. Definitions of the Great Basin and its surrounding provinces used in this report largely follow those of Fenneman and Johnson (1946).

\section{Potential Field Geophysics}

Several excellent reviews of magnetic methods have been published by Grant (1985a,b), Reford (1980), Hinze and Zietz (1985), Blakely (1995), and Gunn and Dentith (1997). Similarly, Simpson and others (1986), Simpson and Jachens (1989), Jachens and others (1989), and Blakely (1995) provide informative reviews of gravity methods. These geophysical techniques, which allow imaging of subsurface structure, are particularly important in the Great Basin where more than $75 \%$ of the surface is covered by Cenozoic deposits. Gravity and magnetic anomalies occur due to lateral contrasts in rock density and magnetic properties (induced and remanent magnetizations), respectively. Rock-property contrasts may occur within a rock unit, such as resulting from gradual lateral facies changes or heterogeneous alteration, or at geologic structures such as faults, folds, or contacts. The geometry and depth to sources, the character of the geomagnetic field, and the rock properties of sources all determine the character of the associated potential field anomalies. Despite the complexity of potential fields and their sources, gravity and magnetic data can be used to resolve the geometry and origin of sources, particularly when combined with other geologic constraints such as the regional tectonic models, surface geology, and seismic data. 


\section{GRAVITY DATA}

Gravity data were compiled from a variety of sources reduced and gridded (Hildenbrand and others, 2000) to produce the various gravity and derivative maps shown in this report. Gravity maps, derived from these data, reflect anomalies that may arise from contrasts in density due to contacts between different rock units, partial melting, or phase transitions. Generally, longwavelength anomalies with smooth gradients originate from sources at depths greater than sources of short-wavelength anomalies that display steep gradients. While short-wavelength anomalies must arise from sources at shallow depths, long-wavelength anomalies, could arise from shallow, thin sources that have gently sloping sides.

In order to produce a gravity map reflecting lateral variations in density in the crust, raw gravity measurements were reduced using standard gravity reduction methods (Dobrin and Savit, 1988; Blakely, 1995). These reductions remove the effects of elevation, topography, total mass, rotation, and ellipsoidal shape of the Earth and yield the complete Bouguer gravity anomaly (CBA). Although the CBA reveals lateral density variations at short wavelength scales, it does an inferior job isolating longer wavelength features since these are often masked by broad anomalies due to deep crustal roots that isostatically compensate topographic loads. The isostatic correction attempts to remove effects of compensating masses.

Despite its name, an isostatic anomaly does not necessarily reflect the state of isostatic equilibrium. In this study, we are most interested in those cases where anomalies arise from density inhomogeneities in the crust. Nonetheless, it should be noted that an isosatic anomaly may reflect areas out of isostatic balance, either dynamically or by means of elastic support. For example, because the isostatic correction ignores effects of lithospheric strength it may not acurately account for regional fields associated with topographic loads. Thus, in areas where the lithoshere is exceptionally strong, topographic loads can be supported regionally, and compensation distributed over the area of elastic flexure rather than being locally compensated.

A general correlation between topography and the regional bouguer gravity field indicates that across the Great Basin the crust is in isostatic equilibrium. A common misconception is that the depth of compensation corresponds to the Moho. Because Bouguer gravity does not correlate to crustal thickness (Eaton and others, 1978) and gravity effects due to variations in the crustal 
thickness versus lithospheric thickness differ by an order of magnitude (i.e., asthenoshere/lithosphere density contrast is $\sim 0.08 \mathrm{~g} / \mathrm{cc}$ and between crust and lithosphere it is $\sim 0.4 \mathrm{~g} / \mathrm{cc}$ ), compensation likely occurs at mid-crustal depths. In the Great Basin, where heat flow is high and extension is large, isostatic compensation is probably achieved over relatively short lateral distances (e.g. $150 \mathrm{~km}$ ), and within relatively shallow crustal depths perhaps within the upper $20 \mathrm{~km}$ (Eaton and others, 1978).

Although the close correspondence of topography and Bouguer gravity suggests compensation is achieved largely by an Airy-Heiskanen mechanism, some regions, such as the Rocky Mountains (Eaton and others, 1978, Woollard, 1972), are best fit with a Pratt model that achieves compensation through lateral density variations. This is indicated by crustal density variations inferred from seismic compressional wave velocity data. It has been suggested that isostatic equilibrium and extension in the Great Basin are likely accommodated by shallow crustal intrusion or ductile flow, especially in areas such as core complexes where the crust has undergone significant stretching and thinning (Thompson and McCarthy, 1990).

\section{MAGNETIC DATA}

Magnetic data were derived from a compilation of statewide compilations (Hildenbrand and others, 2000). Surveys have all been continued to a common reference level of $0.305 \mathrm{~km}$ above ground surface, gridded, and filtered to produce the magnetic maps shown in this report. Variations in the magnetic field arise from contrasts in the magnetic properties of rocks. These contrasts can be due to a number of different sources including crustal structures, metamorphism and alteration, variations in remanent magnetization, and variations in the concentration and type of magnetic minerals.

Generally, the most significant contributions to crustal anomalies arise from magnetite, one of the most common, strongly magnetic minerals in the crust. Furthermore, because the magnetic susceptibility of magnetite dramatically drops at temperatures above the Curie point $\left(580^{\circ}\right.$ for pure magnetite), the portion of crust most responsible for variations in the magnetic field is that 
which lies above the Curie isotherm. This probably coincides roughly with the Moho, though in areas of high heat flow, like the Great Basin, it may occur at significantly shallower depths.

Although the magnetic field strength depends on both induced and remanent crustal magnetization, it is often assumed that the remanent component is negligible. This is because remanence is often low enough to ignore or because remanent components are often aligned close to the induced field component. In general, this is supported by the character of many magnetic anomalies. For example, in the northern hemisphere, anomalies often have relatively weak minima that lie to the north of their maxima counterparts (note that magnetic sources generally display bipolar anomalies). An important effect on the character of geophysical anomalies is the depth to the source. The shallower the depth to a body, the higher the amplitude, the shorter the wavelength, and the sharper the gradients of its anomaly. Generally, magnetic highs arise from mafic igneous and crystalline basement rocks, whereas lows arise from felsic igneous, sedimentary, or altered basement rocks. Igneous outcrops not associated with high-amplitude magnetic anomalies might be thin or contain low concentrations of primary magnetic minerals, or have lost them due to alteration.

Aeromagnetic anomalies in most of the Great Basin have been found to arise from Precambrian metamorphic, Mesozoic granitic and gabbroic, Tertiary calc-alkaline volcanic and intrusive, and Tertiary basaltic rocks (Blakely, 1988). Much of the Precambrian basement in the Great Basin is weakly magnetic, in contrast to the magnetic basement of the Colorado Plateaus (Mabey and others, 1978).

\section{FILTERING AND DERIVATIVE METHODS}

\section{Basement Gravity}

An iterative gravity inversion method (Jachens and Moring, 1990), used to determine the depth to pre-Cenozoic basement and the thickness of Cenozoic basin deposits, was applied to the Great Basin to obtain a basement gravity map (Figure 2), a by-product of the depth-to-basement

process. Basement gravity, which is the isostatic gravity with the effects of Cenozoic basins 
removed, reflects lateral density variations in pre-Cenozoic basement rocks and is particularly useful for defining pre-Cenozoic structures and crustal geophysical terranes.

Isostatic gravity anomalies were used during this inversion process because they enhance or reflect shallow- to mid-crustal sources within the Earth by removing long-wavelength variations in the gravity field inversely related to regional topography (Simpson and others, 1986). The Basement gravity inversion process depends on their being a significant contrast in density between the usually dense basement rocks and any overlying Cenozoic deposits. While this is true for much of the Great Basin, Cenozoic mafic volcanic rocks can have densities similar to those of their underlying basement rocks. This is a problem, particularly in the northwesternmost part of the Great Basin that is blanketed by middle Miocene mafic lava flows. As a result, the inversion process was not applied in areas where the thickness of overlying volcanic rocks could not be determined. The boundary within which the depth-to-basement calculation was applied is shown in Figure 2. Outside this boundary, we show isostatic gravity values.

The depth-to-basement method separates the gravity field into two components: the gravity field caused by pre-Cenozoic basement and the gravity field caused by overlying younger basin deposits. An initial basement gravity field is determined by using only stations located on preCenozoic basement outcrops. The initial basement gravity field is approximate because stations located on basement are influenced by the gravity effect of low-density deposits in nearby basins, especially for those stations near the edge of the basins. The difference between the isostatic gravity and basement gravity fields provides the first estimate of the basin gravity field, which is inverted to provide the first estimate of the basin shape. The gravitational effect of the basins is subtracted from each station located on basement and a new and improved basement gravity field is determined. This process is repeated until successive iterations converge. Inversion of the final basin gravity field yields the final estimate of the depth to pre-Cenozoic basement. The density of basement rocks is allowed to vary horizontally, whereas the density of basin-filling deposits increases with depth according to a density-depth relationship defined by Jachens and Moring (1990).

A number of limitations are inherent in this method, including uncertainties that relate to: the gravity data coverage, especially for stations on basement outcrops; the density-depth function; accuracy and scale of the geologic mapping; simplifying assumptions regarding concealed 
geology; and the distribution of basement outcrops. A more detailed discussion of the limitations and accuracy of the method were provided by Jachens and Moring (1990).

\section{Magnetic Potential (pseudogravity)}

Crustal magnetism differs from and is more complex than gravity, which varies due simply to the crustal density distribution. Magnetism varies because of differences in both the concentration and type of magnetic minerals within the crust (analogous to the relation between density and gravity), and crustal remanent magnetization. Furthermore, because crustal magnetization is seldom vertical, except at the magnetic poles, anomalies are asymmetric and not centered over their sources. In addition, unlike gravity, crustal remanent magnetism has a depth limit set by the Curie temperature isotherm, the temperature above which remanent magnetization does not exist. Magnetic data also tend to highlight shallower features than gravity, because magnetic field strength attenuates more significantly with distance to the source than does gravity.

Because of this complexity of magnetic anomalies they are typically more difficult to interpret. The pseudogravity or magnetic potential transformation (Baranov, 1957; Blakely, 1995) removes asymmetry of anomalies, by centering them over their sources, and allows for a more accurate estimate of the extent of source bodies. In addition, it helps highlight regional magnetic features masked by high-frequency anomalies.

Because the magnetic and gravity potentials are related by a directional derivative, thus the total magnetic field can be transformed into an equivalent gravity field. Magnetic potential, or pseudogravity, maps are produced by the transformation of the magnetic field into the equivalent gravity field assuming a density distribution equal to the magnetization distribution (Baranov, 1957). The ratio between magnetization and density is held constant and remanent magnetization is assumed to be either negligible or in the same direction as the Earth's magnetic field. This process amplifies long wavelengths (deeper sources) at the expense of short wavelengths (shallow sources). In addition, because gravity anomalies have their steepest gradients approximately over the edges of their causative sources, especially for shallow sources, the magnetic potential map can be used to approximate the edges of magnetic sources (Blakely, 1995). 


\section{Maximum Horizontal Gradients}

To better define the edges of geophysical sources and to help derive geophysical lineaments and terranes, the amplitudes of the maximum horizontal gradients (AMHG) of both gravity and magnetic data were computer generated. A technique described by Blakely and Simpson (1986) was used to calculate the AMHG. Because the AMHG tend to lie over the edges of bodies with near vertical boundaries (Cordell and McCafferty, 1989; Grauch and Cordell, 1987), they are useful at estimating the extent of buried sources. AMHG were derived for both previously described basement gravity and magnetic potential maps. Because these maxima reflect abrupt lateral changes in the density or magnetization of the underlying rocks, they were used to aid in defining the boundaries of geophysical terranes shown in Figures 2 and 3.

\section{POTENTIAL FIELD MAPS}

Gravity and magnetic lineations (shown in figs. 2 and 3, respectively) were derived with the aid of the AMHG method described above. Geophysical terranes are based in part on the AMHGderived boundaries and on geophysical fabric. Areas, for example, that display a consistent trend or wavelength of anomalies, in contrast to their surroundings, were defined as distinct geophysical terranes that may represented discrete crustal blocks having similar physical properties or sources. Gravity and magnetic terrane maps (figs. 2 and 3) were created by visual inspection of gravity, magnetic, and derivative geophysical maps, and by drawing polygons

around similar geophysical areas using derived lineaments as a guide to locating terrane boundaries. In addition to geophysical terranes, we have also included a number of lineations. A few of these are defined as geophysical features listed in table 1, while others occur on figures 2 and 3 simply as unlabeled features that are intended to highlight the geophysical fabric. 


\section{GEOPHYSICAL TERRANE TABLE}

The geophysical terrane table (table 1) lists geophysical terranes that occur on the gravity and magnetic terrane maps (figs. 2 and 3). The table is organized to allow the user to identify features in the table that occur on the maps, and to move between maps and table. Terrane names contain (from left to right): a two letter (uppercase) code identifying the state in which they mostly occur, a single letter (lowercase) code identifying whether the feature is a gravity (g) or magnetic (m) terrane, and a two digit number indicating the feature number. Geologic and geophysical references provided in the table point the user to an example of work pertaining to some part of the anomaly or to its presumed source rock, and should not be considered a complete list of pertinent or historical citations. We refer the reader to references contained within the cited publications for further background. Several of the table columns are specifically defined such that they may be used as search terms or as tools for sorting the table based on the terrane characteristics (These include: Generalized Source Rock, Province, Tectonic Setting, and Scale). A brief discussion of these terms is provided below. These search fields are inherently simplified and may not adequately explain the character, especially of diverse terranes.

\section{Generalized Source Rock}

Five primary rock categories (sedimentary, volcanic, intrusive, metamorphic, and basement) are used in conjunction with five secondary rock categories (silicic, mafic, ultramafic, carbonate, and siliceous) to provide thirteen categories (table 3) to describe the generalized source rock. Note that in some cases, the choice between terms is arbitrary. For example, basement and metamorphic rock categories overlap, and in many cases are interchangable. Here, 'Metamorphic' is generally used in the Coast Ranges and Klamath Mountains, and 'Basement' in the Great Basin and Colorado Plateaus. In areas where several different source rocks may be present, multiple rock type terms have been used. 'Basement' is generally used throughout this report to refer loosely to dense, crystalline, and usually Precambrian rocks. This is in contrast to its use in the term 'basement gravity' (e.g., fig. 2), which considers basement as pre-Cenozoic rocks assumed to be dense, crystalline rocks of many types. Note that in places where source 
rocks are entirely covered, the inferred source rock type is generally inferred from the gravity and magnetic character of the terrane, and may not be unique. For example, a gravity and magnetic low that could be inferred as due to silicic basement might instead be due to depressed basement that has no lateral variation in basement composition.

Table 3. Generalized source rock categories

\begin{tabular}{|c|c|c|}
\hline Primary & Secondary & Usage \\
\hline \multirow{2}{*}{ sedimentary } & carbonate & $\begin{array}{l}\text { Generally used to refer to dense sedimentary rocks (e.g. limestone, } \\
\text { dolomite). }\end{array}$ \\
\hline & siliceous & $\begin{array}{l}\text { Generally used to refer to non-carbonate sedimentary rocks (e.g. sandstone, } \\
\text { siltstone, argillites, cherts). }\end{array}$ \\
\hline \multirow{2}{*}{ volcanic } & silicic & $\begin{array}{l}\text { Generally used for non-magnetic volcanic rocks. An exception to this is in } \\
\text { areas of silicic tuff. Generally includes intermediate composition rocks (e.g. } \\
\text { andesites). }\end{array}$ \\
\hline & mafic & Generally used for magnetic volcanic rocks. \\
\hline \multirow{3}{*}{ intrusive } & silicic & $\begin{array}{l}\text { Generally used for low density or non-magnetic intrusive rocks. Generally } \\
\text { includes intermediate composition rocks (e.g. dacites). }\end{array}$ \\
\hline & mafic & $\begin{array}{l}\text { Generally used for dense or magnetic intrusive rocks. Note that some } \\
\text { granitic rocks can be dense. }\end{array}$ \\
\hline & ultramafic & Generally used for dense or magnetic intrusive rocks. \\
\hline \multirow{3}{*}{ metamorphic } & silicic & $\begin{array}{l}\text { Generally used for low density or non-magnetic (e.g. quartzose) } \\
\text { metamorphic rocks. }\end{array}$ \\
\hline & mafic & Generally used for dense and magnetic metamorphic rocks. \\
\hline & ultramafic & Generally used for dense and magnetic metamorphic rocks. \\
\hline basement & silicic & Generally used for relatively low density or non-magnetic basement rocks. \\
\hline
\end{tabular}




\begin{tabular}{|c|c|l|} 
& mafic & Generally used for very dense and magnetic basement rocks. \\
\cline { 2 - 3 } & carbonate & Generally used for dense and non-magnetic basement rocks. \\
\hline
\end{tabular}

\section{Tectonic Setting}

The 'Tectonic Setting' category describes the tectonic setting associated with the development of the geophysical feature. In cases where a terrane formed from several geologic events or during multiple stages, or straddled different tectonic settings, multiple categories of 'Tectonic Setting' were used. As a result, both ancient and recent settings may be listed. Nonetheless, the most representative setting controlling the character of the feature is given (e.g., the 'Tectonic Setting' of Salinian granites in the Coast Ranges is given as both 'Batholith' and 'Transcurrent' because the shape of the block subsequent to the rocks having formed as a batholith was modified by transcurent strike-slip motion). In cases where multiple terms are given, the setting considered primarily responsible for the feature is listed first. When there is doubt as to the origin of the source, the present tectonic setting is given (e.g. 'Extension" applies to sources within the Great Basin with no known tectonic origin). Table 4 lists 'Tectonic Setting' categories that include eleven terms (extension, transcurrent, compression, stable crust, subduction, accretion, uplift, depression, hotspot, batholith, continental margin). The use of 'Subduction' to describe the tectonic setting of terranes within the Great Basin applies to Mesozoic intrusive rocks mainly in the western Great Basin (Walker Lane Belt) and Oligocene to middle Miocene volcanism that occurred throughout the Great Basin due to shallowing and subsequent steepening of the subducting Farallon slab.

Table 4: Tectonic setting categories

\begin{tabular}{|c|l|}
\hline Tectonic setting & \multicolumn{1}{|c|}{ Usage } \\
\hline extension & $\begin{array}{l}\text { Generally applies to entire Great Basin with Basin and Range type extension and to even } \\
\text { greater extended terranes. Also used to describe back-arc spreading as seen in the Oregon } \\
\text { highlands. }\end{array}$ \\
\hline
\end{tabular}




\begin{tabular}{|c|l|}
\hline transcurrent & $\begin{array}{l}\text { Refers to areas subjected to significant strike-slip deformation. Generally applied to right- } \\
\text { lateral displacements in Coast Ranges and the Walker Lane. }\end{array}$ \\
\hline compression & $\begin{array}{l}\text { Used in the region east of the Idaho Batholith and north of the Snake River Plain -- an area in } \\
\text { the Rocky Mountains Foreland Thrust Belt. }\end{array}$ \\
\hline stable crust & $\begin{array}{l}\text { Largely applies to the Colorado Plateaus region and refers to areas of weakly deformed flay- } \\
\text { lying Mesozoic and Paleozoic rocks. }\end{array}$ \\
\hline subduction & $\begin{array}{l}\text { Applies to major batholiths and to extensive magmatism in the Great Basin thought to relate } \\
\text { to shallowing of the subducting Farallon Plate in the later part of the Cenozoic. }\end{array}$ \\
\hline accretion & $\begin{array}{l}\text { Used extensively in California in the Coast Ranges, Klamath Mountains, and extending to } \\
\text { the western Sierra Nevada. These areas are often also associated with transcurrent tectonic } \\
\text { setting. }\end{array}$ \\
\hline uplift & $\begin{array}{l}\text { Used for blocks of basement uplifted on high-angle faults or on flextures. Areas include core } \\
\text { complexes and the Colorado Plateaus. }\end{array}$ \\
\hline depression & $\begin{array}{l}\text { Used largely for isolated basins in the California Coast Ranges. Also associated with areas of } \\
\text { pull-apart tectonics. Can also apply to depressed basement. }\end{array}$ \\
\hline hotspot & Used to refer to magmatism and fracturing associated with the ancestral Yellowstone hotspot. \\
\hline & $\begin{array}{l}\text { Used to refer to large granitoid intrusions associated with the Sierra Nevada, Salinian Block, } \\
\text { and Idaho Batholiths and to fragments of these such as may occur eastern California and } \\
\text { west-central Nevada. }\end{array}$ \\
\hline batholith & $\begin{array}{l}\text { Refers to Paleozoic and Mesozoic continental margin in the Great Basin. Defined esentially } \\
\text { by the edge of the continental shelf. }\end{array}$ \\
\hline \multirow{2}{*}{ continental margin }
\end{tabular}

\section{Provinces}

Geophysical terranes within twelve physiographic provinces, that include the Great Basin and parts of its surrounding provinces (Cascade Mtns., Coast Ranges, Colorado Plateaus, Columbia Plateaus, Great Valley, Klamath Mtns., Middle Rocky Mtns., Mojave Desert, Northern Rocky Mtns., Sierra Nevada, Snake River Plain) are described in this report (figs. 2 and 3, table 1). Figure 1 shows the extent of these provinces (note that the province boundaries used here largely follow those of Fenneman and Johnson (1946), and table 2 gives their physiographic, geologic and geophysical descriptions.

In places, province boundaries are poorly defined, for example, at the boundary between the Great Basin and Columbia Plateaus Provinces. This boundary, which is not well expressed physiographically, represents one of the few differences between the province map used here and that of others (e.g. Fenneman and Johnson, 1946). Terms used under the 'Generalized Tectonic Setting' column refer to the same terms used in Table 4 and described above. 


\section{Scale of Geophysical Features}

At the broadest scales, geophysical terranes can reflect major deep-seated crustal discontinuities such as transform, accommodation, or shear zones, ancient continental margins, failed rifts, accretionary belts, or magmatic arcs. At local scales, they can reflect, for example, individual faults or intrusive bodies. Below is a description and definition of terrane scales that appear in table 1.

\section{Regional-scale Geophysical Provinces}

Regional scale terranes are considered to constitute very large regions, extending from hundreds to thousands of kilometers, that may consist of an assemblage of smaller-scale features that share a common character in contrast to surrounding regions (e.g. the terrane may define a zone of consistent geophysical fabric). A regional-scale feature may reflect a region of common tectonic or magmatic history and it may be bound by deep crustal to subcrustal faults. Some examples include volcanic plateaus, broad shear zones, and broad and coherent crustal blocks bound by deep crustal faults. Identification of regional-scale features is aided by standard and longwavelength geophysical maps, fabric analysis, contrasts of dominant frequencies, and contrasts in mean gravity and magnetic values.

A description of the regional geophysical expression of the Great Basin and each of its surrounding provinces is given in table 2 . The broadest expression in regional gravity and magnetic maps of the study area are reflected in figure 4, which show outlines that roughly mimic the shape and extent of the entire Great Basin. These largest of terranes appear in table 1 as the first two entries.

The Great Basin boundary has relatively sharply defined geophysical boundaries, though these, in places, crosscut the physiographically and geologically defined boundaries of the region. To the north it contrasts with the high basement gravity and magnetic character of the Snake River Plain, although the gravity high over the northern Great Basin merges with that of the Snake River Plain. To the west, the high frequency magnetic and moderate gravity highs of the western 
Great Basin abut the prominent northwest-trending magnetic high and gravity low of the eastern Sierra Nevada Province. At its east edge, the Great Basin is rimmed by the Colorado Plateaus. Though its contact is not particularly clear in the basement gravity, the generally low magnetic fields over the Great Basin contrast sharply with the high magnetic terrain of the Colorado Plateaus. More ambiguous occur to the northeast, northwest, and south where the Great Basin meets with Middle Rocky Mountains, Columbia Plateaus, and Mojave Desert Provinces, respectively.

\section{Intermediate-Scale Geophysical Features}

Intermediate-scale terranes constitute large coherent anomalies (e.g., crustal rifts or sutures, structural basins or ranges, or batholiths) that extend on the order of tens to hundreds of kilometers. Identification of these regional-scale features is aided by standard geophysical maps, long- and short-wavelength maps, and AMHG maps.

\section{Local-Scale Geophysical Features}

Local-scale features (reflecting, for example, individual plutons, faults, or calderas) reflect anomalies arising from discrete source bodies that reside in the shallow to mid-crust, and extend over several tens of kilometers. Identification of local-scale features is aided by standard geophysical, long- and short-wavelength, and AMHG maps. Although a few local-scale anomalies are described in this report, a detailed assessment of local-scale anomalies is beyond the scope of this study.

\section{DISCUSSION}

This work provides an introduction to geophysical terranes of the Great Basin and surrounding regions, with interpretations of their underlying causes. The aim of this work is to relate geophysical terranes to their geologic domain counterparts, to resolve the nature of transitions 
between terranes, and to understand their origin, and their relation to basement structures and composition.

The study spans local-scale (e.g., individual plutons, faults, or calderas), anomalies arising from discrete source bodies that reside in the shallow to mid-crust, and extend over an order of several tens of kilometers to regional-scale features (very large regions, extending from hundreds to thousands of kilometers). Assessment of geophysical terranes was aided by a variety of filtering and derivative methods, and took into account frequency, amplitude, fabric, and gradients of terranes anomalies.

Geophysical maps and data tables of this report are provided in a variety of formats (tab delimited text, Microsoft Excel, Microsoft Word, PDF, and ArcGIS) for readers to review and download. The PDF formatted product contains useful links that allow the user to easily move between features on the maps and their entries in the tables.

\section{ACKNOWLEGMENTS}

We thank George Billingsley, Richard Blakely, Mark Gettings, Bob Jachens, David John, Victor Mossotti, David Piper, Ted Theodore, and Allan Wallace for helpful reviews and discussions, and Barry Moring for assisting in technical preparation of this manuscript.

\section{REFERENCES CITED}

Baranov, V., 1957, A new method for interpretation of aeromagnetic maps: pseudo-gravimetric anomalies: Geophysics, v. 22, p. 359-383.

Best, M.G., Christiansen, E.H., and Blank, R.H., Jr., 1989a, Oligocene caldera complex and calc-

alkaline tuffs and lavas of the Indian Peak volcanic field, Nevada and Utah: Geological Society of America Bulletin, v. 101, p. 1076-1090. 
Best, M.G., Christiansen, E.H., Deino, A.L., Gromme, C.S., McKee, E.H., and Noble, D.C., 1989b, Excursion 3A - Eocene through Miocene volcanism in the Great Basin of the western United States: New Mexico Bureau of Mines and Mineral Resources Memoir 47, p. 91-133.

Best, M.G., McKee, E.H., and Damon, P.E., 1980, Space-time composition patterns of Late Cenozoic mafic volcanism, southwestern Utah and adjoining areas: American Journal of Science, v. 280, p. 1035-1050.

Blackwell, D.D., 1978, Heat flow and energy loss in the Western United States, in Smith, R.B., and Eaton, G.P., eds., Cenozoic tectonics and regional geophysics of the Western Cordillera: Geological Society of America Memoir 152, p.175-208.

Blakely, R.J., 1988, Curie temperature isotherm analysis and tectonic implications of aeromagnetic data from Nevada: Journal of Geophysical Research, v. 93, p. 11,817-11,832.

Blakely, R.J., 1995, Potential theory in gravity and magnetic applications: New York, Cambridge University Press, $441 \mathrm{p}$.

Blakely, R.H., and Jachens, R.C., 1991, Regional study of mineral resources in Nevada: Insights from three-dimensional analysis of gravity and magnetic anomalies: Geological Society of America Bulletin, v. 103, p. 795-803.

Blakely, R.H., Jachens, R.C., Simpson, R.W., and Couch, R.W., 1985, Tectonic setting of the southern Cascade Range as interpreted from its magnetic and gravity fields, Geological Society of America Bulletin, v. 96, p. 43-48.

Blakely, R.J, Schruben, P.G, and Moring, B.C., 1996, Shallow magnetic lithologies as interpreted from low-altitude aeromagnetic data, in Singer, D.A., An analysis of Nevada's metalbearing mineral resources: Nevada Bureau of Mines and Geology Open-File Report 96-2, p. 3.13.9 .

Blakely, R.J., and Simpson, R.W., 1986, Approximating edges of source bodies from gravity or magnetic data: Geophysics, v. 51, p. 1494-1498.

Blank, H.R., Jr., 1988, Basement structure in the Las Vegas region from potential-field data: Geological Society of America, Abstracts with Programs, v. 20, no. 3, p. 144. 
Blank, H.R., Butler, W.C., and Saltus, R.W., 1998, Neogene uplift and radial collaps of the Colorado Plateau - regional implications of gravity and aeromagnetic data: U.S. Geological Survey Bulletin 2158, p. 9-32.

Bott, M.H.P., and Smithson, S.B., 1967, Gravity investigations of subsurface shape and mass distributions of granite batholiths: Geological Society of America Bulletin, v. 78, p. 859-878.

Burnett, J.L., and Jennings, C.W., 1982, Bouguer gravity map of California, Chico Sheet: California Division of Mines and Geology Map, scale 1:250,000.

Byerly, P.E., 1966, Interpretations of gravity data from the central Coast Ranges and San Joaquin valley: California Geological Society of America Bulletin, v. 77, p. 83-94.

Byers, F.M., Jr., Carr, W.J., Orkild, P.P., Quinlivan, W.D., and Sargent, K.A., 1976, Volcanic suites and related caldrons of Timber Mountain-Oasis Valley caldera complex, southern Nevada: U.S. Geological Survey Professional Paper 919, 70 p.

Cady, J.W., 1975, Magnetic and gravity anomalies in the Great Valley and western Sierra Nevada metamorphic belt, California: Geological Society of America Special Paper 168, 56p.

Case, J.E., and Joesting, H.R., 1972, Regional geophysical investigations in the central Colorado Plateau: U. S. Geological Survey Professional Paper 736, 31p.

Chapman, R.H., and Griscom, A., 1980, Coast Ranges, in Oliver, H.W., ed., Interpretation of the gravity map of California and its continental margin: California Division of Mines and Geology Bulletin 205, p. 24-27.

Chapman, R.H., Healey, D.L., and Toxel, B.W., 1973, Bouguer gravity map of California, Death Valley sheet: California Division of Mines and Geology Map, scale 1:250,000.

Chen, J.H., and Moore, H, G, 1982, Uranium-lead isotopic ages from the Sierra Nevada Batholith, California: Journal of Geophysical Research, v. 87, no. 129-133.

Cordell, L., and McCafferty, A.E., 1989, A terracing operator for physical property mapping with potential field data: Geophysics, v. 54, p. 621-634. 
Craig, S.D., ed., 1992, Walker Lane Symposium: structure, Tectonics and mineralization of the Walker Lane: Geological Society of Nevada, Proceeding, v. 14, April 24, 65p.

Dallmeyer, R.D., Snook, A.W., and McKee, E.H., 1986, Mesozoic-Cenozoic tectonothermal evolution of the Ruby Mountains, east Humboldt Range, Nevada: a Cordilleran metamorphic core complex: Tectonics, v. 5, p. 931-954.

Davis, G.A., and Burchfiel, B.C., 1973, Garlock Fault: An Intracontinental Transform Structure, Southern California: Geological Society of America Bulletin, v. 84, p. 1407-1422.

Dilek, Y., and Moores, E.M., 1995, Geology of the Humboldt igneous complex, Nevada, and tectonic implications for the Jurassic magmatism in the Cordilleran Orogen: Geological Society of America Special Paper 299, p. 229-248.

Dobrin, M.B., and Savit, C.H., 1988, Introduction to Geophysical Prospecting (4th ed.): New York, McGraw-Hill, 867p.

Eaton, G.P., Wahl, R.R., Prostka, H.J., Mabey, D.R., and Kleinkopf, M.D., 1978, Regional gravity and tectonic patterns: Their relation to late Cenozoic epeirogeny and lateral spreading in the western Cordillera, in Smith, R.B., and Eaton, G.P., eds., Cenozoic tectonics and regional geophysics of the western Cordillera: Geological Society of America Memoir 152, p. 51-91.

Eckel, E.B., ed., 1968, Nevada Test Site: Geological Society of America Memoir 110, 290 p.

Fenneman, N.M., 1931, Physiography of western United States: New York and London, McGraw Hill Co, 534p.

Fenneman, N.M., and Johnson, D.W., 1946, Map of physical divisions of the United States, Prepared in cooperation with the Physiographic Committee of the Geological Survey, scale $1: 7,000,000$.

Glen, J.M.G., and Ponce, D.A., 2002, Large-scale fractures related to inception of the Yellowstone hotspot: Geology, v. 30, p. 647-650. 
Glen, J.M.G., Hildenbrand, T.G., and Ludington, S., 2000, Insights on the basement of the Great Basin from an integrated geophysical, geologic and geochemical study: Geological Society of Amererica, Abstracts with programs, v. 32, p. A503.

Grant, F.S., 1985a, Aeromagnetics, geology and ore environments; II, Magnetite and ore environments: Geoexploration, v. 23, p. 335-362.

Grant, F.S., 1985b, Aeromagnetics, geology and ore environments; I, Magnetite in igneous, sedimentary and metamorphic rocks; an overview: Geoexploration, v. 23, p. 303-333.

Grauch, V.J.S., 1996, Magnetically interpreted, granitoid plutonic bodies in Nevada, in Singer, D.A., ed., An analysis of Nevada's metal-bearing mineral resources: Nevada Bureau of Mines and Geology Open-File Report 96-2, p. 7.1-7.16.

Grauch, V.J.S., and Cordell, L., 1987, Limitations of determining density or magnetic boundaries from the horizontal gradient of gravity or pseudogravity data: Geophysics, v. 52, p. 118-121.

Grauch, V.J.S., Jachens, R.C., and Blakely, R.J., 1995, Evidence for a basement feature related to the Cortez disseminated gold trend and implications for regional exploration in Nevada: Economic Geology, v. 90, p. 203-207.

Grauch, V.J.S., Sawyer, D.A., Fridrich, C.J., and Hudson, M.R., 1999, Geophysical framework of the southwestern Nevada volcanic field and hydrogeologic implications: U.S. Geological Survey Professional Paper 1608, 39p.

Griscom, A., 1966, Magnetic data and regional structure in northern California, in Geology of northern California: California Division of Mines and Geology Bulletin 190, p. 407-417.

Griscom, A., 1973, Bouguer gravity map of California, Redding Sheet: California Division of Mines and Geology Map, scale 1:250,000.

Griscom, A., 1980a, Cascade Range and Modoc Plateau, in Oliver, H.W., ed., Interpretation of the gravity map of California and its continental margin, California Division of Mines and Geology, Bulletin 205, p. 36-38. 
Griscom, A., 1980b, Klamath Mountains Province, in Oliver, H.W., ed., Interpretation of the gravity map of California and its continental margin: California Division of Mines and Geology, Bulletin 205, p. 34-36.

Griscom, A., and Jachens, R.C., 1990, Tectonic implications of gravity and magnetic models along east-west seismic profiles across the Great Valley near Coalinga, in Rymer, M.J., and Ellsworth, W.L., eds., The Coalinga, California earthquake of May 2, 1983: U.S. Geological Survey Professional Paper 1487, p. 69-78.

Griscom, A., and Oliver, H.W., 1980, Isostatic gravity highs along the west side of the Sierra Nevada and the Peninsular Ranges batholiths, California: Eos, Transactions of the American Geophysical Union, v. 61, p. 1126.

Gunn, P.J., and Dentith, M.C., 1997, Magnetic response associated with mineral deposits, in Gunn, P.J., ed., Airborne magnetic and radiometric surveys: AGSO Journal of Australian Geology and Geophysics, v. 17, p. 145-158.

Hanna, W.F., Burch, S.H., and Dibblee, T.W., Jr., 1972, Gravity, magnetics, and geology of the San Andreas Fault area near Cholame, California: U. S. Geological Survey Professional Paper 646-C, p. C1-C29.

Healey, D.L., 1970, Bouguer gravity map of California, Kingman Sheet: California Division of Mines and Geology Map, scale 1:250,000.

Henry, C.D., and Ressel, M.W., 2000, Interpretation of Eocene magmatism, extension, and Carlin-type gold deposits in northeastern Nevada, in Lageson, D.R., Peters, S.G., and Lahren, M.M., eds., Great Basin and Sierra Nevada: Geological Society of America Field Guide 2, p. 165-187.

Hildenbrand, T.G., Berger, B., Jachens, R.C., and Ludington, S., 2000, Regional crustal structures and their relationship to the distribution of ore deposits in the western United States, based on magnetic and gravity data: Economic Geology, v. 95, p. 1583-1603. 
Hinze, W.J., and Zietz, I., 1985, The composite magnetic-anomaly map of the conterminous United States, in Hinze, W.J., ed., The utility of regional gravity and magnetic anomaly maps: 52nd annual international meeting of the Society of Exploration Monograph, p. 1-24.

Hutchinson, R.W., and Albers, H.P., 1992, Metallogenic evolution of the Cordilleran region of the Western United States, in Burchfiel, B C, Lipman, P.W., and Zoback, M.L., eds., The geology of North America, The Cordilleran orogen: Contermicous U.S.: Boulder, Colorado, Geological Society of America, v. G-3, p. 629-652.

Jachens, R.C., and Griscom, A., 1985, An isostatic gravity map of California: a residual map for interpretation of anomalies from intracrustal sources, in Hinze, W.J., ed., The utility of regional gravity and magnetic anomaly maps: Tulsa, Oklahoma, Society of Exploration Geophysicists, p. 347-360.

Jachens, R.C., and Griscom, A., in press, Geologic and Geophysical setting of the 1989 Loma Prieta earthquake, California, inferred from magnetic and gravity anomalies: U.S. Geological Survey Professional Paper.

Jachens, R.C., and Griscom, A., and Roberts, C.W., 1995, Regional extent of Great Valley basement west of the Great Valley, California: implications for extensive tectonic wedging in the California Coast Ranges: Journal of Geophysical Research, v. 100, p. 12769-12790.

Jachens, R.C., and Moring, B.C., 1990, Maps of the thickness of Cenozoic deposits and the isostatic residual gravity over basement for Nevada: U.S. Geological Survey Open-File Report $90-404,15 p$.

Jachens, R.C., Simpson, R.W., Blakely, R.J., and Saltus, R.W., 1989, Isostatic residual gravity and crustal geology of the United States, in Pakiser, L.C., and Mooney, W.D., eds., Geophysical framework of the continental United States: Geological Society of America Memoir 172, p. 405424.

Jachens, R.C., Wentworth, C.M., and McLaughlin, R.J., 1998, Pre-San Andreas location of the Gualala block inferred from Magnetic and gravity anomalies, in Geology and tectonics of the Gualala block, northern California: SEPM Pacific Section Book 84, p. 27-64. 
John, D.A., Wallace, A.R., Ponce, D.A., Fleck, R.B., and Conrad, J.E., 2000, New perspectives on the geology and origin of the northern Nevada Rift, in Cluer, J.K., Price, J.G., Struhsacker, E.M., Hardyman, R.F., and Morris, C.L., eds., Geology and Ore Deposits 2000: The Great Basin and Beyond: Geological Society of Nevada Symposium Proceedings, May 15-18, p. 127-154.

Kane, M.F., and Godson, R.H., 1985, Features of a pair of long-wavelength $(>250 \mathrm{~km})$ and short-wavelength $(<250 \mathrm{~km})$ Bouguer gravity maps of the United States, in Hinze, W.J., ed., The utility of regional gravity and magnetic anomaly maps: 52nd annual international meeting of the Society of Exploration Monograph, p. 46-61.

Karlstrom, K.E., Bowring, S.A., Chamberlain, K R; Dueker, K.G., Eshete, T., Erslev, E.A., Farmer, G.L., Heizler, M., Humphreys, E.D., Johnson, R.A., Keller, G.R., Kelley, S.A., Levander, A., Magnani, M.B., Matzel, J.P., McCoy, A.M., Miller, K.C., Morozova, E.A., Pazzaglia, F.J., Prodehl, C., Rumpel, H.M., Shaw, C.A., Sheehan, A.F., Shoshitaishvili, E., Smithson, S.B., Snelson, C.M., Stevens, L.M., Tyson, A.R., and Williams, M.L., 2002, Structure and evolution of the lithosphere beneath the Rocky Mountains; initial results from the CD-ROM experiment, Continental Dynamics of the Rocky Mountains Working Group, United States: GSA Today, v. 12, p. 4-10.

Kleinhampl, F.H., and Ziony, J.I., 1984, Mineral resources of northern Nye County, Nevada: Nevada Bureau of Mines and Geology Bulletin 99B, 243p.

Kleinhampl, F.J., Davis, W.E., Silberman, M.L., Chesterman, C.W., Chapman, R.H., and Gray, C.H., Jr., 1975, Aeromagnetic and limited gravity studies and generalized geology of the Bodie Hills region, Nevada and California: U. S. Geological Survey Bulletin 1384, 38p.

Kuntz, M.A., Champion, D.E., Lefebvre, R.H., and Covington, H.R., 1988, Geologic map of the Craters of the Moon, Kings Bowl, and Wapi lava fields, and the Great Rift volcanic rift zone, south-central Idaho: U. S. Geological Survey Miscellaneous Investigations Series I-1632.

Kuntz, M.A., Covington, H.R., and Schorr, L.J., 1992, An overview of basaltic volcanism of the eastern Snake River plain, Idaho: Geological Society of America Memoir 179, p. 227-267.

Lachenbruch, A.H., and Sass, J.H., 1978, Heat flow in the United States and the thermal regime of the crust: U. S. Geological Survey Professional Paper 1100, p. 200-201. 
LaFehr, T.R., 1965, Gravity, isostasy, and crustal structure in the southern Cascade Range: Journal of Geophysical Research, v. 70, p. 5581-5597.

LaFehr, T.R., 1966, Gravity in the eastern Klamath Mountains, California: Geological Society of America Bulletin, v. 77, p. 1177-1190.

Langenheim, V.E., Glen, J.M.G., Jachens, R.C., Dixon, G.L., Katzer, T.C., and Morin, R.L., 2000, Geophysical constraints on the Virgin River Depression, Nevada, Utah, and Arizona: U.S. Geological Survey Open-File Report 00-0407.

Mabey, D.R., 1961, Regional magnetic and gravity anomalies in the Darwin area, California: U. S. Geological Survey Professional Paper 0424-C, p. C276-C279.

Mabey, D.R., 1976, Interpretation of a gravity profile across the western Snake River Plain, Idaho: Geology, v. 4, p. 53-55.

Mabey, D.R., 1982, Geophysics and tectonics of the Snake River plain, Idaho, in Bonnichsen, B., and Breckenridge, R.M., eds., Cenozoic geology of Idaho: Idaho Bureau of Mines and Geology Bulletin 26, p. 139-153.

Mabey, D.R., 1986, Gravity and magnetic anomaly maps of the Tenmile West Roadless Area, Boise and Elmore counties, Idaho: U. S. Geological Survey,Miscellaneous Field Studies Map MF-1500-D, 1 sheet.

Mabey, D.R., Zietz, I., Eaton, G.P., and Kleinkopf, M.D., 1978, Regional magnetic patterns in part of the Cordillera in the western United States, in Smith, R.B., and Eaton, G.P., eds., Cenozoic tectonics and regional geophysics of the western Cordillera: Geological Society of America Memoir 152, p. 93-106.

Mankinen, E.A., Hildenbrand, T.G., Dixon, G.L., McKee, E.H., Fridrich, C.J., and Laczniak, R.J., 1999, Gravity and magnetic study of the Pahute Mesa and Oasis Valley region, Nye County, Nevada: U.S. Geological Survey Open-File Report 99-303, 35p.

McKee, E.H., 1968, Geology of the Magurder Mountain area, Nevada-California: U.S. Geological Survey Bulletin 1251-H, p. 1-40. 
McKee, E.H., Duffield, W.A., and Stern, R.J., 1983, Late Miocene and early Pliocene basaltic rocks and their implications for crustal structures, northeastern California and south-central Oregon, Geological Society of America Bulletin, v. 94, p. 292-304.

McKee, E.H., Hildenbrand, T.G., Anderson, M.L., Rowley, P.D., and Sawyer, D.A., 1999, The Silent Canyon caldera complex - a three-dimensional model based on drill-hole stratigraphy and gravity inversion: U.S., Geological Survey Open-File Report 99-555, 38p.

McKee, E.H., Jachens, R.C., and Blakely, R.J., 1990, Major crustal differences between the northwestern part of the Great Basin and other parts of the province, in Cuffney, B., ed., Geology and ore deposits of the Great Basin: Geological Society of Nevada and U.S. Geological Survey Program with abstracts, p. 100.

McKee, E.H., and Nelson, C.A., 1967, Geologic map of the Soldier Pass Quadrangle, California and Nevada: U. S. Geological Survey Geologic Quadrangle Map Report GQ-0654.

McKee, E.H., Phelps, G.A., and Mankinen, E.A., 2001, The Silent Canyon caldera - a three dimensional model as part of a Pahute Mesa - Oasis Valley hydrogeologic model: U.S. Geological Survey Open-File Report 01-297.

Noble, D.C., and McKee, E.H., 1972, Description and K-Ar ages of volcanic units of the Caliente volcanic field, Lincoln County, Nevada, and Washington County, Utah: Isochron/West, v. 5, p. 17-24.

Oldow, J.S., 1984, Evolution of a late Mesozoic back-arc fold and thrust belt, northwestern Great Basin, U.S.A., Tectonophysics, 102, 245-274.

Oliver, H.W., 1977, Gravity and magnetic investigations of the Sierra Nevada Batholith, California: Geological Society of America Bulletin, v. 88, p. 445-461.

Oliver, H.W., 1980, Sierra Nevada, in Oliver, H.W., ed., Interpretation of the gravity map of California and its continental margin: California Division of Mines and Geology Bulletin 205, p. 30-32. 
Oliver, H.W., and Griscom, A., 1980, Great Valley, in Oliver, H.W., ed., Interpretation of the gravity map of California and its continental margin: California Division of Mines and Geology, Bulletin 205, p. 27-29.

Oliver, H.W., and Mabey, D.R., 1963, Anomalous gravity field in east-central California, Geological Society of America Bulletin, v. 74, p. 1293-1298.

Oliver, H.W., Moore, J.G., and Sikora, R.F., 1992, Internal structure and vertical extent of the Sierra Nevada Batholith, California, from specific-gravity and gravity data, in Guangzhi, T., Keqin, Xu. And Yuzhou, Q., eds., Petrogenesis and mineralization of granitoids, Proceedings of 1987 Guangzhou Internationl Symposium: Beijing, China, Science Press.

Oliver, H.W., Ponce, D.A., and Hunter, W. Clay, 1995, Major results of geophysical investigations at Yucca Mountain and vicinity, southern Nevada: U.S. Geological Survey OpenFile Report 95-74, 184 p.

Page, B.M., 1965, Preliminary geologic map of a part of the Stillwater Range, Churchill County, Nevada: Nevada Bureau of Mines and Geology Map 28, scale 1: 125,000.

Pierce, K.L., Morgan, L.A., and Saltus, R.W., 2000, Yellowstone plume head: postulated tectonic relations to the Vancouver slab, continental boundaries and climate: U.S. Geological Survey Open-File Report 00-498, 39p.

Ponce, D.A., and Glen, J.M.G., 2002, Relationship of epithermal gold deposits to large-scale fractures in northern Nevada: Economic Geology, v. 97, p. 3-9.

Reford, M.S., 1980, Magnetic method, in Domenico, S.N., and Silverman, D., eds., Fifty years of progress; a review; 1930-1980: Geophysics, v. 45, p. 1640-1658.

Roberts, C.W., and Jachens, R.C., 1993, Isostatic residual gravity map of the San Francisco Bay area, California: U.S. Geological Survey Geophysical Investigations Map GP-1006.

Rowan, L.C., and Wetlaufer, P.H., 1973, Structural geologic analysis of Nevada using ERTS-1 images: a preliminary report: NASA Special Publication 327, p. 413-423. 
Rytuba, J.J., and McKee, E.H., 1984, Perakaline ash-flow tuffs and calderas of the McDermitt volcanic field, southeast Oregon and north-central Nevada: Journal of Geophysical Research, v. 89, p. 9616-8628.

Saltus, R.W., Thompson, G.A., 1995, Why is it downhill from Tonopah to Las Vegas? A case for mantle plume support of the high northern Basin and Range: Tectonics, v. 14, p. 1235-1244.

Simpson, R.W., and Jachens, R.C., 1989, Gravity methods in regional studies, in Pakiser, L.C., Mooney, W.D., eds., Geophysical framework of the continental United States, Geological Society of America Memoir 172, p. 35-44.

Simpson, R.W., Jachens, R.C., Blakely, R.J., and Saltus, R.W., 1986, A new isostatic residual gravity map of the conterminous United States with a discussion on the significance of isostatic residual anomalies: Journal of Geophysical Research, v. 91, p. 8348-8372.

Sims, P.K., Bankey, V., and Finn, C.A., 2001, Preliminary Precambrian basement map of Colorado -- A geologic interpretation of the aeromagnetic anomaly map: U.S. Geological Survey Open-File Report 01-364.

Smith, R.B., 1978, Seismicity, crustal structure, and intraplate tectonics of the interior of the western Cordillera, in Smith, R.B., and Eaton, G.P., eds., Cenozoic tectonics and regional geophysics of the Western Cordillera: Geological Society of America Memoir 152, p.111-144.

Sparlin, M.A., Braile, L.W., and Smith, R.B., 1982, Crustal structure of the eastern Snake River plain determined from ray trace modeling of seismic refraction data: Journal of Geophysical Research, v. 87, p. 2619-2633.

Speed, R.C., 1976, Geologic map of the Humboldt lopolith: Geological Society of America Map and Chart Series MC-14, scale 1:81,050.

Stewart, J.H., 1988, Tectonics of the Walker Lane belt, western Great Basin: Mesozoic and Cenozoic deformation in a zone of shear, in Ernst, W.G., ed., Metamorphism and crustal evolution of the western United States, Rubey volume VII: Englewood Cliffs, New Jersey, Prentice Hall, p. 683-713. 
Stewart, J.H., and Carlson, J.E., 1978, Geologic map of Nevada: Nevada Bureau of Mines and Geology Map, scale 1:500,000.

Stewart, J.H., and Crowell, J.C., 1992, Strike-slip tectonics in the Cordilleran region, Western United States, in Burchfiel, B.C., Lipman, P.W., and Zoback, M.L., The geology of North America, the Cordilleran Orogen; conterminous U.S.: Boulder, Colorady: Geological Society of America, v. G-3, p. 609-628.

Stewart, J.H., Moore, W.J., and Zietz, I., 1977, East-west patterns of Cenozoic igneous rocks, aeromagnetic anomalies, and mineral deposits, Nevada and Utah: Geological Society of America Bulletin, v. 88, p. 67-77.

Sumner, J.S., 1985, Crustal geology of Arizona as interpreted from magnetic, gravity, and geologic data, in Hinze, W.J., ed., The utility of regional gravity and magnetic anomaly maps: 52nd annual international meeting of the Society of Exploration Monograph, p. 164-180.

Suppe, J., 1979, Structural interpretation of the southern part of the northern Coast Ranges and Sacramento Valley, California: Geological Society of America Bulletin, v. 90, p. I327-I 330.

Thompson and McCarthy, 1990, A gravity constraint on the origin of highly extended terranes: Tectonophysics, v.174, p.197-206.

U. S. Geological Survey, 1972, Aeromagnetic map of the Vya and part of the McDermitt 1 degrees by 2 degrees quadrangles, Nevada, U.S. Geological Survey Open-File Report 72-0393.

U. S. Geological Survey, 1982a, Spectral radiometric and total-field magnetic survey of the McDermitt calderas, NV-OR: U. S. Geological Survey Open-File Report 82-0323-A, 48p.

U. S. Geological Survey, 1982b, Stacked magnetic and radiometric profiles of flight lines in the McDermitt calderas area, Nevada-Oregon: U.S. Geological Survey Open-File Report 82-0323-B, $96 \mathrm{p}$.

Vallier, T.L., and Brooks, H.C., eds., 1986, Geology of the Blue Mountains region of Oregon, Idaho and Washington: Geologic implications of Paleozoic and Mesozoic paleontology and biostratigraphy, Blue Mountains Province, Oregon and Idaho: U.S. Geological Survey Professional Paper 1435, 93p. 
Vallier, T.L., and Brooks, H.C., eds., 1994, Geology of the Blue Mountains region of Oregon, Idaho and Washington: Stratigraphy, physiography and mineral resources of the Blue Mountains region: U.S. Geological Survey Professional Paper 1439, 198p.

Vallier, T.L., and Brooks, H.C., eds., 1995, Geology of the Blue Mountains region of Oregon, Idaho and Washington: Petrology and tectonic evolution of pre-Tertiary rocks of the Blue Mountains region: U.S. Geological Survey Professional Paper 1438, 540p.

Vogel, T.A., Cambray, F.W., and Constenius, K.N., 2001, Origin and emplacement of igneous rocks in the central Wasatch Mountains, Utah: Rock Mountain Geology, v. 36, p. 119-162.

Wells, R.E., Weaver, C.S., and Blakely, R.J., 1998, Fore-arc migration in Cascadia and its neotectonic significance: Geology, v. 26, p. 759-762.

Woollard, G.P., 1972, Regional variations in gravity, in Robertson, E.C., ed., The Nature of the Solid Earth: New York, McGraw-Hill, p. 463-505.

Wyld, S.J., and Wright, J.E., 2001, New evidence for Cretaceous strike-slip faulting in the United States Cordillera and implications for terrane-displacement, deformation patterns, and plutonism: American Journal of Science, v. 301, p. 150-181.

Zietz, I., Bateman, P.C., Case, J.E., Crittenden, M.D., Jr., Griscom, A., King, E.R., Roberts, R.J., and Lorentzen, G.R., 1969, Aeromagnetic investigation of crustal structure for a strip across the western United States: Geological Society of America Bulletin, v. 80, p. 1703-1714.

Zoback, M.L., and Thompson, G.A., 1978, Basin and range rifting in northern Nevada; clues from a mid-Miocene rift and its subsequent offsets: Geology, v. 6, p. 11-116.

Zoback, M.L., and Zoback, M.D., 1980, Magnitude of deviatoric stresses in the Earth's crust and uppermost mantle: Journal of Geophysical Research, v. 85, p. 6113-6156.

Zoback, M.L., McKee, E.H., Blakely, R.J., and Thompson, G.A., 1994, The northern Nevada rift; regional tectono-magmatic relations and middle Miocene stress direction: Geological Society of America Bulletin, v. 106, p. 371-382. 


\section{TABLES}

Table 1. Geophysical features of the Great Basin and parts of surrounding provinces. Terranes appear in the table sorted in the order of: state, gravity number, and magnetic number. Refer to gravity and magnetic terrane maps (figs. 2 and 3, respectively). The table gives (from left to right): Scale of anomaly ( $\mathrm{R}=$ Regional, $\mathrm{I}=$ Intermediate, $\mathrm{L}=$ Local); Terrane ID (containing from left to right: 2 digits for the state, 1 ' $\mathrm{m}$ ' or ' $\mathrm{g}$ ' for magnetic or gravity anomaly, respectively; two digits for terrane number); Terrane definition (describes the geophysical characteristics that define the feature), Anomaly type ( $\mathrm{H}=$ high, $\mathrm{L}=$ low, or $\mathrm{B}=$ both high and low); Geologic Province (describes the geology associated with the geophysical terrane); Inferred source of anomaly; Generalized source rock (see Table 3 for description of terms); Tectonic setting (see table 4 for description of terms); Province (gives the associated physiographic province, see table 2);

References. Abbreviations include: BR, Basin and Range; CP, Colorado Plateau; CRP, Columbia River Plateaus; Cz, Cenozoic; GB, Great Basin; Mz, Mesozoic; NNR, Northern Nevada Rift; pC, Precambrian; Pz, Paleozoic; SRP, Snake River Plain. For further details, see text.

Table 2. Physiographic provinces and their geologic and geophysical character. Physiographic province boundaries and descriptions are modified from Fenneman and Johnson (1946).

\section{FIGURES}

Figure 1. A) Index map of the Great Basin showing digital shaded relief and outlines of the Great Basin and surrounding physiographic provinces. Physiographic province boundaries are modified from Fenneman and Johnson (1946); B) The Basin and Range extensional province that includes the Great Basin.

Figure 2. Basement gravity terrane map of the study area. Blue lines are edges of gravity sources determined by the maximum horizontal gravity method; each feature is uniquely labeled and described in Table 1. Yellow lines are linear gravity features, labeled uniquely and described in Table 1; red lines are unlabeled, undescribed, linear gravity features. White line 
shows boundary within which basement calculation was performed. Isostatic gravity is shown outside this boundary.

Figure 3. Magnetic potential terrane map of the study area. Blue lines are edges of magnetic sources determined by the maximum horizontal gravity method; each feature is uniquely labeled and described in Table 1. Yellow lines are linear magnetic features, labeled uniquely and described in Table 1; red lines are unlabeled, undescribed, linear magnetic features.

Figure 4. Regional isostatic gravity and pseudogravity field maps of the Great Basin and surrounding area. The white outline represents the Great Basin boundary in each map. The black outlines represent the regional gravity terrane and regional pseudogravity terrane described in Table 1 as G1 and M1, respectively. 

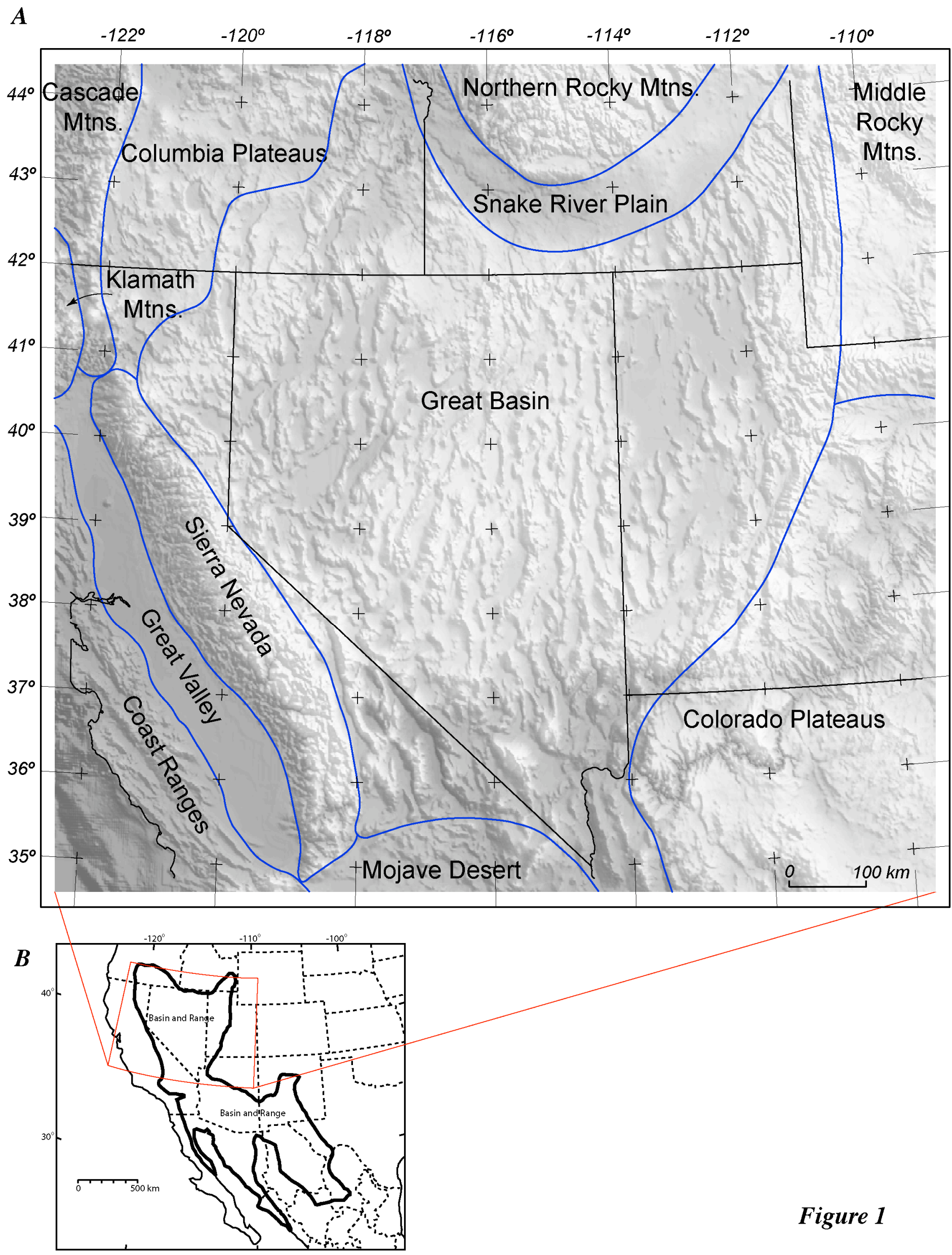

Figure 1 


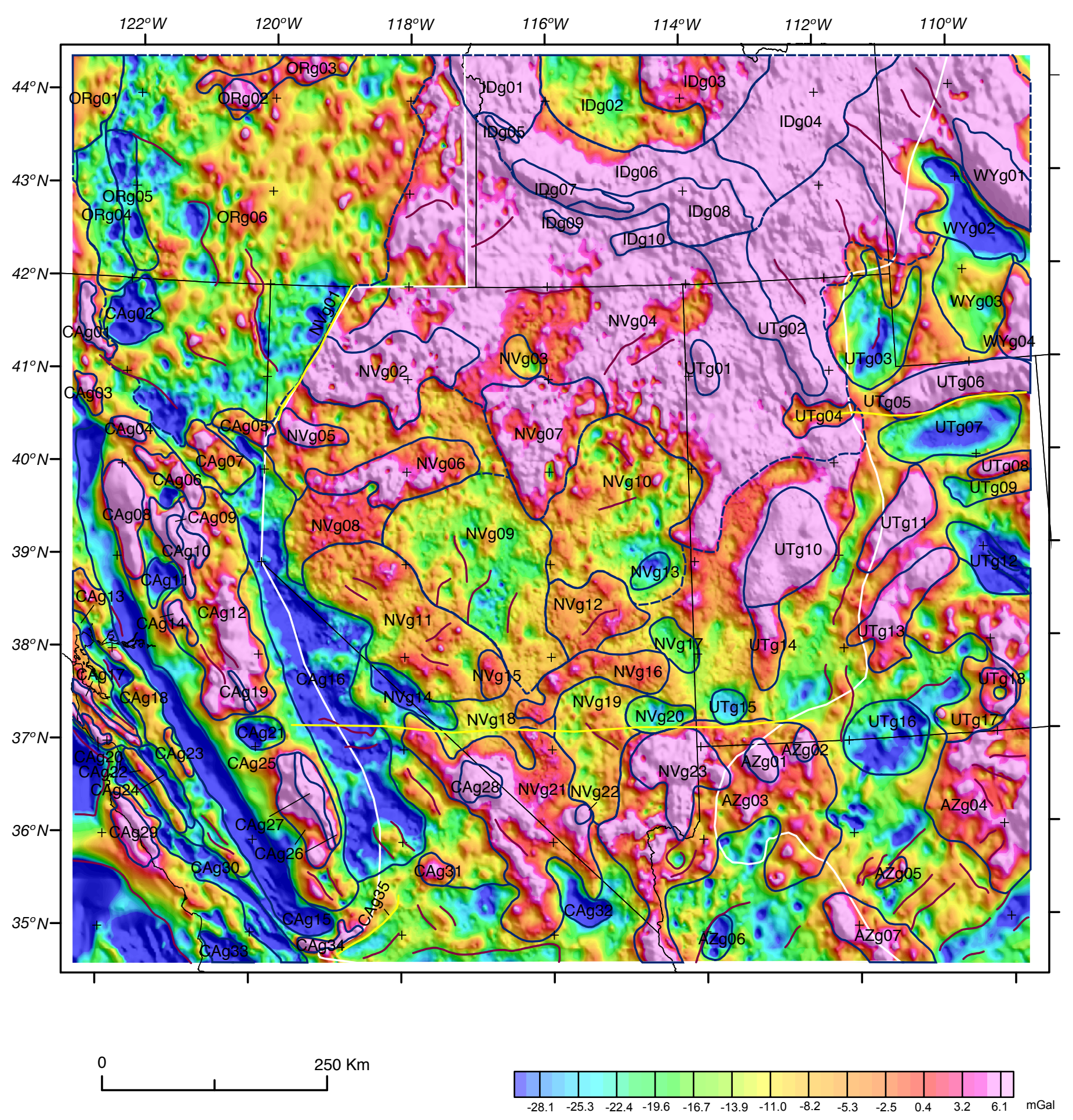

Figure 2. Basement gravity terrane map of the Great Basin. Blue lines circumscribe labeled polygonal features described in Table 1; yellow lines show labeled linear features described in Table 1; red lines show undescribed linear trends. 

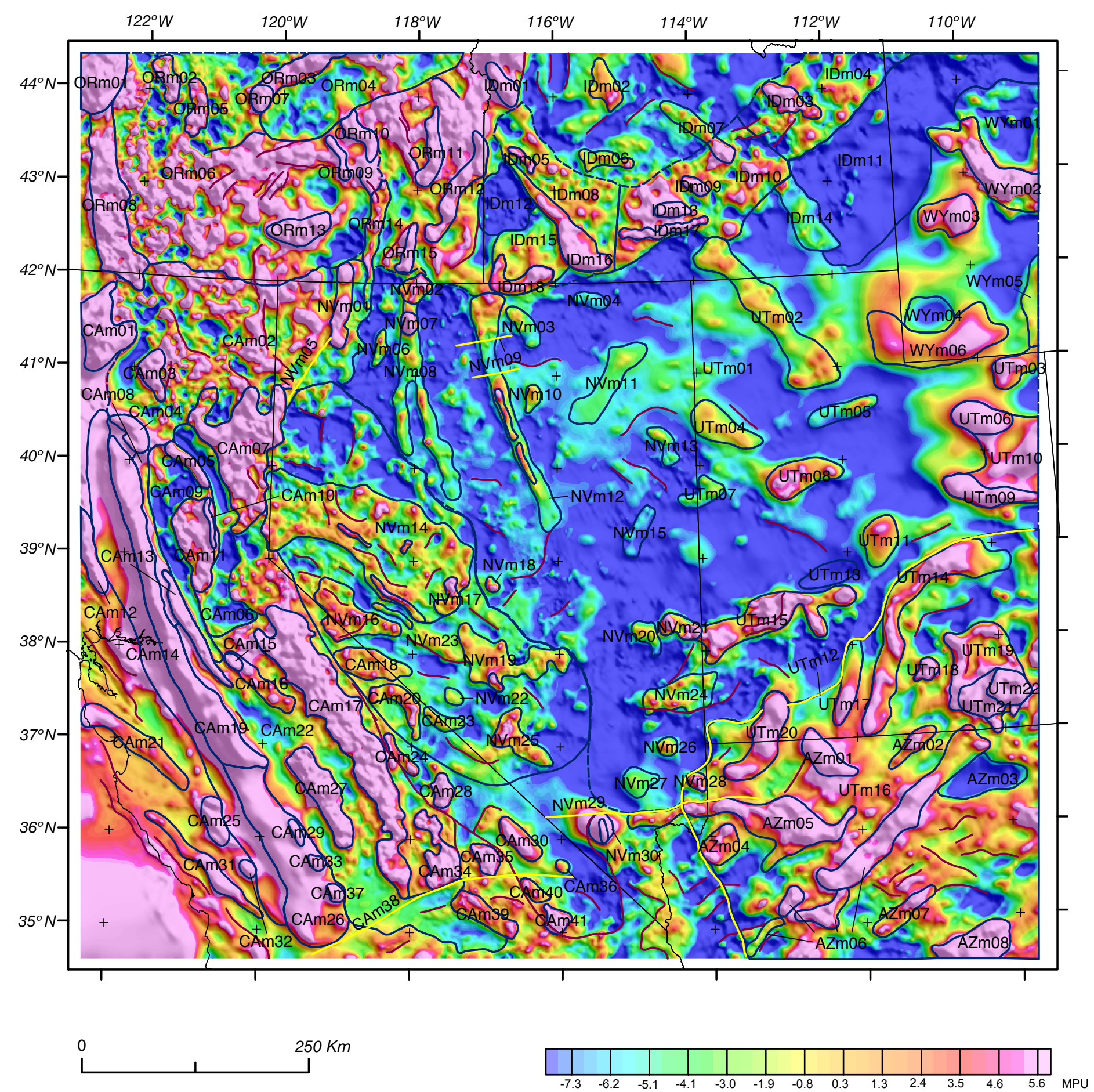

Figure 3. Magnetic potential (pseudogravity) terrane map of the Great Basin. Blue lines circumscribe labeled polygonal features described in Table 1; yellow lines show labeled linear features described in Table 1; red lines show undescribed linear trends. 

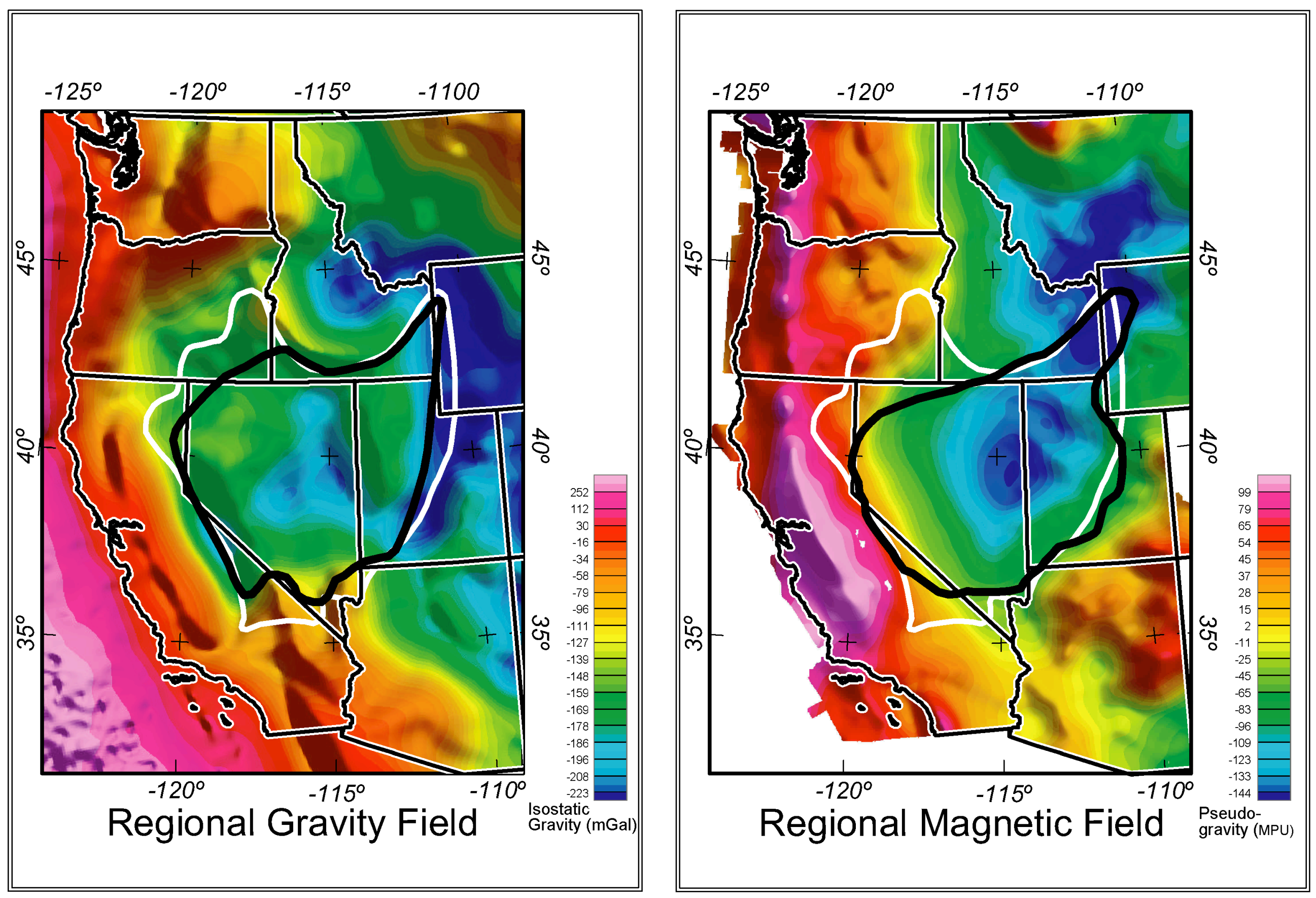

Figure 4. Regional isostatic gravity and pseudogravity field maps of the Great Basin. 


\begin{tabular}{|c|c|c|c|c|c|c|c|c|c|}
\hline Scale & ID & $\begin{array}{l}\mathrm{Hi} / \mathrm{lol} \\
\text { both }\end{array}$ & Terrane definition & Geologic province & Inferred source of anomaly & $\begin{array}{l}\text { Generalized } \\
\text { source rock }\end{array}$ & $\begin{array}{l}\text { Tectonic } \\
\text { setting }\end{array}$ & Province & References \\
\hline $\mathbf{R}$ & G1 & & $\begin{array}{l}\text { Regional long-wavelength gravity anomaly spans } \\
\text { entire GB and has bi-lateral symmetry } \\
\text { (resembling the form of a butterfly). Anomaly } \\
\text { appears best in isostatic gravity and topography, } \\
\text { but expressed also in pseudogravity (see regional } \\
\text { isostatic field map, figure 4). Corresponds with } \\
\text { regional magnetic low. }\end{array}$ & $\begin{array}{l}\text { Anomaly corresponds roughly to a } \\
\text { topographically high region of extended } \\
\text { crust associated with the GB Province. }\end{array}$ & $\begin{array}{l}\text { Anomaly reflects regionally extended, } \\
\text { hot and elevated crust. }\end{array}$ & varied & extension & Great Basin & $\begin{array}{l}\text { Fenneman, 1931; } \\
\text { Eaton and others, 1978; } \\
\text { Mabey and others, 1978; } \\
\text { Glen and others, 2000 }\end{array}$ \\
\hline $\mathbf{R}$ & M2 & & $\begin{array}{l}\text { Broadest regional pseudogravity feature } \\
\text { corresponding roughly with the extent of the GB } \\
\text { defined by topography (see regional } \\
\text { pseudogravity map, figure 4). Corresponds with } \\
\text { regional isostatic gravity low. }\end{array}$ & $\begin{array}{l}\text { Anomaly corresponds roughly to a } \\
\text { topographically high region of extended } \\
\text { crust associated with the GB Province. }\end{array}$ & $\begin{array}{l}\text { Anomaly reflects regionally extended, } \\
\text { hot and elevated crust. }\end{array}$ & varied & extension & Great Basin & $\begin{array}{l}\text { Fenneman, 1931; } \\
\text { Eaton and others, 1978; } \\
\text { Mabey and others, 1978; } \\
\text { Glen and others, 2000 }\end{array}$ \\
\hline 1 & AZgo1 & $\mathrm{H}$ & $\begin{array}{l}\text { Oval gravity high ( } 35 \mathrm{~km}) \text { that correlates with } \\
\text { magnetic anomaly UTm } 20 .\end{array}$ & $\begin{array}{l}\text { Located on } \mathrm{CP} \text { close to Hurricane Fault } \\
\text { zone. Corresponds in part with Q and } \mathrm{T} \\
\text { volcanic centers. }\end{array}$ & $\begin{array}{l}\text { Source is buried by Pz and Mz strata. } \\
\text { May be dense and magnetic buried } \\
\text { pC basement or Q and T volcanic } \\
\text { rocks. }\end{array}$ & $\begin{array}{l}\text { basement, } \\
\text { volcanic mafic }\end{array}$ & $\begin{array}{l}\text { uplift, stable } \\
\text { crust, } \\
\text { extension }\end{array}$ & $\begin{array}{l}\text { Colorado } \\
\text { Plateaus }\end{array}$ & \\
\hline 1 & AZg02 & $\mathbf{H}$ & $\begin{array}{l}\text { Oval gravity high }(35 \mathrm{~km}) \text { over north end of } \\
\text { magnetic anomaly AZm01. }\end{array}$ & $\begin{array}{l}\text { Located over a broader high on Kanab } \\
\text { Plateau. }\end{array}$ & $\begin{array}{l}\text { Source is buried by Pz and Mz strata. } \\
\text { Source may be dense and magnetic } \\
\text { buried pC basement. }\end{array}$ & basement & $\begin{array}{l}\text { uplift, stable } \\
\text { crust }\end{array}$ & $\begin{array}{l}\text { Colorado } \\
\text { Plateaus }\end{array}$ & \\
\hline I & AZg03 & $\mathrm{H}$ & $\begin{array}{l}\text { EW-elongate regional gravity high }(250 \times 125 \mathrm{~km}) \\
\text { containing some prominent large }(\sim 100 \mathrm{~km} \\
\text { diameter) highs. }\end{array}$ & $\begin{array}{l}\text { Northern part of feature corresponds to } \\
\text { CP/GB transition. Western part is entirely } \\
\text { within the GB. Northern and western } \\
\text { portions correspond closely with outcrops of } \\
\text { Pz strata. }\end{array}$ & Shallow pC-basement rocks. & basement & uplift & $\begin{array}{l}\text { Colorado } \\
\text { Plateaus }\end{array}$ & \\
\hline 1 & AZg04 & $\mathrm{H}$ & Broad gravity high (350x150km). & $\begin{array}{l}\text { Located over Pz, and thickest Mz strata in } \\
\text { AZ. coincides in part with the Black Mesa } \\
\text { Basin. }\end{array}$ & $\begin{array}{l}\text { Regionally elevated dense pC } \\
\text { basement rocks beneath CP. }\end{array}$ & basement & $\begin{array}{l}\text { uplift, stable } \\
\text { crust }\end{array}$ & $\begin{array}{l}\text { Colorado } \\
\text { Plateaus }\end{array}$ & Sumner, 1985 \\
\hline & AZg05 & $\mathrm{H}$ & $\begin{array}{l}\text { NE-trending gravity high }(50 \times 25 \mathrm{~km}) \text {. Edges } \\
\text { correspond to steep magnetic gradients. }\end{array}$ & $\begin{array}{l}\text { Located over T and Q volcanic rocks of the } \\
\text { San Francisco Peaks volcanic field. Trend is } \\
\text { similar to axes of monoclines in Mz strata } \\
\text { that may indicate step faults in basement. }\end{array}$ & $\begin{array}{l}\text { Uplifted dense pC basement or dense } \\
\text { intrusive rocks associated with } \mathrm{Cz} \\
\text { volcanic field. }\end{array}$ & $\begin{array}{l}\text { basement, } \\
\text { intrusive mafic }\end{array}$ & $\begin{array}{l}\text { uplift, stable } \\
\text { crust }\end{array}$ & $\begin{array}{l}\text { Colorado } \\
\text { Plateaus }\end{array}$ & \\
\hline I & AZg06 & L & $\begin{array}{l}\text { NE-trending gravity low }(100 \times 25 \mathrm{~km}) \text { within a } \\
\text { much broader gravity low. Corresponds with } \\
\text { magnetic low. }\end{array}$ & $\begin{array}{l}\text { Lies within the transition zone between GB } \\
\text { CP within a broader gravity low, over pC } \\
\text { basement and Mz granitic rocks. }\end{array}$ & $\begin{array}{l}\text { Low density, non-magnetic } \mathrm{pC} \\
\text { basement (silicic gneisses) and / or Mz } \\
\text { granitic rocks. }\end{array}$ & $\begin{array}{l}\text { basement, } \\
\text { intrusive silicic }\end{array}$ & uplift & $\begin{array}{l}\text { Colorado } \\
\text { Plateaus }\end{array}$ & Sumner, 1985 \\
\hline I & AZg07 & $\mathrm{H}$ & $\begin{array}{l}\text { Prominant NW-trending gravity high } \\
\text { (175x50km). Corresponds closely with a } \\
\text { magnetic low (not numbered). }\end{array}$ & $\begin{array}{l}\text { Located near the south edge of CP } \\
\text { (Mogollon Rim). }\end{array}$ & $\begin{array}{l}\text { Dense, nonmagnetic crystalline } \mathrm{pC}- \\
\text { basement. }\end{array}$ & basement & uplift & $\begin{array}{l}\text { Colorado } \\
\text { Plateaus }\end{array}$ & \\
\hline 1 & AZm01 & $\mathrm{H}$ & $\begin{array}{l}\text { NW-trending magnetic high }(75 \times 40 \mathrm{~km}) \text { within a } \\
\text { broader high of CP. North end corresponds to } \\
\text { gravity high AZg02. }\end{array}$ & Kaibab (Unikaret) Plateau (CP). & $\begin{array}{l}\text { Buried by Pz and Mz strata. Uplifted } \\
\text { magnetic pC basement. }\end{array}$ & basement & $\begin{array}{l}\text { uplift, stable } \\
\text { crust }\end{array}$ & $\begin{array}{l}\text { Colorado } \\
\text { Plateaus }\end{array}$ & \\
\hline 1 & AZm02 & $\mathrm{H}$ & $\begin{array}{l}\text { NE-trending elongate magnetic high }(75 \times 25 \mathrm{~km}) \\
\text { located within a much larger gravity low. }\end{array}$ & $\begin{array}{l}\text { Over Pz and Mz strata over the Paria } \\
\text { Plateau (CP). Parallels plunging anticline, } \\
\text { and is in line with the northeastern } \\
\text { extension of the Bright Angle Fault. }\end{array}$ & $\begin{array}{l}\text { Magnetic intrusive or faulted and } \\
\text { uplifted basement rocks. }\end{array}$ & $\begin{array}{l}\text { intrusive mafic, } \\
\text { basement }\end{array}$ & $\begin{array}{l}\text { stable crust, } \\
\text { uplift }\end{array}$ & $\begin{array}{l}\text { Colorado } \\
\text { Plateaus }\end{array}$ & \\
\hline 1 & AZm03 & L & $\begin{array}{l}\text { Prominent east-trending magnetic low }(75 \times 40 \mathrm{~km}) \\
\text { that is within a much larger region of gravity } \\
\text { high. }\end{array}$ & $\begin{array}{l}\text { Over flat-lying Pz and Mz strata of CP. } \\
\text { Corresponds to the NW part of the Black } \\
\text { Mesa Basin. }\end{array}$ & $\begin{array}{l}\text { Source is covered by Pz and Mz } \\
\text { strata. Possible uplift of non- } \\
\text { magnetic pC basement. }\end{array}$ & basement & $\begin{array}{l}\text { uplift, stable } \\
\text { crust }\end{array}$ & $\begin{array}{l}\text { Colorado } \\
\text { Plateaus }\end{array}$ & Sumner, 1985 \\
\hline 1 & AZm04 & H & $\begin{array}{l}\text { Equidimensional }(40 \mathrm{~km}) \text { magnetic high. Over a } \\
\text { complex gravity anomaly. Mostly within but } \\
\text { near the edge of a generally high magnetic } \\
\text { region. }\end{array}$ & $\begin{array}{l}\text { At west edge of CP. Includes Iron Mtn } \\
\text { volcanic rocks. }\end{array}$ & $\begin{array}{l}\text { Probable uplifted magnetic pC } \\
\text { basement or } \mathrm{Cz} \text { volcanic and } \\
\text { associated intrusive rocks. }\end{array}$ & $\begin{array}{l}\text { basement, } \\
\text { volcanic mafic, } \\
\text { intrusive mafic }\end{array}$ & $\begin{array}{l}\text { uplift, stable } \\
\text { crust }\end{array}$ & $\begin{array}{l}\text { Colorado } \\
\text { Plateaus }\end{array}$ & \\
\hline
\end{tabular}




\begin{tabular}{|c|c|c|c|c|c|c|c|c|c|}
\hline Scale & ID & $\begin{array}{l}\mathrm{Hi} / \mathrm{lo} / \\
\text { both }\end{array}$ & Terrane definition & Geologic province & Inferred source of anomaly & $\begin{array}{l}\text { Generalized } \\
\text { source rock }\end{array}$ & $\begin{array}{l}\text { Tectonic } \\
\text { setting }\end{array}$ & Province & References \\
\hline I & AZm05 & $\mathrm{H}$ & $\begin{array}{l}\text { EW-trending elongate magnetic anomaly high } \\
\text { (250km) that narrows at its west end (10km wide } \\
\text { extending for } \sim 150 \mathrm{~km} \text { ). East end widens to } \sim 75 \\
\mathrm{~km} \text {. }\end{array}$ & $\begin{array}{l}\text { East end includes a large part of Coconino } \\
\text { plateau. West end roughly corresponds } \\
\text { with trace of the Colorado River and Lake } \\
\text { Mead. Includes Mt Floyd volcanic field. }\end{array}$ & $\begin{array}{l}\text { Probable uplifted magnetic } \mathrm{pC} \\
\text { basement uplifted on east-trending } \\
\text { fault, or } \mathrm{Cz} \text { volcanic or intrusive } \\
\text { rocks. }\end{array}$ & $\begin{array}{l}\text { basement, } \\
\text { intrusive silicic, } \\
\text { volcanic silicic }\end{array}$ & uplift & $\begin{array}{l}\text { Colorado } \\
\text { Plateaus }\end{array}$ & Sumner, 1985 \\
\hline 1 & AZm06 & $\mathrm{H}$ & $\begin{array}{l}\text { NE-trending elongate magnetic high } \\
\text { (250kmx25km). SE-edge corresponds with } \\
\text { gravity lineations. Includes three small } \\
\text { anomalies at SW end. }\end{array}$ & $\begin{array}{l}\text { Southern part correlates with San Francisco } \\
\text { Peaks volcanic field. Straddles edge of CP. } \\
\text { Anomaly resembles that associated with the } \\
\text { Springerville volcanic field to SE. Trend is } \\
\text { parallel to the Colorado Lineament which } \\
\text { may be related to the Colorado Mineral Belt } \\
\text { located to the NE. Aligned along Mesa } \\
\text { Butte Fault. }\end{array}$ & $\begin{array}{l}\text { Magnetic } \mathrm{pC} \text { basement or } \mathrm{Cz} \text { igneous } \\
\text { rocks that exploited a fracture system } \\
\text { in the basement. }\end{array}$ & $\begin{array}{l}\text { basement, } \\
\text { intrusive mafic, } \\
\text { volcanic mafic }\end{array}$ & stable crust & $\begin{array}{l}\text { Colorado } \\
\text { Plateaus }\end{array}$ & Stewart and Crowell, 1992 \\
\hline I & AZm07 & $\mathrm{H}$ & $\begin{array}{l}\text { NE-trending elongate magnetic high (100x25km). } \\
\text { Corresponds with NE-trending linear gravity } \\
\text { features. }\end{array}$ & $\begin{array}{l}\text { Located in transition zone of CP and BR, } \\
\text { over Hackberry Miocene lava flows. }\end{array}$ & $\begin{array}{l}\text { Magnetic volcanic rocks and feeder } \\
\text { dikes. }\end{array}$ & volcanic mafic & extension & $\begin{array}{l}\text { Colorado } \\
\text { Plateaus }\end{array}$ & \\
\hline I & AZm08 & $\mathrm{H}$ & $\begin{array}{l}\text { NE-trending elongate magnetic high }(100 \times 25 \mathrm{~km}) \\
\text { located on a gravity low. }\end{array}$ & $\begin{array}{l}\text { Located in transition zone of CP and BR, on } \\
\text { pC (Apache group) rocks. }\end{array}$ & Magnetic pC basement. & basement & uplift & $\begin{array}{l}\text { Colorado } \\
\text { Plateaus }\end{array}$ & \\
\hline I & CAg01 & $\mathrm{H}$ & $\begin{array}{l}\text { Narrow roughly NS-trending gravity high } \\
(100 x 10 \mathrm{~km}) \text {. Located entirely within a magnetic } \\
\text { high. }\end{array}$ & $\begin{array}{l}\text { Anomaly located over complex of Mz } \\
\text { intrusive rocks, Pz and Mz metasedimentary } \\
\text { rocks, and ultramafic rocks that are part of } \\
\text { the Klamath Mtns. Gravity anomaly is } \\
\text { centered over a magnetic high (CAm01). }\end{array}$ & $\begin{array}{l}\text { Gravity anomaly mimics crescent- } \\
\text { shaped structural slivers, and is } \\
\text { probably due to a dense portion of } \\
\text { the eastern Klamath Mtns, probably } \\
\text { mafic ultramafic rocks of the Trinity } \\
\text { Ophiolite. }\end{array}$ & $\begin{array}{l}\text { intrusive mafic, } \\
\text { volcanic mafic, } \\
\text { ultramafic }\end{array}$ & accretion & Klamath Mtns & $\begin{array}{l}\text { LaFehr, 1966; Griscom,1980b; } \\
\text { Jachens and Griscom, 1985; } \\
\text { Jachens and others, } 1989\end{array}$ \\
\hline 1 & CAg02 & $\mathrm{L}$ & $\begin{array}{l}\text { Oval gravity low }(75 \mathrm{~km}) \text { within regionally } \\
\text { complex magnetic region (CAm } 02) \text {. }\end{array}$ & $\begin{array}{l}\text { Over } \mathrm{Q} \text { and } \mathrm{T} \text { volcanic arc terrain. Includes } \\
\text { Mount Shasta at its SW end. It is ringed on } \\
\text { its southwest half by Klamath Mtns Mz } \\
\text { intrusive rocks. }\end{array}$ & $\begin{array}{l}\text { Probably thick sequence of low } \\
\text { density volcanic rocks. }\end{array}$ & volcanic silicic & accretion & Cascade Mtns & Griscom,1980a \\
\hline 1 & CAg03 & $\mathrm{H}$ & $\begin{array}{l}\text { NW-elongate gravity high (50x20km). } \\
\text { Corresponds to a magnetic high region. }\end{array}$ & $\begin{array}{l}\text { Primarily on Pz limestones of the Klamath } \\
\text { Mtns. }\end{array}$ & \begin{tabular}{|l|} 
Probably due to dense, magnetic \\
mafic and ultramafic rocks of the \\
Trinity Ophiolite, though some of \\
gravity anomaly may be due to dense \\
carbonate rocks.
\end{tabular} & $\begin{array}{l}\text { ultramafic, } \\
\text { sedimentary } \\
\text { carbonate }\end{array}$ & accretion & Klamath Mtns & \\
\hline 1 & CAg04 & $\mathbf{H}$ & NW-trending moderate gravity high $(30 \times 20 \mathrm{~km})$. & Over $\mathrm{Q}$ and $\mathrm{T}$ volcanic rocks. & \begin{tabular}{|l|}
$\begin{array}{l}\text { Probably dense, magnetic } \\
\text { metamorphic or intrusive rocks of the } \\
\text { Sierra Nevada foothills and Klamath } \\
\text { Mtns. }\end{array}$ \\
\end{tabular} & $\begin{array}{l}\text { intrusive mafic, } \\
\text { metamorphic } \\
\text { mafic }\end{array}$ & accretion & Sierra Nevada & \\
\hline & CAg05 & $\mathbf{H}$ & $\begin{array}{l}\text { NW-trending gravity high }(75 \times 50 \mathrm{~km}) \text {. Located } \\
\text { over a prominant magnetic high. Feature looks } \\
\text { similar (in gravity and magnetics) to feature } \\
\text { (CAg07). }\end{array}$ & Over T volcanic rocks. & $\begin{array}{l}\text { Due to relatively dense and magnetic } \\
\text { Mz granitic T volcanic rocks similar to } \\
\text { feature CAg07. }\end{array}$ & $\begin{array}{l}\text { volcanic mafic, } \\
\text { intrusive silicic }\end{array}$ & $\begin{array}{l}\text { batholith, } \\
\text { extension }\end{array}$ & Great Basin & \\
\hline I & CAg06 & $\mathrm{H}$ & $\begin{array}{l}\text { NW-trending moderate gravity high (100x } 20 \mathrm{~km}) \\
\text { Located mostly over a magnetic low (unlabeled). }\end{array}$ & $\begin{array}{l}\text { Over Mz and Pz rocks of the western } \\
\text { foothills of the Sierra Nevada. }\end{array}$ & \begin{tabular}{|l|} 
Sources are various NW-trending Mz \\
and Pz intrusive and metamorphic \\
rocks in western Sierra Nevada \\
foothills. Anomaly likely due to \\
intrusive and metamorphic rocks.
\end{tabular} & $\begin{array}{l}\text { intrusive mafic, } \\
\text { metamorphic }\end{array}$ & accretion & Sierra Nevada & Burnett and Jennings, 1982 \\
\hline I & CAg07 & $\mathrm{H}$ & $\begin{array}{l}\text { NW-trending }(100 \times 50 \mathrm{~km}) \text { variable gravity high. } \\
\text { Located over a magnetic high region. Gravity } \\
\text { anomaly is similar to the main Sierra Nevada } \\
\text { batholith CAg12, though more magnetic. }\end{array}$ & $\begin{array}{l}\text { Over T volcanic rocks, Mz granitic and } \\
\text { metavolcanic rocks, and some Mz and } \mathrm{Pz} \\
\text { strata in the NW part of anomaly. }\end{array}$ & $\begin{array}{l}\text { Due to relatively dense and magnetic } \\
\text { Mz granitic or metavolcanic rocks, or } \\
\text { T volcanic rocks. }\end{array}$ & $\begin{array}{l}\text { volcanic mafic, } \\
\text { intrusive silicic }\end{array}$ & $\begin{array}{l}\text { batholith, } \\
\text { extension }\end{array}$ & Great Basin & \\
\hline 1 & CAg08 & $\mathrm{H}$ & $\begin{array}{l}\text { NW-trending prominent gravity high } \\
(150 \times 50 \mathrm{~km}) \text {. Forms northern end of prominent } \\
\text { magnetic high (CAm19). }\end{array}$ & $\begin{array}{l}\text { Entirely over north end of Great Valley. } \\
\text { Sutter Buttes lies at its center. }\end{array}$ & $\begin{array}{l}\text { May be dense, magnetic mafic } \\
\text { volcanic roots, obducted magnetic } \\
\text { oceanic crust or ultramafic rocks } \\
\text { (ophiolite). }\end{array}$ & $\begin{array}{l}\text { intrusive mafic, } \\
\text { volcanic mafic, } \\
\text { ultramafic }\end{array}$ & accretion & Great Valley & $\begin{array}{l}\text { Griscom 1973; } \\
\text { Cady, 1975; } \\
\text { Jachens and others, 1989; } \\
\text { Jachens and others, 1995 }\end{array}$ \\
\hline
\end{tabular}


Table 1: Geophysical Features of the Great Basin and parts of the surrounding provinces

\begin{tabular}{|c|c|c|c|c|c|c|c|c|c|}
\hline Scale & ID & $\begin{array}{l}\mathrm{Hi} / / \mathrm{o} / \\
\text { both }\end{array}$ & Terrane definition & Geologic province & Inferred source of anomaly & $\begin{array}{l}\text { Generalized } \\
\text { source rock }\end{array}$ & $\begin{array}{c}\text { Tectonic } \\
\text { setting }\end{array}$ & Province & References \\
\hline & CAg09 & $\mathrm{H}$ & $\begin{array}{l}\text { NS-trending prominent gravity high (40x25km). } \\
\text { Located within gravity anomaly CAg10, and } \\
\text { magnetic anomaly high CAm11. }\end{array}$ & $\begin{array}{l}\text { Largely over Mz granitic pluton, and some } \\
\text { Pz carbonates. }\end{array}$ & $\begin{array}{l}\text { May be due to dense, magnetic } \\
\text { intrusive rocks or perhaps dense, } \\
\text { magnetic, mafic and ultramafic } \\
\text { oceanic crust (Smartville Ophiolite). }\end{array}$ & $\begin{array}{l}\text { intrusive mafic, } \\
\text { ultramafic }\end{array}$ & $\begin{array}{l}\text { accretion, } \\
\text { batholith }\end{array}$ & Sierra Nevada & Oliver, 1980 \\
\hline 1 & CAg10 & $\mathbf{H}$ & $\begin{array}{l}\text { Prominent NW-trending gravity high } \\
(100 \times 40 \mathrm{~km}) \text {. }\end{array}$ & $\begin{array}{l}\text { Over Mz granitic plutons, } \mathrm{Pz} \text { carbonate and } \\
\text { ultramafic rocks. }\end{array}$ & $\begin{array}{l}\text { May be due to dense, magnetic } \\
\text { intrusive rocks or dense, magnetic, } \\
\text { mafic and ultramafic thrust slices in } \\
\text { Klamath Mtns and Sierra Nevada. } \\
\end{array}$ & $\begin{array}{l}\text { intrusive mafic, } \\
\text { ultramafic }\end{array}$ & $\begin{array}{l}\text { accretion, } \\
\text { batholith }\end{array}$ & Sierra Nevada & $\begin{array}{l}\text { LaFehr, 1966; } \\
\text { Jachens and Griscom, 1985; } \\
\text { Jachens and others, } 1989\end{array}$ \\
\hline 1 & CAg11 & $\mathrm{L}$ & Oval gravity low $(50 \mathrm{~km})$ & Over a Mz pluton. & Low density intrusive rocks. & intrusive silicic & batholith & $\begin{array}{l}\text { Great Valley, } \\
\text { Sierra Nevada }\end{array}$ & Oliver, 1980 \\
\hline 1 & CAg12 & $\mathbf{H}$ & NNW-trending gravity high $(275 \times 75 \mathrm{~km})$. & $\begin{array}{l}\text { Occurs over Pz and Mz meta-sedimentary, } \\
\text { metavolcanic, oceanic crustal rocks } \\
\text { (ophiolites), and granitic rocks. }\end{array}$ & $\begin{array}{l}\text { Dense mafic and ultramafic ophiolite } \\
\text { rocks. }\end{array}$ & $\begin{array}{l}\text { intrusive mafic, } \\
\text { volcanic mafic, } \\
\text { ultramafic }\end{array}$ & $\begin{array}{l}\text { accretion, } \\
\text { batholith }\end{array}$ & $\begin{array}{l}\text { Sierra Nevada, } \\
\text { Great Valley }\end{array}$ & Oliver, 1980 \\
\hline I & CAg13 & $\mathrm{L}$ & $\begin{array}{l}\text { Narrow NW-trending gravity low }(150 x<10 \mathrm{~km}) \\
\text { in line with gravity low CAg24. }\end{array}$ & $\begin{array}{l}\text { Follows along trace of the Hayward and } \\
\text { Rodgers Creek Faults. }\end{array}$ & $\begin{array}{l}\text { Low density valley fill in East Bay } \\
\text { Trough, and eastern Santa Clara and } \\
\text { Livermore Valleys. }\end{array}$ & $\begin{array}{l}\text { sedimentary } \\
\text { siliceous }\end{array}$ & $\begin{array}{l}\text { transcurrent, } \\
\text { depression }\end{array}$ & Coast Ranges & Roberts and Jachens, 1993 \\
\hline I & CAg14 & $\mathrm{H}$ & NW-trending gravity high $(50 \times 20 \mathrm{~km})$. & $\begin{array}{l}\text { Mostly over alluvium and Tertiary } \\
\text { sediments. }\end{array}$ & $\begin{array}{l}\text { May be due to dense mafic to } \\
\text { ultramafic intrusive bodies or to } \\
\text { dense metasedimentary rocks. }\end{array}$ & $\begin{array}{l}\text { intrusive mafic, } \\
\text { ultramafic, } \\
\text { metamorphic } \\
\text { mafic }\end{array}$ & accretion & $\begin{array}{l}\text { Great Valley, } \\
\text { Sierra Nevada }\end{array}$ & \\
\hline I & CAg15 & $\mathrm{L}$ & $\begin{array}{l}\text { Prominent, elongate NW-trending gravity low } \\
(700 \times 50 \mathrm{~km}) \text {. Strong gradients on eastern edge } \\
\text { and in parts on its western edge. Located over } \\
\text { magnetic low anomaly CAm14. }\end{array}$ & $\begin{array}{l}\text { Located at westernmost edge of Great } \\
\text { Valley. }\end{array}$ & $\begin{array}{l}\text { Likely source is low density valley } \\
\text { fill. }\end{array}$ & $\begin{array}{l}\text { sedimentary } \\
\text { siliceous }\end{array}$ & depression & Great Valley & $\begin{array}{l}\text { Byerly, 1966; } \\
\text { Suppe, 1979; } \\
\text { Oliver and Griscom, 1980; } \\
\text { Jachens and others, 1989 }\end{array}$ \\
\hline $\mathbf{R}$ & CAg16 & L & $\begin{array}{l}\text { Prominent NW-trending gravity low }(550 \times 75 \mathrm{~km}) . \\
\text { Corresponds with magnetic high anomaly } \\
\text { CAm17. }\end{array}$ & $\begin{array}{l}\text { Gravity anomaly is broader than the } \\
\text { corresponding magnetic anomaly (CAm17), } \\
\text { but also located mostly over eastern Sierra } \\
\text { Nevada batholith. }\end{array}$ & Magnetic, low density granitic rocks. & intrusive silicic & batholith & Sierra Nevada & $\begin{array}{l}\text { Oliver and Mabey, 1963; } \\
\text { Griscom and Oliver, 1980; } \\
\text { Jachens and others, 1989 }\end{array}$ \\
\hline $\mathbf{I}$ & CAg17 & $\mathrm{H}$ & $\begin{array}{l}\text { Prominent narrow NW-trending gravity high } \\
(>100 \times 5 \mathrm{~km}) \text {. Southern half is located over } \\
\text { magnetic high CAm21. }\end{array}$ & Over Franciscan Complex rocks. & $\begin{array}{l}\text { May be due to mafic and ultramafic } \\
\text { rocks of the Franciscan Complex, such } \\
\text { as greenstones of Permanente and } \\
\text { Headlands terranes. }\end{array}$ & $\begin{array}{l}\text { extrusive mafic, } \\
\text { intrusive mafic, } \\
\text { ultramafic }\end{array}$ & $\begin{array}{l}\text { transcurrent, } \\
\text { accretion }\end{array}$ & Coast Ranges & Jachens and Griscom, in press \\
\hline $\mathbf{R}$ & CAg18 & B & $\begin{array}{l}\text { Irregular, NW-trending, long, linear, moderate } \\
\text { gravity high (400x25km) defined by gravity lows } \\
\text { to the east (CAg15) and to the west } \\
(\mathrm{CAm} 23,24,30)\end{array}$ & $\begin{array}{l}\text { Eastern Coast Ranges. Varied moderately } \\
\text { high-density rocks within the San Andreas } \\
\text { Fault zone. }\end{array}$ & $\begin{array}{l}\text { Various sources whose alignment is } \\
\text { associated with accretion along } \\
\text { continental margin and with } \\
\text { subsequent strike-slip faulting within } \\
\text { the San Andreas Fault zone. } \\
\text { Anomaly is due to exposed Mz } \\
\text { bedrock surounded by low-density } \\
\text { Cz rocks and basin fill. }\end{array}$ & $\begin{array}{l}\text { sedimentary } \\
\text { siliceous, } \\
\text { ultramafic, } \\
\text { volcanic mafic }\end{array}$ & $\begin{array}{l}\text { transcurrent, } \\
\text { accretion, } \\
\text { depression, } \\
\text { uplift }\end{array}$ & Coast Ranges & \\
\hline L & CAg19 & $\mathbf{H}$ & $\begin{array}{l}\text { Narrow linear NNW-trending gravity high } \\
(50 \times 2 \mathrm{~km}) \text {. }\end{array}$ & $\begin{array}{l}\text { Occurs over Pz and Mz metasedimentary, } \\
\text { metavolcanic, ophiolite, and granitic rocks. }\end{array}$ & $\begin{array}{l}\text { Dense mafic and ultramafic ophiolite } \\
\text { rocks }\end{array}$ & $\begin{array}{l}\text { intrusive mafic, } \\
\text { volcanic mafic, } \\
\text { ultramafic }\end{array}$ & accretion & Sierra Nevada & Oliver, 1980 \\
\hline & CAg20 & $\mathrm{H}$ & NW-trending gravity high $(50 \times 10 \mathrm{~km})$ & $\begin{array}{l}\text { Located over Mz Ben Lomond pluton in the } \\
\text { Santa Cruz Mtns. }\end{array}$ & $\begin{array}{l}\text { Magnetic Logan Gabbro beneath Ben } \\
\text { Lomond pluton. }\end{array}$ & intrusive mafic & $\begin{array}{l}\begin{array}{l}\text { accretion, } \\
\text { transcurrent }\end{array} \\
\end{array}$ & Coast Ranges & Jachens and others, 1998 \\
\hline $\mathbf{L}$ & CAg21 & $\mathbf{L}$ & $\begin{array}{l}\text { Oval gravity low }(50 \times 40 \mathrm{~km}) \text { associated with a } \\
\text { magnetic low. Similar to other western Sierra } \\
\text { gravity belt lows (e.g. CAg11, and unamed low } \\
\text { south of anomaly CAg06). }\end{array}$ & $\begin{array}{l}\text { Occurs mainly over Mz granitic rocks and } \\
\text { some Mz metasedimentary, and T,Q } \\
\text { sedimentary rocks. }\end{array}$ & Low density silicic plutons & intrusive felsic & batholith & $\begin{array}{l}\text { Sierra Nevada, } \\
\text { Great Valley }\end{array}$ & Oliver and others, 1992 \\
\hline \multirow[t]{2}{*}{ I } & CAg22 & $\mathbf{H}$ & Narrow NW-trending gravity high $(125 \mathrm{x}<10 \mathrm{~km})$ & $\begin{array}{l}\text { Over Salinian Mz intrusive body. Eastern } \\
\text { edge is along San Andreas Fault. }\end{array}$ & \begin{tabular}{|l|} 
Relatively dense intrusive body \\
surrounded by low-density basin fill.
\end{tabular} & intrusive silicic & $\begin{array}{l}\text { transcurrent, } \\
\text { accretion }\end{array}$ & Coast Ranges & \\
\hline & CAg23 & $\mathrm{H}$ & $\begin{array}{l}\text { NW-trending gravity high }(60 \times 10 \mathrm{~km}) \text {. } \\
\text { Corresponds with a NW-trending elongate } \\
\text { magnetic high. }\end{array}$ & $\begin{array}{l}\text { Located over eastern edge of Coast Range } \\
\text { Franciscan complex - includes ultramafic } \\
\text { rocks (serpentines and eclogites). }\end{array}$ & $\begin{array}{l}\text { Due to Franciscan metasedimentary } \\
\text { rocks. }\end{array}$ & ultramafic & $\begin{array}{l}\text { accretion, } \\
\text { transcurrent }\end{array}$ & Coast Ranges & \\
\hline
\end{tabular}


Table 1: Geophysical Features of the Great Basin and parts of the surrounding provinces

\begin{tabular}{|c|c|c|c|c|c|c|c|c|c|}
\hline Scale & ID & $\begin{array}{l}\mathrm{Hi} / \mathrm{lol} \\
\text { both }\end{array}$ & Terrane definition & Geologic province & Inferred source of anomaly & $\begin{array}{l}\text { Generalized } \\
\text { source rock }\end{array}$ & $\begin{array}{l}\text { Tectonic } \\
\text { setting }\end{array}$ & Province & References \\
\hline 1 & CAg24 & $\mathrm{L}$ & Narrow NW-trending gravity low $(125 \mathrm{x}<10 \mathrm{~km})$ & $\begin{array}{l}\text { Located over Coyote valley Q and T valley } \\
\text { fill, along eastern side of San Andreas Fault. }\end{array}$ & Low density valley fill. & $\begin{array}{l}\begin{array}{l}\text { sedimentary } \\
\text { siliceous }\end{array} \\
\end{array}$ & $\begin{array}{l}\text { transcurrent, } \\
\text { depression }\end{array}$ & Coast Ranges & \\
\hline 1 & CAg25 & $\mathrm{H}$ & \begin{tabular}{|l|} 
Large, NNW-trending moderate gravity high \\
(600x150km) containing several prominant highs \\
(e.g. CAg12, CAg26) and lows (e.g. CAg21)
\end{tabular} & $\begin{array}{l}\text { Western part occurs over Great Valley } \\
\text { sediments. Eastern part includes granitic } \\
\text { rocks of the Sierra Nevada Batholith and Mz } \\
\text { and Pz metasedimentary rocks of the } \\
\text { western Sierra Nevada. }\end{array}$ & \begin{tabular}{|l|}
$\begin{array}{l}\text { Dense mafic and ultramafic ophiolite } \\
\text { rocks }\end{array}$ \\
\end{tabular} & $\begin{array}{l}\text { intrusive mafic, } \\
\text { volcanic mafic, } \\
\text { ultramafic }\end{array}$ & $\begin{array}{l}\text { accretion, } \\
\text { batholith }\end{array}$ & $\begin{array}{l}\text { Sierra Nevada, } \\
\text { Great Valley }\end{array}$ & Oliver and Griscom, 1980 \\
\hline L & CAg26 & $\mathbf{H}$ & $\begin{array}{l}\text { NNW-trending gravity high }(150 x 70 \mathrm{~km}) \text {. } \\
\text { Anomaly occurs largely over a magnetic low. }\end{array}$ & $\begin{array}{l}\text { Western part of anomaly occurs over } \\
\text { sediments of the Great Valley. Eastern edge } \\
\text { occurs over Sierra Nevada Batholith and Mz } \\
\text { and Pz metasedimentary rocks. }\end{array}$ & $\begin{array}{l}\text { Dense mafic and ultramafic ophiolite } \\
\text { rocks. }\end{array}$ & $\begin{array}{l}\text { intrusive mafic, } \\
\text { volcanic mafic, } \\
\text { ultramafic }\end{array}$ & accretion & $\begin{array}{l}\text { Sierra Nevada, } \\
\text { Great Valley }\end{array}$ & Oliver and Griscom, 1980 \\
\hline $\mathbf{L}$ & CAg27 & $\mathrm{H}$ & $\begin{array}{l}\text { NNW-trending narrow gravity high }(125 \times 20 \mathrm{~km}) \\
\text { refered to as the Dinuba gravity lineament. } \\
\text { Anomally occurs over a magnetic high. }\end{array}$ & $\begin{array}{l}\text { Occurs at contact between Great Valley and } \\
\text { Sierra Nevada, mostly over mafic and } \\
\text { ultramafic Pz and Mz oceanic crustal rocks. }\end{array}$ & $\begin{array}{l}\text { Dense mafic and ultramafic ophiolite } \\
\text { rocks. }\end{array}$ & $\begin{array}{l}\text { intrusive mafic, } \\
\text { volcanic mafic, } \\
\text { ultramafic }\end{array}$ & accretion & $\begin{array}{l}\text { Sierra Nevada, } \\
\text { Great Valley }\end{array}$ & Oliver, 1980 \\
\hline I & CAg28 & $\mathbf{H}$ & $\begin{array}{l}\text { Prominent oval gravity high }(50 \mathrm{~km}) \text { located over } \\
\text { a magnetic low region. }\end{array}$ & $\begin{array}{l}\text { Located over the north end of Death Valley } \\
\text { over pC crystalline basement. }\end{array}$ & Dense crystalline basement. & basement mafic & uplift & Great Basin & Chapman and others, 1973 \\
\hline I & CAg29 & $\mathbf{H}$ & NW-trending gravity high $(100 \times 50 \mathrm{~km})$. & $\begin{array}{l}\text { Located over Franciscan assemblage } \\
\text { metamorphic (Sur Series) rocks to the west } \\
\text { of the Hosgri Fault and K Salinian granites } \\
\text { to the east. }\end{array}$ & $\begin{array}{l}\text { Salinian K granites, Franciscan (Sur } \\
\text { series) metasedimentary and } \\
\text { metavolcanic rocks. }\end{array}$ & \begin{tabular}{|l|} 
sedimentary \\
siliceous, \\
extrusive mafic, \\
metamorphic \\
mafic, intrusive \\
silicic
\end{tabular} & $\begin{array}{l}\text { transcurrent, } \\
\text { accretion }\end{array}$ & Coast Ranges & \\
\hline I & CAg30 & $\mathrm{L}$ & $\begin{array}{l}\text { Prominent NW-trending gravity low } \\
(>300 \times 25 \mathrm{~km}) \text {. Along it western edge is a magnetic } \\
\text { high }(\mathrm{CAm} 31) \text {. }\end{array}$ & $\begin{array}{l}\text { Follows the Salinas Valley Q fill and some } \\
\text { Micene sedimentary rocks. Anomaly is } \\
\text { bound on its western edge by Salinian } \\
\text { granitic basement. }\end{array}$ & Low density basin fill. & $\begin{array}{l}\text { sedimentary } \\
\text { siliceous }\end{array}$ & $\begin{array}{l}\text { transcurrent, } \\
\text { depression }\end{array}$ & Coast Ranges & $\begin{array}{l}\text { Chapman and Griscom, 1980; } \\
\text { Jachens and others, } 1989\end{array}$ \\
\hline 1 & CAg32 & $\mathrm{L}$ & $\begin{array}{l}\text { Prominent NW-trending gravity low }(100 \times 40 \mathrm{~km}) \\
\text { located over a magnetic low region. A small oval } \\
\text { magnetic high occurs at its north end. }\end{array}$ & $\begin{array}{l}\text { Located mostly over Mz Tectonia batholith, } \\
\text { Mz Kingston Peak intrusive rocks. }\end{array}$ & Low density intrusive body. & intrusive silicic & extension & $\begin{array}{l}\text { Great Basin, } \\
\text { Mojave }\end{array}$ & Healey, 1970 \\
\hline & CAg33 & L & $\begin{array}{l}\text { NW-trending gravity low }(>50 \times 50 \mathrm{~km}) \text {. Located } \\
\text { immediately west of a similarly shaped moderate } \\
\text { to low magnetic ridge. }\end{array}$ & $\begin{array}{l}\text { Located over } \mathrm{T} \text { and } \mathrm{K} \text { sediments of the Santa } \\
\text { Maria Basin. }\end{array}$ & Low density sedimentary rocks. & $\begin{array}{l}\text { sedimentary } \\
\text { siliceous }\end{array}$ & $\begin{array}{l}\text { depression, } \\
\text { transcurrent }\end{array}$ & Coast Ranges & \\
\hline & CAg34 & $\mathrm{H}$ & Narrow ENE-trending gravity high $(75 \times 20 \mathrm{~km})$. & $\begin{array}{l}\text { Located just north of the Garlock Fault over } \\
\text { Mz granitic rocks. }\end{array}$ & Dense granitic rocks. & intrusive silicic & batholith & $\begin{array}{l}\text { Coast Ranges, } \\
\text { Sierra Nevada, } \\
\text { Great Valley }\end{array}$ & \\
\hline I & CAg35 & B & $\begin{array}{l}\text { ENE-trending linear feature }(\sim 100 \mathrm{~km}) \text { dividing a } \\
\text { gravity high to the north and a low to the south. } \\
\text { Approximately corresponds with trace of the } \\
\text { Garlock Fault. Corresponds with magnetic } \\
\text { anomaly CAm38. }\end{array}$ & $\begin{array}{l}\text { Left-lateral fault bounding southern edges } \\
\text { of Coast Ranges, Great Valley, and Sierra } \\
\text { Nevada with the Mojave desert. }\end{array}$ & $\begin{array}{l}\text { Edges of anomalies associated with } \\
\text { Coast Ranges, Great Valley, Sierra } \\
\text { Nevada, and Mojave Desert. }\end{array}$ & & transcurrent & $\begin{array}{l}\text { Great Basin, } \\
\text { Mojave }\end{array}$ & Davis and Burchfiel, 1973 \\
\hline 1 & CAm01 & $\mathrm{H}$ & $\begin{array}{l}\text { Irregular shaped prominent magnetic high (75 } \\
\text { EW x 100km NS) that entirely encompasses the } \\
\text { associated gravity anomaly (CAg01). }\end{array}$ & \begin{tabular}{|l|} 
Eastern lobe of magnetic anomaly \\
corresponds with Mt Shasta. Bulk of \\
magnetic anomaly located over complex of \\
Mz intrusive rocks, Pz and Mz \\
metasedimentary rocks, and ultramafic \\
rocks that are part of the Klamath Mtns. \\
Anomaly corresponds mostly with a gravity \\
high (CAg01).
\end{tabular} & \begin{tabular}{|l|} 
Magnetic anomaly sources are varied. \\
Mostly due to magnetic intrusive \\
rocks of the Klamath Mtns. SE lobe of \\
magnetic anomaly may be due to \\
magnetic volcanic rocks of Mt Shasta.
\end{tabular} & $\begin{array}{l}\text { intrusive mafic, } \\
\text { volcanic mafic, } \\
\text { ultramafic }\end{array}$ & accretion & Klamath Mtns & $\begin{array}{l}\text { LaFehr, 1966; } \\
\text { Griscom, 1980b; } \\
\text { Blakely and others, 1985; } \\
\text { Jachens and others, 1989 }\end{array}$ \\
\hline
\end{tabular}


Table 1: Geophysical Features of the Great Basin and parts of the surrounding provinces

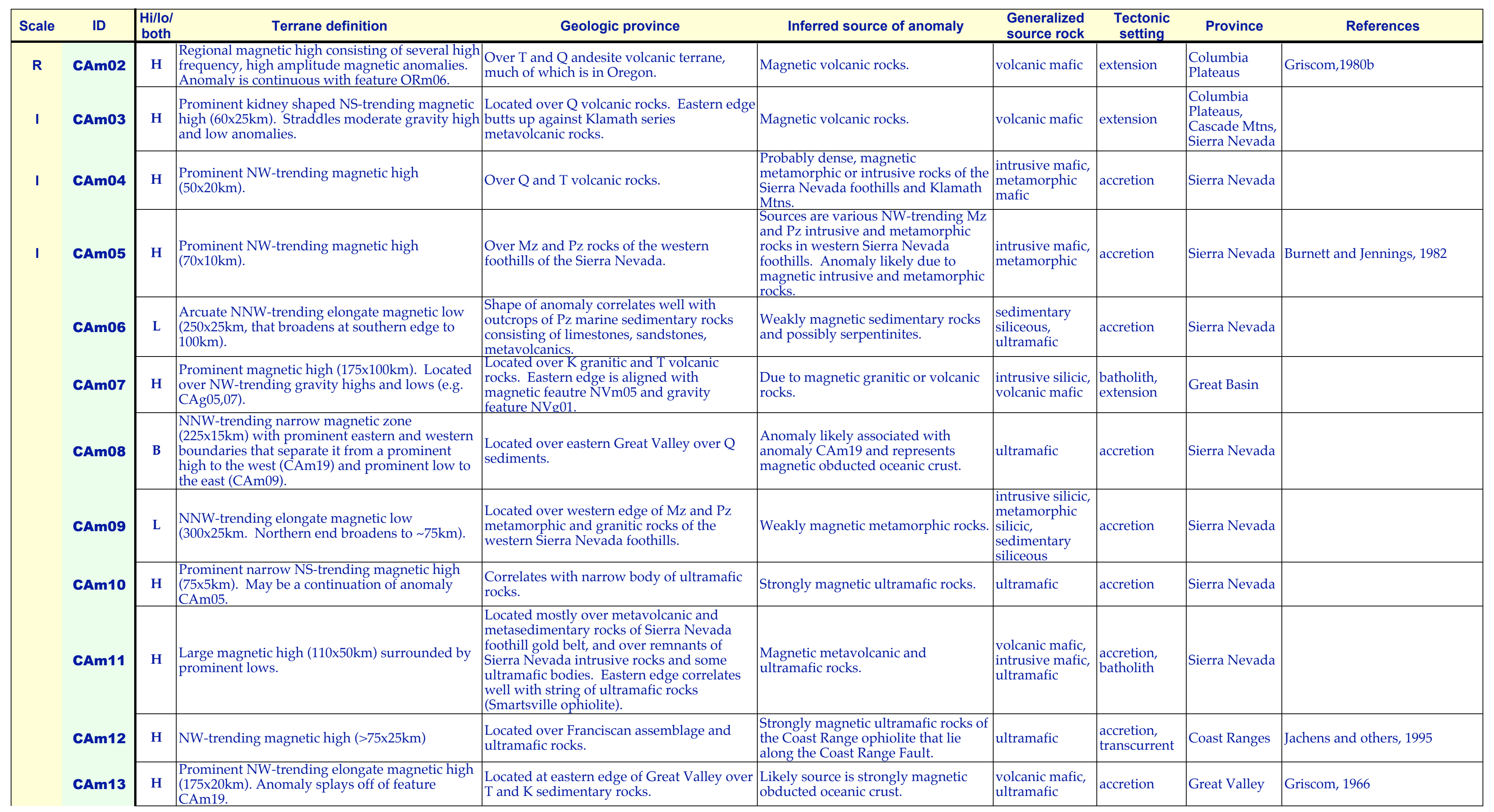




\begin{tabular}{|c|c|c|c|c|c|c|c|c|c|}
\hline Scale & ID & $\begin{array}{l}\mathrm{Hi} / \mathrm{lo} / \\
\text { both }\end{array}$ & Terrane definition & Geologic province & Inferred source of anomaly & $\begin{array}{l}\text { Generalized } \\
\text { source rock }\end{array}$ & $\begin{array}{l}\text { Tectonic } \\
\text { setting }\end{array}$ & Province & References \\
\hline \multirow[t]{3}{*}{1} & CAm14 & $\mathrm{L}$ & \begin{tabular}{|l|} 
Elongate NW-trending magnetic low region \\
directly west of prominent magnetic high \\
(CAm19). Its western edge (less well defined) \\
consists of a series of NW-trending discontinuous \\
gradients. Located over gravity low anomaly \\
CAg15.
\end{tabular} & $\begin{array}{l}\text { Located at westernmost edge of Great } \\
\text { Valley. }\end{array}$ & $\begin{array}{l}\text { Likely source is weakly magnetic } \\
\text { valley fill. }\end{array}$ & $\begin{array}{l}\text { sedimentary } \\
\text { siliceous }\end{array}$ & depression & Great Valley & $\begin{array}{l}\text { Byerly, 1966; } \\
\text { Suppe, 1979; } \\
\text { Oliver and Griscom, 1980; } \\
\text { Jachens and others, 1989 }\end{array}$ \\
\hline & CAm15 & $\mathrm{H}$ & Oval magnetic high $(25 \times 20 \mathrm{~km})$. & $\begin{array}{l}\text { Located over Pz metamorphic, } \mathrm{Q} \text { volcanic } \\
\text { and ultramafic rocks along the Bear Mt Fault } \\
\text { Zone. }\end{array}$ & $\begin{array}{l}\text { Likely due to strongly magnetic } \\
\text { ultramafic rocks. }\end{array}$ & ultramafic & accretion & Sierra Nevada & \\
\hline & CAm16 & $\mathrm{H}$ & $\begin{array}{l}\text { Long NW-trending magnetic high }(100 \times 20 \mathrm{~km}) \text {. } \\
\text { Anomally is mostly associated with a much } \\
\text { broader gravity high (CAg12). }\end{array}$ & $\begin{array}{l}\text { Occurs over Mz and Pz metasedimentary } \\
\text { rocks, granitic rocks, ophiolites and } \\
\text { serpentinites along the Bear Mt fault zone } \\
\text { and related faults. }\end{array}$ & $\begin{array}{l}\text { Dense mafic and ultramafic ophiolite } \\
\text { rocks. }\end{array}$ & $\begin{array}{l}\text { intrusive mafic, } \\
\text { ultramafic }\end{array}$ & accretion & Sierra Nevada & \\
\hline $\mathbf{R}$ & CAm17 & $\mathbf{H}$ & $\begin{array}{l}\text { Prominent NW-trending magnetic high } \\
\text { (50x400km).Corresponds with gravity low } \\
\text { anomaly CAg16. }\end{array}$ & $\begin{array}{l}\text { Magnetic anomaly located over eastern } \\
\text { Sierra Nevada batholith. Gravity anomaly } \\
\text { (CAg16) is broader, but also located mostly } \\
\text { over eastern Sierra Nevada batholith. }\end{array}$ & Magnetic, low density granitic rocks. & intrusive silicic & batholith & Sierra Nevada & $\begin{array}{l}\text { Oliver and Mabey, 1963; } \\
\text { Griscom and Oliver, 1980; } \\
\text { Jachens and others, } 1989\end{array}$ \\
\hline I & CAm18 & $\mathrm{H}$ & $\begin{array}{l}\text { Oblate EW trending moderate magnetic high } \\
(100 \times 40 \mathrm{~km}) .\end{array}$ & $\begin{array}{l}\text { Northeast of Long Valley Caldera, over } \\
\text { Benton Range and north end of White Mtns, } \\
\text { over Q volcanic and Mz intrusive rocks. }\end{array}$ & $\begin{array}{l}\text { Magnetic volcanic and intrusive } \\
\text { rocks. }\end{array}$ & $\begin{array}{l}\text { volcanic mafic, } \\
\text { intrusive mafic }\end{array}$ & $\begin{array}{l}\text { extension, } \\
\text { batholith }\end{array}$ & Great Basin & Stewart and Carlson, 1978 \\
\hline $\mathbf{R}$ & CAm19 & H & $\begin{array}{l}\text { Prominent NW-trending magnetic high } \\
\text { (450x40km). Strong gradients on its eastern edge } \\
\text { and in parts on its western edge. Corresponds } \\
\text { with subdued gravity high CAg25. Continuous } \\
\text { with magnetic anomaly CAm26. }\end{array}$ & $\begin{array}{l}\text { Located over basin fill of Great Valley. } \\
\text { Anomaly trends parallel to Great Valley. }\end{array}$ & $\begin{array}{l}\text { Slab of strongly magnetic obducted } \\
\text { oceanic crust and mantle. }\end{array}$ & $\begin{array}{l}\text { volcanic mafic, } \\
\text { ultramafic }\end{array}$ & accretion & Great Valley & $\begin{array}{l}\text { Griscom, 1966; } \\
\text { Cady, 1975; } \\
\text { Griscom and Jachens, 1990; } \\
\text { Jachens and others, } 1995\end{array}$ \\
\hline \multirow[t]{3}{*}{ I } & CAm20 & $\mathbf{H}$ & Arcuate shaped magnetic high $(75 \times 10 \mathrm{~km})$. & $\begin{array}{l}\text { Located over Jurassic Cottonwood pluton. } \\
\text { May be related to the Sylvania pluton } \\
\text { which has same age and composition. A } \\
\text { fault separates and may have offset these } \\
\text { plutons. }\end{array}$ & Magnetic intrusive rocks. & $\begin{array}{l}\text { intrusive mafic, } \\
\text { intrusive silicic }\end{array}$ & $\begin{array}{l}\text { extension, } \\
\text { batholith }\end{array}$ & Great Basin & McKee and Nelson, 1967 \\
\hline & CAm21 & $\mathrm{H}$ & $\begin{array}{l}\text { NW-trending moderate magnetic high } \\
\text { (150x25km). NW end corresponds with NW- } \\
\text { trending elongate gravity high (CAg17). SE end } \\
\text { corresponds with NW-trending gravity low } \\
\text { (CAg24). }\end{array}$ & $\begin{array}{l}\text { Southern end follows the Santa Clara Valley, } \\
\text { and is bound on the west by the San } \\
\text { Andreas Fault. In the north, the anomaly } \\
\text { crosses the San Andreas Fault and runs } \\
\text { offshore. }\end{array}$ & \begin{tabular}{|l|} 
Anomaly is caused by 3 different \\
sources in 3 different fault blocks. \\
Western end is offshore, west of the \\
San Gregorio Fault and is likely \\
caused by Coast Range ophiolite \\
rocks ocurring in Franciscan terrane. \\
Central part is caused by deeply \\
buried, concealed Logan gabbro of \\
likely ophiolite origin. Southeastern \\
part is due to slivers of Coast range \\
ophiolite rocks in Franciscan terrane.
\end{tabular} & $\begin{array}{l}\text { intrusive mafic, } \\
\text { ultramafic }\end{array}$ & $\begin{array}{l}\text { transcurrent, } \\
\text { accretion }\end{array}$ & Coast Ranges & Jachens and others, 1998 \\
\hline & CAm22 & $\mathbf{L}$ & $\begin{array}{l}\text { Long NNW-trending magnetic low }(500 \times 75 \mathrm{~km}) \\
\text { with internal magnetic highs (e.g. CAm27). } \\
\text { Southern portion of anomaly crosses the Garlock } \\
\text { Fault (CAm38) and is offset to the east by } \sim 75 \mathrm{~km} \text {. } \\
\end{array}$ & $\begin{array}{l}\text { Western part of anomaly occurs over Great } \\
\text { Valley sediments and Mz and Pz } \\
\text { metasedimentary rocks. Eastern, central and } \\
\text { southern parts occur over Sierra Nevada } \\
\text { batholith. Southern most part of anomaly, } \\
\text { south of the Garlock Fault, occurs over } \\
\text { granitic rocks and valley fill of the Mojave } \\
\text { Desert. }\end{array}$ & $\begin{array}{l}\text { Weakly magnetic sedimentary and } \\
\text { granitic rocks. }\end{array}$ & $\begin{array}{l}\text { intrusive silicic, } \\
\text { sedimentary } \\
\text { siliceous }\end{array}$ & $\begin{array}{l}\text { batholith, } \\
\text { depression }\end{array}$ & $\begin{array}{l}\text { Sierra Nevada, } \\
\text { Great Valley }\end{array}$ & \\
\hline \multirow[t]{2}{*}{1} & CAm23 & $\mathbf{H}$ & $\begin{array}{l}\text { Small WNW-trending moderate elongate } \\
\text { magnetic high }(40 \times 10 \mathrm{~km}) \text { on the CA-NV border. }\end{array}$ & Located over Jurassic Sylvania pluton. & Magnetic intrusive rocks. & $\begin{array}{l}\text { intrusive mafic, } \\
\text { intrusive silicic }\end{array}$ & $\begin{array}{l}\text { extension, } \\
\text { batholith }\end{array}$ & Great Basin & McKee, 1968 \\
\hline & CAm24 & H & Elongate NW-trending magnetic high $(60 \times 10 \mathrm{~km})$ & Located over Mz pluton in the Inyo Mtns. & Magnetic intrusive rocks. & $\begin{array}{l}\text { intrusive mafic, } \\
\text { intrusive silicic }\end{array}$ & $\begin{array}{l}\text { extension, } \\
\text { batholith }\end{array}$ & Great Basin & \\
\hline
\end{tabular}


Table 1: Geophysical Features of the Great Basin and parts of the surrounding provinces

\begin{tabular}{|c|c|c|c|c|c|c|c|c|c|}
\hline Scale & ID & $\begin{array}{l}\mathrm{H} i / \mathrm{lo} / \\
\text { both }\end{array}$ & Terrane definition & Geologic province & Inferred source of anomaly & $\begin{array}{l}\text { Generalized } \\
\text { source rock }\end{array}$ & $\begin{array}{c}\text { Tectonic } \\
\text { setting }\end{array}$ & Province & References \\
\hline \multirow{6}{*}{$\mathbf{R}$} & \multirow[b]{2}{*}{ CAm26 } & $\mathbf{H}$ & NNW-trending magnetic high $(60 \times 20 \mathrm{~km})$. & $\begin{array}{l}\text { Located near the San Andreas Fault zone } \\
\text { largely over T sedimentary and Franciscan } \\
\text { assemblage rocks. NE of the San Andreas } \\
\text { Fault, the main part of anomaly is over a } \\
\text { serpentinite diapir (New Idria Dome) and a } \\
\text { largely concealed serpentinite, flat-lying } \\
\text { body that extends SE to Table Mt. Southwest } \\
\text { of the San Andreas Fault, at the south end of } \\
\text { the anomaly, the anomaly is located over } \\
\text { Cretaceous intrusive rocks. }\end{array}$ & $\begin{array}{l}\text { Serpentinites and magnetic intrusive } \\
\text { rocks. }\end{array}$ & $\begin{array}{l}\text { ultramafic, } \\
\text { intrusive mafic, } \\
\text { intrusive silicic }\end{array}$ & $\begin{array}{l}\text { transcurrent, } \\
\text { accretion, } \\
\text { batholith }\end{array}$ & Coast Ranges & \\
\hline & & $\mathrm{H}$ & $\begin{array}{l}\text { Prominent NW-trending magnetic high } \\
(200 \times 50 \mathrm{~km}) \text {. Corresponds with subdued gravity } \\
\text { high CAg25. Continuous with magnetic anomaly } \\
\text { CAm19. }\end{array}$ & \begin{tabular}{|l|} 
Located over basin fill of Great Valley. \\
Anomaly trends parallel to Great Valley
\end{tabular} & $\begin{array}{l}\text { Slab of strongly magnetic obducted } \\
\text { oceanic crust and mantle. }\end{array}$ & $\begin{array}{l}\text { volcanic mafic, } \\
\text { ultramafic }\end{array}$ & accretion & Great Valley & $\begin{array}{l}\text { Cady, 1975; } \\
\text { Griscom and Jachens, 1990; } \\
\text { Jachens and others, } 1995\end{array}$ \\
\hline & CAm27 & $\mathbf{H}$ & $\begin{array}{l}\text { Long linear NW-trending magnetic high } \\
(125 \times 25 \mathrm{~km}) \text { corresponding to the Dinuba gravity } \\
\text { high }(\mathrm{CAg} 27) \text {. }\end{array}$ & \begin{tabular}{|l|} 
Occurs at contact between Great Valley and \\
Sierra Nevada, mostly over mafic and \\
ultramafic Pz and Mz oceanic crustal rocks.
\end{tabular} & $\begin{array}{l}\text { Magnetic mafic and ultramafic } \\
\text { ophiolite rocks. }\end{array}$ & $\begin{array}{l}\text { intrusive mafic, } \\
\text { volcanic mafic, } \\
\text { ultramafic }\end{array}$ & accretion & $\begin{array}{l}\text { Sierra Nevada, } \\
\text { Great Valley }\end{array}$ & \\
\hline & CAm28 & $\mathbf{H}$ & Prominent magnetic high (40x25km). & $\begin{array}{l}\text { Located over Mz pluton (Hunter Mt } \\
\text { batholith) in the central Panamint Range. }\end{array}$ & Magnetic intrusive rocks. & $\begin{array}{l}\text { intrusive mafic, } \\
\text { intrusive silicic }\end{array}$ & $\begin{array}{l}\text { extension, } \\
\text { batholith }\end{array}$ & Great Basin & $\begin{array}{l}\text { Mabey, 1961; } \\
\text { Chen and Moore, } 1982\end{array}$ \\
\hline & CAm29 & $\mathbf{H}$ & Small NW-trending magnetic high (10x20km). & $\begin{array}{l}\text { Located over eastern Great Valley and } \\
\text { within anomaly CAm } 26 .\end{array}$ & $\begin{array}{l}\text { May be a shallow sliver of obducted } \\
\text { magnetic oceanic crust. }\end{array}$ & $\begin{array}{l}\text { volcanic mafic, } \\
\text { intrusive mafic }\end{array}$ & accretion & Great Valley & \\
\hline & CAm30 & $\mathbf{H}$ & $\begin{array}{l}\text { Prominent elongate NW-trending magnetic high } \\
(70 \times 25 \mathrm{~km}) .\end{array}$ & $\begin{array}{l}\text { Located over T intrusive, volcanic, and } \\
\text { possibly pC crystalline basement rocks in } \\
\text { the Black Mtns. }\end{array}$ & $\begin{array}{l}\text { Likely due to magnetic intrusive, } \\
\text { volcanic and / or basement rocks. }\end{array}$ & $\begin{array}{l}\text { intrusive mafic, } \\
\text { volcanic mafic, } \\
\text { basement mafic }\end{array}$ & extension & Great Basin & Griscom, 1966 \\
\hline \multirow[t]{7}{*}{ I } & CAm31 & $\mathrm{H}$ & Narrow NW-trending magnetic high (100x10km). & $\begin{array}{l}\text { Located along fault traces of the San } \\
\text { Andreas Fault zone, over K granitic rocks of } \\
\text { the La Panza Range. }\end{array}$ & Magnetic intrusive rocks. & intrusive silicic & $\begin{array}{l}\text { transcurrent, } \\
\text { accretion }\end{array}$ & Coast Ranges & \\
\hline & CAm32 & $\mathrm{H}$ & Small oval magnetic high $(20 \mathrm{~km})$. & $\begin{array}{l}\text { Located over the eastern Coast Ranges on } \\
\text { the San Andreas Fault SE of Parkfield over T } \\
\text { sedimentary rocks, and near an outcrop of } \\
\text { Cretaceous granite. }\end{array}$ & $\begin{array}{l}\text { Inferred to be due to buried body of } \\
\text { magnetic serpentinite. }\end{array}$ & ultramafic & $\begin{array}{l}\text { transcurrent, } \\
\text { accretion }\end{array}$ & Coast Ranges & Hanna and others, 1972 \\
\hline & CAm33 & $\mathbf{H}$ & Small oval magnetic high (20km). & $\begin{array}{l}\text { Located over eastern part of Great Valley } \\
\text { and within anomaly CAm26. }\end{array}$ & $\begin{array}{l}\text { May be a shallow sliver of obducted } \\
\text { magnetic oceanic crust. }\end{array}$ & $\begin{array}{l}\text { ultramafic, } \\
\text { intrusive mafic }\end{array}$ & accretion & Great Valley & \\
\hline & CAm34 & $\mathrm{H}$ & NS-oriented magnetic high (100x50km). & $\begin{array}{l}\text { Located over Mz pluton associated with the } \\
\text { southern Sierra Nevada batholith over the } \\
\text { Argus Range. }\end{array}$ & Magnetic intrusive rocks. & intrusive mafic & $\begin{array}{l}\text { extension, } \\
\text { batholith }\end{array}$ & Great Basin & \\
\hline & CAm35 & $\mathrm{H}$ & Small magnetic high $(40 \times 40 \mathrm{~km})$. & $\begin{array}{l}\text { Located mostly over } \mathrm{T} \text { and } \mathrm{Q} \text { volcanic and } \\
\text { some Mz granitic rocks. Includes some } \\
\text { outcrop of pC rocks. }\end{array}$ & Magnetic volcanic or intrusive rocks & $\begin{array}{l}\text { volcanic mafic, } \\
\text { intrusive mafic }\end{array}$ & $\begin{array}{l}\text { extension, } \\
\text { batholith }\end{array}$ & Mojave Desert & \\
\hline & CAm36 & $\mathrm{H}$ & Small oval magnetic high $(10 \mathrm{~km})$. & Located over a T intrusive body. & Magnetic intrusive rocks. & intrusive mafic & extension & Great Basin & \\
\hline & CAm37 & $\mathrm{H}$ & Small oval magnetic high $(20 \mathrm{~km})$. & $\begin{array}{l}\text { Located over eastern part of Great Valley } \\
\text { and within anomaly CAm26. }\end{array}$ & $\begin{array}{l}\text { May be a shallow sliver of obducted } \\
\text { magnetic oceanic crust. }\end{array}$ & $\begin{array}{l}\text { ultramafic, } \\
\text { intrusive mafic }\end{array}$ & accretion & Great Valley & \\
\hline 1 & CAm38 & B & $\begin{array}{l}\text { ENE-trending linear feature }(\sim 300 \mathrm{~km}) \text { defined by } \\
\text { the alignment of truncated prominent magnetic } \\
\text { highs and lows of the Great Valley and Sierra } \\
\text { Nevada (e.g. CAm26, CAm22, CAm17, CAm34). } \\
\text { Also defined by left-lateral offset of prominant } \\
\text { magnetic low (CAm22) and magnetic highs } \\
\text { associated with Mz plutons (CAm17 and } \\
\text { CAm39). Left-lateral offset of anomalies appears } \\
\text { to be } \sim 75 \mathrm{~km} \text {. Approximately corresponds with } \\
\text { trace of the Garlock Fault. Corresponds with } \\
\text { gravity anomaly CAg35. }\end{array}$ & $\begin{array}{l}\text { Corresponds to left-lateral fault bounding } \\
\text { southern edges of Coast Ranges, Great } \\
\text { Valley, and Sierra Nevada with the Mojave } \\
\text { Desert. }\end{array}$ & $\begin{array}{l}\text { Edges of anomalies associated with } \\
\text { Coast Ranges, Great Valley, Sierra } \\
\text { Nevada, and Mojave Desert. }\end{array}$ & & transcurrent & $\begin{array}{l}\text { Great Basin, } \\
\text { Mojave }\end{array}$ & \\
\hline
\end{tabular}


Table 1: Geophysical Features of the Great Basin and parts of the surrounding provinces

\begin{tabular}{|c|c|c|c|c|c|c|c|c|c|}
\hline Scale & ID & $\begin{array}{l}\mathrm{Hi} / \mathrm{lol} \\
\text { both }\end{array}$ & Terrane definition & Geologic province & Inferred source of anomaly & $\begin{array}{l}\text { Generalized } \\
\text { source rock }\end{array}$ & $\begin{array}{c}\begin{array}{c}\text { Tectonic } \\
\text { setting }\end{array} \\
\end{array}$ & Province & References \\
\hline & CAm39 & $\mathbf{H}$ & $\begin{array}{l}\text { Elongate WNW-trending magnetic high } \\
(75 \times 25 \mathrm{~km}) \text {. }\end{array}$ & $\begin{array}{l}\text { Located mostly over Mz pluton in the } \\
\text { Mojave Desert. SE end sits over volcanic } \\
\text { rocks. }\end{array}$ & Magnetic intrusive rocks. & intrusive mafic & $\begin{array}{l}\text { extension, } \\
\text { batholith }\end{array}$ & Mojave Desert & \\
\hline & CAm40 & $\mathbf{H}$ & Small NW-trending magnetic high (30x10km). & Located over Mz pluton in Mojave Desert. & Magnetic intrusive rocks. & intrusive mafic & $\begin{array}{l}\text { extension, } \\
\text { batholith }\end{array}$ & Mojave Desert & \\
\hline & CAm41 & $\mathbf{H}$ & Small NW-trending magnetic high $(30 \times 20 \mathrm{~km})$. & $\begin{array}{l}\text { Located over Mz intrusive, Pz sedimentary } \\
\text { and pC basement rocks in the Mojave } \\
\text { Desert. }\end{array}$ & Magnetic intrusive or basement rocks. & $\begin{array}{l}\text { intrusive mafic, } \\
\text { basement mafic }\end{array}$ & batholith & Mojave Desert & \\
\hline I & IDg01 & $\mathbf{H}$ & $\begin{array}{l}\text { Broad NW-trending high containing several } \\
\text { smaller elongate anomalies (up to 200km). } \\
\text { Gravity terrane is north of, and broader than, the } \\
\text { magnetic terrane (IDm08). Contiguous with } \\
\text { IDg06 and IDg04. }\end{array}$ & $\begin{array}{l}\text { Veneer of alluvium overlying basaltic lava } \\
\text { flows. }\end{array}$ & Dense and magnetic basaltic rocks. & volcanic mafic & hotspot & $\begin{array}{l}\text { Snake River } \\
\text { Plain }\end{array}$ & $\begin{array}{l}\text { Mabey, 1976; } \\
\text { Mabey, 1982; } \\
\text { Sparlin and others, 1982; } \\
\text { Jachens and others, 1989 }\end{array}$ \\
\hline $\mathbf{R}$ & IDg02 & $\mathrm{L}$ & $\begin{array}{l}\text { Gravity low bounded on west and south by } \\
\text { western SRP high and on east by a gravity high } \\
\text { (IDg03). Located over a regional magnetic low } \\
\text { interspersed by isolated magnetic highs (e.g. } \\
\text { IDm02). }\end{array}$ & Granitic batholith. & $\begin{array}{l}\text { Low density, weakly magnetic } \\
\text { granitic rocks. }\end{array}$ & intrusive silicic & batholith & $\begin{array}{l}\text { Northern } \\
\text { Rocky Mtns }\end{array}$ & $\begin{array}{l}\text { Bott and Smithson, 1967; } \\
\text { Jachens and others, 1989; } \\
\text { Mabey, } 1986\end{array}$ \\
\hline I & IDg03 & $\mathbf{H}$ & $\begin{array}{l}\text { Uniform gravity high bounded on south and east } \\
\text { by intense gravity high of SRP and bounded on } \\
\text { west by ID batholith. Regional magnetic low } \\
\text { with local discrete highs. }\end{array}$ & $\begin{array}{l}\text { Northernmost extension of basin and range. } \\
\text { Mainly pC through upper Pz marine strata. } \\
\text { Thick cover of Eocene volcanic rocks. }\end{array}$ & $\begin{array}{l}\text { Dense sedimentary and basement } \\
\text { rocks. }\end{array}$ & $\begin{array}{l}\text { basement silicic, } \\
\text { basement } \\
\text { carbonate, } \\
\text { sedimentary } \\
\text { carbonate, } \\
\text { sedimentary } \\
\text { siliceous } \\
\end{array}$ & compression & $\begin{array}{l}\text { Northern } \\
\text { Rocky Mtns }\end{array}$ & \\
\hline \multirow[t]{2}{*}{ I } & IDg04 & $\mathbf{H}$ & $\begin{array}{l}\text { Broad well defined high with several elongate } \\
\text { large positive anomalies. Contiguous with IDg01 } \\
\text { and IDg06. Very good correlation with magnetic } \\
\text { anomaly IDm04. }\end{array}$ & $\begin{array}{l}\text { Basaltic lava flows. Located entirely within } \\
\text { topographically defined SRP. }\end{array}$ & Magnetic basaltic rocks. & volcanic mafic & hotspot & $\begin{array}{l}\text { Snake River } \\
\text { Plain }\end{array}$ & $\begin{array}{l}\text { Mabey, 1976; } \\
\text { Sparlin and others, 1982; } \\
\text { Jachens and others, 1989 }\end{array}$ \\
\hline & IDg05 & $\mathbf{H}$ & $\begin{array}{l}\text { NW-trending elongate gravity high (50x5km). } \\
\text { Located mostly over a NW-trending elongate } \\
\text { magnetic low. }\end{array}$ & $\begin{array}{l}\text { Located entirely within SRP over Q } \\
\text { alluvium. }\end{array}$ & $\begin{array}{l}\text { Possibly dense, but weakly or } \\
\text { reversely magnetized intrusive rocks. }\end{array}$ & $\begin{array}{l}\text { intrusive mafic, } \\
\text { intrusive silicic }\end{array}$ & hotspot & $\begin{array}{l}\text { Snake River } \\
\text { Plain }\end{array}$ & \\
\hline $\mathbf{R}$ & IDg06 & $\mathbf{H}$ & $\begin{array}{l}\text { Arcuate regional gravity high, highest to the east. } \\
\text { Contains several distinct smaller anomalies (e.g. } \\
\text { IDg05,07,08). Contiguous with IDg01 and IDg04. } \\
\text { Corresponds with magnetic high IDm10. }\end{array}$ & $\begin{array}{l}\text { Arcuate and well defined topographic } \\
\text { depression. Mostly basaltic lava flows and } \\
\text { cinder cones. }\end{array}$ & $\begin{array}{l}\text { Gravity highs due to dense mafic } \\
\text { intrusive rocks and underplating. }\end{array}$ & $\begin{array}{l}\text { volcanic mafic, } \\
\text { intrusive mafic }\end{array}$ & hotspot & $\begin{array}{l}\text { Snake River } \\
\text { Plain }\end{array}$ & $\begin{array}{l}\text { Mabey, 1976; } \\
\text { Mabey and others, 1978; } \\
\text { Mabey, 1982; } \\
\text { Sparlin and others, 1982; } \\
\text { Jachens and others, 1989 } \\
\end{array}$ \\
\hline 1 & IDg07 & $\mathbf{H}$ & $\begin{array}{l}\text { WNW-trending extremely high gravity terrane } \\
\text { (150x25km). Contiguous with feature IDg08. } \\
\text { Located entirely within larger western and } \\
\text { eastern SRP gravity and magnetic terranes } \\
\text { (IDg06,IDm08). }\end{array}$ & $\begin{array}{l}\text { Located entirely over } \mathrm{Q} \text { and } \mathrm{T} \text { volcanic rocks } \\
\text { of the SRP. }\end{array}$ & $\begin{array}{l}\text { Likely source is dense basaltic lava } \\
\text { flows and associated intrusive rocks. }\end{array}$ & $\begin{array}{l}\text { volcanic mafic, } \\
\text { intrusive mafic }\end{array}$ & hotspot & $\begin{array}{l}\text { Snake River } \\
\text { Plain }\end{array}$ & \\
\hline I & IDg08 & $\mathbf{H}$ & $\begin{array}{l}\text { Irregular shaped, high gravity anomaly with } \\
\text { many small frequency superimposed highs } \\
\text { (125km EWx75km NS). Contiguous with gravity } \\
\text { feature IDg07. Located entirely within larger } \\
\text { western and eastern SRP gravity and magnetic } \\
\text { terranes (IDg06,IDm08). }\end{array}$ & $\begin{array}{l}\text { Located entirely over } \mathrm{Q} \text { and } \mathrm{T} \text { volcanic rocks } \\
\text { of the SRP. }\end{array}$ & $\begin{array}{l}\text { Likely source is dense basaltic lava } \\
\text { flows and associated intrusive rocks. } \\
\text { High frequency superimposed } \\
\text { anomalies may be due to volcanic } \\
\text { vents. }\end{array}$ & $\begin{array}{l}\text { volcanic mafic, } \\
\text { intrusive mafic }\end{array}$ & hotspot & $\begin{array}{l}\text { Snake River } \\
\text { Plain }\end{array}$ & \\
\hline 1 & IDg09 & $\mathbf{H}$ & $\begin{array}{l}\text { NW-trending narrow gravity high (50x10km). } \\
\text { Located at eastern edge of NW-trending } \\
\text { magnetic feature IDm16. }\end{array}$ & $\begin{array}{l}\text { At edge of downwarp of SRP. Located over } \\
Q \text { and T volcanic rocks at the southern edge } \\
\text { of the SRP. }\end{array}$ & $\begin{array}{l}\text { May be volcanic infilling of dense, } \\
\text { magnetic lava flows and possibly } \\
\text { vents forming along tectonic edge of } \\
\text { SRP. }\end{array}$ & $\begin{array}{l}\text { volcanic mafic, } \\
\text { intrusive mafic }\end{array}$ & hotspot & $\begin{array}{l}\text { Snake River } \\
\text { Plain }\end{array}$ & \\
\hline
\end{tabular}


Table 1: Geophysical Features of the Great Basin and parts of the surrounding provinces

\begin{tabular}{|c|c|c|c|c|c|c|c|c|c|}
\hline Scale & ID & $\begin{array}{l}\text { Hi/lo/ } \\
\text { both }\end{array}$ & Terrane definition & Geologic province & Inferred source of anomaly & $\begin{array}{l}\text { Generalized } \\
\text { source rock }\end{array}$ & $\begin{array}{c}\text { Tectonic } \\
\text { setting }\end{array}$ & Province & References \\
\hline I & IDg10 & $\mathbf{H}$ & $\begin{array}{l}\text { EW-trending gravity high (75x25km). } \\
\text { Corresponds with magnetic high IDm17. }\end{array}$ & $\begin{array}{l}\text { At edge of downwarp of SRP. Located over } \\
\mathrm{Q} \text { and T volcanic rocks at the southern edge } \\
\text { of the SRP. }\end{array}$ & $\begin{array}{l}\text { May be volcanic infilling of dense, } \\
\text { magnetic lava flows and possibly } \\
\text { vents forming along tectonic edge of } \\
\text { SRP. }\end{array}$ & $\begin{array}{l}\text { volcanic mafic, } \\
\text { intrusive mafic }\end{array}$ & hotspot & $\begin{array}{l}\text { Snake River } \\
\text { Plain }\end{array}$ & Mabey and others, 1978 \\
\hline \multirow{3}{*}{ I } & IDm01 & $\mathbf{H}$ & $\begin{array}{l}\text { NW-trending magnetic high within SRP } \\
(60 \times 25 \mathrm{~km}) \text {. Located over broad gravity high. }\end{array}$ & $\begin{array}{l}\text { Located over SRP on T and Cretaceous } \\
\text { sediments. Northeast edge borders on T } \\
\text { volcanic rocks. }\end{array}$ & $\begin{array}{l}\text { Likely due to strongly magnetized } \\
\text { intrusive or volcanic rocks. }\end{array}$ & $\begin{array}{l}\text { intrusive mafic, } \\
\text { volcanic mafic }\end{array}$ & hotspot & $\begin{array}{l}\text { Snake River } \\
\text { Plain }\end{array}$ & \\
\hline & IDm02 & $\mathbf{H}$ & Irregular magnetic high $(60 \times 30 \mathrm{~km})$. & $\begin{array}{l}\text { Located over T intrusive on western edge of } \\
\text { Idaho Batholith. }\end{array}$ & Magnetic intrusive rocks. & intrusive mafic & uplift & $\begin{array}{l}\text { Northern } \\
\text { Rocky Mtns }\end{array}$ & Mabey, 1986 \\
\hline & IDm03 & $\mathbf{H}$ & $\begin{array}{l}\text { NW-trending prominent magnetic high within } \\
\text { the SRP (75x25km). }\end{array}$ & $\begin{array}{l}\text { Located within SRP over Q sediments and } \\
\text { some Q and T volcanic rocks. Anomaly is } \\
\text { parallel to several NW-trending features } \\
\text { crossing the eastern SRP. }\end{array}$ & Magnetic intrusive or volcanic rocks. & $\begin{array}{l}\text { intrusive mafic, } \\
\text { volcanic mafic }\end{array}$ & hotspot & $\begin{array}{l}\text { Snake River } \\
\text { Plain }\end{array}$ & \\
\hline \multirow[t]{4}{*}{ I } & IDm04 & $\mathbf{H}$ & $\begin{array}{l}\text { Well defined region of small to intermediate } \\
\text { sized (50-75km) anomalies. Regionally a high } \\
\text { magnetic area. Very good correlation with } \\
\text { gravity anomaly IDg04. Contiguous with IDm } 08 \\
\text { and IDm10. }\end{array}$ & $\begin{array}{l}\text { Basaltic lava flows. Located entirely within } \\
\text { topographically defined SRP. }\end{array}$ & Dense basaltic rocks. & volcanic mafic & hotspot & $\begin{array}{l}\text { Snake River } \\
\text { Plain }\end{array}$ & $\begin{array}{l}\text { Sparlin and others, 1982; } \\
\text { Mabey, 1986; } \\
\text { Jachens and others, } 1989\end{array}$ \\
\hline & IDm05 & $\mathbf{H}$ & $\begin{array}{l}\text { NW-trending elongate magnetic high within the } \\
\text { western SRP (30x15km). }\end{array}$ & $\begin{array}{l}\text { Located within western SRP over } \mathrm{Q} \text { volcanic } \\
\text { rocks. }\end{array}$ & $\begin{array}{l}\text { Magnetic volcanic or buried intrusive } \\
\text { rocks. }\end{array}$ & $\begin{array}{l}\text { intrusive mafic, } \\
\text { volcanic mafic }\end{array}$ & hotspot & $\begin{array}{l}\text { Snake River } \\
\text { Plain }\end{array}$ & \\
\hline & IDm06 & $\mathbf{H}$ & $\begin{array}{l}\text { EW-trending string of local magnetic highs } \\
(75 \times 25 \mathrm{~km}) \text {. }\end{array}$ & $\begin{array}{l}\text { Located at south end of Idaho Batholith over } \\
\text { Mz intrusive and T volcanic rocks. }\end{array}$ & $\begin{array}{l}\text { Magnetic volcanic or shallow } \\
\text { intrusive rocks. }\end{array}$ & $\begin{array}{l}\text { intrusive mafic, } \\
\text { volcanic mafic }\end{array}$ & compression & $\begin{array}{l}\text { Northern } \\
\text { Rocky Mtns }\end{array}$ & \\
\hline & IDm07 & $\mathbf{H}$ & $\begin{array}{l}\text { NW-trending elongate string of magnetic highs } \\
(125 \times 25 \mathrm{~km}) \text {. }\end{array}$ & $\begin{array}{l}\text { Crosses at right angle to the northern edge } \\
\text { of SRP and spans Cz volcanic, Cz and Mz } \\
\text { intrusive, and Pz clastic rocks. Parallels } \\
\text { trend of basins and ranges north of the SRP. } \\
\text { Follows the NW-trending Craters of the } \\
\text { Moon rift. }\end{array}$ & $\begin{array}{l}\text { Magnetic intrusive and volcanic } \\
\text { rocks. }\end{array}$ & $\begin{array}{l}\text { intrusive mafic, } \\
\text { volcanic mafic }\end{array}$ & $\begin{array}{l}\text { hotspot, } \\
\text { compression }\end{array}$ & $\begin{array}{l}\text { Northern } \\
\text { Rocky Mtns, } \\
\text { Snake River } \\
\text { Plain }\end{array}$ & Mabey and others, 1978 \\
\hline \multirow[t]{2}{*}{ I } & IDm08 & $\mathbf{H}$ & $\begin{array}{l}\text { NW-trending region consisting of several high } \\
\text { frequency and some prominent long linear } \\
\text { anomalies (up to 200km). Magnetic terrane is } \\
\text { south of and narrower than the gravity terrane } \\
\text { (IDg01). Contiguous with IDm04 and IDm10. }\end{array}$ & $\begin{array}{l}\text { Veneer of alluvium overlying basaltic lava } \\
\text { flows. }\end{array}$ & Dense and magnetic basaltic rocks. & volcanic mafic & hotspot & $\begin{array}{l}\text { Snake River } \\
\text { Plain }\end{array}$ & $\begin{array}{l}\text { Sparlin and others, 1982; } \\
\text { Mabey, 1982; } \\
\text { Jachens and others, } 1989\end{array}$ \\
\hline & IDm09 & $\mathbf{H}$ & $\begin{array}{l}\text { NW-trending prominent magnetic high within } \\
\text { the eastern SRP }(30 \times 15 \mathrm{~km}) \text {. }\end{array}$ & $\begin{array}{l}\text { Located within eastern SRP over Q volcanic } \\
\text { rocks. }\end{array}$ & $\begin{array}{l}\text { Magnetic volcanic rocks or buried } \\
\text { intrusive. }\end{array}$ & $\begin{array}{l}\text { volcanic mafic, } \\
\text { intrusive mafic }\end{array}$ & hotspot & $\begin{array}{l}\text { Snake River } \\
\text { Plain }\end{array}$ & \\
\hline \multirow[t]{4}{*}{$\mathbf{R}$} & IDm10 & $\mathbf{H}$ & $\begin{array}{l}\text { Arcuate regional magnetic high consisting of } \\
\text { small to intermediate sized anomalies (<100km) } \\
\text { in most places displaying a NW-fabric (e.g. } \\
\text { IDm03,07,09). Corresponds with gravity high } \\
\text { IDg06. Contiguous with IDm04 and IDm08. } \\
\end{array}$ & $\begin{array}{l}\text { Arcuate and well defined topographic } \\
\text { depression. Mostly basaltic lava flows and } \\
\text { cinder cones. }\end{array}$ & $\begin{array}{l}\text { Magnetic mafic volcanic and intrusive } \\
\text { rocks. }\end{array}$ & $\begin{array}{l}\text { volcanic mafic, } \\
\text { intrusive mafic }\end{array}$ & hotspot & $\begin{array}{l}\text { Snake River } \\
\text { Plain }\end{array}$ & $\begin{array}{l}\text { Mabey, 1976; } \\
\text { Mabey and others, 1978; } \\
\text { Mabey, 1982; } \\
\text { Sparlin and others, 1982; } \\
\text { Jachens and others, } 1989 \\
\end{array}$ \\
\hline & IDm11 & $\mathrm{L}$ & $\begin{array}{l}\text { Large, pronounced magnetic low }(250 \times 125 \mathrm{~km}) \\
\text { that corresponds with gravity high extending off } \\
\text { of the eastern SRP. }\end{array}$ & $\begin{array}{l}\text { Located over thrusted strata of the Rocky } \\
\text { Mtns thrust belt. }\end{array}$ & $\begin{array}{l}\text { Weakly magnetic, high density } \\
\text { carbonate rocks. }\end{array}$ & $\begin{array}{l}\text { sedimentary } \\
\text { carbonate }\end{array}$ & compression & $\begin{array}{l}\text { Great Basin, } \\
\text { Middle Rocky } \\
\text { Mtns }\end{array}$ & \\
\hline & IDm12 & $\mathbf{L}$ & $\begin{array}{l}\text { Prominent magnetic low }(100 \times 50 \mathrm{~km}) \text { located over } \\
\text { a moderate gravity low of the same general size. }\end{array}$ & $\begin{array}{l}\text { Located over Mz intrusive rocks. May be } \\
\text { related to Idaho Batholith. }\end{array}$ & $\begin{array}{l}\text { Weakly magnetic and relatively low } \\
\text { density intrusive rocks. }\end{array}$ & intrusive silicic & batholith & Great Basin & \\
\hline & IDm13 & H & $\begin{array}{l}\text { EW-trending prominent magnetic high within } \\
\text { the eastern SRP }(30 \times 15 \mathrm{~km}) \text {. }\end{array}$ & $\begin{array}{l}\text { Located within eastern SRP over Q volcanic } \\
\text { rocks. }\end{array}$ & $\begin{array}{l}\text { Magnetic volcanic or buried intrusive } \\
\text { rocks. }\end{array}$ & $\begin{array}{l}\text { volcanic mafic, } \\
\text { intrusive mafic }\end{array}$ & hotspot & $\begin{array}{l}\text { Snake River } \\
\text { Plain }\end{array}$ & \\
\hline
\end{tabular}




\begin{tabular}{|c|c|c|c|c|c|c|c|c|c|}
\hline Scale & ID & $\begin{array}{l}\mathrm{Hi} / \mathrm{lo} / \\
\text { both }\end{array}$ & Terrane definition & Geologic province & Inferred source of anomaly & $\begin{array}{l}\text { Generalized } \\
\text { source rock }\end{array}$ & $\begin{array}{c}\text { Tectonic } \\
\text { setting }\end{array}$ & Province & References \\
\hline 1 & IDm14 & $\mathbf{H}$ & $\begin{array}{l}\text { NW-trending subdued magnetic high } \\
(100 \times 30 \mathrm{~km}) \text { branching off of eastern SRP. } \\
\text { Bounds Magnetic low domain to northeast. }\end{array}$ & $\begin{array}{l}\text { Located over early Pz stratified rocks, pC } \\
\text { metamorphic, and T sedimentary rocks. } \\
\text { Parallels trend of thrust slices of Pz strata. } \\
\text { Cuts across and is normal to the southern } \\
\text { edge of SRP. Has similar trend to hotspot- } \\
\text { related rift features such as the Great Rift } \\
\text { and to narrow NW-trending features in } \\
\text { Utah (UTg02, UTm02). }\end{array}$ & $\begin{array}{l}\text { May be similar in origin to feature } \\
\text { UTm02 - rift related mafic magnetic } \\
\text { intrusive or volcanic rocks or } \\
\text { Precambrian basement. }\end{array}$ & $\begin{array}{l}\text { intrusive } \\
\text { mafic, volcanic } \\
\text { mafic, basement } \\
\text { mafic }\end{array}$ & hotspot & $\begin{array}{l}\text { Great Basin, } \\
\text { Snake River } \\
\text { Plain }\end{array}$ & Mabey and others, 1978 \\
\hline I & IDm15 & $\mathbf{H}$ & $\begin{array}{l}\text { Irregular magnetic terrain similar in character } \\
\text { with SRP. Forms a tongue extending off western } \\
\text { SRP in line and parallel to eastern SRP. Includes } \\
\text { a prominent U-shaped magnetic high at its SW } \\
\text { end (IDm18). Possibly a fossil trace of } \\
\text { Yellowstone hotspot. Forms part of the } \\
\text { Humboldt magnetic zone of Mabey and others } \\
\text { (1978). }\end{array}$ & Located over $\mathrm{T}$ and $\mathrm{Q}$ volcanic rocks. & $\begin{array}{l}\text { Magnetic volcanic and intrusive } \\
\text { rocks. }\end{array}$ & $\begin{array}{l}\text { volcanic mafic, } \\
\text { intrusive mafic }\end{array}$ & hotspot & $\begin{array}{l}\text { Snake River } \\
\text { Plain }\end{array}$ & $\begin{array}{l}\text { Mabey and others, 1978; } \\
\text { Hinze and Zietz, 1985 }\end{array}$ \\
\hline I & IDm16 & $\mathbf{H}$ & $\begin{array}{l}\text { NW-trending narrow magnetic high (190x40km). } \\
\text { Includes gravity IDg09, though largely located } \\
\text { outside the gravity defined western SRP over } \\
\text { regional gravity high of northern GB (NVg04). } \\
\text { Northern end converges on the southern edge of } \\
\text { the topographic and gravity defined SRP. } \\
\text { Feature narrows to the NW. }\end{array}$ & $\begin{array}{l}\text { Northern } 2 / 3 \text { of anomaly parallels western } \\
\text { SRP. Southern end extends over northern } \\
\text { GB. }\end{array}$ & $\begin{array}{l}\text { Probably due to extensive magnetic } \\
\text { volcanic and intrusive rocks. }\end{array}$ & $\begin{array}{l}\text { volcanic mafic, } \\
\text { intrusive mafic }\end{array}$ & hotspot & $\begin{array}{l}\text { Snake River } \\
\text { Plain, Great } \\
\text { Basin }\end{array}$ & Mabey and others, 1978 \\
\hline \multirow[t]{2}{*}{ I } & IDm17 & $\mathbf{H}$ & $\begin{array}{l}\text { EW-trending magnetic high (75x10km). } \\
\text { Corresponds with gravity high IDg10. }\end{array}$ & $\begin{array}{l}\text { At edge of downwarp of SRP. Located over } \\
Q \text { and T volcanic rocks at the southern edge } \\
\text { of the SRP. }\end{array}$ & $\begin{array}{l}\text { May be volcanic infilling of dense, } \\
\text { magnetic lava flows and possibly } \\
\text { vents forming along tectonic edge of } \\
\text { SRP. }\end{array}$ & $\begin{array}{l}\text { volcanic mafic, } \\
\text { intrusive mafic }\end{array}$ & hotspot & $\begin{array}{l}\text { Snake River } \\
\text { Plain }\end{array}$ & Mabey and others, 1978 \\
\hline & IDm18 & $\mathbf{H}$ & $\begin{array}{l}\text { U-shaped magnetic high }(75 \times 20 \mathrm{~km}) \text { that is } \\
\text { located over a gravity high. }\end{array}$ & Located over T lava flows. & $\begin{array}{l}\text { Magnetic volcanic and intrusive } \\
\text { rocks. }\end{array}$ & $\begin{array}{l}\text { intrusive mafic, } \\
\text { volcanic mafic }\end{array}$ & $\begin{array}{l}\text { hotspot, } \\
\text { extension }\end{array}$ & Great Basin & \\
\hline \multirow[t]{2}{*}{1} & NVg01 & B & $\begin{array}{l}\text { NE-trending boundary between high gravity to } \\
\text { SE and low gravity to NW. Very well correlated } \\
\text { with magnetic boundary. Corresponds with } \\
\text { magnetic feature NVm05. }\end{array}$ & $\begin{array}{l}\text { West of this line there are no pre-Cz rocks } \\
\text { exposed. The boundary also corresponds } \\
\text { with the eastern edge of topographic and } \\
\text { basaltic plateau. Note that this boundary } \\
\text { corresponds with the edge of basement } \\
\text { gravity calculation. See Isostatic gravity } \\
\text { map. }\end{array}$ & $\begin{array}{l}\text { The prominent gravity anomaly likely } \\
\text { reflects a major deep crustal } \\
\text { discontinuity. }\end{array}$ & $\begin{array}{l}\text { volcanic mafic, } \\
\text { sedimentary } \\
\text { siliceous }\end{array}$ & $\begin{array}{l}\text { transcurrent, } \\
\text { accretion }\end{array}$ & Great Basin & $\begin{array}{l}\text { McKee and others, 1983; } \\
\text { Blakely, 1988; } \\
\text { McKee and others, 1990; } \\
\text { Wyld and Wright, 2001 }\end{array}$ \\
\hline & NVg02 & $\mathbf{H}$ & $\begin{array}{l}\text { ENE-trending gravity high mainly defined in } \\
\text { NW Nevada }(225 \times 100 \mathrm{~km}) \text {. }\end{array}$ & $\begin{array}{l}\text { Located mostly over Mz metaclastic rocks } \\
\text { (including mudstones and sandstones). } \\
\text { Crosses several pre-Cz lithologic terranes } \\
\text { and the continental margin inferred from Sr- } \\
\text { isotopic ratios. Anomaly lies in line with } \\
\text { eastern SRP and is coincident with a region } \\
\text { of high heatflow extending SW from the } \\
\text { eastern SRP. Anomaly is not easily } \\
\text { distinguished from general gravity high } \\
\text { south of the SRP. Northern edge of anomaly } \\
\text { is poorly defined. }\end{array}$ & $\begin{array}{l}\text { May reflect a region of uplifted dense } \\
\text { lower crustal rocks. }\end{array}$ & basement mafic & uplift & Great Basin & \\
\hline $\mathbf{I}$ & NVg03 & $\mathrm{L}$ & $\begin{array}{l}\text { Oval gravity low }(50 \mathrm{~km}) \text { within broad regional } \\
\text { gravity high of northern GB (NVg04). }\end{array}$ & $\begin{array}{l}\text { Located over western assemblage (upper } \\
\text { plate of Roberts Mt thrust) cherts and shales } \\
\text { at north end of Carlin trend. Small plutons } \\
\text { crop out at center of anomaly. }\end{array}$ & May be due to a large buried pluton. & intrusive silicic & extension & Great Basin & \\
\hline
\end{tabular}




\begin{tabular}{|c|c|c|c|c|c|c|c|c|c|}
\hline Scale & ID & $\begin{array}{l}\mathrm{H} \text { Hi/lo/ } \\
\text { both }\end{array}$ & Terrane definition & Geologic province & Inferred source of anomaly & $\begin{array}{l}\text { Generalized } \\
\text { source rock }\end{array}$ & $\begin{array}{c}\text { Tectonic } \\
\text { setting }\end{array}$ & Province & References \\
\hline \multirow[t]{2}{*}{$\mathbf{R}$} & NVg04 & $\mathbf{H}$ & $\begin{array}{l}\text { Regional gravity high across northern GB. } \\
\text { Extends from the SRP southward. Southern } \\
\text { boundary (at } 40^{\circ} \text { latitude) is marked by an abrupt } \\
\text { gradient to low regional gravity of the southern } \\
\text { GB regional gravity low (NVg10). Anomaly } \\
\text { largely coincides with a regional magnetic low } \\
\text { (UTm01). }\end{array}$ & $\begin{array}{l}\text { The central and eastern part of the anomaly } \\
\text { is underlain largely by Pz carbonate rocks. } \\
\text { The western and northern parts are } \\
\text { coincident with Pz silicic sedimentary rocks } \\
\text { overlain by Miocene volcanic rocks that } \\
\text { extend north into OR, ID, and SRP. }\end{array}$ & $\begin{array}{l}\text { Underlain by low magnetic, high } \\
\text { density (likely carbonate rocks). } \\
\text { Anomaly may indicate that carbonate } \\
\text { rocks extend to the SRP and that } \\
\text { volcanic rocks form only a thin veneer } \\
\text { (since magnetic anomaly is low). } \\
\text { Alternatively, gravity high may } \\
\text { reflect dense mafic underplating } \\
\text { associated with the Yellowstone } \\
\text { hotspot. }\end{array}$ & $\begin{array}{l}\text { sedimentary } \\
\text { carbonate, } \\
\text { intrusive mafic }\end{array}$ & $\begin{array}{l}\text { continental } \\
\text { margin, } \\
\text { hotspot }\end{array}$ & Great Basin & $\begin{array}{l}\text { Glen and others, 2000; } \\
\text { Pierce and others, 2000 }\end{array}$ \\
\hline & NVg05 & $\mathbf{H}$ & $\begin{array}{l}\text { ENE-trending gravity high (80x25km). } \\
\text { Corresponds with a magnetic low. }\end{array}$ & $\begin{array}{l}\text { Located over T volcanic, Pz and Mz } \\
\text { sedimentary, and Mz intrusive rocks near } \\
\text { the boundary of the Black Rock and Jungo } \\
\text { lithologic terranes. }\end{array}$ & $\begin{array}{l}\text { May be due to dense intrusive or } \mathrm{Mz} \\
\text { metasedimentary rocks. }\end{array}$ & basement mafic & $\begin{array}{l}\text { subduction, } \\
\text { continental } \\
\text { margin }\end{array}$ & Great Basin & \\
\hline 1 & NVg06 & $\mathbf{H}$ & $\begin{array}{l}\text { Regional gravity high. Generally associated with } \\
\text { magnetic low, but also spans magnetic highs of } \\
\text { western and central NNR. }\end{array}$ & $\begin{array}{l}\text { Located over T volcanic, Mz sedimentary } \\
\text { and intrusive, and Pz sedimentary rocks. } \\
\text { Centered on Jurassic Humboldt lopolith } \\
\text { gabbro and basalts. }\end{array}$ & $\begin{array}{l}\text { May be due to dense mafic intrusive } \\
\text { rocks including the Jurassic } \\
\text { Humboldt lopolith. }\end{array}$ & intrusive mafic & $\begin{array}{l}\text { continental } \\
\text { margin }\end{array}$ & Great Basin & $\begin{array}{l}\text { Page, 1965; } \\
\text { Speed, 1976; } \\
\text { Oldow, 1984; } \\
\text { Dilek and Moores, 1995; } \\
\text { Hildenbrand and others, } 2000 \\
\end{array}$ \\
\hline \multirow[t]{2}{*}{ I } & NVg07 & $\mathbf{H}$ & $\begin{array}{l}\text { Prominent gravity high over north central } \\
\text { Nevada. Southern boundary of the high has a } \\
\text { distinct 'V' shape. Anomaly is part of the } \\
\text { Northern GB gravity high terrane (NVg0). West } \\
\text { edge of gravity 'V' corresponds closely with the } \\
\text { Northern Nevada Rift (NVm12). }\end{array}$ & $\begin{array}{l}\text { East edge of gravity ' } \mathrm{V} \text { ' corresponds with } \\
\text { middle Pz Roberts Mt thrust front. West } \\
\text { edge may reflect an older crustal } \\
\text { discontinuity. }\end{array}$ & $\begin{array}{l}\text { Gravity high (inside 'V' relates to } \\
\text { regional high of northern GB - } \\
\text { NVg04). East edge may reflect crustal } \\
\text { discontinuity at thrust juxtaposing } \\
\text { dense Pz sedimentary rocks to the } \\
\text { NW with less dense sedimentary } \\
\text { rocks to the SE. West edge may } \\
\text { reflect an older crustal discontinuity } \\
\text { associated with the pC continental } \\
\text { margin. }\end{array}$ & $\begin{array}{l}\text { sedimentary } \\
\text { siliceous, } \\
\text { basement mafic }\end{array}$ & $\begin{array}{l}\text { continental } \\
\text { margin, } \\
\text { accretion }\end{array}$ & Great Basin & $\begin{array}{l}\text { Grauch and others, 1995; } \\
\text { Hildenbrand and others, 2000; } \\
\text { Ponce and Glen, } 2002\end{array}$ \\
\hline & NVg08 & $\mathbf{H}$ & $\begin{array}{l}\text { Gravity high }(125 \times 60 \mathrm{~km}) \text {. Corresponds with } \\
\text { west end of feature NVm14 associated with } \\
\text { diverse magnetic signature of the Walker Lane } \\
\text { belt. }\end{array}$ & $\begin{array}{l}\text { Located largely over the Carson Sink and } \\
\text { over T volcanic rocks on the west edge of } \\
\text { anomaly. Also located over some Mz } \\
\text { intrusive rocks. }\end{array}$ & $\begin{array}{l}\text { May be due to dense mafic to } \\
\text { intermediate composition volcanic } \\
\text { and associated intrusive rocks. }\end{array}$ & $\begin{array}{l}\text { volcanic mafic, } \\
\text { intrusive mafic }\end{array}$ & subduction & Great Basin & Hildenbrand and others, 2000 \\
\hline I & NVg09 & $\mathbf{L}$ & $\begin{array}{l}\text { NW-trending gravity low bounded to SW by the } \\
\text { generally high gravity over the Walker Lane belt } \\
\text { (NVg11). Located over a regional magnetic low } \\
\text { punctuated by highs of NNR anomalies. } \\
\text { Bounded on the northeast by the gravity high of } \\
\text { the Northern Nevada basement gravity V } \\
\text { (NVg07). At its eastern edge it merges with } \\
\text { feature NVg10. }\end{array}$ & $\begin{array}{l}\text { Located over intermediate composition tuffs } \\
\text { that lie on Mz strata in the west, and on Pz } \\
\text { strata in the east. }\end{array}$ & $\begin{array}{l}\text { Low density tuffs, and quartzose } \\
\text { sedimentary rocks. }\end{array}$ & $\begin{array}{l}\text { sedimentary } \\
\text { silicic }\end{array}$ & subduction & Great Basin & \\
\hline $\mathbf{R}$ & NVg10 & $\mathrm{L}$ & $\begin{array}{l}\text { Regional gravity low that extends from } \sim 37^{\circ} \\
\text { latitude in the south to } \sim 40^{\circ} \text { latitude in the north. } \\
\text { Anomaly located mostly over regional magnetic } \\
\text { low (UTm01). On its western edge, anomaly } \\
\text { merges with feature NVm09. }\end{array}$ & $\begin{array}{l}\text { Western part is located mostly over T } \\
\text { volcanic and Pz sedimentary rocks. To the } \\
\text { east, anomaly located mostly over Pz rocks. } \\
\text { Pz rocks on the west are more silicic than } \\
\text { those to the east. Region lacks significant } \\
\text { basalt outcrops such as occur to the north. }\end{array}$ & \begin{tabular}{|l|} 
Anomaly due to low density and low \\
magnetic source rocks, which may \\
indicate that Pz rocks are thinner \\
here than to the north beneath feature \\
NVg04.
\end{tabular} & $\begin{array}{l}\text { sedimentary } \\
\text { silicic }\end{array}$ & extension & Great Basin & $\begin{array}{l}\text { Blakely and Jachens, 1991; } \\
\text { Glen and others, } 2000\end{array}$ \\
\hline 1 & NVg11 & $\mathbf{H}$ & $\begin{array}{l}\text { NW-trending elongate zone }(\sim 100 \mathrm{~km} \times 350 \mathrm{~km}) \text { in } \\
\text { western NV, regional positive anomaly with } \\
\text { several prominent local }(\sim 25 \mathrm{~km}) \text { positive } \\
\text { anomalies. Located within diverse magnetic } \\
\text { feature NVm23. }\end{array}$ & $\begin{array}{l}\text { Structurally defined province characterized } \\
\text { by NW-trending topographic features. } \\
\text { located over Mz granites, Pz strata, and late } \\
\text { T volcanic rocks. Corresponds mostly with } \\
\text { the physiographically defined Walker Lane } \\
\text { belt. }\end{array}$ & $\begin{array}{l}\text { Gravity feature is possibly due to T } \\
\text { mafic underplating. Magnetic } \\
\text { anomalies may express shallow to } \\
\text { deeply buried magnetic plutons . } \\
\text { Possibly represents the eastern edge } \\
\text { of Sierra Nevada Batholith. }\end{array}$ & intrusive mafic & $\begin{array}{l}\text { transcurrent, } \\
\text { extension, } \\
\text { continental } \\
\text { margin }\end{array}$ & Great Basin & $\begin{array}{l}\text { Blakely, 1988; } \\
\text { Craig, 1992; } \\
\text { Hildenbrand and others, } 2000\end{array}$ \\
\hline
\end{tabular}


Table 1: Geophysical Features of the Great Basin and parts of the surrounding provinces

\begin{tabular}{|c|c|c|c|c|c|c|c|c|c|}
\hline Scale & ID & $\begin{array}{l}\mathrm{H} i / \mathrm{lo} / \\
\text { both }\end{array}$ & Terrane definition & Geologic province & Inferred source of anomaly & $\begin{array}{l}\text { Generalized } \\
\text { source rock }\end{array}$ & $\begin{array}{c}\text { Tectonic } \\
\text { setting }\end{array}$ & Province & References \\
\hline I & NVg12 & $\mathbf{H}$ & $\begin{array}{l}\text { Roughly oval ( } 125 \mathrm{~km} \text { EWx100km NS) diffuse } \\
\text { gravity high. Located over a regional magnetic } \\
\text { low. Probably relates to feature NVg16. }\end{array}$ & $\begin{array}{l}\text { Located over diverse range of rock types } \\
\text { including T volcanic and Pz sedimentary } \\
\text { rocks. }\end{array}$ & $\begin{array}{l}\text { Likely source is thick accumulation of } \\
\text { limestones and dolomites. }\end{array}$ & $\begin{array}{l}\text { sedimentary } \\
\text { carbonate }\end{array}$ & $\begin{array}{l}\text { continental } \\
\text { margin }\end{array}$ & Great Basin & \\
\hline I & NVg13 & L & Oval gravity low (50km). & $\begin{array}{l}\text { Located over alluvial basin west of Wheeler } \\
\text { Peak. NE end of anomaly located over } \\
\text { granitic plutons. }\end{array}$ & $\begin{array}{l}\text { May be due to thick pile of low } \\
\text { density alluvial fill, and/or low } \\
\text { density granitic plutons. }\end{array}$ & $\begin{array}{l}\text { sedimentary } \\
\text { siliceous, } \\
\text { intrusive silicic }\end{array}$ & extension & Great Basin & \\
\hline \multirow[t]{2}{*}{1} & NVg14 & $\mathbf{L}$ & $\begin{array}{l}\text { Prominent NW-trending gravity low }(120 \times 30 \mathrm{~km}) \\
\text { located over a magnetic low. }\end{array}$ & $\begin{array}{l}\text { Corresponds with the Jurassic Sylvania } \\
\text { pluton in the Sylvania Mtns. }\end{array}$ & Low density non-magnetic pluton. & intrusive silicic & batholith & Great Basin & McKee, 1968 \\
\hline & NVg15 & $\mathbf{H}$ & $\begin{array}{l}\text { NS-trending gravity high }(60 \times 40 \mathrm{~km}) \text {. } \\
\text { Coresponds with intense parts of magnetic } \\
\text { feature NVm19. }\end{array}$ & $\begin{array}{l}\text { Located largely over T volcanic rocks. Some } \\
\text { outcrops of Mz sedimentary and T intrusive } \\
\text { rocks at the south end of anomaly. }\end{array}$ & $\begin{array}{l}\text { May be due to dense volcanic and } \\
\text { associated intrusive rocks. }\end{array}$ & $\begin{array}{l}\text { volcanic mafic, } \\
\text { intrusive mafic }\end{array}$ & batholith & Great Basin & \\
\hline I & NVg16 & $\mathbf{H}$ & $\begin{array}{l}\text { EW-trending variable gravity high }(200 \times 50 \mathrm{~km}) \text {. } \\
\text { Located over a regional magnetic low Probably } \\
\text { relates to feature NVg12. }\end{array}$ & $\begin{array}{l}\text { Located over T volcanic and Pz carbonate } \\
\text { rocks, though largely follows the along } \\
\text { northern and western edge of an extensive } \\
\text { outcrop of Pz carbonates. }\end{array}$ & $\begin{array}{l}\text { Dense, weakly magnetic carbonate } \\
\text { rocks. }\end{array}$ & $\begin{array}{l}\text { sedimentary } \\
\text { carbonate }\end{array}$ & $\begin{array}{l}\text { continental } \\
\text { margin }\end{array}$ & Great Basin & \\
\hline I & NVg17 & $\mathrm{L}$ & $\begin{array}{l}\text { NW-trending gravity low }(30 \times 20 \mathrm{~km}) \text {. } \\
\text { Corresponds with magnetic anomaly NVm21. }\end{array}$ & Wilson Creek Mtns - late Oligocene caldera. & $\begin{array}{l}\text { Thick accumulation of low density, } \\
\text { magnetic caldera fill tuffs and } \\
\text { underlying silicic intrusive rocks. }\end{array}$ & volcanic silicic & subduction & Great Basin & $\begin{array}{l}\text { Best and others, 1989a; } \\
\text { Best and others, 1989b }\end{array}$ \\
\hline I & NVg18 & B & $\begin{array}{l}\text { Boundary between low basement gravity to the } \\
\text { north and higher basement gravity to the south. } \\
\text { Also an alignment of several prominent } \\
\text { anomalies across Sierra Nevada, southern GB, } \\
\text { and CP. No or little magnetic expression. }\end{array}$ & $\begin{array}{l}\text { Southern end of GB. Corresponds with } \\
\text { topographic drop from north to south, and } \\
\text { to a band of seismicity rimming the Basin } \\
\text { and Range. }\end{array}$ & $\begin{array}{l}\text { Speculated (Saltus and Thompson, } \\
\text { 1995) to relate to a combination of } \\
\text { thermal buoyancy (related to } \\
\text { Yellowstone plume head), thickened } \\
\text { crust and mid-lower crustal felsic and } \\
\text { mafic dikes and sill injection beneath } \\
\text { the gravity high region. }\end{array}$ & $\begin{array}{l}\text { intrusive mafic, } \\
\text { intrusive felsic }\end{array}$ & $\begin{array}{l}\text { subduction, } \\
\text { hotspot }\end{array}$ & Great Basin & $\begin{array}{l}\text { Stewart and others, 1977; } \\
\text { Eaton and others, 1978; } \\
\text { Saltus and Thompson 1995; } \\
\text { Pierce and others, } 2000\end{array}$ \\
\hline I & NVg20 & $\mathbf{L}$ & $\begin{array}{l}\text { Oval east-trending gravity low }(75 \times 40 \mathrm{~km}) \text {. Partly } \\
\text { coincident with anomaly NVm24. }\end{array}$ & $\begin{array}{l}\text { West end of Clover Mtns and part of } \\
\text { Caliente depression and Caliente and Kane } \\
\text { Springs Wash calderas. Also over Pz } \\
\text { sedimentary rocks. }\end{array}$ & $\begin{array}{l}\text { Thick accumulation of low density } \\
\text { rhyolitic and tuffaceous sedimentary } \\
\text { rocks. }\end{array}$ & volcanic silicic & subduction & Great Basin & $\begin{array}{l}\text { Noble and McKee, 1972; } \\
\text { Best and others, 1989b }\end{array}$ \\
\hline I & NVg21 & $\mathbf{H}$ & $\begin{array}{l}\text { Irregular shaped gravity high. Generally NW- } \\
\text { trending (max 300km NWx125km SE). }\end{array}$ & $\begin{array}{l}\text { Located over pC crystalline, Pz carbonate } \\
\text { and quartzite, and T volcanic rocks. } \\
\text { Includes most of Death Valley and Pahrump } \\
\text { Valley. }\end{array}$ & $\begin{array}{l}\text { Likely source is pC crystalline } \\
\text { basement and thick accumulation of } \\
\text { limestones and dolomites. }\end{array}$ & basement silicic & $\begin{array}{l}\text { extension, } \\
\text { uplift }\end{array}$ & Great Basin & \\
\hline L & NVg22 & $\mathrm{H}$ & $\begin{array}{l}\text { Small oval gravity high }(10 \mathrm{~km}) \text {. Corresponds } \\
\text { partly with magnetic high NVm30. }\end{array}$ & Fault block of $\mathrm{pC}$ to $\mathrm{Mz}$ sedimentary rocks. & $\begin{array}{l}\text { Speculated (Blank, 1988; Blakely, } \\
\text { 1988) to be due to upwarp in dense } \\
\text { and magnetic crystalline basement } \\
\text { and to an associated Tintrusion. }\end{array}$ & basement mafic & uplift & Great Basin & $\begin{array}{l}\text { Stewart and Carlson, 1978; } \\
\text { Blank, 1988; } \\
\text { Blakely, } 1988\end{array}$ \\
\hline I & NVg23 & $\mathrm{H}$ & $\begin{array}{l}\text { Irregular shaped gravity high }(140 \times 125 \mathrm{~km}) \text {. Bulk } \\
\text { of anomaly located over magnetic low. Entire } \\
\text { anomaly crosses numerous local magnetic } \\
\text { anomalies. }\end{array}$ & \begin{tabular}{|l|} 
Located largely over pC rocks of the Virgin \\
Valley.
\end{tabular} & Dense pC crystalline rocks. & basement silicic & $\begin{array}{l}\text { extension, } \\
\text { uplift }\end{array}$ & Great Basin & Langenheim and others, 2000 \\
\hline \multirow{3}{*}{ L } & NVm01 & $\mathbf{H}$ & $\begin{array}{l}\text { Elongate north-trending magnetic high } \\
(100 \times 25 \mathrm{~km}) .\end{array}$ & $\begin{array}{l}\text { Located over T volcanic and sedimentary } \\
\text { rocks. Has similar trend as the western } \\
\text { NNR. }\end{array}$ & $\begin{array}{l}\text { Strongly magnetic volcanic rocks or } \\
\text { mafic intrusive rocks. }\end{array}$ & $\begin{array}{l}\text { intrusive mafic, } \\
\text { volcanic mafic }\end{array}$ & $\begin{array}{l}\text { extension, } \\
\text { hotspot }\end{array}$ & Great Basin & $\begin{array}{l}\text { Glen and Ponce, 2002; } \\
\text { Ponce and Glen, 2002 }\end{array}$ \\
\hline & NVm02 & $\mathbf{H}$ & $\begin{array}{l}\text { Oval cluster of high frequency positive magnetic } \\
\text { anomalies }(25 \mathrm{~km}) \text {. }\end{array}$ & McDermitt group of caldera. & $\begin{array}{l}\text { Magnetic intrusive and extrusive } \\
\text { rocks. }\end{array}$ & $\begin{array}{l}\text { intrusive mafic, } \\
\text { volcanic silicic }\end{array}$ & $\begin{array}{l}\text { extension, } \\
\text { hotspot }\end{array}$ & Great Basin & $\begin{array}{l}\text { U.S. Geological Survey 1972; } \\
\text { U.S. Geological Survey 1982a, b; } \\
\text { Rytuba and McKee, 1984; } \\
\text { Best and others, 1989b; }\end{array}$ \\
\hline & NVm03 & $\mathbf{H}$ & $\begin{array}{l}\text { Elongate NW-trending moderate magnetic high } \\
(50 \times 20 \mathrm{~km}) \text {. }\end{array}$ & Located over T volcanic rocks. & Strongly magnetic volcanic rocks. & $\begin{array}{l}\text { volcanic mafic, } \\
\text { intrusive mafic }\end{array}$ & $\begin{array}{l}\text { extension, } \\
\text { hotspot }\end{array}$ & Great Basin & $\begin{array}{l}\text { Glen and Ponce, 2002; } \\
\text { Ponce and Glen, 2002 }\end{array}$ \\
\hline
\end{tabular}


Table 1: Geophysical Features of the Great Basin and parts of the surrounding provinces

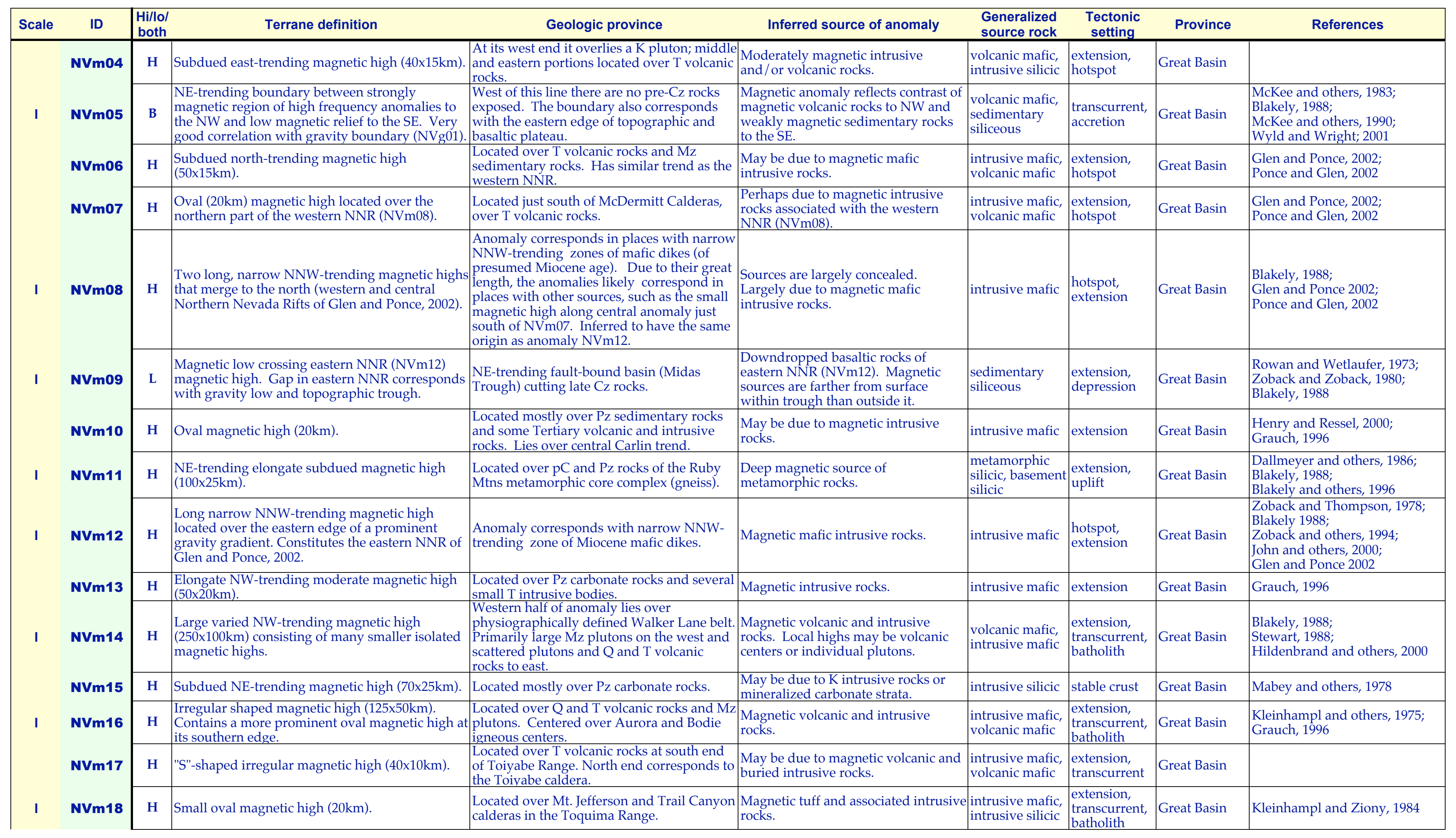


Table 1: Geophysical Features of the Great Basin and parts of the surrounding provinces

\begin{tabular}{|c|c|c|c|c|c|c|c|c|c|}
\hline Scale & ID & $\begin{array}{l}\text { Hi/lol } \\
\text { both }\end{array}$ & Terrane definition & Geologic province & Inferred source of anomaly & $\begin{array}{l}\text { Generalized } \\
\text { source rock }\end{array}$ & $\begin{array}{c}\text { Tectonic } \\
\text { setting } \\
\end{array}$ & Province & References \\
\hline \multirow[t]{2}{*}{ I } & NVm19 & $\mathbf{H}$ & $\begin{array}{l}\text { Mostly east-trending, irregular shaped region of } \\
\text { variable magnetic high }(125 \times 50 \mathrm{~km}) \text {. Contains a } \\
\text { more prominent oval magnetic high at its } \\
\text { southern edge. }\end{array}$ & $\begin{array}{l}\text { Located mostly over T volcanic rocks of } \\
\text { several identified volcanic centers. Few } \\
\text { scattered Mz plutons and Pz sedimentary } \\
\text { rocks. }\end{array}$ & $\begin{array}{l}\text { Magnetic volcanic and possibly } \\
\text { intrusive rocks. }\end{array}$ & $\begin{array}{l}\text { volcanic mafic, } \\
\text { volcanic silicic, } \\
\text { intrusive silicic }\end{array}$ & $\begin{array}{l}\text { extension, } \\
\text { subduction }\end{array}$ & Great Basin & \\
\hline & NVm20 & $\mathrm{H}$ & Oval moderate magnetic high $(30 \mathrm{~km})$ & $\begin{array}{l}\text { Located at east end of anomaly UTm15 and } \\
\text { over T volcanic rocks. }\end{array}$ & Magnetic volcanic rocks. & $\begin{array}{l}\text { volcanic mafic, } \\
\text { volcanic silicic }\end{array}$ & $\begin{array}{l}\text { extension, } \\
\text { subduction }\end{array}$ & Great Basin & Mabey and others, 1978 \\
\hline 1 & NVm21 & $\mathbf{H}$ & $\begin{array}{l}\text { Magnetic high }(25 \times 15 \mathrm{~km}) \text { located within broader } \\
\text { gravity low (NVg17). }\end{array}$ & $\begin{array}{l}\text { Wilson Creek Mtns - late Oligocene Indian } \\
\text { Peak and White Rock calderas. }\end{array}$ & $\begin{array}{l}\text { Thick accumulation of low density, } \\
\text { magnetic caldera fill tuffs and } \\
\text { associated intrusive rocks. }\end{array}$ & volcanic silicic & subduction & Great Basin & $\begin{array}{l}\text { Best and others, 1989a; } \\
\text { Best and others, 1989b }\end{array}$ \\
\hline 1 & NVm22 & $\mathbf{H}$ & $\begin{array}{l}\text { Subdued oval magnetic high mostly identified by } \\
\text { a ring of maximum horizontal gradients }(20 \mathrm{~km}) \text {. }\end{array}$ & $\begin{array}{l}\text { Located over } \mathrm{pC} \text { and Cambrian sedimentary } \\
\text { rocks and small exposure of } \mathrm{T} \text { volcanic } \\
\text { rocks. }\end{array}$ & $\begin{array}{l}\text { Oval form suggests it may be a buried } \\
\text { pluton. }\end{array}$ & intrusive silicic & $\begin{array}{l}\text { batholith, } \\
\text { transurrent }\end{array}$ & Great Basin & \\
\hline 1 & NVm23 & $\mathbf{H}$ & $\begin{array}{l}\text { NW-trending elongate region with high } \\
\text { frequency NW elongate positive anomalies. } \\
\text { Region is } \sim 250 \times 550 \mathrm{~km} \text { in western and central } \\
\text { NV. Includes several large anomalies (e.g. } \\
\text { NVm14,16,17,19,22,25). Southwest part of } \\
\text { anomaly located over gravity anomaly NVg11. }\end{array}$ & $\begin{array}{l}\text { Physiographically defined province } \\
\text { characterized by NW-trending topographic } \\
\text { features. located over Mz granites, Pz strata, } \\
\text { and late T volcanic rocks. Western part of } \\
\text { anomaly corresponds with the tectonically } \\
\text { defined Walker Lane. }\end{array}$ & $\begin{array}{l}\text { Gravity terrane is possibly due to } \\
\text { mafic underplating. Magnetic } \\
\text { anomalies may express shallow to } \\
\text { deeply buried magnetic plutons. } \\
\text { Possible represents the eastern edge } \\
\text { of Sierra Nevada Batholith. }\end{array}$ & intrusive mafic & $\begin{array}{l}\text { transcurrent, } \\
\text { extension, } \\
\text { continental } \\
\text { margin }\end{array}$ & Great Basin & $\begin{array}{l}\text { Blakely, 1988; } \\
\text { Craig, 1992; } \\
\text { Hildenbrand and others, 2000; } \\
\text { Blakely and others, } 1996\end{array}$ \\
\hline I & NVm24 & $\mathbf{H}$ & $\begin{array}{l}\text { A region of variable magnetic highs. EW-oriented } \\
\text { oval feature ( } 75 \mathrm{~km} \times 50 \mathrm{~km} \text { ) slightly displaced to } \\
\text { north of a similarly shaped gravity low (NVg20). }\end{array}$ & $\begin{array}{l}\text { Clover Mtns region - Miocene Caliente } \\
\text { caldera. }\end{array}$ & $\begin{array}{l}\text { Magnetic volcanic tuffs and } \\
\text { associated intrusive rocks. }\end{array}$ & $\begin{array}{l}\text { volcanic silicic, } \\
\text { intrusive silicic }\end{array}$ & subduction & Great Basin & $\begin{array}{l}\text { Best and others, 1989a; } \\
\text { Best and others, 1989b }\end{array}$ \\
\hline $\mathrm{I}$ & NVm25 & $\mathbf{H}$ & $\begin{array}{l}\text { NW-trending magnetic high }(90 \times 25 \mathrm{~km}) \text { located } \\
\text { over a basement gravity low. Corresponds partly } \\
\text { with the single largest isostatic gravity low in } \\
\text { Nevada (Silent Canyon Caldera). }\end{array}$ & $\begin{array}{l}\text { Located over the southern Nevada volcanic } \\
\text { field - Miocene silicic volcanic rocks. } \\
\text { Several identified calderas (e.g. Silent } \\
\text { Canyon, Timber Mtn, Black Mtn). }\end{array}$ & Magnetic, low density volcanic rocks. & volcanic silicic & $\begin{array}{l}\text { subduction, } \\
\text { transcurrent }\end{array}$ & Great Basin & $\begin{array}{l}\text { Eckel, 1968; } \\
\text { Byers and others, 1976; } \\
\text { Oliver and others, 1995; } \\
\text { Grauch and others, 1999; } \\
\text { Mankinen and others, 1999; } \\
\text { McKee and others, 1999; } \\
\text { Hildenbrand and others, 2000; } \\
\text { McKee and others, 2001 }\end{array}$ \\
\hline 1 & NVm26 & $\mathbf{H}$ & $\begin{array}{l}\text { Small pronounced oval magnetic high }(20 \mathrm{~km}) \text {. } \\
\text { Located over a much broader pronounced } \\
\text { gravity high (NVg23). }\end{array}$ & $\begin{array}{l}\text { Mormon Mtns--Fault block of pC crystalline } \\
\text { basement. }\end{array}$ & $\begin{array}{l}\text { Dense and magnetic crystalline } \\
\text { basement. }\end{array}$ & basement mafic & uplift & Great Basin & $\begin{array}{l}\text { Blank, 1988; } \\
\text { Blakely, 1988; } \\
\text { Blakely and others, } 1996\end{array}$ \\
\hline 1 & NVm27 & $\mathbf{H}$ & $\begin{array}{l}\text { Small subdued oval magnetic high }(40 \mathrm{~km}) \text {. } \\
\text { Located over a regional gravity high. }\end{array}$ & Fault block of pC crystalline basement. & $\begin{array}{l}\text { Dense and magnetic crystalline } \\
\text { basement. }\end{array}$ & basement mafic & uplift & Great Basin & $\begin{array}{l}\text { Blank, 1988; } \\
\text { Blakely, 1988; } \\
\text { Blakely and others, } 1996\end{array}$ \\
\hline 1 & NVm28 & $\mathrm{H}$ & $\begin{array}{l}\text { Small pronounced oval magnetic high }(20 \mathrm{~km}) \text {. } \\
\text { Located over a much broader pronounced } \\
\text { gravity high (NVg23). }\end{array}$ & Fault block of pC crystalline basement. & $\begin{array}{l}\text { Dense and magnetic crystalline } \\
\text { basement. }\end{array}$ & basement mafic & uplift & Great Basin & \\
\hline 1 & NVm29 & $\mathbf{H}$ & $\begin{array}{l}\text { EW alignment of magnetic highs that separates a } \\
\text { regional magnetic low to north from a region } \\
\text { dominated by several local magnetic highs to } \\
\text { south. }\end{array}$ & $\begin{array}{l}\text { South of this boundary are } Q \text { volcanic, } \mathrm{T} \\
\text { intrusive, and Mz granititic rocks. To the } \\
\text { north are mainly Pz carbonate rocks. }\end{array}$ & $\begin{array}{l}\text { Weakly magnetic carbonate rocks to } \\
\text { north and magnetic igneous rocks to } \\
\text { south. }\end{array}$ & $\begin{array}{l}\text { sedimentary } \\
\text { carbonate, } \\
\text { volcanic mafic, } \\
\text { basement mafic }\end{array}$ & $\begin{array}{l}\text { extension, } \\
\text { uplift }\end{array}$ & Great Basin & Blakely and others, 1996 \\
\hline $\mathbf{L}$ & NVm30 & $\mathbf{H}$ & $\begin{array}{l}\text { Small oval magnetic high (30km) enclosing a } \\
\text { smaller NS elongate high. Located over NW } \\
\text { flank of a broad NW-trending magnetic } \\
\text { high }(60 \times 30 \mathrm{~km}) \text {. Corresponds partly with gravity } \\
\text { high NVg22. }\end{array}$ & Fault block of $\mathrm{pC}$ to Mz sedimentary rocks. & $\begin{array}{l}\text { Possibly due to upwarp in dense and } \\
\text { magnetic crystalline basement and to } \\
\text { an associated T intrusion. }\end{array}$ & basement mafic & uplift & Great Basin & $\begin{array}{l}\text { Stewart and Carlson, 1978; } \\
\text { Blank, 1988; } \\
\text { Blakely, 1988; } \\
\text { Blakely and others, } 1996\end{array}$ \\
\hline 1 & ORg01 & $\mathbf{H}$ & $\begin{array}{l}\text { Subdued NS-elongate gravity high ( }>125 \mathrm{x} \\
>75 \mathrm{~km}) \text {. Corresponds with magnetic anomaly } \\
\text { ORm01. }\end{array}$ & $\begin{array}{l}\text { Located over western Cascades, late } \mathrm{Cz} \\
\text { volcanic rocks. Eastern edge is at the } \\
\text { transition between the basaltic western } \\
\text { Cascades and the younger, more andesitic } \\
\text { eastern Cascades. }\end{array}$ & $\begin{array}{l}\text { Interpreted to be due to concealed } \\
\text { accreted Eocene Siletz River volcanic } \\
\text { rocks. Forms part of the eastern edge } \\
\text { of the Oregon forearc block. }\end{array}$ & volcanic mafic & $\begin{array}{l}\text { accretion, } \\
\text { subduction }\end{array}$ & Cascade Mtns & Wells and others, 1998 \\
\hline
\end{tabular}


Table 1: Geophysical Features of the Great Basin and parts of the surrounding provinces

\begin{tabular}{|c|c|c|c|c|c|c|c|c|c|}
\hline Scale & ID & $\begin{array}{l}\mathrm{Hi} / \mathrm{lo} / \\
\text { both }\end{array}$ & Terrane definition & Geologic province & Inferred source of anomaly & $\begin{array}{l}\text { Generalized } \\
\text { source rock }\end{array}$ & $\begin{array}{l}\text { Tectonic } \\
\text { setting }\end{array}$ & Province & References \\
\hline I & ORg02 & $\mathbf{H}$ & $\begin{array}{l}\text { Prominent oval gravity high }(50 \mathrm{~km}) \text { located at } \\
\text { SW end of feature ORg03. Corresponds partly } \\
\text { with magnetic anomaly ORm07. }\end{array}$ & Located over $\mathrm{T}$ volcanic rocks. & $\begin{array}{l}\text { Perhaps due to dense magnetic } \\
\text { intrusive rocks. }\end{array}$ & $\begin{array}{l}\text { intrusive mafic, } \\
\text { volcanic mafic }\end{array}$ & hotspot & $\begin{array}{l}\text { Columbia } \\
\text { Plateaus }\end{array}$ & \\
\hline 1 & ORg03 & $\mathbf{H}$ & $\begin{array}{l}\text { NE-trending elongate gravity high ( }>125 \times 40 \mathrm{~km}) \\
\text { Corresponds with magnetic anomaly ORm03. }\end{array}$ & $\begin{array}{l}\text { Skirts north edge of Blue Mtns and located } \\
\text { mostly within the southern part of the John } \\
\text { Day basin. }\end{array}$ & $\begin{array}{l}\text { Perhaps due to dense magnetic rift } \\
\text { intrusive rocks or accreted Triassic } \\
\text { ultramafic rocks. }\end{array}$ & $\begin{array}{l}\text { intrusive mafic, } \\
\text { volcanic mafic, } \\
\text { ultramafic }\end{array}$ & $\begin{array}{l}\text { hotspot, } \\
\text { accretion }\end{array}$ & $\begin{array}{l}\text { Columbia } \\
\text { Plateaus }\end{array}$ & \\
\hline \multirow[t]{2}{*}{$\mathbf{R}$} & ORg04 & $\mathbf{L}$ & $\begin{array}{l}\text { NNW-elongate gravity low (50x175km). Closely } \\
\text { correlated with magnetic high ORm08. }\end{array}$ & $\begin{array}{l}\text { Western Cascade Range consisting of T } \\
\text { andesite volcanic rocks in the Cascade } \\
\text { graben. }\end{array}$ & $\begin{array}{l}\text { Magnetic, low density intermediate } \\
\text { volcanic rocks. }\end{array}$ & $\begin{array}{l}\text { volcanic silicic, } \\
\text { intrusive silicic }\end{array}$ & subduction & Cascade Mtns & $\begin{array}{l}\text { Jachens and others, 1989; } \\
\text { LaFehr, 1965; } \\
\text { Blakely and others, } 1985\end{array}$ \\
\hline & ORg05 & L & $\begin{array}{l}\text { NNW-trending gravity low }(100 \times 25 \mathrm{~km}) \\
\text { Contains discrete lows within it. }\end{array}$ & $\begin{array}{l}\text { Located over } \mathrm{Q} \text { and } \mathrm{T} \text { volcanic rocks of the } \\
\text { high Cascade Range. Includes Crater Lake. }\end{array}$ & $\begin{array}{l}\text { Magnetic, low density intermediate } \\
\text { volcanic rocks. }\end{array}$ & $\begin{array}{l}\text { volcanic silicic } \\
\text { intrusive silicic }\end{array}$ & subduction & $\begin{array}{l}\text { Cascade Mtns, } \\
\text { Columbia } \\
\text { Plateaus }\end{array}$ & \\
\hline $\mathbf{R}$ & ORg06 & B & $\begin{array}{l}\text { Moderate gravity low with a few small }(<100 \mathrm{~km} \\
\text { diameter) intermediate amplitude highs. } \\
\text { Regional low is mainly defined by highs to east } \\
\text { associated with northern GB and western SRP. } \\
\text { Corresponds in part with magnetic anomaly }\end{array}$ & $\begin{array}{l}\text { Southernmost edge of CRP basalts and } \\
\text { related lava flows, and younger lava flows } \\
\text { across Oregon highlands. lava flows are } \\
\text { underlain by Tertiary sediments. }\end{array}$ & Low density sedimentary rocks. & $\begin{array}{l}\text { sedimentary } \\
\text { siliceous }\end{array}$ & extension & $\begin{array}{l}\text { Columbia } \\
\text { Plateaus }\end{array}$ & \\
\hline \multirow[t]{2}{*}{ I } & ORm01 & $\mathrm{H}$ & $\begin{array}{l}\text { Pronounced magnetic high }(>75 \mathrm{~km} \text { NS } x>60 \mathrm{~km} \\
\text { EW). Occupies northern } 2 / 3 \text { of the } \\
\text { corresponding gravity anomaly. Corresponds } \\
\text { with gravity anomaly ORg01. }\end{array}$ & $\begin{array}{l}\text { Located over western Cascades, late } \mathrm{Cz} \\
\text { volcanic rocks. Eastern edge is at the } \\
\text { transition between the basaltic western } \\
\text { Cascades and the younger, more andesitic } \\
\text { eastern Cascades. }\end{array}$ & $\begin{array}{l}\text { Interpreted to be due to concealed } \\
\text { accreted Eocene Siletz River Volcanic } \\
\text { rocks. Forms part of the eastern edge } \\
\text { of the Oregon forearc block. }\end{array}$ & volcanic mafic & $\begin{array}{l}\text { accretion, } \\
\text { subduction }\end{array}$ & Cascade Mtns & Wells and others, 1998 \\
\hline & ORm02 & $\mathbf{H}$ & Elongate NS-oriented magnetic high $(50 \times 20 \mathrm{~km})$. & $\begin{array}{l}\text { Located over T and Q volcanic rocks of the } \\
\text { Cascade Range that includes the Three } \\
\text { Sisters and Mt. Washington. }\end{array}$ & Strongly magnetic volcanic rocks. & $\begin{array}{l}\text { volcanic silicic } \\
\text { intrusive silicic }\end{array}$ & subduction & $\begin{array}{l}\text { Cascade Mtns, } \\
\text { Columbia } \\
\text { Plateaus }\end{array}$ & \\
\hline \multirow{2}{*}{ I } & ORm04 & $\mathrm{L}$ & Large NE-trending magnetic low (225x60km). & $\begin{array}{l}\text { Corresponds closely with Mz sedimentary } \\
\text { rocks of the Blue Mtns. }\end{array}$ & \begin{tabular}{|l|} 
Weakly magnetic sedimentary rocks. \\
Mainly contrasted with the \\
surrounding strongly magnetic \\
volcanic rocks.
\end{tabular} & $\begin{array}{l}\text { sedimentary } \\
\text { siliceous }\end{array}$ & accretion & $\begin{array}{l}\text { Columbia } \\
\text { Plateaus, Great } \\
\text { Basin }\end{array}$ & $\begin{array}{l}\text { Vallier and Brooks, 1986; } \\
\text { Vallier and Brooks, } 1994\end{array}$ \\
\hline & ORm05 & $\mathbf{H}$ & $\begin{array}{l}\text { Elongate arcuate NS-trending magnetic high } \\
(100 \times 25 \mathrm{~km}) \text {. }\end{array}$ & $\begin{array}{l}\text { Located over } \mathrm{Q} \text { and } \mathrm{T} \text { volcanic rocks on the } \\
\text { east side of the Cascade Range (includes } \\
\text { Newberry Craters). }\end{array}$ & Strongly magnetic volcanic rocks. & $\begin{array}{l}\text { volcanic mafic, } \\
\text { intrusive mafic }\end{array}$ & subduction & $\begin{array}{l}\text { Columbia } \\
\text { Plateaus }\end{array}$ & \\
\hline $\mathbf{R}$ & ORm06 & B & \begin{tabular}{|l|} 
High amplitude, high frequency magnetic \\
anomalies that extend across Oregon highlands. \\
Corresponds with gravity anomaly ORg06.
\end{tabular} & $\begin{array}{l}\text { Southernmost edge of CRP basalts and } \\
\text { related rocks, and younger volcanic rocks } \\
\text { across Oregon highlands. Volcanic rocks are } \\
\text { underlain by Tertiary sediments. }\end{array}$ & Magnetic basaltic rocks. & volcanic mafic & $\begin{array}{l}\text { extension, } \\
\text { hotspot }\end{array}$ & $\begin{array}{l}\text { Columbia } \\
\text { Plateaus }\end{array}$ & \\
\hline 1 & ORm07 & H & $\begin{array}{l}\text { Oval magnetic high }(40 \mathrm{~km}) \text { that is located at the } \\
\text { SW end of feature ORm03. Corresponds partly } \\
\text { with gravity anomaly } \mathrm{ORg} 02 \text {. }\end{array}$ & Located over $\mathrm{T}$ volcanic rocks. & $\begin{array}{l}\text { Perhaps due to dense magnetic } \\
\text { intrusive rocks. }\end{array}$ & $\begin{array}{l}\text { intrusive mafic, } \\
\text { volcanic mafic }\end{array}$ & hotspot & $\begin{array}{l}\text { Columbia } \\
\text { Plateaus }\end{array}$ & \\
\hline \multirow[t]{4}{*}{$\mathbf{R}$} & ORm08 & $\mathbf{H}$ & $\begin{array}{l}\text { NNW-elongate magnetic high }(50 \times 200 \mathrm{~km}) \text {. } \\
\text { Closely correlated with gravity high ORg04. } \\
\text { Consists of high amplitude and high frequency } \\
\text { magnetic anomalies. }\end{array}$ & $\begin{array}{l}\text { Western Cascade Range consisting of } \mathrm{T} \\
\text { andesite in the Cascade graben. }\end{array}$ & $\begin{array}{l}\text { Magnetic, low density intermediate } \\
\text { volcanic rocks. }\end{array}$ & $\begin{array}{l}\text { volcanic silicic, } \\
\text { intrusive silicic }\end{array}$ & subduction & Cascade Mtns & $\begin{array}{l}\text { LaFehr, 1965; } \\
\text { Blakely and others, 1985; } \\
\text { Jachens and others, } 1989\end{array}$ \\
\hline & ORm09 & $\mathrm{H}$ & Elongate NW-trending magnetic high (60x10km). & $\begin{array}{l}\text { Located over } \mathrm{Q} \text { and } \mathrm{T} \text { volcanic and } \\
\text { sedimentary rocks. Anomaly has the same } \\
\text { trend as Q faulting. }\end{array}$ & Strongly magnetic volcanic rocks. & $\begin{array}{l}\text { volcanic mafic, } \\
\text { intrusive mafic }\end{array}$ & $\begin{array}{l}\text { extension, } \\
\text { hotspot }\end{array}$ & $\begin{array}{l}\text { Columbia } \\
\text { Plateaus }\end{array}$ & \\
\hline & ORm10 & $\mathrm{H}$ & Elongate NW-trending magnetic high $(50 \times 20 \mathrm{~km})$. & $\begin{array}{l}\text { Located over T volcanic and sedimentary } \\
\text { rocks. Anomaly has the same trend as Q } \\
\text { faulting. }\end{array}$ & Strongly magnetic volcanic rocks. & $\begin{array}{l}\text { volcanic mafic, } \\
\text { intrusive mafic }\end{array}$ & $\begin{array}{l}\text { extension, } \\
\text { hotspot }\end{array}$ & $\begin{array}{l}\text { Columbia } \\
\text { Plateaus }\end{array}$ & \\
\hline & ORm11 & $\mathbf{H}$ & $\begin{array}{l}\text { Elongate north-trending magnetic high } \\
(80 \times 20 \mathrm{~km}) \text {. }\end{array}$ & Located over $\mathrm{Q}$ and $\mathrm{T}$ volcanic rocks. & Strongly magnetic volcanic rocks. & $\begin{array}{l}\text { volcanic mafic, } \\
\text { intrusive mafic }\end{array}$ & $\begin{array}{l}\text { extension, } \\
\text { hotspot }\end{array}$ & Great Basin & \\
\hline
\end{tabular}




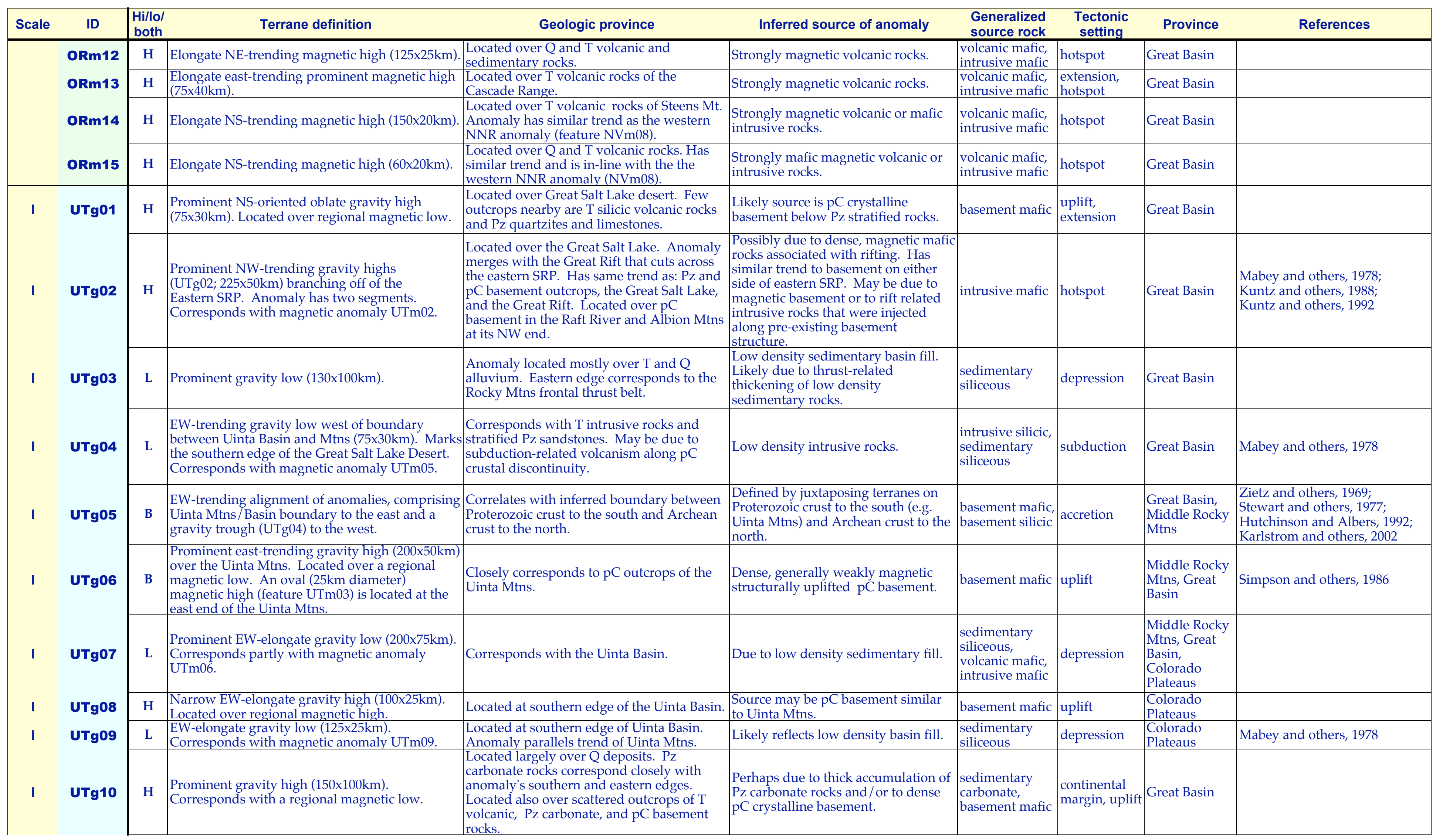




\begin{tabular}{|c|c|c|c|c|c|c|c|c|c|}
\hline Scale & ID & $\begin{array}{l}\mathrm{Hi} / \mathrm{lo} / \\
\text { both }\end{array}$ & Terrane definition & Geologic province & Inferred source of anomaly & $\begin{array}{l}\text { Generalized } \\
\text { source rock }\end{array}$ & $\begin{array}{l}\text { Tectonic } \\
\text { setting }\end{array}$ & Province & References \\
\hline I & UTg11 & $\mathrm{H}$ & $\begin{array}{l}\text { NE-trending gravity high }(150 \times 50 \mathrm{~km}) \text { that } \\
\text { encompasses the Wasatch Plateau. Northern end } \\
\text { located over a magnetic low and southern end } \\
\text { over a magnetic high (UTm11). }\end{array}$ & \begin{tabular}{|l|} 
Located at north end of basin along the \\
Sevier River over western Colorado Plateau. \\
Corresponds with the western part of the \\
San Rafel Swell.
\end{tabular} & $\begin{array}{l}\text { Likely reflects upwarp of dense pC } \\
\text { basement. }\end{array}$ & basement mafic & uplift & $\begin{array}{l}\text { Colorado } \\
\text { Plateaus, Great } \\
\text { Basin }\end{array}$ & \\
\hline 1 & UTg12 & $\mathbf{L}$ & $\begin{array}{l}\text { NW-trending gravity low }(125 \times 50 \mathrm{~km}) \text { with } \\
\text { distinct NW-trending ridges. Located over a } \\
\text { generally low magnetic area. }\end{array}$ & $\begin{array}{l}\text { Located over La Sal Mtns. Straddles } \\
\text { Northern edge of CRP. }\end{array}$ & $\begin{array}{l}\text { Low may be partly due to La Sal T } \\
\text { intrusive bodies. Ridges may be } \\
\text { related to faults in pC basement. }\end{array}$ & intrusive silicic & stable crust & $\begin{array}{l}\text { Colorado } \\
\text { Plateaus }\end{array}$ & \\
\hline 1 & UTg13 & $\mathrm{H}$ & $\begin{array}{l}\text { NE-trending gravity high }(170 \times 50 \mathrm{~km}) \text {. } \\
\text { Corresponds with magnetic anomaly UTm14. }\end{array}$ & $\begin{array}{l}\text { Roughly corresponds to the edge of the } \\
\text { Wasatch plateau. Cuts across drainages of } \\
\text { the CP. Located over part of the Circle Cliffs } \\
\text { and San Rafael Swell structural uplifts. }\end{array}$ & $\begin{array}{l}\text { Likely reflects upwarp of dense, } \\
\text { magnetic } \mathrm{PC} \text { basement. }\end{array}$ & basement mafic & uplift & $\begin{array}{l}\text { Colorado } \\
\text { Plateaus }\end{array}$ & \\
\hline 1 & UTg14 & $\mathrm{H}$ & $\begin{array}{l}\text { NS-oriented gravity high }(100 \times 40 \mathrm{~km}) \text { that is } \\
\text { contiguous with feature UTg10. North end } \\
\text { crosses large magnetic high (UTm15). South end } \\
\text { located over magnetic low region. }\end{array}$ & $\begin{array}{l}\text { Located at edge of GB over the eastern edge } \\
\text { of the Sevier Thrust belt. Located mostly } \\
\text { over T volcanic rocks. }\end{array}$ & $\begin{array}{l}\text { Unknown source, but possibly dense } \\
\text { basement brought near to surface by } \\
\text { frontal thrust. }\end{array}$ & basement mafic & uplift & Great Basin & \\
\hline 1 & UTg15 & L & $\begin{array}{l}\text { Oval gravity low }(50 \mathrm{~km}) . \text { Located over a } \\
\text { magnetic low. }\end{array}$ & $\begin{array}{l}\text { Located over welded silicic tuff volcanic } \\
\text { field (Bull Valley silicic complex). }\end{array}$ & $\begin{array}{l}\text { Thick accumulation of low density } \\
\text { rhyolitic and tufaceous sedimentary } \\
\text { rocks. }\end{array}$ & $\begin{array}{l}\text { volcanic silicic, } \\
\text { intrusive silicic }\end{array}$ & subduction & Great Basin & \\
\hline 1 & UTg16 & $\mathbf{L}$ & $\begin{array}{l}\text { Large oval gravity low }(100 \mathrm{~km}) \text {. Located over } \\
\text { moderately high magnetic terrain that includes } \\
\text { magnetic anomalies AZm02 and southern end of } \\
\text { UTm14. }\end{array}$ & $\begin{array}{l}\text { Located over Mz and Pz strata of CRP over } \\
\text { Glen Canyon along the Colorado River. }\end{array}$ & $\begin{array}{l}\text { Area is covered by CRP strata. } \\
\text { Possibly due to downwarp of } \\
\text { basement. }\end{array}$ & basement silicic & depression & $\begin{array}{l}\text { Colorado } \\
\text { Plateaus }\end{array}$ & \\
\hline 1 & UTg17 & $\mathrm{H}$ & $\begin{array}{l}\text { Gravity high }(100 \times 125 \mathrm{~km}) \text {. Encompasses feature } \\
\text { UTg18. Is contiguous with broader gravity high } \\
\text { (AZg04). Corresponds with magnetic anomaly } \\
\text { UTm21. }\end{array}$ & $\begin{array}{l}\text { Located over flat lying Pz and Mz strata of } \\
\text { CRP. Outlines the Monument upwarp. }\end{array}$ & $\begin{array}{l}\text { May be dense and magnetic intrusive } \\
\text { or uplifted buried basement rocks. }\end{array}$ & $\begin{array}{l}\text { intrusive mafic, } \\
\text { basement mafic }\end{array}$ & uplift & $\begin{array}{l}\text { Colorado } \\
\text { Plateaus }\end{array}$ & \\
\hline 1 & UTg18 & B & $\begin{array}{l}\text { Doughnut-shaped gravity high with internal low } \\
(50 \mathrm{~km}) \text {. Very good correlation with magnetic } \\
\text { anomaly UTm22. }\end{array}$ & $\begin{array}{l}\text { Located over Pz and Mz flat lying strata of } \\
\text { CRP, but corresponds with a small T } \\
\text { intrusive outcrop (much smaller) that } \\
\text { represents a deep seated intrusive or } \\
\text { diatreme. }\end{array}$ & $\begin{array}{l}\text { May be dense magnetic mantle } \\
\text { derived intrusive rocks. }\end{array}$ & $\begin{array}{l}\text { intrusive mafic, } \\
\text { ultramafic }\end{array}$ & stable crust & $\begin{array}{l}\text { Colorado } \\
\text { Plateaus }\end{array}$ & Mabey and others, 1978 \\
\hline $\mathbf{R}$ & UTm01 & L & $\begin{array}{l}\text { Regional magnetic low lacking short-wavelength } \\
\text { magnetic anomalies. Extends from the Walker } \\
\text { Lane (NVm23) and Modoc Plateau (CAm02) } \\
\text { anomalies on the west eastward across virtually } \\
\text { the entire study area. Terrane extends from the } \\
\text { SRP (IDm10) to the southern Quiet zone } \\
\text { boundary (NVm29) and edge of the Colorado } \\
\text { Plateaus (UTm12). }\end{array}$ & $\begin{array}{l}\text { Anomaly spans a wide range of rock types } \\
\text { and ages. }\end{array}$ & $\begin{array}{l}\text { Speculation on source includes initial } \\
\text { low magnetic susceptibilities of } \\
\text { volcanic rocks (Stewart and others, } \\
\text { 1977; Blakely, 1988), diminished } \\
\text { magnetic susceptibility by intense } \\
\text { hydrothermal alteration (Eaton, 1978). } \\
\text { Demonstrated (Blakely, 1988) not to } \\
\text { be the result of a shallow Curie } \\
\text { isotherm. }\end{array}$ & $\begin{array}{l}\text { volcanic silicic, } \\
\text { metamorphic }\end{array}$ & $\begin{array}{l}\text { subduction, } \\
\text { extension }\end{array}$ & Great Basin & $\begin{array}{l}\text { Stewart and others, 1977; } \\
\text { Eaton and others, 1978; } \\
\text { Mabey and others, 1978; } \\
\text { Blakely, 1988 }\end{array}$ \\
\hline 1 & UTm02 & $\mathrm{H}$ & $\begin{array}{l}\text { NW-trending magnetic high }(225 \times 50 \mathrm{~km}) \text {. Extends } \\
\text { SE from the eastern SRP. Corresponds with } \\
\text { gravity anomaly UTg02. }\end{array}$ & $\begin{array}{l}\text { Located over the Great Salt Lake. Anomaly } \\
\text { merges with the Great Rift that cuts across } \\
\text { the eastern SRP. Has same trend as: Pz and } \\
\text { pC basement outcrops, the Great Salt Lake, } \\
\text { and the Great Rift. Located over pC } \\
\text { bsement in the Raft River and Albion Mtns } \\
\text { at its NW end. }\end{array}$ & \begin{tabular}{|l|} 
Possibly due to dense, magnetic mafic \\
rocks associated with rifting. Has \\
similar trend to basement on either \\
side of eastern SRP. May be due to \\
magnetic basement or to rift related \\
intrusive rocks that were injected \\
along pre-existing basement \\
structure.
\end{tabular} & intrusive mafic & hotspot & Great Basin & $\begin{array}{l}\text { Mabey and others, 1978; } \\
\text { Kuntz and others, 1988; } \\
\text { Kuntz and others, 1992 }\end{array}$ \\
\hline 1 & UTm03 & B & $\begin{array}{l}\text { An oval }(25 \mathrm{~km} \text { diameter) magnetic high located } \\
\text { at the east end of the Uinta Mtns. }\end{array}$ & $\begin{array}{l}\text { Closely corresponds to pC outcrops of the } \\
\text { Uinta Mtns. }\end{array}$ & $\begin{array}{l}\text { Magnetic structurally uplifted } \mathrm{pC} \\
\text { basement. }\end{array}$ & basement mafic & uplift & $\begin{array}{l}\text { Middle Rocky } \\
\text { Mtns, Great } \\
\text { Basin }\end{array}$ & Simpson and others, 1986 \\
\hline
\end{tabular}


Table 1: Geophysical Features of the Great Basin and parts of the surrounding provinces

\begin{tabular}{|c|c|c|c|c|c|c|c|c|c|}
\hline Scale & ID & $\begin{array}{l}\mathrm{Hi} / \mathrm{lo} / \\
\text { both }\end{array}$ & Terrane definition & Geologic province & Inferred source of anomaly & $\begin{array}{l}\text { Generalized } \\
\text { source rock }\end{array}$ & $\begin{array}{l}\text { Tectonic } \\
\text { setting }\end{array}$ & Province & References \\
\hline I & UTm04 & $\mathbf{H}$ & $\begin{array}{l}\text { Moderate NW-trending magnetic high } \\
(75 \times 30 \mathrm{~km}) \text {. }\end{array}$ & $\begin{array}{l}\text { Located over Q fill of Great Salt Lake Desert. } \\
\text { Nearest outcrops are volcanic rocks and pC } \\
\text { basement. Mz Gold Hill pluton lies to the } \\
\text { south. }\end{array}$ & $\begin{array}{l}\text { Possibly due to magnetic pluton, } \\
\text { magnetic basement, or volcanic } \\
\text { rocks. }\end{array}$ & $\begin{array}{l}\text { intrusive mafic, } \\
\text { basement mafic, } \\
\text { volcanic mafic }\end{array}$ & $\begin{array}{l}\text { subduction, } \\
\text { extension }\end{array}$ & Great Basin & Mabey and others, 1978 \\
\hline I & UTm05 & H & $\begin{array}{l}\text { ENE-trending narrow linear magnetic high } \\
\text { (30x5km) west of boundary between Uinta Basin } \\
\text { and Mtns (feature UTm05). Marks the southern } \\
\text { edge of the Great Salt Lake Desert. Corresponds } \\
\text { with gravity anomaly UTg04. }\end{array}$ & $\begin{array}{l}\text { Corresponds with several T intrusive rocks } \\
\text { (Wasatch igneous belt) and stratified Pz } \\
\text { sandstones. May be due to subduction- } \\
\text { related volcanism along pC crustal } \\
\text { discontinuity. }\end{array}$ & Magnetic, low density intrusive rocks. & $\begin{array}{l}\text { intrusive silicic, } \\
\text { sedimentary } \\
\text { siliceous }\end{array}$ & subduction & Great Basin & $\begin{array}{l}\text { Mabey and others, 1978; } \\
\text { Vogel and others, } 2001\end{array}$ \\
\hline I & UTm06 & $\mathbf{H}$ & $\begin{array}{l}\text { WNW-elongate oval-shaped magnetic high } \\
\text { (75x40km) that lies within a gravity low. } \\
\text { Corresponds with gravity anomaly UTg07. }\end{array}$ & Corresponds with part of the Uinta Basin. & $\begin{array}{l}\text { Source is likely buried magnetic } \\
\text { volcanic or intrusive rocks. }\end{array}$ & $\begin{array}{l}\text { sedimentary } \\
\text { siliceous, } \\
\text { volcanic mafic, } \\
\text { intrusive mafic }\end{array}$ & depression & $\begin{array}{l}\text { Middle Rocky } \\
\text { Mtns, Great } \\
\text { Basin, } \\
\text { Colorado } \\
\text { Plateaus } \\
\end{array}$ & \\
\hline & UTm07 & $\mathbf{H}$ & Elongate moderate magnetic high $(50 \times 25 \mathrm{~km})$. & $\begin{array}{l}\text { Located over outcrops of } \mathrm{pC} \text { and } \mathrm{Pz} \\
\text { sedimentary, and T volcanic and intrusive } \\
\text { rocks. }\end{array}$ & $\begin{array}{l}\text { Magnetic Mz and } \mathrm{T} \text { intrusive rocks } \\
\text { and/or T volcanic rocks. }\end{array}$ & $\begin{array}{l}\text { intrusive mafic, } \\
\text { volcanic mafic }\end{array}$ & $\begin{array}{l}\text { subduction, } \\
\text { extension }\end{array}$ & Great Basin & Mabey and others, 1978 \\
\hline I & UTm08 & $\mathbf{H}$ & $\begin{array}{l}\text { EW-elongate magnetic high }(100 \times 40 \mathrm{~km}) \text {. Located } \\
\text { over a relative gravity low that is within a } \\
\text { regional gravity high. }\end{array}$ & $\begin{array}{l}\text { Located over } \mathrm{pC} \text { and Pz sedimentary and } \mathrm{T} \\
\text { volcanic rocks. }\end{array}$ & $\begin{array}{l}\text { May be due to magnetic basement } \\
\text { and/or volcanic and associated } \\
\text { intrusive rocks. }\end{array}$ & $\begin{array}{l}\text { basement mafic, } \\
\text { volcanic mafic, } \\
\text { intrusive mafic }\end{array}$ & $\begin{array}{l}\text { subduction, } \\
\text { extension }\end{array}$ & Great Basin & Mabey and others, 1978 \\
\hline I & UTm09 & $\mathrm{H}$ & $\begin{array}{l}\text { EW-elongate magnetic high }(125 \times 25 \mathrm{~km}) \text {. } \\
\text { Magnetic terrane overlaps with, but generally } \\
\text { located south of gravity anomaly UTg09. }\end{array}$ & $\begin{array}{l}\text { Located at southern edge of Uinta Basin. } \\
\text { Anomaly parallels trend of Uinta Mtns. }\end{array}$ & $\begin{array}{l}\text { Magnetic high may reflect magnetic } \\
\text { igneous rocks. }\end{array}$ & intrusive mafic & depression & $\begin{array}{l}\text { Colorado } \\
\text { Plateaus }\end{array}$ & Mabey and others, 1978 \\
\hline I & UTm10 & $\mathbf{H}$ & $\begin{array}{l}\text { Irregular magnetic high }(75 \times 75 \mathrm{~km}) \text { that includes } \\
\text { magnetic highs UTm06,09, and an unlabeled high } \\
\text { to the east. Anomaly spans several distinct EW- } \\
\text { trending gravity highs and lows (UTg07,08,09). }\end{array}$ & $\begin{array}{l}\text { Located mostly over the Uinta Basin over T } \\
\text { sedimentary rocks. }\end{array}$ & $\begin{array}{l}\text { Mostl likely due to magnetic } \\
\text { basement rocks. }\end{array}$ & basement mafic & stable crust & $\begin{array}{l}\text { Colorado } \\
\text { Plateaus }\end{array}$ & \\
\hline 1 & UTm11 & $\mathrm{H}$ & Oval magnetic high $(75 \times 50 \mathrm{~km})$ & $\begin{array}{l}\text { Located at north end of Sevier volcanic } \\
\text { plateau. }\end{array}$ & Magnetic volcanic rocks. & volcanic mafic & subduction & $\begin{array}{l}\text { Great Basin, } \\
\text { Colorado } \\
\text { Plateaus }\end{array}$ & \\
\hline 1 & UTm12 & B & $\begin{array}{l}\text { Boundary between regional low of GB and } \\
\text { regional high of } C P \text {. }\end{array}$ & Boundary, in most places, skirts the CP. & $\begin{array}{l}\text { Marks the boundary between } \\
\text { generally low magnetic terrain of the } \\
\text { GB and the varied, but strongly } \\
\text { magnetic terrain of the CP. CP } \\
\text { magnetic highs are largely due to } \\
\text { zones of magnetic basement. } \\
\end{array}$ & basement mafic & $\begin{array}{l}\text { stable crust, } \\
\text { extension }\end{array}$ & $\begin{array}{l}\text { Great Basin, } \\
\text { Colorado } \\
\text { Plateaus }\end{array}$ & \\
\hline & UTm13 & L & $\begin{array}{l}\text { Elongate east-trending magnetic low }(50 \times 25 \mathrm{~km}) \\
\text { with well defined northern and southern } \\
\text { boundaries. Located over a gravity low. }\end{array}$ & $\begin{array}{l}\text { Southern edge of the anomaly corresponds } \\
\text { with northern extent of a volcanic field } \\
\text { bordering the CP. Located over Q basin } \\
\text { with some outcrops of Pz and } \mathrm{Mz} \\
\text { sedimentary rocks. }\end{array}$ & \begin{tabular}{|l|} 
Northern edge may reflect basement \\
topography at bordering a Q basin. \\
Southern edge defined by contrast of \\
weakly magnetic basin fill with \\
magnetic T volcanic rocks.
\end{tabular} & $\begin{array}{l}\text { basement mafic, } \\
\text { sedimentary } \\
\text { siliceous, } \\
\text { volcanic mafic } \\
\end{array}$ & $\begin{array}{l}\text { subduction, } \\
\text { extension }\end{array}$ & Great Basin & \\
\hline 1 & UTm14 & $\mathbf{H}$ & $\begin{array}{l}\text { Arcuate NE-trending elongate magnetic high } \\
(300 \times 40 \mathrm{~km}) \text {. Corresponds with gravity anomaly } \\
\text { UTm13. }\end{array}$ & $\begin{array}{l}\text { Roughly corresponds to the edge of the } \\
\text { Wasatch plateau. Cuts across drainages of } \\
\text { the CP. Located over part of the Circle Cliffs } \\
\text { and San Rafel Swell structural uplifts. }\end{array}$ & $\begin{array}{l}\text { Likely reflects upwarp of dense, } \\
\text { magnetic PC basement. }\end{array}$ & basement mafic & uplift & $\begin{array}{l}\text { Colorado } \\
\text { Plateaus }\end{array}$ & \\
\hline 1 & UTm15 & $\mathbf{H}$ & $\begin{array}{l}\text { Elongate ENE-trending magnetic high } \\
(250 \times 30 \mathrm{~km}) \text {. }\end{array}$ & $\begin{array}{l}\text { Corresponds to Enterprise tholeitic basalts. } \\
\text { Eastern part of anomaly corresponds with a } \\
\text { T intrusive body. }\end{array}$ & $\begin{array}{l}\text { Strongly magnetic volcanic and } \\
\text { intrusive rocks. }\end{array}$ & $\begin{array}{l}\text { volcanic mafic, } \\
\text { intrusive mafic }\end{array}$ & $\begin{array}{l}\text { subduction, } \\
\text { extension }\end{array}$ & Great Basin & $\begin{array}{l}\text { Mabey and others, 1978; } \\
\text { Best and others, } 1980\end{array}$ \\
\hline
\end{tabular}


Table 1: Geophysical Features of the Great Basin and parts of the surrounding provinces

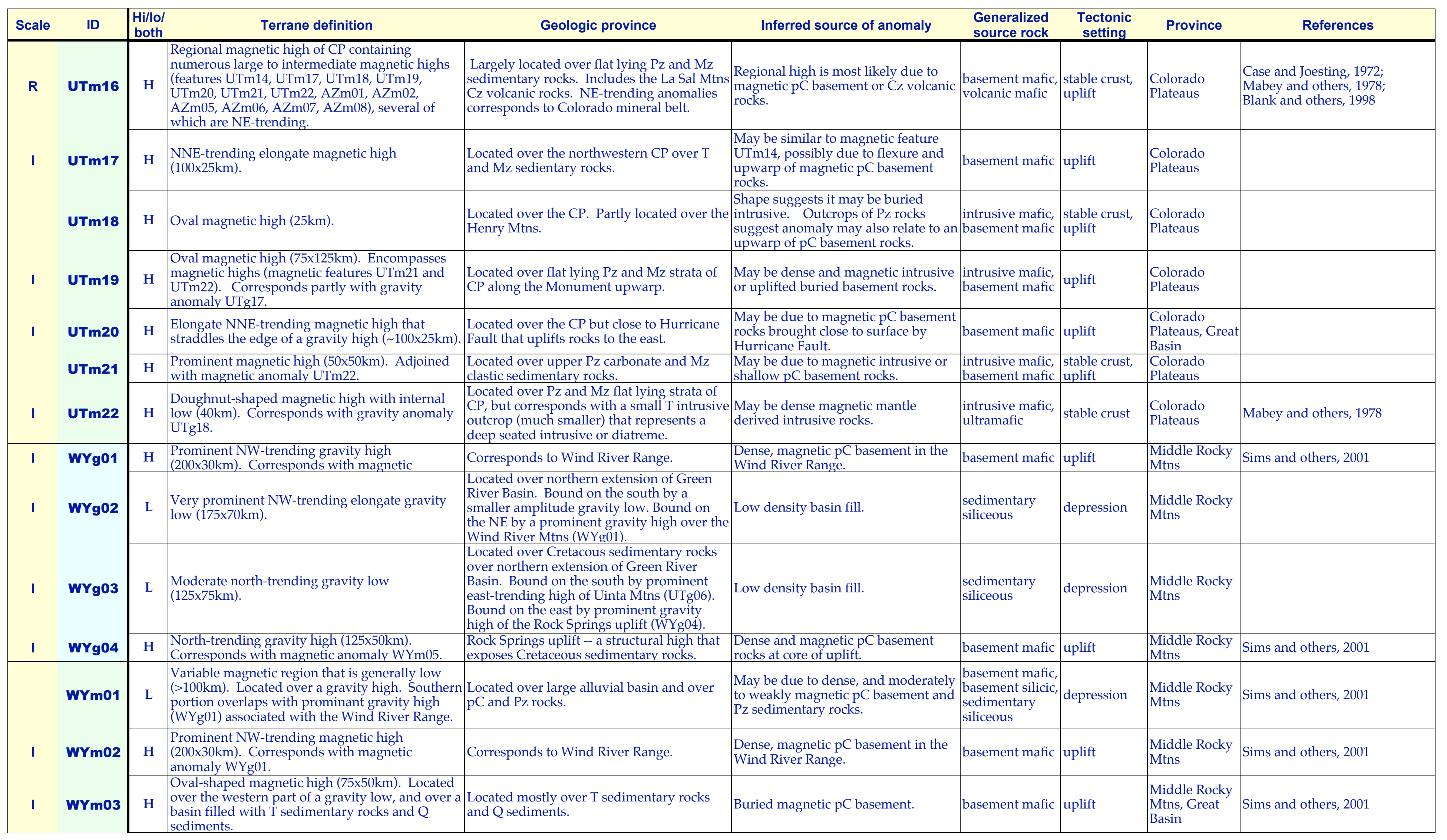


Table 1: Geophysical Features of the Great Basin and parts of the surrounding provinces

\begin{tabular}{|c|c|c|c|c|c|c|c|c|c|}
\hline Scale & ID & $\begin{array}{l}\mathrm{H} \text { Hi/lo/ } \\
\text { both }\end{array}$ & Terrane definition & Geologic province & Inferred source of anomaly & $\begin{array}{l}\text { Generalized } \\
\text { source rock }\end{array}$ & $\begin{array}{c}\text { Tectonic } \\
\text { setting }\end{array}$ & Province & References \\
\hline 1 & WYm04 & $\mathrm{L}$ & $\begin{array}{l}\text { Oval magnetic low }(60 \mathrm{~km}) \text {. Surrounded partly by } \\
\text { magnetic high feature WYm06. }\end{array}$ & $\begin{array}{l}\text { Located over Cretaceous sedimentary strata } \\
\text { in Green River basin. }\end{array}$ & $\begin{array}{l}\text { Weakly magnetic pC } \\
\text { metasedimentary rocks. }\end{array}$ & basement silicic & depression & $\begin{array}{l}\text { Great Basin, } \\
\text { Middle Rocky } \\
\text { Mtns }\end{array}$ & Sims and others, 2001 \\
\hline $\mathrm{I}$ & WYm05 & $\mathrm{H}$ & $\begin{array}{l}\text { North-trending low to moderate magnetic high } \\
(125 \times 25 \mathrm{~km}) \text {. Corresponds with magnetic } \\
\text { anomaly WYg04. }\end{array}$ & $\begin{array}{l}\text { Rock Springs uplift -- a structural high that } \\
\text { exposes Cretaceous sedimentary rocks. }\end{array}$ & $\begin{array}{l}\text { Dense and magnetic pC basement } \\
\text { rocks at core of uplift. }\end{array}$ & basement mafic & uplift & $\begin{array}{l}\text { Middle Rocky } \\
\text { Mtns }\end{array}$ & Sims and others, 2001 \\
\hline 1 & WYm06 & $\mathbf{H}$ & $\begin{array}{l}\text { EW-elongate, U-shaped magnetic high } \\
\text { (175x50km). Located north of Uinta Mtns, over } \\
\text { alluvium and T sedimentary rocks in the Flaming } \\
\text { Gorge area. }\end{array}$ & Located over Cretacous sedimentary strata. & Magnetic $\mathrm{pC}$ granitic rocks. & $\begin{array}{l}\text { intrusive silicic, } \\
\text { intrusive mafic, } \\
\text { basement mafic }\end{array}$ & $\begin{array}{l}\text { batholith, } \\
\text { uplift }\end{array}$ & $\begin{array}{l}\text { Middle Rocky } \\
\text { Mtns, Great } \\
\text { Basin }\end{array}$ & Sims and others, 2001 \\
\hline
\end{tabular}

(1) Generalized source rock: sedimentary - carbonate, siliceous; volcanic - silicic, mafic; intrusive - silicic, mafic; basement - silicic, mafic, carbonate; metamorphic -

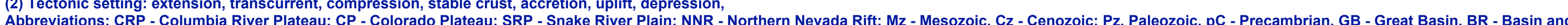
Range; compass directions (e.g. NE - northeast) 
Table 2. Physiograpic provinces and their geologic and geophysical character.

\begin{tabular}{|c|c|c|c|c|c|c|c|c|}
\hline $\begin{array}{l}\text { Physiographic } \\
\text { Province }\end{array}$ & Physiographic Description & Geologic Description & Gravity expression & Magnetic expression & $\begin{array}{c}\text { Generalized } \\
\text { geophysical } \\
\text { sources } \\
\end{array}$ & $\begin{array}{l}\text { General } \\
\text { tectonic } \\
\text { setting }\end{array}$ & Notes & References \\
\hline Cascade Mtns & $\begin{array}{l}\text { Part of the Cascade-Sierra Mountain } \\
\text { chain Province of Fenneman (1931). } \\
\text { Includes Southern and Middle Cascade } \\
\text { Mountains. In south, consists of } \\
\text { volcanic mountains, variously eroded, } \\
\text { and no distinct range. In the north, } \\
\text { consists of continuous range capped by } \\
\text { high volcanic plateau. }\end{array}$ & $\begin{array}{l}\text { Cenozoic to present subduction- } \\
\text { related volcanic arc. }\end{array}$ & $\begin{array}{l}\text { Characterized by low gravity } \\
\text { values. }\end{array}$ & $\begin{array}{l}\text { Dominated by high amplitude } \\
\text { and high frequency anomalies. }\end{array}$ & $\begin{array}{l}\text { Magnetic } \\
\text { volcanic rocks. }\end{array}$ & Subduction & $\begin{array}{l}\text { Contains no continental basement. Characterized } \\
\text { by high heat flow. Crustal thickness is on the } \\
\text { order of } 35-40 \mathrm{~km} \text {. }\end{array}$ & $\begin{array}{l}\text { Mabey and others, 1978; } \\
\text { Eaton and others 1978; } \\
\text { Smith, 1978; } \\
\text { Blackwell, } 1978\end{array}$ \\
\hline Coast Ranges & $\begin{array}{l}\text { Part of the Pacific Border Province of } \\
\text { Fenneman (1931). Parallel ranges and } \\
\text { valleys. Folded, faulted and } \\
\text { metamorphosed strata. Rounded crests } \\
\text { of unequal height. }\end{array}$ & $\begin{array}{l}\text { Northwest trending ranges } \\
\text { influenced by San Andreas fault } \\
\text { system. Consists of various fault } \\
\text { bounded tectonic slivers. Largely } \\
\text { sounsits of dissected Cenozoic } \\
\text { condimentary basins, Mesozoic } \\
\text { sedimites, melange. }\end{array}$ & $\begin{array}{l}\text { Characterized by numerous long } \\
\text { and narrow NNW-trending } \\
\text { gravity highs and lows. }\end{array}$ & $\begin{array}{l}\text { Characterized by numerous long } \\
\text { and narrow NNW-trending } \\
\text { magnetic highs and lows. }\end{array}$ & $\begin{array}{l}\text { Sedimentary } \\
\text { basins, ophiolite } \\
\text { rocks, intrusive } \\
\text { rocks. }\end{array}$ & $\begin{array}{l}\text { Accretion, } \\
\text { Transcurrent }\end{array}$ & $\begin{array}{l}\text { Characterized by variable high heat flow. } \\
\text { Especially high north of San Francisco associated } \\
\text { with Sonoma volcanic field. Crustal thickness is } \\
\text { on the order of 25-30km. Active seismically } \\
\text { associated with right-lateral transform motion } \\
\text { along the San Andreas Fault system. }\end{array}$ & $\begin{array}{l}\text { Smith, 1978; } \\
\text { Blackwell, } 1978\end{array}$ \\
\hline $\begin{array}{l}\text { Colorado } \\
\text { Plateaus }\end{array}$ & $\begin{array}{l}\text { Characteristics include: high block and } \\
\text { dissected plateaus, strong relief, and } \\
\text { mature canyon plateaus. In south, } \\
\text { trenched by Grand Canyon. }\end{array}$ & $\begin{array}{l}\text { Large stable crustal block overlain by } \\
\text { largely flat-lying Paleozoic and } \\
\text { Mesozoic strata with gentle flexures } \\
\text { forming broad north-south-trending } \\
\text { monoclinal folds. In placess, pierced } \\
\text { by deep-seated diatremes and } \\
\text { volcanic necks. Earthquakes are rare. }\end{array}$ & $\begin{array}{l}\text { Variable gravity signature } \\
\text { containing several gravity highs } \\
\text { and lows some reflecting a NE- } \\
\text { trending fabric. }\end{array}$ & $\begin{array}{l}\text { Characterized by several } \\
\text { prominent NE-trending magnetic } \\
\text { highs. }\end{array}$ & $\begin{array}{l}\text { Magnetic } \\
\text { c intrusive and } \\
\text { basement rocks. }\end{array}$ & Stable crust & $\begin{array}{l}\text { Generally weakly magnetic flat lying strata over } \\
\text { magnetic basement. Northeast magnetic } \\
\text { lineaments correlate with major Precambrian fault } \\
\text { systems. West-trending anomalies occur over } \\
\text { northeast UT are due to Precambrian basement } \\
\text { and perhaps magnetic intrusive rocks. Crustal } \\
\text { thickness is on the order of } 35-45 \mathrm{~km} \text {. } \\
\text { Characterized by moderate to low heat flow. } \\
\text { Ringed by high heat flow. In general, } \\
\text { characterized by low seismicity except at its } \\
\text { margins. }\end{array}$ & $\begin{array}{l}\text { Smith, 1978; } \\
\text { Blackwell, } 1978 \\
\end{array}$ \\
\hline $\begin{array}{l}\text { Columbia } \\
\text { Plateaus }\end{array}$ & $\begin{array}{l}\text { Characteristics include: rolling plateau } \\
\text { with young incised valleys; complex } \\
\text { mountains and dissected volcanic } \\
\text { plateaus; young lava plateau with } \\
\text { immature drainage. }\end{array}$ & $\begin{array}{l}\text { Mostly extensive flat-lying volcanics } \\
\text { associated with the Columbia River } \\
\text { basalts and related rocks. Flood } \\
\text { basaltic volcanic setting. }\end{array}$ & $\begin{array}{l}\text { Generally low gravity relief } \\
\text { reflecting some NE-trending } \\
\text { fabric }\end{array}$ & $\begin{array}{l}\text { Dominated by high amplitude } \\
\text { and high frequency anomalies. }\end{array}$ & $\begin{array}{l}\text { Magnetic } \\
\text { volcanic rocks. }\end{array}$ & \begin{tabular}{|l} 
Extension, \\
Hotspot
\end{tabular} & $\begin{array}{l}\text { Crustal thickness is on the order of } 30-35 \mathrm{~km}, \\
\text { thinning to the north. Characterized by very low } \\
\text { seismicity and high to moderate heat flow. }\end{array}$ & $\begin{array}{l}\text { Smith, 1978; } \\
\text { Blackwell, } 1978\end{array}$ \\
\hline Great Basin & $\begin{array}{l}\text { Part of the Basin and Range Province o } \\
\text { Fenneman (1931). Consists of isolated } \\
\text { NNE-trending ranges (dissected block } \\
\text { mountains) separated by aggraded } \\
\text { desert plains of about equal width. }\end{array}$ & $\begin{array}{l}\text { Eastern part is mostly Paleozoic } \\
\text { stratified rocks overlain by Cenozoic } \\
\text { volcanogenic rocks. Western part } \\
\text { include Paleozoic and Mesozoic } \\
\text { stratified rocks overlain by Cenozoic } \\
\text { volcanogenic rocks. Central part is } \\
\text { characterized by many Cenozoic } \\
\text { calderas. }\end{array}$ & $\begin{array}{l}\text { Highly variable geophysical } \\
\text { character. Several large gravity } \\
\text { domains. Broadest scale } \\
\text { character reflects physiographic } \\
\text { basin. Northern Great Basin } \\
\text { corresponds to high gravity and } \\
\text { the southern to generally } \\
\text { moderate to low gravity. }\end{array}$ & $\begin{array}{l}\text { Highly variable geophysical } \\
\text { character. The western Great } \\
\text { Basin, over the Walker Lane, } \\
\text { consists of numerous high } \\
\text { frequency and high amplitude } \\
\text { anomalies displaying a dominant } \\
\text { NW-trending fabric. The central } \\
\text { Great Basin is punctuated by } \\
\text { several NNW-trending narrow } \\
\text { magnetic highs. Most of the } \\
\text { Great Basin is generally } \\
\text { characterized by low magnetic } \\
\text { relief (termed "quiet basement } \\
\text { zone" by Mabey et al., } 1978 .\end{array}$ & $\begin{array}{l}\text { Magnetic } \\
\text { extrusive, } \\
\text { intrusive and } \\
\text { basement rocks. }\end{array}$ & Extension & $\begin{array}{l}\text { Subdued magnetic expression in the GB occurs } \\
\text { over basement (Mabey et al., 1978). Many } \\
\text { anomalies may be associated with Phanerozoic } \\
\text { rocks (in strong contrast to abundant basement } \\
\text { anomalies over the Colorado Plateaus). Crustal } \\
\text { thickness is moderately low (25km) and } \\
\text { surrounded by significantly thicker crust in the } \\
\text { Sierra Nevada, Colorado Plateaus, and Snake } \\
\text { River Plain. Characterized by moderately low } \\
\text { seismicity except at its borders. An exception is } \\
\text { the Central Nevada Seismic Belt that runs north- } \\
\text { south trending narrow seismic zone aligned with } \\
\text { Dixie Valley. Characterized by moderate heat } \\
\text { flow. High heat flow observed in the northern } \\
\text { Great Basin (Battle Mountain High) and low heat } \\
\text { flow observed in the south (Eureka Low). }\end{array}$ & $\begin{array}{l}\text { Smith, 1978; } \\
\text { Blackwell, 1978; } \\
\text { Mabey and others, } 1978 \\
\end{array}$ \\
\hline Great Valley & $\begin{array}{l}\text { Part of the Pacific Border Province of } \\
\text { Fenneman (1931). Low fluviatile plain. }\end{array}$ & $\begin{array}{l}\text { North-south-trending, deep alluvial } \\
\text { basin filled with Cenozoic to recent } \\
\text { sediments. }\end{array}$ & $\begin{array}{l}\text { Characterized mostly by long } \\
\text { linear NNW-trending gravity } \\
\text { low. }\end{array}$ & $\begin{array}{l}\text { Characterized mostly by long } \\
\text { linear NNW-trending magnetic } \\
\text { high. }\end{array}$ & $\begin{array}{l}\text { Magnetic } \\
\text { obducted oceanic } \\
\text { crust. Low } \\
\text { density basin fill. }\end{array}$ & $\begin{array}{l}\text { Subduction, } \\
\text { Accretion }\end{array}$ & $\begin{array}{l}\text { Characterized by low heat flow, and bound by } \\
\text { high heat flow in the Sierra Nevada and Coast } \\
\text { Ranges. Crustal thickness is on the order of 20- } \\
\text { 30km. Characterized by low seismicity and is } \\
\text { bound by high seismicity in the Coast Ranges and } \\
\text { Sierra Nevada. }\end{array}$ & Hinze and Zietz, 1985 \\
\hline
\end{tabular}


Table 2. Physiograpic provinces and their geologic and geophysical character.

\begin{tabular}{|c|c|c|c|c|c|c|c|c|}
\hline $\begin{array}{c}\text { Physiographic } \\
\text { Province }\end{array}$ & Physiographic Description & Geologic Description & Gravity expression & Magnetic expression & $\begin{array}{c}\text { Generalized } \\
\text { geophysical } \\
\text { sources } \\
\end{array}$ & $\begin{array}{l}\text { General } \\
\text { tectonic } \\
\text { setting } \\
\end{array}$ & Notes & References \\
\hline Klamath Mtns & $\begin{array}{l}\text { Part of the Pacific Border Province of } \\
\text { Fenneman (1931). Uplifted and } \\
\text { dissected peneplain on resistant rocks. }\end{array}$ & $\begin{array}{l}\text { Highly thrusted and folded } \\
\text { metamorphic Mesozoic sedimentary } \\
\text { and intrusive rocks. }\end{array}$ & $\begin{array}{l}\text { Largely characterized by a large } \\
\text { prominent gravity high. }\end{array}$ & $\begin{array}{l}\text { Moderate magnetic high region } \\
\text { containing a few prominent } \\
\text { NNW-trending prominent highs. }\end{array}$ & \begin{tabular}{|l|} 
Complex of \\
Mesozoic \\
intrusive rocks, \\
Paleozoic and \\
Mesozoic \\
metasedimentary \\
rocks, and \\
ultramafic rocks \\
that are part of \\
the Klamath \\
Mountains.
\end{tabular} & $\begin{array}{l}\text { Accretion, } \\
\text { Batholith } \\
\end{array}$ & $\begin{array}{l}\text { Characterized by low heat flow and low } \\
\text { seismicity. Crustal thickness is on the order of } 30- \\
35 \mathrm{~km} \text {. }\end{array}$ & \\
\hline $\begin{array}{l}\text { Middle Rocky } \\
\text { Mtns }\end{array}$ & $\begin{array}{l}\text { Complex mountains, mainly anticlinal } \\
\text { ranges, intermontane basins. }\end{array}$ & \begin{tabular}{|l|} 
Includes folded and faulted Paleozoic \\
and Mesozoic stratified rocks on \\
Precambrian basement. Contains \\
Wyoming basin filled with Cenozoic \\
and Recent sedimentary rocks.
\end{tabular} & $\begin{array}{l}\text { Characterized by several } \\
\text { intermediate-sized gravity highs } \\
\text { and lows. Most prominent are } \\
\text { the NW-trending Wind River and } \\
\text { the Uinta Mountains anomalies. }\end{array}$ & $\begin{array}{l}\text { Characterized by moderate } \\
\text { magnetic fields and a few } \\
\text { magnetic highs and lows. } \\
\text { Prominent highs include the NW- } \\
\text { trending Wind River anomaly. }\end{array}$ & $\begin{array}{l}\text { Precambrian } \\
\text { basement and } \\
\text { basin fill. }\end{array}$ & Compression & $\begin{array}{l}\text { Crustal thickness is on the order of } 40 \mathrm{~km} \text {. Area } \\
\text { displays moderate to high heat flow and low } \\
\text { seismicity. }\end{array}$ & $\begin{array}{l}\text { Mabey and others, 1978; } \\
\text { Smith, } 1978\end{array}$ \\
\hline Mojave Desert & $\begin{array}{l}\text { Part of the Basin and Range Province of } \\
\text { Fenneman (1931). Consists of short } \\
\text { ranges in desert plains. }\end{array}$ & $\begin{array}{l}\text { Isolated faulted ranges formed in an } \\
\text { extensional transcurrent setting } \\
\text { associated with Great Basin, San } \\
\text { Andreas and Garlock Faults. Includes } \\
\text { Mesozoic plutonic rocks and varying } \\
\text { amounts of Precambrian through } \\
\text { Mesozoic stratified rocks. Deep } \\
\text { alluvial basins. }\end{array}$ & $\begin{array}{l}\text { Characterized by generally high } \\
\text { gravity values. }\end{array}$ & $\begin{array}{l}\text { Characterized by generally high } \\
\text { magnetic values. }\end{array}$ & Plutonic rocks. & $\begin{array}{l}\text { Batholith, } \\
\text { Extension }\end{array}$ & $\begin{array}{l}\text { Characterized by moderate to low seismicity and } \\
\text { is bound by high seismicity associated with the } \\
\text { San Andreas and Garlock Fault systems. Heat } \\
\text { flow is moderate to high. Crustal thickness is on } \\
\text { the order of } 20-30 \mathrm{~km} \text {. }\end{array}$ & $\begin{array}{l}\text { Smith, 1978; } \\
\text { Blackwell, } 1978\end{array}$ \\
\hline $\begin{array}{l}\text { Northern Rocky } \\
\text { Mtns }\end{array}$ & Deeply dissected mountain uplands. & $\begin{array}{l}\text { Central and western parts consist of } \\
\text { Idaho Batholith and Challis } \\
\text { volcanics. Central and eastern parts } \\
\text { consist of crystalline Precambrian } \\
\text { rocks and Paleozoic strata. } \\
\text { Batholithic and high level silicic } \\
\text { volcanic activity. Abundant listric } \\
\text { thrust faults. }\end{array}$ & $\begin{array}{l}\text { Western half is characterized by } \\
\text { low gravity values. Eastern half } \\
\text { is characterized by high gravity. }\end{array}$ & $\begin{array}{l}\text { Relatively low magnetic relief, } \\
\text { though punctuated by some } \\
\text { moderate magnetic highs. }\end{array}$ & $\begin{array}{l}\text { Low density and } \\
\text { magnetic } \\
\text { intrusive rocks of } \\
\text { Idaho batholith } \\
\text { and Precambrian } \\
\text { and Paleozoic } \\
\text { strata. }\end{array}$ & $\begin{array}{l}\text { Batholith, } \\
\text { Extension }\end{array}$ & $\begin{array}{l}\text { Crustal thickness is on the order of } 35-40 \mathrm{~km} \text {. } \\
\text { Characterized by low seismicity and moderate to } \\
\text { low heat flow. }\end{array}$ & $\begin{array}{l}\text { Smith, 1978; } \\
\text { Blackwell, 1978 }\end{array}$ \\
\hline Sierra Nevada & $\begin{array}{l}\text { Part of the Cascade-Sierra Mountain } \\
\text { chain of Fenneman (1931). Block } \\
\text { mountain range tilted west. Accordant } \\
\text { crests. Alpine peaks near east side. }\end{array}$ & $\begin{array}{l}\text { Mesozoic batholithic complex locally } \\
\text { capped by Cenozoic volcanic rocks } \\
\text { and bounded by high angle normal } \\
\text { fault on the east. Intruded into belts } \\
\text { of accreted terranes that crop out } \\
\text { extensively in the western part of the } \\
\text { range. }\end{array}$ & $\begin{array}{l}\text { Characterized by several large } \\
\text { prominent NW-trending gravity } \\
\text { highs and lows. }\end{array}$ & $\begin{array}{l}\text { Characterized by several large } \\
\text { prominent NW-trending } \\
\text { magnetic highs and lows. }\end{array}$ & $\begin{array}{l}\text { Plutonic, } \\
\text { volcanic, } \\
\text { ophiolite rocks. }\end{array}$ & $\begin{array}{l}\text { Batholith, } \\
\text { Accretion }\end{array}$ & $\begin{array}{l}\text { Crustal thickness is up to } 50 \mathrm{~km} \text {. Displays high } \\
\text { heat flow. Characterized by high seismicity at its } \\
\text { eastern margin. }\end{array}$ & $\begin{array}{l}\text { Hinze and Zietz, 1985; } \\
\text { Oliver, 1977; } \\
\text { Smith, 1978; } \\
\text { Blackwell, 1978; } \\
\text { Lachenbruch and Sass, } 1978\end{array}$ \\
\hline Snake River Plain & $\begin{array}{l}\text { Part of the Columbia Plateaus of } \\
\text { Fenneman (1931). Young lava plateau } \\
\text { that features recent volcanism and } \\
\text { ineffective drainage. }\end{array}$ & $\begin{array}{l}\text { Mostly flat-lying flows and cinder } \\
\text { cones and associated poorly lithified } \\
\text { Micocene and younger sedimentary } \\
\text { rocks that cover a series of Miocene } \\
\text { and younger silicic calderas. } \\
\text { Considered to mark the trace of the } \\
\text { Yellowstone hotspot. }\end{array}$ & $\begin{array}{l}\text { Characterized by prominent } \\
\text { gravity high over entire Province. }\end{array}$ & $\begin{array}{l}\text { Characterized by moderately } \\
\text { high magnetic fields punctuated } \\
\text { by several small, prominent } \\
\text { magnetic highs. }\end{array}$ & Volcanic rocks. & Hotspot & $\begin{array}{l}\text { Crustal thickness is on the order of } 30-40 \mathrm{~km} \text {. } \\
\text { Displays moderate heat flow and low seismicity to } \\
\text { the west increasing to high heat flow and active } \\
\text { seismicity to the east. }\end{array}$ & $\begin{array}{l}\text { Smith, 1978; } \\
\text { Blackwell, 1978; } \\
\text { Mabey and others, 1978; } \\
\text { Eaton and others, 1978. }\end{array}$ \\
\hline
\end{tabular}


Province Great Basin

Tectonic Setting extension

\section{Terrane Definition}

Regional long-wavelength gravity anomaly spans entire GB and has bi-lateral symmetry (resembling the form of a butterfly). Anomaly appears best in isostatic gravity and topography, but expressed also in pseudogravity (see regional isostatic field map, figure 4). Corresponds with regional magnetic low.

Hi/lo/both

\section{Geologic Province}

Anomaly corresponds roughly to a topographically high region of extended crust associated with the GB Province.

\section{Inferred Source of Anomaly}

Anomaly reflects regionally extended, hot and elevated crust.

\section{Generalized source rock varied}

\section{References}

Fenneman, 1931;

Eaton and others, 1978;

Mabey and others, 1978;

Glen and others, 2000 


\section{Province Great Basin}

Tectonic Setting extension

\section{Terrane Definition}

Broadest regional pseudogravity feature corresponding roughly with the extent of the GB defined by topography (see regional pseudogravity map, figure 4). Corresponds with regional isostatic gravity low.

\section{Hi/lo/both}

\section{Geologic Province}

Anomaly corresponds roughly to a topographically high region of extended crust associated with the GB Province.

\section{Inferred Source of Anomaly}

Anomaly reflects regionally extended, hot and elevated crust.

\section{Generalized source rock varied}

References

Fenneman, 1931;

Eaton and others, 1978;

Mabey and others, 1978;

Glen and others, 2000 


\section{AZg01}

Scale I

Province Colorado Plateaus

Tectonic Setting uplift, stable crust, extension

Terrane Definition

Oval gravity high $(35 \mathrm{~km})$ that correlates with magnetic anomaly UTm20.

Hi/lo/both H

\section{Geologic Province}

Located on CP close to Hurricane Fault zone. Corresponds in part with Q and T volcanic centers.

\section{Inferred Source of Anomaly}

Source is buried by $\mathrm{Pz}$ and $\mathrm{Mz}$ strata. May be dense and magnetic buried $\mathrm{pC}$ basement or $\mathrm{Q}$ and $\mathrm{T}$ volcanic rocks.

Generalized source rock basement, volcanic mafic

References 


\section{AZg02}

Scale I

Province Colorado Plateaus

Tectonic Setting uplift, stable crust

Terrane Definition

Oval gravity high $(35 \mathrm{~km})$ over north end of magnetic anomaly AZm01.

Hi/lo/both H

\section{Geologic Province}

Located over a broader high on Kanab Plateau.

Inferred Source of Anomaly

Source is buried by $\mathrm{Pz}$ and $\mathrm{Mz}$ strata. Source may be dense and magnetic buried $\mathrm{pC}$ basement.

Generalized source rock basement

References 


\section{AZg03 \\ Scale I}

Province Colorado Plateaus

Tectonic Setting uplift

Terrane Definition

EW-elongate regional gravity high $(250 \times 125 \mathrm{~km})$ containing some prominent large $(\sim 100 \mathrm{~km}$ diameter $)$ highs.

Hi/lo/both H

\section{Geologic Province}

Northern part of feature corresponds to CP/GB transition. Western part is entirely within the GB. Northern and western portions correspond closely with outcrops of Pz strata.

Inferred Source of Anomaly

Shallow pC-basement rocks.

Generalized source rock basement

References 
AZg04

Scale I

Province Colorado Plateaus

Tectonic Setting uplift, stable crust

Terrane Definition

Broad gravity high (350x150km).

Hi/lo/both H

\section{Geologic Province}

Located over $\mathrm{Pz}$, and thickest $\mathrm{Mz}$ strata in AZ. coincides in part with the Black Mesa Basin.

Inferred Source of Anomaly

Regionally elevated dense pC basement rocks beneath CP.

Generalized source rock basement

References

Sumner, 1985 


\section{AZg05}

Province Colorado Plateaus

Tectonic Setting uplift, stable crust

\section{Terrane Definition}

NE-trending gravity high (50x25km). Edges correspond to steep magnetic gradients.

\section{Hi/lo/both H}

\section{Geologic Province}

Located over T and Q volcanic rocks of the San Francisco Peaks volcanic field.

Trend is similar to axes of monoclines in Mz strata that may indicate step faults in basement.

\section{Inferred Source of Anomaly}

Uplifted dense $\mathrm{pC}$ basement or dense intrusive rocks associated with $\mathrm{Cz}$ volcanic field.

Generalized source rock basement, intrusive mafic

References 


\section{AZg06}

Scale I

Province Colorado Plateaus

Tectonic Setting uplift

Terrane Definition

NE-trending gravity low $(100 \times 25 \mathrm{~km})$ within a much broader gravity low. Corresponds with magnetic low.

Hi/lo/both L

\section{Geologic Province}

Lies within the transition zone between GB CP within a broader gravity low, over $\mathrm{pC}$ basement and $\mathrm{Mz}$ granitic rocks.

\section{Inferred Source of Anomaly}

Low density, non-magnetic $\mathrm{pC}$ basement (silicic gneisses) and/or Mz granitic rocks.

Generalized source rock basement, intrusive silicic

References

Sumner, 1985 
AZg07

Scale I

Province Colorado Plateaus

Tectonic Setting uplift

Terrane Definition

Prominant NW-trending gravity high $(175 \times 50 \mathrm{~km})$. Corresponds closely with a magnetic low (not numbered).

Hi/lo/both H

Geologic Province

Located near the south edge of CP (Mogollon Rim).

Inferred Source of Anomaly

Dense, nonmagnetic crystalline pC-basement.

Generalized source rock basement

References 


\section{AZm01 \\ Scale I}

Province Colorado Plateaus

Tectonic Setting uplift, stable crust

Terrane Definition

NW-trending magnetic high $(75 \times 40 \mathrm{~km})$ within a broader high of $\mathrm{CP}$. North end corresponds to gravity high $\mathrm{AZg} 02$.

Hi/lo/both H

Geologic Province

Kaibab (Unikaret) Plateau (CP).

Inferred Source of Anomaly

Buried by $\mathrm{Pz}$ and $\mathrm{Mz}$ strata. Uplifted magnetic pC basement.

Generalized source rock basement

References 


\section{AZm02 \\ Scale I}

Province Colorado Plateaus

Tectonic Setting stable crust, uplift

Terrane Definition

NE-trending elongate magnetic high $(75 \times 25 \mathrm{~km})$ located within a much larger gravity low.

Hi/lo/both H

\section{Geologic Province}

Over Pz and Mz strata over the Paria Plateau (CP). Parallels plunging anticline, and is in line with the northeastern extension of the Bright Angle Fault.

Inferred Source of Anomaly

Magnetic intrusive or faulted and uplifted basement rocks.

Generalized source rock intrusive mafic, basement

References 


\section{AZm03 \\ Scale I}

Province Colorado Plateaus

Tectonic Setting uplift, stable crust

Terrane Definition

Prominent east-trending magnetic low $(75 \times 40 \mathrm{~km})$ that is within a much larger region of gravity high.

\section{Hi/lo/both L}

\section{Geologic Province}

Over flat-lying Pz and Mz strata of CP. Corresponds to the NW part of the Black Mesa Basin.

\section{Inferred Source of Anomaly}

Source is covered by $\mathrm{Pz}$ and $\mathrm{Mz}$ strata. Possible uplift of non-magnetic pC basement.

Generalized source rock basement

\section{References}

Sumner, 1985 


\section{AZm04 \\ Scale I}

Province Colorado Plateaus

Tectonic Setting uplift, stable crust

Terrane Definition

Equidimensional $(40 \mathrm{~km})$ magnetic high. Over a complex gravity anomaly. Mostly within but near the edge of a generally high magnetic region.

Hi/lo/both H

Geologic Province

At west edge of CP. Includes Iron Mtn volcanic rocks.

Inferred Source of Anomaly

Probable uplifted magnetic $\mathrm{pC}$ basement or $\mathrm{Cz}$ volcanic and associated intrusive rocks.

Generalized source rock basement, volcanic mafic, intrusive mafic

References 


\section{AZm05 \\ Scale I}

Province Colorado Plateaus

Tectonic Setting uplift

Terrane Definition

EW-trending elongate magnetic anomaly high $(250 \mathrm{~km})$ that narrows at its west end $(10 \mathrm{~km}$ wide extending for $\sim 150 \mathrm{~km}$ ). East end widens to $\sim 75 \mathrm{~km}$.

Hi/lo/both H

\section{Geologic Province}

East end includes a large part of Coconino plateau. West end roughly corresponds with trace of the Colorado River and Lake Mead. Includes Mt Floyd volcanic field.

\section{Inferred Source of Anomaly}

Probable uplifted magnetic $\mathrm{pC}$ basement uplifted on east-trending fault, or $\mathrm{Cz}$ volcanic or intrusive rocks.

Generalized source rock basement, intrusive silicic, volcanic silicic

\section{References}

Sumner, 1985 


\section{AZm06 \\ Scale I}

Province Colorado Plateaus

Tectonic Setting stable crust

Terrane Definition

NE-trending elongate magnetic high $(250 \mathrm{~km} \times 25 \mathrm{~km})$. SE-edge corresponds with gravity lineations. Includes three small anomalies at SW end.

Hi/lo/both H

\section{Geologic Province}

Southern part correlates with San Francisco Peaks volcanic field. Straddles edge of CP. Anomaly resembles that associated with the Springerville volcanic field to SE. Trend is parallel to the Colorado Lineament which may be related to the Colorado Mineral Belt located to the NE. Aligned along Mesa Butte Fault.

\section{Inferred Source of Anomaly}

Magnetic pC basement or $\mathrm{Cz}$ igneous rocks that exploited a fracture system in the basement.

Generalized source rock basement, intrusive mafic, volcanic mafic

References

Stewart and Crowell, 1992 


\section{AZm07 \\ Scale I}

Province Colorado Plateaus

Tectonic Setting extension

Terrane Definition

NE-trending elongate magnetic high $(100 \times 25 \mathrm{~km})$. Corresponds with NE-trending linear gravity features.

Hi/lo/both H

\section{Geologic Province}

Located in transition zone of CP and BR, over Hackberry Miocene lava flows.

Inferred Source of Anomaly

Magnetic volcanic rocks and feeder dikes.

Generalized source rock volcanic mafic

References 


\section{AZm08 \\ Scale I}

Province Colorado Plateaus

Tectonic Setting uplift

Terrane Definition

NE-trending elongate magnetic high $(100 \times 25 \mathrm{~km})$ located on a gravity low.

Hi/lo/both H

Geologic Province

Located in transition zone of $\mathrm{CP}$ and $\mathrm{BR}$, on pC (Apache group) rocks.

Inferred Source of Anomaly

Magnetic pC basement.

Generalized source rock basement

References 


\section{CAg01 \\ Scale I}

Province Klamath Mtns

Tectonic Setting accretion

Terrane Definition

Narrow roughly NS-trending gravity high $(100 \times 10 \mathrm{~km})$. Located entirely within a magnetic high.

Hi/lo/both $\mathrm{H}$

\section{Geologic Province}

Anomaly located over complex of $\mathrm{Mz}$ intrusive rocks, $\mathrm{Pz}$ and $\mathrm{Mz}$ metasedimentary rocks, and ultramafic rocks that are part of the Klamath Mtns. Gravity anomaly is centered over a magnetic high (CAm01).

\section{Inferred Source of Anomaly}

Gravity anomaly mimics crescent-shaped structural slivers, and is probably due to a dense portion of the eastern Klamath Mtns, probably mafic ultramafic rocks of the Trinity Ophiolite.

Generalized source rock intrusive mafic, volcanic mafic, ultramafic

References

LaFehr, 1966;

Griscom,1980b;

Jachens and Griscom, 1985;

Jachens and others, 1989 


\section{CAg02 \\ Scale I}

Province Cascade Mtns

Tectonic Setting accretion

Terrane Definition

Oval gravity low $(75 \mathrm{~km})$ within regionally complex magnetic region (CAm02).

\section{Hi/lo/both L}

\section{Geologic Province}

Over $\mathrm{Q}$ and $\mathrm{T}$ volcanic arc terrain. Includes Mount Shasta at its SW end. It is ringed on its southwest half by Klamath Mtns Mz intrusive rocks.

Inferred Source of Anomaly

Probably thick sequence of low density volcanic rocks.

Generalized source rock volcanic silicic

References

Griscom,1980a 


\section{CAg03 \\ Scale I}

Province Klamath Mtns

Tectonic Setting accretion

Terrane Definition

$\mathrm{NW}$-elongate gravity high $(50 \times 20 \mathrm{~km})$. Corresponds to a magnetic high region.

Hi/lo/both H

Geologic Province

Primarily on Pz limestones of the Klamath Mtns.

Inferred Source of Anomaly

Probably due to dense, magnetic mafic and ultramafic rocks of the Trinity

Ophiolite, though some of gravity anomaly may be due to dense carbonate rocks.

Generalized source rock ultramafic, sedimentary carbonate

References 


\section{CAg04 \\ Scale I}

Province Sierra Nevada

Tectonic Setting accretion

Terrane Definition

NW-trending moderate gravity high (30x20km).

Hi/lo/both H

\section{Geologic Province}

Over $\mathrm{Q}$ and $\mathrm{T}$ volcanic rocks.

Inferred Source of Anomaly

Probably dense, magnetic metamorphic or intrusive rocks of the Sierra Nevada foothills and Klamath Mtns.

Generalized source rock intrusive mafic, metamorphic mafic

References 


\section{CAg05}

\section{Scale}

\section{Province Great Basin}

Tectonic Setting batholith, extension

\section{Terrane Definition}

NW-trending gravity high $(75 \times 50 \mathrm{~km})$. Located over a prominant magnetic high.

Feature looks similar (in gravity and magnetics) to feature (CAg07).

Hi/lo/both H

Geologic Province

Over T volcanic rocks.

Inferred Source of Anomaly

Due to relatively dense and magnetic $\mathrm{Mz}$ granitic T volcanic rocks similar to feature CAg7.

Generalized source rock volcanic mafic, intrusive silicic

References 


\section{CAg06 \\ Scale I}

Province Sierra Nevada

Tectonic Setting accretion

Terrane Definition

NW-trending moderate gravity high $(100 \times 20 \mathrm{~km})$. Located mostly over a magnetic low (unlabeled).

Hi/lo/both H

\section{Geologic Province}

Over $\mathrm{Mz}$ and Pz rocks of the western foothills of the Sierra Nevada.

\section{Inferred Source of Anomaly}

Sources are various $\mathrm{NW}$-trending $\mathrm{Mz}$ and $\mathrm{Pz}$ intrusive and metamorphic rocks in western Sierra Nevada foothills. Anomaly likely due to intrusive and metamorphic rocks.

Generalized source rock intrusive mafic, metamorphic

References

Burnett and Jennings, 1982 


\section{CAg07 \\ Scale I}

Province Great Basin

Tectonic Setting batholith, extension

Terrane Definition

NW-trending (100x50km) variable gravity high. Located over a magnetic high region. Gravity anomaly is similar to the main Sierra Nevada batholith CAg12, though more magnetic.

Hi/lo/both H

\section{Geologic Province}

Over T volcanic rocks, $\mathrm{Mz}$ granitic and metavolcanic rocks, and some $\mathrm{Mz}$ and $\mathrm{Pz}$ strata in the NW part of anomaly.

\section{Inferred Source of Anomaly}

Due to relatively dense and magnetic Mz granitic or metavolcanic rocks, or $\mathrm{T}$ volcanic rocks.

Generalized source rock volcanic mafic, intrusive silicic

References 


\section{CAg08 \\ Scale I}

Province Great Valley

Tectonic Setting accretion

\section{Terrane Definition}

NW-trending prominent gravity high (150x50km). Forms northern end of prominent magnetic high (CAm19).

Hi/lo/both H

Geologic Province

Entirely over north end of Great Valley. Sutter Buttes lies at its center.

Inferred Source of Anomaly

May be dense, magnetic mafic volcanic roots, obducted magnetic oceanic crust or ultramafic rocks (ophiolite).

Generalized source rock intrusive mafic, volcanic mafic, ultramafic

\section{References}

Griscom 1973;

Cady, 1975;

Jachens and others, 1989;

Jachens and others, 1995 


\section{CAg09}

\section{Scale}

Province Sierra Nevada

Tectonic Setting accretion, batholith

\section{Terrane Definition}

NS-trending prominent gravity high $(40 \times 25 \mathrm{~km})$. Located within gravity anomaly CAg10, and magnetic anomaly high CAm11.

Hi/lo/both H

\section{Geologic Province}

Largely over Mz granitic pluton, and some Pz carbonates.

\section{Inferred Source of Anomaly}

May be due to dense, magnetic intrusive rocks or perhaps dense, magnetic, mafic and ultramafic oceanic crust (Smartville Ophiolite).

Generalized source rock intrusive mafic, ultramafic

References

Oliver, 1980 


\section{CAg10 \\ Scale I}

Province Sierra Nevada

Tectonic Setting accretion, batholith

\section{Terrane Definition}

Prominent NW-trending gravity high (100x40km).

Hi/lo/both H

\section{Geologic Province}

Over Mz granitic plutons, Pz carbonate and ultramafic rocks.

\section{Inferred Source of Anomaly}

May be due to dense, magnetic intrusive rocks or dense, magnetic, mafic and ultramafic thrust slices in Klamath Mtns and Sierra Nevada.

Generalized source rock intrusive mafic, ultramafic

\begin{tabular}{l}
\hline References \\
LaFehr, 1966; \\
Jachens and Griscom, 1986; \\
Jachens and others, 1989 \\
\\
\hline
\end{tabular}




\section{CAg11 \\ Scale I}

Province Great Valley, Sierra Nevada

Tectonic Setting batholith

Terrane Definition

Oval gravity low $(50 \mathrm{~km})$.

Hi/lo/both L

Geologic Province

Over a Mz pluton.

Inferred Source of Anomaly

Low density intrusive rocks.

Generalized source rock intrusive silicic

References

Oliver, 1980 


\section{CAg12 \\ Scale I}

Province Sierra Nevada, Great Valley

Tectonic Setting accretion, batholith

Terrane Definition

NNW-trending gravity high (275x75km).

Hi/lo/both H

Geologic Province

Occurs over $\mathrm{Pz}$ and $\mathrm{Mz}$ meta-sedimentary, metavolcanic, oceanic crustal rocks (ophiolites), and granitic rocks.

Inferred Source of Anomaly

Dense mafic and ultramafic ophiolite rocks.

Generalized source rock intrusive mafic, volcanic mafic, ultramafic

References

Oliver, 1980 


\section{CAg13 \\ Scale I}

Province Coast Ranges

Tectonic Setting transcurrent, depression

Terrane Definition

Narrow NW-trending gravity low $(150 x<10 \mathrm{~km})$ in line with gravity low CAg24.

\section{Hi/lo/both L}

Geologic Province

Follows along trace of the Hayward and Rodgers Creek Faults.

Inferred Source of Anomaly

Low density valley fill in East Bay Trough, and eastern Santa Clara and Livermore Valleys.

Generalized source rock sedimentary siliceous

References

Roberts and Jachens, 1993 


\section{CAg14 \\ Scale I}

Province Great Valley, Sierra Nevada

Tectonic Setting accretion

Terrane Definition

NW-trending gravity high (50x20km).

Hi/lo/both H

\section{Geologic Province}

Mostly over alluvium and Tertiary sediments.

Inferred Source of Anomaly

May be due to dense mafic to ultramafic intrusive bodies or to dense metasedimentary rocks.

Generalized source rock intrusive mafic, ultramafic, metamorphic mafic

References 
CAg15

Scale I

Province Great Valley

Tectonic Setting depression

Terrane Definition

Prominent, elongate NW-trending gravity low $(700 \times 50 \mathrm{~km})$. Strong gradients on eastern edge and in parts on its western edge. Located over magnetic low anomaly CAm14.

\section{Hi/lo/both L}

\section{Geologic Province}

Located at westernmost edge of Great Valley.

Inferred Source of Anomaly

Likely source is low density valley fill.

Generalized source rock sedimentary siliceous

References

Byerly, 1966;

Suppe, 1979;

Oliver and Griscom, 1980;

Jachens and others, 1989 


\section{CAg16}

Scale $R$

Province Sierra Nevada

Tectonic Setting batholith

Terrane Definition

Prominent NW-trending gravity low $(550 \times 75 \mathrm{~km})$. Corresponds with magnetic high anomaly CAm17.

\section{Hi/lo/both L}

\section{Geologic Province}

Gravity anomaly is broader than the corresponding magnetic anomaly (CAm17), but also located mostly over eastern Sierra Nevada batholith.

Inferred Source of Anomaly

Magnetic, low density granitic rocks.

Generalized source rock intrusive silicic

References

Oliver and Mabey, 1963;

Griscom and Oliver, 1980;

Jachens and others, 1989 


\section{CAg17 \\ Scale I}

Province Coast Ranges

Tectonic Setting transcurrent, accretion

Terrane Definition

Prominent narrow NW-trending gravity high $(>100 \times 5 \mathrm{~km})$. Southern half is located over magnetic high CAm21.

Hi/lo/both $\mathrm{H}$

Geologic Province

Over Franciscan Complex rocks.

Inferred Source of Anomaly

May be due to mafic and ultramafic rocks of the Franciscan Complex, such as greenstones of Permanente and Headlands terranes.

Generalized source rock extrusive mafic, intrusive mafic, ultramafic

References

Jachens and Griscom, in press 


\section{CAg18}

Scale $R$

Province Coast Ranges

Tectonic Setting transcurrent, accretion, depression, uplift

\section{Terrane Definition}

Irregular, NW-trending, long, linear, moderate gravity high (400x25km) defined by gravity lows to the east (CAg15) and to the west (CAm23,24,30)

Hi/lo/both B

\section{Geologic Province}

Eastern Coast Ranges. Varied moderately high-density rocks within the San Andreas Fault zone.

\section{Inferred Source of Anomaly}

Various sources whose alignment is associated with accretion along continental margin and with subsequent strike-slip faulting within the San Andreas Fault zone. Anomaly is due to exposed $\mathrm{Mz}$ bedrock surounded by low-density $\mathrm{Cz}$ rocks and basin fill.

Generalized source rock sedimentary siliceous, ultramafic, volcanic mafic 


\section{CAg19 \\ Scale L}

Province Sierra Nevada

Tectonic Setting accretion

Terrane Definition

Narrow linear NNW-trending gravity high (50x2km).

Hi/lo/both H

Geologic Province

Occurs over Pz and Mz metasedimentary, metavolcanic, ophiolite, and granitic rocks.

Inferred Source of Anomaly

Dense mafic and ultramafic ophiolite rocks

Generalized source rock intrusive mafic, volcanic mafic, ultramafic

References

Oliver, 1980 


\section{CAg20}

\section{Scale}

Province Coast Ranges

Tectonic Setting accretion, transcurrent

Terrane Definition

NW-trending gravity high (50x10km).

Hi/lo/both H

\section{Geologic Province}

Located over Mz Ben Lomond pluton in the Santa Cruz Mtns.

Inferred Source of Anomaly

Magnetic Logan Gabbro beneath Ben Lomond pluton.

Generalized source rock intrusive mafic

References

Jachens and others, 1998 


\section{CAg21 \\ Scale L}

Province Sierra Nevada, Great Valley

Tectonic Setting batholith

Terrane Definition

Oval gravity low $(50 \times 40 \mathrm{~km})$ associated with a magnetic low. Similar to other western Sierra gravity belt lows (e.g. CAg11, and unamed low south of anomaly CAg06).

Hi/lo/both L

Geologic Province

Occurs mainly over Mz granitic rocks and some Mz metasedimentary, and T,Q sedimentary rocks.

Inferred Source of Anomaly

Low density silicic plutons

Generalized source rock intrusive felsic

References

Oliver and others, 1992 


\section{CAg22 \\ Scale I}

Province Coast Ranges

Tectonic Setting transcurrent, accretion

Terrane Definition

Narrow NW-trending gravity high $(125 \mathrm{x}<10 \mathrm{~km})$.

Hi/lo/both H

Geologic Province

Over Salinian Mz intrusive body. Eastern edge is along San Andreas Fault.

Inferred Source of Anomaly

Relatively dense intrusive body surrounded by low-density basin fill.

Generalized source rock intrusive silicic

References 
CAg23

Scale

Province Coast Ranges

Tectonic Setting accretion, transcurrent

Terrane Definition

NW-trending gravity high (60x10km). Corresponds with a NW-trending elongate magnetic high.

Hi/lo/both H

\section{Geologic Province}

Located over eastern edge of Coast Range Franciscan complex - includes ultramafic rocks (serpentines and eclogites).

Inferred Source of Anomaly

Due to Franciscan metasedimentary rocks.

Generalized source rock ultramafic

References 


\section{CAg24 \\ Scale I}

Province Coast Ranges

Tectonic Setting transcurrent, depression

Terrane Definition

Narrow NW-trending gravity low $(125 \mathrm{x}<10 \mathrm{~km})$.

\section{Hi/lo/both L}

\section{Geologic Province}

Located over Coyote valley Q and T valley fill, along eastern side of San Andreas Fault.

Inferred Source of Anomaly

Low density valley fill.

Generalized source rock sedimentary siliceous

References 


\section{CAg25}

Scale I

Province Sierra Nevada, Great Valley

Tectonic Setting accretion, batholith

\section{Terrane Definition}

Large, NNW-trending moderate gravity high (600x150km) containing several prominant highs (e.g. CAg12, CAg26) and lows (e.g. CAg21)

Hi/lo/both $\mathrm{H}$

\section{Geologic Province}

Western part occurs over Great Valley sediments. Eastern part includes granitic rocks of the Sierra Nevada Batholith and $\mathrm{Mz}$ and Pz metasedimentary rocks of the western Sierra Nevada.

Inferred Source of Anomaly

Dense mafic and ultramafic ophiolite rocks

Generalized source rock intrusive mafic, volcanic mafic, ultramafic

References

Oliver and Griscom, 1980 


\section{CAg26 \\ Scale L}

Province Sierra Nevada, Great Valley

Tectonic Setting accretion

Terrane Definition

NNW-trending gravity high (150x70km). Anomaly occurs largely over a magnetic low.

Hi/lo/both H

Geologic Province

Western part of anomaly occurs over sediments of the Great Valley. Eastern edge occurs over Sierra Nevada Batholith and Mz and Pz metasedimentary rocks.

Inferred Source of Anomaly

Dense mafic and ultramafic ophiolite rocks.

Generalized source rock intrusive mafic, volcanic mafic, ultramafic

References

Oliver and Griscom, 1980 


\section{CAg27 \\ Scale L}

Province Sierra Nevada, Great Valley

Tectonic Setting accretion

Terrane Definition

NNW-trending narrow gravity high $(125 \times 20 \mathrm{~km})$ refered to as the Dinuba gravity lineament. Anomally occurs over a magnetic high.

Hi/lo/both H

\section{Geologic Province}

Occurs at contact between Great Valley and Sierra Nevada, mostly over mafic and ultramafic $\mathrm{Pz}$ and $\mathrm{Mz}$ oceanic crustal rocks.

Inferred Source of Anomaly

Dense mafic and ultramafic ophiolite rocks.

Generalized source rock intrusive mafic, volcanic mafic, ultramafic

References

Oliver, 1980 
CAg28

Scale I

Province Great Basin

Tectonic Setting uplift

Terrane Definition

Prominent oval gravity high $(50 \mathrm{~km})$ located over a magnetic low region.

Hi/lo/both H

Geologic Province

Located over the north end of Death Valley over pC crystalline basement.

Inferred Source of Anomaly

Dense crystalline basement.

Generalized source rock basement mafic

References

Chapman and others, 1973 


\section{CAg29 \\ Scale I}

Province Coast Ranges

Tectonic Setting transcurrent, accretion

Terrane Definition

NW-trending gravity high (100x50km).

Hi/lo/both H

\section{Geologic Province}

Located over Franciscan assemblage metamorphic (Sur Series) rocks to the west of the Hosgri Fault and K Salinian granites to the east.

Inferred Source of Anomaly

Salinian K granites, Franciscan (Sur series) metasedimentary and metavolcanic rocks.

Generalized source rock sedimentary siliceous, extrusive mafic, metamorphic mafic, intrusive silicic

References 


\section{CAg30 \\ Scale I}

Province Coast Ranges

Tectonic Setting transcurrent, depression

\section{Terrane Definition}

Prominent NW-trending gravity low $(>300 \times 25 \mathrm{~km})$. Along it western edge is a magnetic high (CAm31).

\section{Hi/lo/both L}

\section{Geologic Province}

Follows the Salinas Valley Q fill and some Miocene sedimentary rocks. Anomaly is bound on its western edge by Salinian granitic basement.

Inferred Source of Anomaly

Low density basin fill.

Generalized source rock sedimentary siliceous

References

Chapman and Griscom, 1980;

Jachens and others, 1989 


\section{CAg31}

Scale I

Province Great Basin

Tectonic Setting extension

Terrane Definition

Oval gravity high $(40 \mathrm{~km})$ located over a magnetic high at the southern end of Eastern Sierra magnetic anomaly (CAm34).

Hi/lo/both $\mathrm{H}$

\section{Geologic Province}

Located just north of the Garlock fault at the spillway between China and Searles Lakes. Located over Mz granitic rocks.

Inferred Source of Anomaly

Possibly dense, magnetic pluton.

Generalized source rock intrusive mafic

References 


\section{CAg32 \\ Scale I}

Province Great Basin, Mojave

Tectonic Setting extension

Terrane Definition

Prominent NW-trending gravity low $(100 \times 40 \mathrm{~km})$ located over a magnetic low region. A small oval magnetic high occurs at its north end.

Hi/lo/both L

Geologic Province

Located mostly over Mz Tectonia batholith, Mz Kingston Peak intrusive rocks.

Inferred Source of Anomaly

Low density intrusive body.

Generalized source rock intrusive silicic

References

Healey, 1970 


\section{CAg33}

\section{Scale}

Province Coast Ranges

Tectonic Setting depression, transcurrent

\section{Terrane Definition}

NW-trending gravity low ( $>50 \times 50 \mathrm{~km})$. Located immediately west of a similarly shaped moderate to low magnetic ridge.

Hi/lo/both L

Geologic Province

Located over T and K sediments of the Santa Maria Basin.

Inferred Source of Anomaly

Low density sedimentary rocks.

Generalized source rock sedimentary siliceous

References 


\section{CAg34}

\section{Scale}

Province Coast Ranges, Sierra Nevada, Great

Tectonic Setting batholith

Terrane Definition

Narrow ENE-trending gravity high (75×20km).

Hi/lo/both H

\section{Geologic Province}

Located just north of the Garlock Fault over Mz granitic rocks.

Inferred Source of Anomaly

Dense granitic rocks.

Generalized source rock intrusive silicic

References 


\section{CAg35}

Scale I

Province Great Basin, Mojave

Tectonic Setting transcurrent

Terrane Definition

ENE-trending linear feature $(\sim 100 \mathrm{~km})$ dividing a gravity high to the north and a low to the south. Approximately corresponds with trace of the Garlock Fault. Corresponds with magnetic anomaly CAm38.

Hi/lo/both B

\section{Geologic Province}

Left-lateral fault bounding southern edges of Coast Ranges, Great Valley, and Sierra Nevada with the Mojave desert.

Inferred Source of Anomaly

Edges of anomalies associated with Coast Ranges, Great Valley, Sierra Nevada, and Mojave Desert.

\section{Generalized source rock}

References

Davis and Burchfiel, 1973 


\section{CAm01 sale +}

Province Klamath Mtns

Tectonic Setting accretion

\section{Terrane Definition}

Irregular shaped prominent magnetic high (75 EW x 100km NS) that entirely encompasses the associated gravity anomaly (CAg01).

\section{Hi/lo/both H}

\section{Geologic Province}

Eastern lobe of magnetic anomaly corresponds with Mt Shasta. Bulk of magnetic anomaly located over complex of $\mathrm{Mz}$ intrusive rocks, $\mathrm{Pz}$ and $\mathrm{Mz}$ metasedimentary rocks, and ultramafic rocks that are part of the Klamath Mtns. Anomaly corresponds mostly with a gravity high (CAg01).

\section{Inferred Source of Anomaly}

Magnetic anomaly sources are varied. Mostly due to magnetic intrusive rocks of the Klamath Mtns. SE lobe of magnetic anomaly may be due to magnetic volcanic rocks of Mt Shasta.

Generalized source rock intrusive mafic, volcanic mafic, ultramafic

References

LaFehr, 1966;

Griscom,1980b;

Blakely and others, 1985;

Jachens and others, 1989 


\section{CAm02 sale n}

Province Columbia Plateaus

Tectonic Setting extension

\section{Terrane Definition}

Regional magnetic high consisting of several high frequency, high amplitude magnetic anomalies. Anomaly is continuous with feature ORm06.

Hi/lo/both $\mathrm{H}$

Geologic Province

Over T and Q andesite volcanic terrane, much of which is in Oregon.

Inferred Source of Anomaly

Magnetic volcanic rocks.

Generalized source rock volcanic mafic

References

Griscom, $1980 b$ 


\section{CAm03 sale +}

Province Columbia Plateaus, Cascade Mtns,

Tectonic Setting extension

\section{Terrane Definition}

Prominent kidney shaped NS-trending magnetic high $(60 \times 25 \mathrm{~km})$. Straddles moderate gravity high and low anomalies.

Hi/lo/both $\mathrm{H}$

\section{Geologic Province}

Located over Q volcanic rocks. Eastern edge butts up against Klamath series metavolcanic rocks.

Inferred Source of Anomaly

Magnetic volcanic rocks.

Generalized source rock volcanic mafic

References 


\section{CAm04 scate}

Province Sierra Nevada

Tectonic Setting accretion

\section{Terrane Definition}

Prominent NW-trending magnetic high (50x20km).

Hi/lo/both $\mathrm{H}$

Geologic Province

Over $\mathrm{Q}$ and $\mathrm{T}$ volcanic rocks.

\section{Inferred Source of Anomaly}

Probably dense, magnetic metamorphic or intrusive rocks of the Sierra Nevada foothills and Klamath Mtns.

Generalized source rock intrusive mafic, metamorphic mafic

$$
\text { References }
$$




\section{CAm05 sale I}

Province Sierra Nevada

Tectonic Setting accretion

\section{Terrane Definition}

Prominent NW-trending magnetic high (70x10km).

Hi/lo/both $\mathrm{H}$

Geologic Province

Over $\mathrm{Mz}$ and Pz rocks of the western foothills of the Sierra Nevada.

\section{Inferred Source of Anomaly}

Sources are various $\mathrm{NW}$-trending $\mathrm{Mz}$ and $\mathrm{Pz}$ intrusive and metamorphic rocks in western Sierra Nevada foothills. Anomaly likely due to magnetic intrusive and metamorphic rocks.

Generalized source rock intrusive mafic, metamorphic

References

Burnett and Jennings, 1982 


\section{CAm06 scate}

Province Sierra Nevada

Tectonic Setting accretion

\section{Terrane Definition}

Arcuate NNW-trending elongate magnetic low $(250 \times 25 \mathrm{~km}$, that broadens at southern edge to $100 \mathrm{~km}$ ).

\section{Hi/lo/both L}

\section{Geologic Province}

Shape of anomaly correlates well with outcrops of Pz marine sedimentary rocks consisting of limestones, sandstones, metavolcanics.

Inferred Source of Anomaly

Weakly magnetic sedimentary rocks and possibly serpentinites.

Generalized source rock sedimentary siliceous, ultramafic

$$
\text { References }
$$




\section{CAm07 seat}

\section{Province Great Basin}

Tectonic Setting batholith, extension

\section{Terrane Definition}

Prominent magnetic high $(175 \times 100 \mathrm{~km})$. Located over NW-trending gravity highs and lows (e.g. CAg05,07).

\section{Hi/lo/both H}

\section{Geologic Province}

Located over K granitic and T volcanic rocks. Eastern edge is aligned with magnetic feautre $\mathrm{NVm} 05$ and gravity feature $\mathrm{NVg} 01$.

\section{Inferred Source of Anomaly}

Due to magnetic granitic or volcanic rocks.

Generalized source rock intrusive silicic, volcanic mafic

$$
\text { References }
$$




\section{Province Sierra Nevada}

Tectonic Setting accretion

\section{Terrane Definition}

NNW-trending narrow magnetic zone $(225 \times 15 \mathrm{~km})$ with prominent eastern and western boundaries that separate it from a prominent high to the west (CAm19) and prominent low to the east (CAm09).

\section{Hi/lo/both B}

\section{Geologic Province}

Located over eastern Great Valley over Q sediments.

\section{Inferred Source of Anomaly}

Anomaly likely associated with anomaly CAm19 and represents magnetic obducted oceanic crust.

Generalized source rock ultramafic

References 


\section{CAm09 sate}

Province Sierra Nevada

Tectonic Setting accretion

\section{Terrane Definition}

NNW-trending elongate magnetic low (300x25km. Northern end broadens to $\sim 75 \mathrm{~km}$ ).

\section{Hi/lo/both L}

\section{Geologic Province}

Located over western edge of $\mathrm{Mz}$ and $\mathrm{Pz}$ metamorphic and granitic rocks of the western Sierra Nevada foothills.

\section{Inferred Source of Anomaly}

Weakly magnetic metamorphic rocks.

Generalized source rock intrusive silicic, metamorphic silicic, sedimentary siliceous

\section{References}




\section{CAm10 sate}

Province Sierra Nevada

Tectonic Setting accretion

\section{Terrane Definition}

Prominent narrow NS-trending magnetic high $(75 \times 5 \mathrm{~km})$. May be a continuation of anomaly CAm05.

Hi/lo/both $\mathrm{H}$

Geologic Province

Correlates with narrow body of ultramafic rocks.

Inferred Source of Anomaly

Strongly magnetic ultramafic rocks.

Generalized source rock ultramafic

References 


\section{CAm11}

Scale

Province Sierra Nevada

Tectonic Setting accretion, batholith

Terrane Definition

Large magnetic high $(110 \times 50 \mathrm{~km})$ surrounded by prominent lows.

Hi/lo/both H

\section{Geologic Province}

Located mostly over metavolcanic and metasedimentary rocks of Sierra Nevada foothill gold belt, and over remnants of Sierra Nevada intrusive rocks and some ultramafic bodies. Eastern edge correlates well with string of ultramafic rocks (Smartsville ophiolite).

Inferred Source of Anomaly

Magnetic metavolcanic and ultramafic rocks.

Generalized source rock volcanic mafic, intrusive mafic, ultramafic

References 


\section{CAm12 sale}

Province Coast Ranges

Tectonic Setting accretion, transcurrent

\section{Terrane Definition}

NW-trending magnetic high $(>75 \times 25 \mathrm{~km})$

Hi/lo/both $\mathrm{H}$

\section{Geologic Province}

Located over Franciscan assemblage and ultramafic rocks.

Inferred Source of Anomaly

Strongly magnetic ultramafic rocks of the Coast Range ophiolite that lie along the Coast Range Fault.

Generalized source rock ultramafic

References

Jachens and others, 1995 


\section{CAm13 \\ Scale}

Province Great Valley

Tectonic Setting accretion

Terrane Definition

Prominent NW-trending elongate magnetic high $(175 \times 20 \mathrm{~km})$. Anomaly splays off of feature CAm19.

Hi/lo/both $\mathrm{H}$

Geologic Province

Located at eastern edge of Great Valley over T and K sedimentary rocks.

Inferred Source of Anomaly

Likely source is strongly magnetic obducted oceanic crust.

Generalized source rock volcanic mafic, ultramafic

References

Griscom, 1966 


\section{CAm14 scat -}

Province Great Valley

Tectonic Setting depression

Terrane Definition

Elongate NW-trending magnetic low region directly west of prominent magnetic high (CAm19). Its western edge (less well defined) consists of a series of NW-trending discontinuous gradients. Located over gravity low anomaly CAg15.

Hi/lo/both L

Geologic Province

Located at westernmost edge of Great Valley.

Inferred Source of Anomaly

Likely source is weakly magnetic valley fill.

Generalized source rock sedimentary siliceous

References

Byerly, 1966;

Suppe, 1979;

Oliver and Griscom, 1980;

Jachens and others, 1989 


\section{CAm15}

Scale

Province Sierra Nevada

Tectonic Setting accretion

Terrane Definition

Oval magnetic high $(25 \times 20 \mathrm{~km})$.

Hi/lo/both H

\section{Geologic Province}

Located over Pz metamorphic, Q volcanic and ultramafic rocks along the Bear Mt Fault Zone.

Inferred Source of Anomaly

Likely due to strongly magnetic ultramafic rocks.

Generalized source rock ultramafic

References 


\section{CAm16 scate}

Province Sierra Nevada

Tectonic Setting accretion

\section{Terrane Definition}

Long NW-trending magnetic high (100x20km). Anomally is mostly associated with a much broader gravity high (CAg12).

Hi/lo/both $\mathrm{H}$

\section{Geologic Province}

Occurs over $\mathrm{Mz}$ and $\mathrm{Pz}$ metasedimentary rocks, granitic rocks, ophiolites and serpentinites along the Bear Mt fault zone and related faults.

\section{Inferred Source of Anomaly}

Dense mafic and ultramafic ophiolite rocks.

Generalized source rock intrusive mafic, ultramafic

References 


\section{CAm17 scale R}

Province Sierra Nevada

Tectonic Setting batholith

Terrane Definition

Prominent NW-trending magnetic high $(50 \times 400 \mathrm{~km})$.Corresponds with gravity low anomaly CAg16.

Hi/lo/both $\mathrm{H}$

\section{Geologic Province}

Magnetic anomaly located over eastern Sierra Nevada batholith. Gravity anomaly (CAg16) is broader, but also located mostly over eastern Sierra Nevada batholith.

Inferred Source of Anomaly

Magnetic, low density granitic rocks.

Generalized source rock intrusive silicic

References

Oliver and Mabey, 1963;

Griscom and Oliver, 1980;

Jachens and others, 1989 


\section{CAm18 scate +}

\section{Province Great Basin}

Tectonic Setting extension, batholith

\section{Terrane Definition}

Oblate EW trending moderate magnetic high (100x40km).

\section{Hi/lo/both H}

\section{Geologic Province}

Northeast of Long Valley Caldera, over Benton Range and north end of White Mtns, over $\mathrm{Q}$ volcanic and $\mathrm{Mz}$ intrusive rocks.

\section{Inferred Source of Anomaly}

Magnetic volcanic and intrusive rocks.

Generalized source rock volcanic mafic, intrusive mafic

\section{References}

Stewart and Carlson, 1978 


\section{CAm19 Scale R}

Province Great Valley

Tectonic Setting accretion

\section{Terrane Definition}

Prominent NW-trending magnetic high $(450 \times 40 \mathrm{~km})$. Strong gradients on its eastern edge and in parts on its western edge. Corresponds with subdued gravity high CAg25. Continuous with magnetic anomaly CAm26.

Hi/lo/both $\mathrm{H}$

\section{Geologic Province}

Located over basin fill of Great Valley. Anomaly trends parallel to Great Valley

Inferred Source of Anomaly

Slab of strongly magnetic obducted oceanic crust and mantle.

Generalized source rock volcanic mafic, ultramafic

\section{References}

Griscom, 1966;

Cady, 1975;

Griscom and Jachens, 1990;

Jachens and others, 1995 


\section{CAm20 sale -}

\section{Province Great Basin}

Tectonic Setting extension, batholith

\section{Terrane Definition}

Arcuate shaped magnetic high (75x10km).

\section{Hi/lo/both H}

\section{Geologic Province}

Located over Jurassic Cottonwood pluton. May be related to the Sylvania pluton which has same age and composition. A fault separates and may have offset these plutons.

\section{Inferred Source of Anomaly}

Magnetic intrusive rocks.

Generalized source rock intrusive mafic, intrusive silicic

References

McKee and Nelson, 1967 


\section{CAm21 \\ Scale}

Province Coast Ranges

Tectonic Setting transcurrent, accretion

Terrane Definition

NW-trending moderate magnetic high (150x25km). NW end corresponds with NW-trending elongate gravity high (CAg17). SE end corresponds with NW-trending gravity low (CAg24).

Hi/lo/both H

\section{Geologic Province}

Southern end follows the Santa Clara Valley, and is bound on the west by the San Andreas Fault. In the north, the anomaly crosses the San Andreas Fault and runs offshore.

\section{Inferred Source of Anomaly}

Anomaly is caused by 3 different sources in 3 different fault blocks. Western end is offshore, west of the San Gregorio Fault and is likely caused by Coast Range ophiolite rocks ocurring in Franciscan terrane. Central part is caused by deeply buried, concealed Logan gabbro of likely ophiolite origin. Southeastern part is due to slivers of Coast range ophiolite rocks in Franciscan terrane.

Generalized source rock intrusive mafic, ultramafic

References

Jachens and others, 1998 
Province Sierra Nevada, Great Valley

Tectonic Setting batholith, depression

\section{Terrane Definition}

Long NNW-trending magnetic low $(500 \times 75 \mathrm{~km})$ with internal magnetic highs (e.g. CAm27). Southern portion of anomaly crosses the Garlock Fault (CAm38) and is offset to the east by $\sim 75 \mathrm{~km}$.

\section{Hi/lo/both L}

\section{Geologic Province}

Western part of anomaly occurs over Great Valley sediments and $\mathrm{Mz}$ and $\mathrm{Pz}$ metasedimentary rocks. Eastern, central and southern parts occur over Sierra Nevada batholith. Southern most part of anomaly, south of the Garlock Fault, occurs over granitic rocks and valley fill of the Mojave Desert.

Inferred Source of Anomaly

Weakly magnetic sedimentary and granitic rocks.

Generalized source rock intrusive silicic, sedimentary siliceous

References 


\section{CAm23 sale -}

\section{Province Great Basin}

Tectonic Setting extension, batholith

\section{Terrane Definition}

Small WNW-trending moderate elongate magnetic high $(40 \times 10 \mathrm{~km})$ on the CA-NV border.

Hi/lo/both $\mathrm{H}$

Geologic Province

Located over Jurassic Sylvania pluton.

Inferred Source of Anomaly

Magnetic intrusive rocks.

Generalized source rock intrusive mafic, intrusive silicic

$$
\text { References }
$$

McKee, 1968 


\section{CAm24 seat}

\section{Province Great Basin}

Tectonic Setting extension, batholith

\section{Terrane Definition}

Elongate NW-trending magnetic high (60x10km).

\section{Hi/lo/both H}

\section{Geologic Province}

Located over Mz pluton in the Inyo Mtns.

Inferred Source of Anomaly

Magnetic intrusive rocks.

Generalized source rock intrusive mafic, intrusive silicic

$$
\text { References }
$$




\section{CAm25 \\ Scale}

Province Coast Ranges

Tectonic Setting transcurrent, accretion, batholith

Terrane Definition

NNW-trending magnetic high (60x20km).

\section{Hi/lo/both H}

\section{Geologic Province}

Located near the San Andreas Fault zone largely over T sedimentary and

Franciscan assemblage rocks. NE of the San Andreas Fault, the main part of anomaly is over a serpentinite diapir (New Idria Dome) and a largely concealed serpentinite, flat-lying body that extends SE to Table Mt. Southwest of the San Andreas Fault, at the south end of the anomaly, the anomaly is located over Cretaceous intrusive rocks.

\section{Inferred Source of Anomaly}

Serpentinites and magnetic intrusive rocks.

Generalized source rock ultramafic, intrusive mafic, intrusive silicic 


\section{CAm26 Scale R}

Province Great Valley

Tectonic Setting accretion

\section{Terrane Definition}

Prominent NW-trending magnetic high (200x50km). Corresponds with subdued gravity high CAg25. Continuous with magnetic anomaly CAm19.

Hi/lo/both $\mathrm{H}$

Geologic Province

Located over basin fill of Great Valley. Anomaly trends parallel to Great Valley

Inferred Source of Anomaly

Slab of strongly magnetic obducted oceanic crust and mantle.

Generalized source rock volcanic mafic, ultramafic

\section{References}

Cady, 1975;

Griscom and Jachens, 1990;

Jachens and others, 1995 


\section{CAm27 sate}

Province Sierra Nevada, Great Valley

Tectonic Setting accretion

\section{Terrane Definition}

Long linear NW-trending magnetic high $(125 \times 25 \mathrm{~km})$ corresponding to the Dinuba gravity high (CAg27).

Hi/lo/both $\mathrm{H}$

\section{Geologic Province}

Occurs at contact between Great Valley and Sierra Nevada, mostly over mafic and ultramafic $\mathrm{Pz}$ and $\mathrm{Mz}$ oceanic crustal rocks.

Inferred Source of Anomaly

Magnetic mafic and ultramafic ophiolite rocks.

Generalized source rock intrusive mafic, volcanic mafic, ultramafic

References 


\section{CAm28 \\ Scale}

Province Great Basin

Tectonic Setting extension, batholith

Terrane Definition

Prominent magnetic high $(40 \times 25 \mathrm{~km})$.

Hi/lo/both H

\section{Geologic Province}

Located over Mz pluton (Hunter Mt batholith) in the central Panamint Range.

Inferred Source of Anomaly

Magnetic intrusive rocks.

Generalized source rock intrusive mafic, intrusive silicic

References

Mabey, 1961;

Chen and Moore, 1982 


\section{CAm29 \\ Scale}

Province Great Valley

Tectonic Setting accretion

Terrane Definition

Small NW-trending magnetic high (10x20km).

Hi/lo/both $\mathrm{H}$

\section{Geologic Province}

Located over eastern Great Valley and within anomaly CAm26.

Inferred Source of Anomaly

May be a shallow sliver of obducted magnetic oceanic crust.

Generalized source rock volcanic mafic, intrusive mafic

References 


\section{CAm30 sate}

\section{Province Great Basin}

Tectonic Setting extension

\section{Terrane Definition}

Prominent elongate NW-trending magnetic high (70x25km).

\section{Hi/lo/both H}

\section{Geologic Province}

Located over T intrusive, volcanic, and possibly pC crystalline basement rocks in the Black Mtns.

\section{Inferred Source of Anomaly}

Likely due to magnetic intrusive, volcanic and/or basement rocks.

Generalized source rock intrusive mafic, volcanic mafic, basement mafic

\section{References}

Griscom, 1966 


\section{CAm31 scate !}

Province Coast Ranges

Tectonic Setting transcurrent, accretion

\section{Terrane Definition}

Narrow NW-trending magnetic high (100x10km).

\section{Hi/lo/both H}

\section{Geologic Province}

Located along fault traces of the San Andreas Fault zone, over K granitic rocks of the La Panza Range.

Inferred Source of Anomaly

Magnetic intrusive rocks.

Generalized source rock intrusive silicic

References 


\section{CAm32 sate}

Province Coast Ranges

Tectonic Setting transcurrent, accretion

\section{Terrane Definition}

Small oval magnetic high (20km).

Hi/lo/both $\mathrm{H}$

\section{Geologic Province}

Located over the eastern Coast Ranges on the San Andreas Fault SE of Parkfield over T sedimentary rocks, and near an outcrop of Cretaceous granite.

Inferred Source of Anomaly

Inferred to be due to buried body of magnetic serpentinite.

Generalized source rock ultramafic

References

Hanna and others, 1972 


\section{CAm33 \\ Scale}

Province Great Valley

Tectonic Setting accretion

Terrane Definition

Small oval magnetic high $(20 \mathrm{~km})$.

Hi/lo/both H

\section{Geologic Province}

Located over eastern part of Great Valley and within anomaly CAm26.

Inferred Source of Anomaly

May be a shallow sliver of obducted magnetic oceanic crust.

Generalized source rock ultramafic, intrusive mafic

References 


\section{CAm34 sate}

\section{Province Great Basin}

Tectonic Setting extension, batholith

\section{Terrane Definition}

NS-oriented magnetic high (100x50km).

\section{Hi/lo/both H}

\section{Geologic Province}

Located over Mz pluton associated with the southern Sierra Nevada batholith over the Argus Range.

\section{Inferred Source of Anomaly}

Magnetic intrusive rocks.

Generalized source rock intrusive mafic

References 


\section{CAm35 \\ Scale}

Province Mojave Desert

Tectonic Setting extension, batholith

Terrane Definition

Small magnetic high $(40 \times 40 \mathrm{~km})$.

Hi/lo/both $\mathrm{H}$

Geologic Province

Located mostly over $\mathrm{T}$ and $\mathrm{Q}$ volcanic and some Mz granitic rocks. Includes some outcrop of $\mathrm{pC}$ rocks.

Inferred Source of Anomaly

Magnetic volcanic or intrusive rocks

Generalized source rock volcanic mafic, intrusive mafic

References 
CAm36 salt

Province Great Basin

Tectonic Setting extension

Terrane Definition

Small oval magnetic high (10km).

Hi/lo/both $\mathrm{H}$

Geologic Province

Located over a $\mathrm{T}$ intrusive body.

Inferred Source of Anomaly

Magnetic intrusive rocks.

Generalized source rock intrusive mafic

References 


\section{CAm37 sate}

Province Great Valley

Tectonic Setting accretion

\section{Terrane Definition}

Small oval magnetic high $(20 \mathrm{~km})$.

Hi/lo/both $\mathrm{H}$

Geologic Province

Located over eastern part of Great Valley and within anomaly CAm26.

Inferred Source of Anomaly

May be a shallow sliver of obducted magnetic oceanic crust.

Generalized source rock ultramafic, intrusive mafic

References 


\section{CAm38 salb}

Province Great Basin, Mojave

Tectonic Setting transcurrent

\section{Terrane Definition}

ENE-trending linear feature $(\sim 300 \mathrm{~km})$ defined by the alignment of truncated prominent magnetic highs and lows of the Great Valley and Sierra Nevada (e.g. CAm26, CAm22, CAm17, CAm34). Also defined by left-lateral offset of prominant magnetic low (CAm22) and magnetic highs associated with Mz plutons (CAm17 and CAm39). Left-lateral offset of anomalies appears to be $\sim 75 \mathrm{~km}$. Approximately corresponds with trace of the Garlock Fault. Corresponds with gravity anomaly CAg35.

\section{Hi/lo/both B}

\section{Geologic Province}

Corresponds to left-lateral fault bounding southern edges of Coast Ranges, Great Valley, and Sierra Nevada with the Mojave desert.

\section{Inferred Source of Anomaly}

Edges of anomalies associated with Coast Ranges, Great Valley, Sierra Nevada, and Mojave Desert.

\section{Generalized source rock}




\section{CAm39 \\ Scale}

Province Mojave Desert

Tectonic Setting extension, batholith

\section{Terrane Definition}

Elongate WNW-trending magnetic high $(75 \times 25 \mathrm{~km})$.

Hi/lo/both $\mathrm{H}$

Geologic Province

Located mostly over Mz pluton in the Mojave Desert. SE end sits over volcanic rocks.

Inferred Source of Anomaly

Magnetic intrusive rocks.

Generalized source rock intrusive mafic

References 


\section{CAm40 sale}

Province Mojave Desert

Tectonic Setting extension, batholith

Terrane Definition

Small NW-trending magnetic high (30x10km).

Hi/lo/both $\mathrm{H}$

\section{Geologic Province}

Located over Mz pluton in Mojave Desert.

Inferred Source of Anomaly

Magnetic intrusive rocks.

Generalized source rock intrusive mafic

References 


\section{CAm41 seat}

Province Mojave Desert

Tectonic Setting batholith

Terrane Definition

Small NW-trending magnetic high (30x20km).

Hi/lo/both $\mathrm{H}$

\section{Geologic Province}

Located over $\mathrm{Mz}$ intrusive, Pz sedimentary and pC basement rocks in the Mojave Desert.

Inferred Source of Anomaly

Magnetic intrusive or basement rocks.

Generalized source rock intrusive mafic, basement mafic

References 


\section{IDg01}

Scale I

Province Snake River Plain

Tectonic Setting hotspot

Terrane Definition

Broad NW-trending high containing several smaller elongate anomalies (up to 200km). Gravity terrane is north of, and broader than, the magnetic terrane (IDm08). Contiguous with IDg06 and IDg04.

Hi/lo/both H

\section{Geologic Province}

Veneer of alluvium overlying basaltic lava flows.

Inferred Source of Anomaly

Dense and magnetic basaltic rocks.

Generalized source rock volcanic mafic

References

Mabey, 1976;

Mabey, 1982;

Sparlin and others, 1982;

Jachens and others, 1989 


\section{IDg02 \\ Scale $R$}

Province Northern Rocky Mtns

Tectonic Setting batholith

Terrane Definition

Gravity low bounded on west and south by western SRP high and on east by a gravity high (IDg03). Located over a regional magnetic low interspersed by isolated magnetic highs (e.g. IDm02).

Hi/lo/both L

Geologic Province

Granitic batholith.

Inferred Source of Anomaly

Low density, weakly magnetic granitic rocks.

Generalized source rock intrusive silicic

References

Bott and Smithson, 1967;

Jachens and others, 1989;

Mabey, 1986 


\section{IDg03}

Scale I

Province Northern Rocky Mtns

Tectonic Setting compression

Terrane Definition

Uniform gravity high bounded on south and east by intense gravity high of SRP and bounded on west by ID batholith. Regional magnetic low with local discrete highs.

Hi/lo/both $\mathrm{H}$

\section{Geologic Province}

Northernmost extension of basin and range. Mainly pC through upper Pz marine strata. Thick cover of Eocene volcanic rocks.

Inferred Source of Anomaly

Dense sedimentary and basement rocks.

Generalized source rock

basement silicic, basement carbonate, sedimentary carbonate, sedimentary siliceous

References 


\section{IDg04 \\ Scale I}

Province Snake River Plain

Tectonic Setting hotspot

Terrane Definition

Broad well defined high with several elongate large positive anomalies. Contiguous with IDg01 and IDg06. Very good correlation with magnetic anomaly IDm04.

Hi/lo/both H

\section{Geologic Province}

Basaltic lava flows. Located entirely within topographically defined SRP.

Inferred Source of Anomaly

Magnetic basaltic rocks.

Generalized source rock volcanic mafic

References

Mabey, 1976;

Sparlin and others, 1982;

Jachens and others, 1989 


\section{IDg05}

Scale

Province Snake River Plain

Tectonic Setting hotspot

Terrane Definition

NW-trending elongate gravity high $(50 \times 5 \mathrm{~km})$. Located mostly over a NW-trending elongate magnetic low.

Hi/lo/both H

\section{Geologic Province}

Located entirely within SRP over Q alluvium.

Inferred Source of Anomaly

Possibly dense, but weakly or reversely magnetized intrusive rocks.

Generalized source rock intrusive mafic, intrusive silicic

References 


\section{IDg06}

Scale $R$

Province Snake River Plain

Tectonic Setting hotspot

Terrane Definition

Arcuate regional gravity high, highest to the east. Contains several distinct smaller anomalies (e.g. IDg05,07,08). Contiguous with IDg01 and IDg04. Corresponds with magnetic high IDm10.

Hi/lo/both H

\section{Geologic Province}

Arcuate and well defined topographic depression. Mostly basaltic lava flows and cinder cones.

Inferred Source of Anomaly

Gravity highs due to dense mafic intrusive rocks and underplating.

Generalized source rock volcanic mafic, intrusive mafic

References

Mabey, 1976;

Mabey and others, 1978;

Mabey, 1982;

Sparlin and others, 1982;

Jachens and others, 1989 


\section{IDg07}

Scale I

Province Snake River Plain

Tectonic Setting hotspot

Terrane Definition

WNW-trending extremely high gravity terrane (150x25km). Contiguous with feature IDg08. Located entirely within larger western and eastern SRP gravity and magnetic terranes (IDg06,IDm08).

Hi/lo/both H

\section{Geologic Province}

Located entirely over $\mathrm{Q}$ and $\mathrm{T}$ volcanic rocks of the SRP.

Inferred Source of Anomaly

Likely source is dense basaltic lava flows and associated intrusive rocks.

Generalized source rock volcanic mafic, intrusive mafic

References 


\section{IDg08}

Scale I

Province Snake River Plain

Tectonic Setting hotspot

Terrane Definition

Irregular shaped, high gravity anomaly with many small frequency superimposed highs $(125 \mathrm{~km}$ EWx75km NS). Contiguous with gravity feature IDg07. Located entirely within larger western and eastern SRP gravity and magnetic terranes (IDg06,IDm08).

Hi/lo/both $\mathrm{H}$

Geologic Province

Located entirely over $\mathrm{Q}$ and $\mathrm{T}$ volcanic rocks of the SRP.

Inferred Source of Anomaly

Likely source is dense basaltic lava flows and associated intrusive rocks. High frequency superimposed anomalies may be due to volcanic vents.

Generalized source rock volcanic mafic, intrusive mafic

References 


\section{IDg09 \\ Scale I}

Province Snake River Plain

Tectonic Setting hotspot

Terrane Definition

NW-trending narrow gravity high $(50 \times 10 \mathrm{~km})$. Located at eastern edge of NW-trending magnetic feature IDm16.

Hi/lo/both H

\section{Geologic Province}

At edge of downwarp of SRP. Located over $\mathrm{Q}$ and $\mathrm{T}$ volcanic rocks at the southern edge of the SRP.

\section{Inferred Source of Anomaly}

May be volcanic infilling of dense, magnetic lava flows and possibly vents forming along tectonic edge of SRP.

Generalized source rock volcanic mafic, intrusive mafic

References 


\section{IDg10}

Province Snake River Plain

Tectonic Setting hotspot

Terrane Definition

EW-trending gravity high $(75 \times 25 \mathrm{~km})$. Corresponds with magnetic high IDm17.

Hi/lo/both H

Geologic Province

At edge of downwarp of SRP. Located over $\mathrm{Q}$ and $\mathrm{T}$ volcanic rocks at the southern edge of the SRP.

Inferred Source of Anomaly

May be volcanic infilling of dense, magnetic lava flows and possibly vents forming along tectonic edge of SRP.

Generalized source rock volcanic mafic, intrusive mafic

References

Mabey and others, 1978 


\section{IDm01}

Scale

Province Snake River Plain

Tectonic Setting hotspot

Terrane Definition

NW-trending magnetic high within SRP $(60 \times 25 \mathrm{~km})$. Located over broad gravity high.

Hi/lo/both H

Geologic Province

Located over SRP on T and Cretaceous sediments. Northeast edge borders on T volcanic rocks.

Inferred Source of Anomaly

Likely due to strongly magnetized intrusive or volcanic rocks.

Generalized source rock intrusive mafic, volcanic mafic

References 


\section{IDm02 \\ Scale I}

Province Northern Rocky Mtns

Tectonic Setting uplift

Terrane Definition

Irregular magnetic high (60x30km).

Hi/lo/both H

Geologic Province

Located over T intrusive on western edge of Idaho Batholith.

Inferred Source of Anomaly

Magnetic intrusive rocks.

Generalized source rock intrusive mafic

References

Mabey, 1986 


\section{IDm03}

\section{Scale}

Province Snake River Plain

Tectonic Setting hotspot

Terrane Definition

NW-trending prominent magnetic high within the SRP $(75 \times 25 \mathrm{~km})$.

Hi/lo/both H

\section{Geologic Province}

Located within SRP over Q sediments and some $\mathrm{Q}$ and $\mathrm{T}$ volcanic rocks. Anomaly is parallel to several NW-trending features crossing the eastern SRP.

Inferred Source of Anomaly

Magnetic intrusive or volcanic rocks.

Generalized source rock intrusive mafic, volcanic mafic

References 


\section{IDm04 \\ Scale I}

Province Snake River Plain

Tectonic Setting hotspot

Terrane Definition

Well defined region of small to intermediate sized $(50-75 \mathrm{~km})$ anomalies. Regionally a high magnetic area. Very good correlation with gravity anomaly IDg04. Contiguous with IDm08 and IDm10.

Hi/lo/both H

\section{Geologic Province}

Basaltic lava flows. Located entirely within topographically defined SRP.

Inferred Source of Anomaly

Dense basaltic rocks.

Generalized source rock volcanic mafic

References

Sparlin and others, 1982;

Mabey, 1986;

Jachens and others, 1989 


\section{IDm05}

\section{Scale}

Province Snake River Plain

Tectonic Setting hotspot

Terrane Definition

NW-trending elongate magnetic high within the western SRP (30x15km).

Hi/lo/both H

Geologic Province

Located within western SRP over Q volcanic rocks.

Inferred Source of Anomaly

Magnetic volcanic or buried intrusive rocks.

Generalized source rock intrusive mafic, volcanic mafic

References 


\section{IDm06}

Province Northern Rocky Mtns

Tectonic Setting compression

Terrane Definition

EW-trending string of local magnetic highs (75x25km).

Hi/lo/both H

Geologic Province

Located at south end of Idaho Batholith over Mz intrusive and T volcanic rocks.

Inferred Source of Anomaly

Magnetic volcanic or shallow intrusive rocks.

Generalized source rock intrusive mafic, volcanic mafic

References 


\section{IDm07

Province Northern Rocky Mtns, Snake River

Tectonic Setting hotspot, compression

\section{Terrane Definition}

NW-trending elongate string of magnetic highs $(125 \times 25 \mathrm{~km})$.

Hi/lo/both H

\section{Geologic Province}

Crosses at right angle to the northern edge of SRP and spans $\mathrm{Cz}$ volcanic, $\mathrm{Cz}$ and $\mathrm{Mz}$ intrusive, and Pz clastic rocks. Parallels trend of basins and ranges north of the SRP. Follows the NW-trending Craters of the Moon rift.

Inferred Source of Anomaly

Magnetic intrusive and volcanic rocks.

Generalized source rock intrusive mafic, volcanic mafic

References

Mabey and others, 1978 


\section{IDm08}

Province Snake River Plain

Tectonic Setting hotspot

Terrane Definition

NW-trending region consisting of several high frequency and some prominent long linear anomalies (up to $200 \mathrm{~km}$ ). Magnetic terrane is south of, and narrower than the gravity terrane (IDg1). Contiguous with IDm04 and IDm10.

Hi/lo/both H

\section{Geologic Province}

Veneer of alluvium overlying basaltic lava flows.

Inferred Source of Anomaly

Dense and magnetic basaltic rocks.

Generalized source rock volcanic mafic

References

Sparlin and others, 1982;

Mabey, 1982;

Jachens and others, 1989 


\section{IDm09}

Province Snake River Plain

Tectonic Setting hotspot

Terrane Definition

NW-trending prominent magnetic high within the eastern SRP (30x15km).

Hi/lo/both H

Geologic Province

Located within eastern SRP over Q volcanic rocks.

Inferred Source of Anomaly

Magnetic volcanic rocks or buried intrusive.

Generalized source rock volcanic mafic, intrusive mafic

References 


\section{IDm10 \\ Scale $R$}

Province Snake River Plain

Tectonic Setting hotspot

Terrane Definition

Arcuate regional magnetic high consisting of small to intermediate sized anomalies $(<100 \mathrm{~km})$ in most places displaying a NW-fabric (e.g. IDm03,07,09). Corresponds with gravity high IDg06. Contiguous with IDm04 and IDm08.

Hi/lo/both H

\section{Geologic Province}

Arcuate and well defined topographic depression. Mostly basaltic lava flows and cinder cones.

Inferred Source of Anomaly

Magnetic mafic volcanic and intrusive rocks.

Generalized source rock volcanic mafic, intrusive mafic

References

Mabey, 1976;

Mabey and others, 1978;

Mabey, 1982;

Sparlin and others, 1982;

Jachens and others, 1989 


\section{IDm11}

Scale

Province Great Basin, Middle Rocky Mtns

Tectonic Setting compression

\section{Terrane Definition}

Large, pronounced magnetic low $(250 \times 125 \mathrm{~km})$ that corresponds with gravity high extending off of the eastern SRP.

Hi/lo/both L

Geologic Province

Located over thrusted strata of the Rocky Mtns thrust belt.

Inferred Source of Anomaly

Weakly magnetic, high density carbonate rocks.

Generalized source rock sedimentary carbonate

References 


\section{IDm12}

Scale

Province Great Basin

Tectonic Setting batholith

Terrane Definition

Prominent magnetic low $(100 \times 50 \mathrm{~km})$ located over a moderate gravity low of the same general size.

Hi/lo/both L

Geologic Province

Located over Mz intrusive rocks. May be related to Idaho Batholith.

Inferred Source of Anomaly

Weakly magnetic and relatively low density intrusive rocks.

Generalized source rock intrusive silicic

References 


\section{IDm13}

\section{Scale}

Province Snake River Plain

Tectonic Setting hotspot

Terrane Definition

EW-trending prominent magnetic high within the eastern SRP (30x15km).

Hi/lo/both H

Geologic Province

Located within eastern SRP over Q volcanic rocks.

Inferred Source of Anomaly

Magnetic volcanic or buried intrusive rocks.

Generalized source rock volcanic mafic, intrusive mafic

References 


\section{IDm14 \\ Scale I}

Province Great Basin, Snake River Plain

Tectonic Setting hotspot

Terrane Definition

NW-trending subdued magnetic high (100x30km) branching off of eastern SRP. Bounds Magnetic low domain to northeast.

Hi/lo/both H

\section{Geologic Province}

Located over early Pz stratified rocks, $\mathrm{pC}$ metamorphic, and T sedimentary rocks. Parallels trend of thrust slices of Pz strata. Cuts across and is normal to the southern edge of SRP. Has similar trend to hotspot-related rift features such as the Great Rift and to narrow NW-trending features in Utah (UTg02, UTm02).

\section{Inferred Source of Anomaly}

May be similar in origin to feature UTm02 - rift related mafic magnetic intrusive or volcanic rocks or Precambrian basement.

Generalized source rock intrusive mafic,volcanic mafic, basement mafic

References

Mabey and others, 1978 


\section{IDm15 scate +}

Province Snake River Plain

Tectonic Setting hotspot

Terrane Definition

Irregular magnetic terrain similar in character with SRP. Forms a tongue extending off western SRP in line and parallel to eastern SRP. Includes a prominent U-shaped magnetic high at its SW end (IDm18). Possibly a fossil trace of Yellowstone hotspot. Forms part of the Humboldt magnetic zone of Mabey and others (1978).

Hi/lo/both $\mathrm{H}$

Geologic Province

Located over $\mathrm{T}$ and $\mathrm{Q}$ volcanic rocks.

Inferred Source of Anomaly

Magnetic volcanic and intrusive rocks.

Generalized source rock volcanic mafic, intrusive mafic

References

Mabey and others, 1978;

Hinze and Zietz, 1985 


\section{IDm16 Scate i}

Province Snake River Plain, Great Basin

Tectonic Setting hotspot

\section{Terrane Definition}

NW-trending narrow magnetic high $(190 \times 40 \mathrm{~km})$. Includes gravity IDg09, though largely located outside the gravity defined western SRP over regional gravity high of northern GB (NVg04). Northern end converges on the southern edge of the topographic and gravity defined SRP. Feature narrows to the NW.

Hi/lo/both H

\section{Geologic Province}

Northern 2/3 of anomaly parallels western SRP. Southern end extends over northern GB.

\section{Inferred Source of Anomaly}

Probably due to extensive magnetic volcanic and intrusive rocks.

Generalized source rock volcanic mafic, intrusive mafic

References

Mabey and others, 1978 


\section{IDm17 salb +}

Province Snake River Plain

Tectonic Setting hotspot

Terrane Definition

EW-trending magnetic high $(75 \times 10 \mathrm{~km})$. Corresponds with gravity high IDg10.

Hi/lo/both $\mathrm{H}$

\section{Geologic Province}

At edge of downwarp of SRP. Located over $\mathrm{Q}$ and $\mathrm{T}$ volcanic rocks at the southern edge of the SRP.

Inferred Source of Anomaly

May be volcanic infilling of dense, magnetic lava flows and possibly vents forming along tectonic edge of SRP.

Generalized source rock volcanic mafic, intrusive mafic

References

Mabey and others, 1978 


\section{IDm18}

\section{Scale}

Province Great Basin

Tectonic Setting hotspot, extension

Terrane Definition

U-shaped magnetic high $(75 \times 20 \mathrm{~km})$ that is located over a gravity high.

Hi/lo/both H

Geologic Province

Located over T lava flows.

Inferred Source of Anomaly

Magnetic volcanic and intrusive rocks.

Generalized source rock intrusive mafic, volcanic mafic

References 


\section{NVg01}

Province Great Basin

Tectonic Setting transcurrent, accretion

\section{Terrane Definition}

NE-trending boundary between high gravity to SE and low gravity to NW. Very well correlated with magnetic boundary. Corresponds with magnetic feature NVm05.

\section{Hi/lo/both B}

\section{Geologic Province}

West of this line there are no pre-Cz rocks exposed. The boundary also corresponds with the eastern edge of topographic and basaltic plateau. Note that this boundary corresponds with the edge of basement gravity calculation. See Isostatic gravity map.

Inferred Source of Anomaly

The prominent gravity anomaly likely reflects a major deep crustal discontinuity.

Generalized source rock volcanic mafic, sedimentary siliceous

References

McKee and others, 1983;

Blakely, 1988;

McKee and others, 1990;

Wyld and Wright, 2001 


\section{NVg02 \\ Scale I}

Province Great Basin

Tectonic Setting uplift

Terrane Definition

ENE-trending gravity high mainly defined in NW Nevada (225x100km).

Hi/lo/both H

\section{Geologic Province}

Located mostly over Mz metaclastic rocks (including mudstones and sandstones). Crosses several pre- $\mathrm{Cz}$ lithologic terranes and the continental margin inferred from Sr-isotopic ratios. Anomaly lies in line with eastern SRP and is coincident with a region of high heatflow extending SW from the eastern SRP. Anomaly is not easily distinguished from general gravity high south of the SRP. Northern edge of anomaly is poorly defined.

Inferred Source of Anomaly

May reflect a region of uplifted dense lower crustal rocks.

Generalized source rock basement mafic

References 


\section{NVg03 \\ Scale I}

Province Great Basin

Tectonic Setting extension

Terrane Definition

Oval gravity low (50km) within broad regional gravity high of northern GB (NVg04).

\section{Hi/lo/both L}

\section{Geologic Province}

Located over western assemblage (upper plate of Roberts Mt thrust) cherts and shales at north end of Carlin trend. Small plutons crop out at center of anomaly

Inferred Source of Anomaly

May be due to a large buried pluton.

Generalized source rock intrusive silicic

References 


\section{NVg04 \\ Scale $R$}

Province Great Basin

Tectonic Setting continental margin, hotspot

\section{Terrane Definition}

Regional gravity high across northern GB. Extends from the SRP southward. Southern boundary (at $40^{\circ}$ latitude) is marked by an abrupt gradient to low regional gravity of the southern GB regional gravity low (NVg10). Anomaly largely coincides with a regional magnetic low (UTm01).

Hi/lo/both H

\section{Geologic Province}

The central and eastern part of the anomaly is underlain largely by Pz carbonate rocks. The western and northern parts are coincident with $\mathrm{Pz}$ silicic sedimentary rocks overlain by Miocene volcanic rocks that extend north into OR, ID, and SRP.

\section{Inferred Source of Anomaly}

Underlain by low magnetic, high density (likely carbonate rocks). Anomaly may indicate that carbonate rocks extend to the SRP and that volcanic rocks form only a thin veneer (since magnetic anomaly is low). Alternatively, gravity high may reflect dense mafic underplating associated with the Yellowstone hotspot.

Generalized source rock sedimentary carbonate, intrusive mafic

References

Glen and others, 2000;

Pierce and others, 2000 


\section{NVg05}

Scale

Province Great Basin

Tectonic Setting subduction, continental margin

Terrane Definition

ENE-trending gravity high $(80 \times 25 \mathrm{~km})$. Corresponds with a magnetic low.

Hi/lo/both H

\section{Geologic Province}

Located over T volcanic, $\mathrm{Pz}$ and $\mathrm{Mz}$ sedimentary, and $\mathrm{Mz}$ intrusive rocks near the boundary of the Black Rock and Jungo lithologic terranes.

Inferred Source of Anomaly

May be due to dense intrusive or Mz metasedimentary rocks.

Generalized source rock basement mafic

References 


\section{NVg06}

Province Great Basin

Tectonic Setting continental margin

Terrane Definition

Regional gravity high. Generally associated with magnetic low, but also spans magnetic highs of western and central NNR.

Hi/lo/both H

\section{Geologic Province}

Located over T volcanic, Mz sedimentary and intrusive, and Pz sedimentary rocks.

Centered on Jurassic Humboldt lopolith gabbro and basalts.

\section{Inferred Source of Anomaly}

May be due to dense mafic intrusive rocks including the Jurassic Humboldt lopolith.

Generalized source rock intrusive mafic

References

Page, 1965;

Speed, 1976;

Oldow, 1984;

Dilek and Moores, 1995;

Hildenbrand and others, 2000 


\section{NVg07 \\ Scale I}

Province Great Basin

Tectonic Setting continental margin, accretion

\section{Terrane Definition}

Prominent gravity high over north central Nevada. Southern boundary of the high has a distinct ' $\mathrm{V}$ ' shape. Anomaly is part of the Northern GB gravity high terrane (NVg04). West edge of gravity ' $\mathrm{V}$ ' corresponds closely with the Northern Nevada Rift (NVm12).

\section{Hi/lo/both H}

\section{Geologic Province}

East edge of gravity 'V' corresponds with middle Pz Roberts Mt thrust front. West edge may reflect an older crustal discontinuity.

\section{Inferred Source of Anomaly}

Gravity high (inside 'V' relates to regional high of northern GB - NVg04). East edge may reflect crustal discontinuity at thrust juxtaposing dense $\mathrm{Pz}$ sedimentary rocks to the NW with less dense sedimentary rocks to the SE. West edge may reflect an older crustal discontinuity associated with the $\mathrm{pC}$ continental margin.

Generalized source rock sedimentary siliceous, basement mafic

References

Grauch and others, 1995;

Hildenbrand and others, 2000;

Ponce and Glen, 2002 


\section{NVg08}

\section{Scale}

Province Great Basin

Tectonic Setting subduction

Terrane Definition

Gravity high (125x60km). Corresponds with west end of feature NVm14 associated with diverse magnetic signature of the Walker Lane belt.

Hi/lo/both H

\section{Geologic Province}

Located largely over the Carson Sink and over T volcanic rocks on the west edge of anomaly. Also located over some $\mathrm{Mz}$ intrusive rocks.

\section{Inferred Source of Anomaly}

May be due to dense mafic to intermediate composition volcanic and associated intrusive rocks.

Generalized source rock volcanic mafic, intrusive mafic

References

Hildenbrand and others, 2000 


\section{NVg09 \\ Scale I}

Province Great Basin

Tectonic Setting subduction

Terrane Definition

NW-trending gravity low bounded to SW by the generally high gravity over the Walker Lane belt ( NVg11). Located over a regional magnetic low punctuated by highs of NNR anomalies. Bounded on the northeast by the gravity high of the Northern Nevada basement gravity V (NVg07). At its eastern edge it merges with feature NVg10.

\section{Hi/lo/both L}

\section{Geologic Province}

Located over intermediate composition tuffs that lie on Mz strata in the west, and on $\mathrm{Pz}$ strata in the east.

\section{Inferred Source of Anomaly}

Low density tuffs, and quartzose sedimentary rocks.

Generalized source rock sedimentary silicic

References 


\section{NVg10 \\ Scale $R$}

Province Great Basin

Tectonic Setting extension

Terrane Definition

Regional gravity low that extends from $\sim 37^{\circ}$ latitude in the south to $\sim 40^{\circ}$ latitude in the north. Anomaly located mostly over regional magnetic low (UTm01). On its western edge, anomaly merges with feature NVm09.

\section{Hi/lo/both L}

\section{Geologic Province}

Western part is located mostly over T volcanic and Pz sedimentary rocks. To the east, anomaly located mostly over Pz rocks. Pz rocks on the west are more silicic than those to the east. Region lacks significant basalt outcrops such as occur to the north.

\section{Inferred Source of Anomaly}

Anomaly due to low density and low magnetic source rocks, which may indicate that $\mathrm{Pz}$ rocks are thinner here than to the north beneath feature $\mathrm{NVg} 04$.

Generalized source rock sedimentary silicic

References

Blakely and Jachens, 1991;

Glen and others, 2000 


\section{NVg11}

Scale I

Province Great Basin

Tectonic Setting transcurrent, extension, continental margin

\section{Terrane Definition}

NW-trending elongate zone $(\sim 100 \mathrm{~km} \times 350 \mathrm{~km})$ in western NV, regional positive anomaly with several prominent local $(\sim 25 \mathrm{~km})$ positive anomalies. Located within diverse magnetic feature $\mathrm{NVm} 23$.

Hi/lo/both H

\section{Geologic Province}

Structurally defined province characterized by NW-trending topographic features. located over Mz granites, Pz strata, and late T volcanic rocks. Corresponds mostly with the physiographically defined Walker Lane belt.

\section{Inferred Source of Anomaly}

Gravity feature is possibly due to T mafic underplating. Magnetic anomalies may express shallow to deeply buried magnetic plutons. Possibly represents the eastern edge of Sierra Nevada Batholith.

Generalized source rock intrusive mafic

\section{References}

Blakely, 1988;

Craig, 1992;

Hildenbrand and others, 2000 


\section{NVg12}

\section{Scale I}

Province Great Basin

Tectonic Setting continental margin

\section{Terrane Definition}

Roughly oval (125km EWx100km NS) diffuse gravity high. Located over a regional magnetic low. Probably relates to feature NVg16.

Hi/lo/both H

Geologic Province

Located over diverse range of rock types including $\mathrm{T}$ volcanic and Pz sedimentary rocks.

Inferred Source of Anomaly

Likely source is thick accumulation of limestones and dolomites.

Generalized source rock sedimentary carbonate

References 


\section{NVg13}

Scale I

Province Great Basin

Tectonic Setting extension

Terrane Definition

Oval gravity low $(50 \mathrm{~km})$.

Hi/lo/both L

\section{Geologic Province}

Located over alluvial basin west of Wheeler Peak. NE end of anomaly located over granitic plutons.

Inferred Source of Anomaly

May be due to thick pile of low density alluvial fill, and/or low density granitic plutons.

Generalized source rock sedimentary siliceous, intrusive silicic

References 


\section{NVg14}

Scale I

Province Great Basin

Tectonic Setting batholith

Terrane Definition

Prominent NW-trending gravity low $(120 \times 30 \mathrm{~km})$ located over a magnetic low.

Hi/lo/both L

Geologic Province

Corresponds with the Jurassic Sylvania pluton in the Sylvania Mtns.

Inferred Source of Anomaly

Low density non-magnetic pluton.

Generalized source rock intrusive silicic

References

McKee, 1968 


\section{NVg15}

Province Great Basin

Tectonic Setting batholith

Terrane Definition

NS-trending gravity high $(60 \times 40 \mathrm{~km})$. Coresponds with intense parts of magnetic feature NV19.

Hi/lo/both H

\section{Geologic Province}

Located largely over T volcanic rocks. Some outcrops of Mz sedimentary and $\mathrm{T}$ intrusive rocks at the south end of anomaly.

Inferred Source of Anomaly

May be due to dense volcanic and associated intrusive rocks.

Generalized source rock volcanic mafic, intrusive mafic

References 


\section{NVg16}

Province Great Basin

Tectonic Setting continental margin

Terrane Definition

EW-trending variable gravity high $(200 \times 50 \mathrm{~km})$. Located over a regional magnetic low Probably relates to feature NVg12.

Hi/lo/both H

\section{Geologic Province}

Located over T volcanic and Pz carbonate rocks, though largely follows the along northern and western edge of an extensive outcrop of Pz carbonates.

Inferred Source of Anomaly

Dense, weakly magnetic carbonate rocks.

Generalized source rock sedimentary carbonate

References 
NVg17

Scale I

Province Great Basin

Tectonic Setting subduction

Terrane Definition

NW-trending gravity low (30x20km). Corresponds with magnetic anomaly NVm21.

\section{Hi/lo/both L}

\section{Geologic Province}

Wilson Creek Mtns - late Oligocene caldera.

\section{Inferred Source of Anomaly}

Thick accumulation of low density, magnetic caldera fill tuffs and underlying silicic intrusive rocks.

Generalized source rock volcanic silicic

References

Best and others, 1989a;

Best and others, 1989b 


\section{NVg18 \\ Scale I}

Province Great Basin

Tectonic Setting subduction, hotspot

\section{Terrane Definition}

Boundary between low basement gravity to the north and higher basement gravity to the south. Also an alignment of several prominent anomalies across Sierra Nevada, southern GB, and CP. No or little magnetic expression.

\section{Hi/lo/both B}

\section{Geologic Province}

Southern end of GB. Corresponds with topographic drop from north to south, and to a band of seismicity rimming the Basin and Range.

\section{Inferred Source of Anomaly}

Speculated (Saltus and Thompson, 1995) to relate to a combination of thermal buoyancy (related to Yellowstone plume head), thickened crust and mid-lower crustal felsic and mafic dikes and sill injection beneath the gravity high region.

Generalized source rock intrusive mafic, intrusive felsic

References

Stewart and others, 1977;

Eaton and others, 1978;

Saltus and Thompson 1995;

Pierce and others, 2000 


\section{NVg19}

Province Great Basin

Tectonic Setting extension

Terrane Definition

Irregular-shaped gravity low with local highs (175x100km). Corresponds to regional magnetic low.

Hi/lo/both L

Geologic Province

Located mostly over Pz carbonate rocks and Q and T sediments.

Inferred Source of Anomaly

May be due to thick low density, poorly consolodated sedimentary rocks in deep intra-range basins.

Generalized source rock sedimentary siliceous

References 


\section{NVg20 \\ Scale I}

Province Great Basin

Tectonic Setting subduction

Terrane Definition

Oval east-trending gravity low $(75 \times 40 \mathrm{~km})$. Partly coincident with anomaly NVm24.

Hi/lo/both L

Geologic Province

West end of Clover Mtns and part of Caliente depression and Caliente and Kane Springs Wash calderas. Also over Pz sedimentary rocks.

Inferred Source of Anomaly

Thick accumulation of low density rhyolitic and tuffaceous sedimentary rocks.

Generalized source rock volcanic silicic

References

Noble and McKee, 1972;

Best and others, 1989b 


\section{NVg21}

Scale I

Province Great Basin

Tectonic Setting extension, uplift

Terrane Definition

Irregular shaped gravity high. Generally NW-trending (max 300km NWx125km SE).

Hi/lo/both H

\section{Geologic Province}

Located over pC crystalline, Pz carbonate and quartzite, and T volcanic rocks.

Includes most of Death Valley and Pahrump Valley.

\section{Inferred Source of Anomaly}

Likely source is pC crystalline basement and thick accumulation of limestones and dolomites.

Generalized source rock basement silicic

References 


\section{NVg22 \\ Scale L}

Province Great Basin

Tectonic Setting uplift

Terrane Definition

Small oval gravity high $(10 \mathrm{~km})$. Corresponds partly with magnetic high NVm30.

Hi/lo/both H

Geologic Province

Fault block of pC to Mz sedimentary rocks.

Inferred Source of Anomaly

Speculated (Blank, 1988; Blakely, 1988) to be due to upwarp in dense and magnetic crystalline basement and to an associated $\mathrm{T}$ intrusion.

Generalized source rock basement mafic

References

Stewart and Carlson, 1978;

Blank, 1988;

Blakely, 1988 


\section{NVg23 \\ Scale I}

Province Great Basin

Tectonic Setting extension, uplift

Terrane Definition

Irregular shaped gravity high (140x125km). Bulk of anomaly located over magnetic low. Entire anomaly crosses numerous local magnetic anomalies.

Hi/lo/both $\mathrm{H}$

Geologic Province

Located largely over pC rocks of the Virgin Valley.

Inferred Source of Anomaly

Dense pC crystalline rocks.

Generalized source rock basement silicic

References

Langenheim and others, 2000 


\section{NVm01}

Scale

Province Great Basin

Tectonic Setting extension, hotspot

Terrane Definition

Elongate north-trending magnetic high (100x25km).

Hi/lo/both H

Geologic Province

Located over T volcanic and sedimentary rocks. Has similar trend as the western NNR.

Inferred Source of Anomaly

Strongly magnetic volcanic rocks or mafic intrusive rocks.

Generalized source rock intrusive mafic, volcanic mafic

References

Glen and Ponce, 2002;

Ponce and Glen, 2002 


\section{$\mathrm{NVm} 02$ \\ Scale L}

Province Great Basin

Tectonic Setting extension, hotspot

Terrane Definition

Oval cluster of high frequency positive magnetic anomalies $(25 \mathrm{~km})$.

Hi/lo/both H

Geologic Province

McDermitt group of caldera.

Inferred Source of Anomaly

Magnetic intrusive and extrusive rocks.

Generalized source rock intrusive mafic, volcanic silicic

References

U.S. Geological Survey 1972;

U.S. Geological Survey 1982a,b;

Rytuba and McKee, 1984;

Best and others, 1989b; 


\section{NVm03}

\section{Scale}

Province Great Basin

Tectonic Setting extension, hotspot

Terrane Definition

Elongate NW-trending moderate magnetic high (50x20km).

Hi/lo/both H

Geologic Province

Located over T volcanic rocks.

Inferred Source of Anomaly

Strongly magnetic volcanic rocks.

Generalized source rock volcanic mafic, intrusive mafic

References

Glen and Ponce, 2002;

Ponce and Glen, 2002 


\section{NVm04}

Province Great Basin

Tectonic Setting extension, hotspot

Terrane Definition

Subdued east-trending magnetic high (40x15km).

Hi/lo/both H

Geologic Province

At its west end it overlies a K pluton; middle and eastern portions located over $\mathrm{T}$ volcanic rocks.

Inferred Source of Anomaly

Moderately magnetic intrusive and/or volcanic rocks.

Generalized source rock volcanic mafic, intrusive silicic

References 


\section{NVm05 Scate I}

Province Great Basin

Tectonic Setting transcurrent, accretion

\section{Terrane Definition}

NE-trending boundary between strongly magnetic region of high frequency anomalies to the NW and low magnetic relief to the SE. Very good correlation with gravity boundary (NVg01).

\section{Hi/lo/both B}

\section{Geologic Province}

West of this line there are no pre-Cz rocks exposed. The boundary also corresponds with the eastern edge of topographic and basaltic plateau.

\section{Inferred Source of Anomaly}

Magnetic anomaly reflects contrast of magnetic volcanic rocks to NW and weakly magnetic sedimentary rocks to the SE.

Generalized source rock volcanic mafic, sedimentary siliceous

References

McKee and others, 1983;

Blakely, 1988;

McKee and others, 1990;

Wyld and Wright; 2001 


\section{NVm06}

\section{Scale}

Province Great Basin

Tectonic Setting extension, hotspot

Terrane Definition

Subdued north-trending magnetic high (50x15km).

Hi/lo/both H

\section{Geologic Province}

Located over T volcanic rocks and Mz sedimentary rocks. Has similar trend as the western NNR.

\section{Inferred Source of Anomaly}

May be due to magnetic mafic intrusive rocks.

Generalized source rock intrusive mafic, volcanic mafic

References

Glen and Ponce, 2002;

Ponce and Glen, 2002 


\section{NVm07

Province Great Basin

Tectonic Setting extension, hotspot

\section{Terrane Definition}

Oval $(20 \mathrm{~km})$ magnetic high located over the northern part of the western NNR (NVm08).

Hi/lo/both H

Geologic Province

Located just south of McDermitt Calderas, over T volcanic rocks.

Inferred Source of Anomaly

Perhaps due to magnetic intrusive rocks associated with the western NNR (NVm08).

Generalized source rock intrusive mafic, volcanic mafic

References

Glen and Ponce, 2002;

Ponce and Glen, 2002 


\section{NVm08 scal}

Province Great Basin

Tectonic Setting hotspot, extension

\section{Terrane Definition}

Two long, narrow NNW-trending magnetic highs that merge to the north (western and central Northern Nevada Rifts of Glen and Ponce, 2002).

\section{Hi/lo/both H}

\section{Geologic Province}

Anomaly corresponds in places with narrow NNW-trending zones of mafic dikes (of presumed Miocene age). Due to their great length, the anomalies likely correspond in places with other sources, such as the small magnetic high along central anomaly just south of $\mathrm{NVm} 07$. Inferred to have the same origin as anomaly NVm12.

\section{Inferred Source of Anomaly}

Sources are largely concealed. Largely due to magnetic mafic intrusive rocks.

Generalized source rock intrusive mafic

\section{References}

Blakely, 1988;

Glen and Ponce 2002;

Ponce and Glen, 2002 


\section{NVm09 Scale 1}

Province Great Basin

Tectonic Setting extension, depression

Terrane Definition

Magnetic low crossing eastern NNR (NVm12) magnetic high. Gap in eastern NNR corresponds with gravity low and topographic trough.

Hi/lo/both L

Geologic Province

NE-trending fault-bound basin (Midas Trough) cutting late $\mathrm{Cz}$ rocks.

Inferred Source of Anomaly

Downdropped basaltic rocks of eastern NNR (NVm12). Magnetic sources are farther from surface within trough than outside it.

Generalized source rock sedimentary siliceous

References

Rowan and Wetlaufer, 1973;

Zoback and Zoback, 1980;

Blakely, 1988 


\section{NVm10 \\ Scale}

Province Great Basin

Tectonic Setting extension

Terrane Definition

Oval magnetic high $(20 \mathrm{~km})$.

Hi/lo/both H

Geologic Province

Located mostly over Pz sedimentary rocks and some Tertiary volcanic and intrusive rocks. Lies over central Carlin trend.

Inferred Source of Anomaly

May be due to magnetic intrusive rocks.

Generalized source rock intrusive mafic

References

Henry and Ressel, 2000;

Grauch, 1996 


\section{NVm11 Scale ।}

Province Great Basin

Tectonic Setting extension, uplift

Terrane Definition

NE-trending elongate subdued magnetic high (100x25km).

Hi/lo/both $\mathrm{H}$

\section{Geologic Province}

Located over pC and Pz rocks of the Ruby Mtns metamorphic core complex (gneiss).

Inferred Source of Anomaly

Deep magnetic source of metamorphic rocks.

Generalized source rock metamorphic silicic, basement silicic

References

Dallmeyer and others, 1986;

Blakely, 1988;

Blakely and others, 1996 


\section{NVm12 \\ Scale I}

Province Great Basin

Tectonic Setting hotspot, extension

Terrane Definition

Long narrow NNW-trending magnetic high located over the eastern edge of a

prominent gravity gradient. Constitutes the eastern NNR of Glen and Ponce, 2002.

Hi/lo/both H

\section{Geologic Province}

Anomaly corresponds with narrow NNW-trending zone of Miocene mafic dikes.

Inferred Source of Anomaly

Magnetic mafic intrusive rocks.

Generalized source rock intrusive mafic

References

Zoback and Thompson, 1978;

Blakely 1988;

Zoback and others, 1994;

John and others, 2000;

Glen and Ponce 2002 


\section{NVm13}

Scale

Province Great Basin

Tectonic Setting extension

Terrane Definition

Elongate NW-trending moderate magnetic high (50x20km).

Hi/lo/both H

Geologic Province

Located over Pz carbonate rocks and several small $\mathrm{T}$ intrusive bodies.

Inferred Source of Anomaly

Magnetic intrusive rocks.

Generalized source rock intrusive mafic

References

Grauch, 1996 


\section{NVm14 \\ Scale I}

Province Great Basin

Tectonic Setting extension, transcurrent, batholith

Terrane Definition

Large varied NW-trending magnetic high $(250 \times 100 \mathrm{~km})$ consisting of many smaller isolated magnetic highs.

Hi/lo/both H

\section{Geologic Province}

Western half of anomaly lies over physiographically defined Walker Lane belt. Primarily large Mz plutons on the west and scattered plutons and Q and T volcanic rocks to east.

\section{Inferred Source of Anomaly}

Magnetic volcanic and intrusive rocks. Local highs may be volcanic centers or individual plutons.

Generalized source rock volcanic mafic, intrusive mafic

References

Blakely, 1988;

Stewart, 1988;

Hildenbrand and others, 2000 


\section{NVm15}

Province Great Basin

Tectonic Setting stable crust

Terrane Definition

Subdued NE-trending magnetic high (70x25km).

Hi/lo/both H

Geologic Province

Located mostly over Pz carbonate rocks.

Inferred Source of Anomaly

May be due to K intrusive rocks or mineralized carbonate strata.

Generalized source rock intrusive silicic

References

Mabey and others, 1978 


\section{NVm16 \\ Scale I}

Province Great Basin

Tectonic Setting extension, transcurrent, batholith

Terrane Definition

Irregular shaped magnetic high $(125 \times 50 \mathrm{~km})$. Contains a more prominent oval magnetic high at its southern edge.

Hi/lo/both H

\section{Geologic Province}

Located over $\mathrm{Q}$ and $\mathrm{T}$ volcanic rocks and Mz plutons. Centered over Aurora and Bodie igneous centers.

Inferred Source of Anomaly

Magnetic volcanic and intrusive rocks.

Generalized source rock intrusive mafic, volcanic mafic

References

Kleinhampl and others, 1975;

Grauch, 1996 


\section{NVm17

Province Great Basin

Tectonic Setting extension, transcurrent

Terrane Definition

"S"-shaped irregular magnetic high (40x10km).

Hi/lo/both $\mathrm{H}$

\section{Geologic Province}

Located over T volcanic rocks at south end of Toiyabe Range. North end corresponds to the Toiyabe caldera.

Inferred Source of Anomaly

May be due to magnetic volcanic and buried intrusive rocks.

Generalized source rock intrusive mafic, volcanic mafic References 


\section{NVm18 \\ Scale I}

Province Great Basin

Tectonic Setting extension, transcurrent,batholith

\section{Terrane Definition}

Small oval magnetic high (20km).

Hi/lo/both $\mathrm{H}$

Geologic Province

Located over Mt. Jefferson and Trail Canyon calderas in the Toquima Range

Inferred Source of Anomaly

Magnetic tuff and associated intrusive rocks.

Generalized source rock intrusive mafic, intrusive silicic

References

Kleinhampl and Ziony, 1984 


\section{NVm19 Scale I}

Province Great Basin

Tectonic Setting extension, subduction

\section{Terrane Definition}

Mostly east-trending, irregular shaped region of variable magnetic high (125x50km). Contains a more prominent oval magnetic high at its southern edge.

Hi/lo/both H

\section{Geologic Province}

Located mostly over T volcanic rocks of several identified volcanic centers. Few scattered $\mathrm{Mz}$ plutons and Pz sedimentary rocks.

Inferred Source of Anomaly

Magnetic volcanic and possibly intrusive rocks.

Generalized source rock volcanic mafic, volcanic silicic, intrusive silicic

References 


\section{NVm20 \\ Scale}

Province Great Basin

Tectonic Setting extension, subduction

Terrane Definition

Oval moderate magnetic high (30km).

Hi/lo/both H

Geologic Province

Located at east end of anomaly UTm15 and over T volcanic rocks.

Inferred Source of Anomaly

Magnetic volcanic rocks.

Generalized source rock volcanic mafic, volcanic silicic

References

Mabey and others, 1978 


\section{NVm21 \\ Scale I}

Province Great Basin

Tectonic Setting subduction

Terrane Definition

Magnetic high (25x15km) located within broader gravity low (NVg17).

Hi/lo/both H

Geologic Province

Wilson Creek Mtns - late Oligocene Indian Peak and White Rock calderas.

Inferred Source of Anomaly

Thick accumulation of low density, magnetic caldera fill tuffs and associated intrusive rocks.

Generalized source rock volcanic silicic

References

Best and others, 1989a;

Best and others, 1989b 


\section{NVm22 scale I}

Province Great Basin

Tectonic Setting batholith, transurrent

Terrane Definition

Subdued oval magnetic high mostly identified by a ring of maximum horizontal gradients $(20 \mathrm{~km})$.

Hi/lo/both H

\section{Geologic Province}

Located over pC and Cambrian sedimentary rocks and small exposure of $\mathrm{T}$ volcanic rocks.

\section{Inferred Source of Anomaly}

Oval form suggests it may be a buried pluton.

Generalized source rock intrusive silicic

References 


\section{NVm23 scale I}

Province Great Basin

Tectonic Setting transcurrent, extension, continental margin

\section{Terrane Definition}

NW-trending elongate region with high frequency NW elongate positive anomalies. Region is $\sim 250 \times 550 \mathrm{~km}$ in western and central NV. Includes several large anomalies (e.g. NVm14,16,17,19,22,25). Southwest part of anomaly located over gravity anomaly NVg11.

\section{Hi/lo/both H}

\section{Geologic Province}

Physiographically defined province characterized by NW-trending topographic features. located over $\mathrm{Mz}$ granites, $\mathrm{Pz}$ strata, and late $\mathrm{T}$ volcanic rocks. Western part of anomaly corresponds with the tectonically defined Walker Lane.

\section{Inferred Source of Anomaly}

Gravity terrane is possibly due to mafic underplating. Magnetic anomalies may express shallow to deeply buried magnetic plutons. Possible represents the eastern edge of Sierra Nevada Batholith.

\section{Generalized source rock intrusive mafic}

\section{References}

Blakely, 1988;

Craig, 1992;

Hildenbrand and others, 2000;

Blakely and others, 1996 


\section{NVm24 scate I}

Province Great Basin

Tectonic Setting subduction

Terrane Definition

A region of variable magnetic highs. EW-oriented oval feature $(75 \mathrm{~km} \times 50 \mathrm{~km})$ slightly displaced to north of a similarly shaped gravity low (NVg20).

Hi/lo/both $\mathrm{H}$

Geologic Province

Clover Mtns region - Miocene Caliente caldera.

Inferred Source of Anomaly

Magnetic volcanic tuffs and associated intrusive rocks.

Generalized source rock volcanic silicic, intrusive silicic

References

Best and others, 1989a;

Best and others, 1989b 


\section{NVm25 Scate I}

Province Great Basin

Tectonic Setting subduction, transcurrent

\section{Terrane Definition}

NW-trending magnetic high $(90 \times 25 \mathrm{~km})$ located over a basement gravity low. Corresponds partly with the single largest isostatic gravity low in Nevada (Silent Canyon Caldera).

Hi/lo/both H

\section{Geologic Province}

Located over the southern Nevada volcanic field - Miocene silicic volcanic rocks. Several identified calderas (e.g. Silent Canyon, Timber Mtn, Black Mtn).

Inferred Source of Anomaly

Magnetic, low density volcanic rocks.

Generalized source rock volcanic silicic

References

Eckel, 1968;

Byers and others, 1976;

Oliver and others, 1995;

Grauch and others, 1999;

Mankinen and others, 1999;

McKee and others, 1999;

Hildenbrand and others, 2000; 


\section{NVm26 scale I}

Province Great Basin

Tectonic Setting uplift

Terrane Definition

Small pronounced oval magnetic high $(20 \mathrm{~km})$. Located over a much broader pronounced gravity high (NVg23).

Hi/lo/both H

\section{Geologic Province}

Mormon Mtns--Fault block of pC crystalline basement.

Inferred Source of Anomaly

Dense and magnetic crystalline basement.

Generalized source rock basement mafic

References

Blank, 1988;

Blakely, 1988;

Blakely and others, 1996 


\section{NVm27 \\ Scale I}

Province Great Basin

Tectonic Setting uplift

Terrane Definition

Small subdued oval magnetic high $(40 \mathrm{~km})$. Located over a regional gravity high.

Hi/lo/both H

\section{Geologic Province}

Fault block of pC crystalline basement.

Inferred Source of Anomaly

Dense and magnetic crystalline basement.

Generalized source rock basement mafic

References

Blank, 1988;

Blakely, 1988;

Blakely and others, 1996 


\section{NVm28 scate +}

Province Great Basin

Tectonic Setting uplift

Terrane Definition

Small pronounced oval magnetic high $(20 \mathrm{~km})$. Located over a much broader pronounced gravity high (NVg23).

Hi/lo/both $\mathrm{H}$

Geologic Province

Fault block of pC crystalline basement.

Inferred Source of Anomaly

Dense and magnetic crystalline basement.

Generalized source rock basement mafic

References 


\section{NVm29 Scate I}

Province Great Basin

Tectonic Setting extension, uplift

Terrane Definition

EW alignment of magnetic highs that separates a regional magnetic low to north from a region dominated by several local magnetic highs to south.

Hi/lo/both H

\section{Geologic Province}

South of this boundary are $\mathrm{Q}$ volcanic, $\mathrm{T}$ intrusive, and $\mathrm{Mz}$ granititic rocks. To the north are mainly $\mathrm{Pz}$ carbonate rocks.

Inferred Source of Anomaly

Weakly magnetic carbonate rocks to north and magnetic igneous rocks to south.

Generalized source rock sedimentary carbonate, volcanic mafic, basement mafic

References

Blakely and others, 1996 


\section{NVm30 \\ Scale L}

Province Great Basin

Tectonic Setting uplift

Terrane Definition

Small oval magnetic high $(30 \mathrm{~km})$ enclosing a smaller NS elongate high. Located over NW flank of a broad NW-trending magnetic high $(60 \times 30 \mathrm{~km})$. Corresponds partly with gravity high NVg22.

Hi/lo/both H

Geologic Province

Fault block of pC to Mz sedimentary rocks.

Inferred Source of Anomaly

Possibly due to upwarp in dense and magnetic crystalline basement and to an associated $\mathrm{T}$ intrusion.

Generalized source rock basement mafic

References

Stewart and Carlson, 1978;

Blank, 1988;

Blakely, 1988;

Blakely and others, 1996 


\section{ORg01 \\ Scale I}

Province Cascade Mtns

Tectonic Setting accretion, subduction

Terrane Definition

Subdued NS-elongate gravity high $(>125 x>75 \mathrm{~km})$. Corresponds with magnetic anomaly ORm01.

Hi/lo/both H

\section{Geologic Province}

Located over western Cascades, late $\mathrm{Cz}$ volcanic rocks. Eastern edge is at the transition between the basaltic western Cascades and the younger, more andesitic eastern Cascades.

\section{Inferred Source of Anomaly}

Interpreted to be due to concealed accreted Eocene Siletz River volcanic rocks.

Forms part of the eastern edge of the Oregon forearc block.

Generalized source rock volcanic mafic

References

Wells and others, 1998 


\section{ORg02 \\ Scale I}

Province Columbia Plateaus

Tectonic Setting hotspot

Terrane Definition

Prominent oval gravity high $(50 \mathrm{~km})$ located at SW end of feature ORg03. Corresponds partly with magnetic anomaly ORm07.

Hi/lo/both H

Geologic Province

Located over T volcanic rocks.

Inferred Source of Anomaly

Perhaps due to dense magnetic intrusive rocks.

Generalized source rock intrusive mafic, volcanic mafic

References 


\section{ORg03 \\ Scale I}

Province Columbia Plateaus

Tectonic Setting hotspot, accretion

Terrane Definition

NE-trending elongate gravity high $(>125 \times 40 \mathrm{~km})$. Corresponds with magnetic anomaly ORm03.

Hi/lo/both H

\section{Geologic Province}

Skirts north edge of Blue Mtns and located mostly within the southern part of the John Day basin.

Inferred Source of Anomaly

Perhaps due to dense magnetic rift intrusive rocks or accreted Triassic ultramafic rocks.

Generalized source rock intrusive mafic, volcanic mafic, ultramafic

References 


\section{ORg04 \\ Scale $R$}

Province Cascade Mtns

Tectonic Setting subduction

Terrane Definition

NNW-elongate gravity low (50x175km). Closely correlated with magnetic high ORm08.

Hi/lo/both L

Geologic Province

Western Cascade Range consisting of $\mathrm{T}$ andesite volcanic rocks in the Cascade graben.

Inferred Source of Anomaly

Magnetic, low density intermediate volcanic rocks.

Generalized source rock volcanic silicic, intrusive silicic

References

Jachens and others, 1989;

LaFehr, 1965;

Blakely and others, 1985 


\section{ORg05}

\section{Scale}

Province Cascade Mtns, Columbia Plateaus

Tectonic Setting subduction

\section{Terrane Definition}

NNW-trending gravity low $(100 \times 25 \mathrm{~km})$. Contains discrete lows within it.

\section{Hi/lo/both L}

Geologic Province

Located over $\mathrm{Q}$ and $\mathrm{T}$ volcanic rocks of the high Cascade Range. Includes Crater Lake.

Inferred Source of Anomaly

Magnetic, low density intermediate volcanic rocks.

Generalized source rock volcanic silicic intrusive silicic

References 


\section{ORg06 \\ Scale $R$}

Province Columbia Plateaus

Tectonic Setting extension

Terrane Definition

Moderate gravity low with a few small $(<100 \mathrm{~km}$ diameter $)$ intermediate amplitude highs. Regional low is mainly defined by highs to east associated with northern GB and western SRP. Corresponds in part with magnetic anomaly ORm06.

Hi/lo/both B

\section{Geologic Province}

Southernmost edge of CRP basalts and related lava flows, and younger lava flows across Oregon highlands. lava flows are underlain by Tertiary sediments.

Inferred Source of Anomaly

Low density sedimentary rocks.

Generalized source rock sedimentary siliceous

References 


\section{ORm01 scalo -}

Province Cascade Mtns

Tectonic Setting accretion, subduction

\section{Terrane Definition}

Pronounced magnetic high $(>75 \mathrm{~km}$ NS $x>60 \mathrm{~km} \mathrm{EW).} \mathrm{Occupies} \mathrm{northern} 2 / 3$ of the corresponding gravity anomaly. Corresponds with gravity anomaly ORg01.

\section{Hi/lo/both H}

\section{Geologic Province}

Located over western Cascades, late $\mathrm{Cz}$ volcanic rocks. Eastern edge is at the transition between the basaltic western Cascades and the younger, more andesitic eastern Cascades.

\section{Inferred Source of Anomaly}

Interpreted to be due to concealed accreted Eocene Siletz River Volcanic rocks. Forms part of the eastern edge of the Oregon forearc block.

Generalized source rock volcanic mafic

References

Wells and others, 1998 


\section{ORm02 sale}

Province Cascade Mtns, Columbia Plateaus

Tectonic Setting subduction

Terrane Definition

Elongate NS-oriented magnetic high (50x20km).

Hi/lo/both $\mathrm{H}$

Geologic Province

Located over $\mathrm{T}$ and $\mathrm{Q}$ volcanic rocks of the Cascade Range that includes the Three

Sisters and Mt. Washington.

Inferred Source of Anomaly

Strongly magnetic volcanic rocks.

Generalized source rock volcanic silicic intrusive silicic

References 


\section{ORm03 scate +}

Province Columbia Plateaus

Tectonic Setting hotspot, accretion

\section{Terrane Definition}

NE-trending elongate magnetic high $(>125 \times 25 \mathrm{~km})$. Corresponds with gravity anomaly ORg03.

\section{Hi/lo/both H}

\section{Geologic Province}

Skirts north edge of Blue Mtns and located mostly within the southern part of the John Day basin.

\section{Inferred Source of Anomaly}

Perhaps due to dense magnetic rift intrusive rocks or accreted Triassic ultramafic rocks.

Generalized source rock intrusive mafic, volcanic mafic, ultramafic

$$
\text { References }
$$




\section{ORm04

Province Columbia Plateaus, Great Basin

Tectonic Setting accretion

Terrane Definition

Large NE-trending magnetic low (225x60km).

Hi/lo/both L

Geologic Province

Corresponds closely with Mz sedimentary rocks of the Blue Mtns.

Inferred Source of Anomaly

Weakly magnetic sedimentary rocks. Mainly contrasted with the surrounding strongly magnetic volcanic rocks.

Generalized source rock sedimentary siliceous

References

Vallier and Brooks, 1986;

Vallier and Brooks, 1994 


\section{ORm05}

\section{Scale}

Province Columbia Plateaus

Tectonic Setting subduction

Terrane Definition

Elongate arcuate NS-trending magnetic high (100x25km).

Hi/lo/both H

Geologic Province

Located over $\mathrm{Q}$ and $\mathrm{T}$ volcanic rocks on the east side of the Cascade Range (includes Newberry Craters).

Inferred Source of Anomaly

Strongly magnetic volcanic rocks.

Generalized source rock volcanic mafic, intrusive mafic

References 


\section{ORm06 Scale R}

Province Columbia Plateaus

Tectonic Setting extension, hotspot

\section{Terrane Definition}

High amplitude, high frequency magnetic anomalies that extend across Oregon highlands. Corresponds with gravity anomaly ORg06.

\section{Hi/lo/both B}

\section{Geologic Province}

Southernmost edge of CRP basalts and related rocks, and younger volcanic rocks across Oregon highlands. Volcanic rocks are underlain by Tertiary sediments.

Inferred Source of Anomaly

Magnetic basaltic rocks.

Generalized source rock volcanic mafic

References 


\section{ORm07 scate +}

Province Columbia Plateaus

Tectonic Setting hotspot

\section{Terrane Definition}

Oval magnetic high $(40 \mathrm{~km})$ that is located at the SW end of feature ORm03. Corresponds partly with gravity anomaly ORg02.

Hi/lo/both $\mathrm{H}$

\section{Geologic Province}

Located over T volcanic rocks.

Inferred Source of Anomaly

Perhaps due to dense magnetic intrusive rocks.

Generalized source rock intrusive mafic, volcanic mafic References 


\section{ORm08 scate $\mathrm{R}$}

Province Cascade Mtns

Tectonic Setting subduction

\section{Terrane Definition}

NNW-elongate magnetic high (50x200km). Closely correlated with gravity high ORg04. Consists of high amplitude and high frequency magnetic anomalies.

Hi/lo/both $\mathrm{H}$

Geologic Province

Western Cascade Range consisting of T andesite in the Cascade graben.

Inferred Source of Anomaly

Magnetic, low density intermediate volcanic rocks.

Generalized source rock volcanic silicic, intrusive silicic

References

LaFehr, 1965;

Blakely and others, 1985;

Jachens and others, 1989 


\section{ORm09 sate}

Province Columbia Plateaus

Tectonic Setting extension, hotspot

Terrane Definition

Elongate NW-trending magnetic high (60x10km).

Hi/lo/both $\mathrm{H}$

\section{Geologic Province}

Located over $\mathrm{Q}$ and $\mathrm{T}$ volcanic and sedimentary rocks. Anomaly has the same trend as $\mathrm{Q}$ faulting.

Inferred Source of Anomaly

Strongly magnetic volcanic rocks.

Generalized source rock volcanic mafic, intrusive mafic

References 


\section{ORm10 sate}

Province Columbia Plateaus

Tectonic Setting extension, hotspot

Terrane Definition

Elongate NW-trending magnetic high (50x20km).

Hi/lo/both $\mathrm{H}$

\section{Geologic Province}

Located over T volcanic and sedimentary rocks. Anomaly has the same trend as $\mathrm{Q}$ faulting.

Inferred Source of Anomaly

Strongly magnetic volcanic rocks.

Generalized source rock volcanic mafic, intrusive mafic

References 


\section{ORm11 seat}

\section{Province Great Basin}

Tectonic Setting extension, hotspot

\section{Terrane Definition}

Elongate north-trending magnetic high (80x20km).

\section{Hi/lo/both H}

\section{Geologic Province}

Located over Q and T volcanic rocks.

Inferred Source of Anomaly

Strongly magnetic volcanic rocks.

Generalized source rock volcanic mafic, intrusive mafic

$$
\text { References }
$$




\section{ORm12 seat}

\section{Province Great Basin}

Tectonic Setting hotspot

\section{Terrane Definition}

Elongate NE-trending magnetic high (125x25km).

\section{Hi/lo/both H}

\section{Geologic Province}

Located over $\mathrm{Q}$ and $\mathrm{T}$ volcanic and sedimentary rocks.

Inferred Source of Anomaly

Strongly magnetic volcanic rocks.

Generalized source rock volcanic mafic, intrusive mafic

References 


\section{ORm13 seat}

\section{Province Great Basin}

Tectonic Setting extension, hotspot

\section{Terrane Definition}

Elongate east-trending prominent magnetic high (75x40km).

\section{Hi/lo/both H}

\section{Geologic Province}

Located over T volcanic rocks of the Cascade Range.

Inferred Source of Anomaly

Strongly magnetic volcanic rocks.

Generalized source rock volcanic mafic, intrusive mafic

$$
\text { References }
$$




\section{ORm14 \\ Scale}

Province Great Basin

Tectonic Setting hotspot

Terrane Definition

Elongate NS-trending magnetic high (150x20km).

Hi/lo/both H

\section{Geologic Province}

Located over T volcanic rocks of Steens Mt. Anomaly has similar trend as the western NNR anomaly (feature NVm08).

Inferred Source of Anomaly

Strongly magnetic volcanic or mafic intrusive rocks.

Generalized source rock volcanic mafic, intrusive mafic

References 


\section{ORm15 \\ Scale}

\section{Province Great Basin}

Tectonic Setting hotspot

\section{Terrane Definition}

Elongate NS-trending magnetic high (60x20km).

Hi/lo/both H

\section{Geologic Province}

Located over $\mathrm{Q}$ and T volcanic rocks. Has similar trend and is in-line with the the western NNR anomaly (NVm08).

\section{Inferred Source of Anomaly}

Strongly mafic magnetic volcanic or intrusive rocks.

Generalized source rock volcanic mafic, intrusive mafic

References 


\section{UTg01}

\section{Scale I}

Province Great Basin

Tectonic Setting uplift, extension

Terrane Definition

Prominent NS-oriented oblate gravity high $(75 \times 30 \mathrm{~km})$. Located over regional magnetic low.

Hi/lo/both H

\section{Geologic Province}

Located over Great Salt Lake desert. Few outcrops nearby are T silicic volcanic rocks and Pz quartzites and limestones.

Inferred Source of Anomaly

Likely source is pC crystalline basement below Pz stratified rocks.

Generalized source rock basement mafic

References 


\section{UTg02 \\ Scale I}

Province Great Basin

Tectonic Setting hotspot

Terrane Definition

Prominent NW-trending gravity highs (UTg02a,b; 225x50km) branching off of the Eastern SRP. Anomaly has two segments. Corresponds with magnetic anomaly UTm02.

\section{Hi/lo/both H}

\section{Geologic Province}

Located over the Great Salt Lake. Anomaly merges with the Great Rift that cuts across the eastern SRP. Has same trend as: $\mathrm{Pz}$ and $\mathrm{pC}$ basement outcrops, the Great Salt Lake, and the Great Rift. Located over pC basement in the Raft River and Albion Mtns at its NW end.

\section{Inferred Source of Anomaly}

Possibly due to dense, magnetic mafic rocks associated with rifting. Has similar trend to basement on either side of eastern SRP. May be due to magnetic basement or to rift related intrusive rocks that were injected along pre-existing basement structure.

Generalized source rock intrusive mafic

References

Mabey and others, 1978;

Kuntz and others, 1988;

Kuntz and others, 1992 


\section{UTg03 \\ Scale I}

Province Great Basin

Tectonic Setting depression

Terrane Definition

Prominent gravity low $(130 \times 100 \mathrm{~km})$.

\section{Hi/lo/both L}

\section{Geologic Province}

Anomaly located mostly over $\mathrm{T}$ and $\mathrm{Q}$ alluvium. Eastern edge corresponds to the Rocky Mtns frontal thrust belt.

\section{Inferred Source of Anomaly}

Low density sedimentary basin fill. Likely due to thrust-related thickening of low density sedimentary rocks.

Generalized source rock sedimentary siliceous

References 


\section{UTg04 \\ Scale I}

Province Great Basin

Tectonic Setting subduction

Terrane Definition

EW-trending gravity low west of boundary between Uinta Basin and Mtns (75x30km). Marks the southern edge of the Great Salt Lake Desert. Corresponds with magnetic anomaly UTm05.

\section{Hi/lo/both L}

\section{Geologic Province}

Corresponds with T intrusive rocks and stratified Pz sandstones. May be due to subduction-related volcanism along pC crustal discontinuity.

Inferred Source of Anomaly

Low density intrusive rocks.

Generalized source rock intrusive silicic, sedimentary siliceous

References

Mabey and others, 1978 


\section{UTg05}

\section{Scale I}

Province Great Basin, Middle Rocky Mtns

Tectonic Setting accretion

\section{Terrane Definition}

EW-trending alignment of anomalies, comprising Uinta Mtns/Basin boundary to the east and a gravity trough (UTg04) to the west.

Hi/lo/both B

\section{Geologic Province}

Correlates with inferred boundary between Proterozoic crust to the south and Archean crust to the north.

\section{Inferred Source of Anomaly}

Defined by juxtaposing terranes on Proterozoic crust to the south (e.g. Uinta Mtns) and Archean crust to the north.

Generalized source rock basement mafic, basement silicic

References

Zietz and others, 1969;

Stewart and others, 1977;

Hutchinson and Albers, 1992;

Karlstrom and others, 2002 


\section{UTg06}

Scale I

Province Middle Rocky Mtns, Great Basin

Tectonic Setting uplift

Terrane Definition

Prominent east-trending gravity high (200x50km) over the Uinta Mtns. Located over a regional magnetic low. An oval (25km diameter) magnetic high (feature UTm03) is located at the east end of the Uinta Mtns.

\section{Hi/lo/both B}

\section{Geologic Province}

Closely corresponds to pC outcrops of the Uinta Mtns.

Inferred Source of Anomaly

Dense, generally weakly magnetic structurally uplifted $\mathrm{pC}$ basement.

Generalized source rock basement mafic

References

Simpson and others, 1986 


\section{UTg07}

Scale I

Province Middle Rocky Mtns, Great Basin,

Tectonic Setting depression

Terrane Definition

Prominent EW-elongate gravity low (200x75km). Corresponds partly with magnetic anomaly UTm06.

Hi/lo/both L

Geologic Province

Corresponds with the Uinta Basin.

Inferred Source of Anomaly

Due to low density sedimentary fill.

Generalized source rock sedimentary siliceous, volcanic mafic, intrusive mafic References 


\section{UTg08}

Scale I

Province Colorado Plateaus

Tectonic Setting uplift

Terrane Definition

Narrow EW-elongate gravity high (100x25km). Located over regional magnetic high.

Hi/lo/both $\mathrm{H}$

Geologic Province

Located at southern edge of the Uinta Basin.

Inferred Source of Anomaly

Source may be pC basement similar to Uinta Mtns.

Generalized source rock basement mafic

References 


\section{UTg09 \\ Scale I}

Province Colorado Plateaus

Tectonic Setting depression

Terrane Definition

EW-elongate gravity low $(125 \times 25 \mathrm{~km})$. Corresponds with magnetic anomaly UTm09.

\section{Hi/lo/both L}

\section{Geologic Province}

Located at southern edge of Uinta Basin. Anomaly parallels trend of Uinta Mtns.

Inferred Source of Anomaly

Likely reflects low density basin fill.

Generalized source rock sedimentary siliceous

References

Mabey and others, 1978 


\section{UTg10 \\ Scale I}

Province Great Basin

Tectonic Setting continental margin, uplift

\section{Terrane Definition}

Prominent gravity high (150x100km). Corresponds with a regional magnetic low.

\section{Hi/lo/both H}

\section{Geologic Province}

Located largely over $\mathrm{Q}$ deposits. Pz carbonate rocks correspond closely with anomaly's southern and eastern edges. Located also over scattered outcrops of $\mathrm{T}$ volcanic, Pz carbonate, and $\mathrm{pC}$ basement rocks.

\section{Inferred Source of Anomaly}

Perhaps due to thick accumulation of Pz carbonate rocks and/or to dense $\mathrm{pC}$ crystalline basement.

Generalized source rock sedimentary carbonate, basement mafic

References 


\section{UTg11}

Province Colorado Plateaus, Great Basin

Tectonic Setting uplift

Terrane Definition

NE-trending gravity high $(150 \times 50 \mathrm{~km})$ that encompasses the Wasatch Plateau. Northern end located over a magnetic low and southern end over a magnetic high (UTm11).

Hi/lo/both H

\section{Geologic Province}

Located at north end of basin along the Sevier River over western Colorado

Plateau. Corresponds with the western part of the San Rafel Swell.

Inferred Source of Anomaly

Likely reflects upwarp of dense $\mathrm{pC}$ basement.

Generalized source rock basement mafic

References 


\section{UTg12 \\ Scale I}

Province Colorado Plateaus

Tectonic Setting stable crust

Terrane Definition

NW-trending gravity low $(125 \times 50 \mathrm{~km})$ with distinct $\mathrm{NW}$-trending ridges. Located over a generally low magnetic area.

Hi/lo/both L

Geologic Province

Located over La Sal Mtns. Straddles Northern edge of CRP.

Inferred Source of Anomaly

Low may be partly due to La Sal T intrusive bodies. Ridges may be related to faults in $\mathrm{pC}$ basement.

Generalized source rock intrusive silicic

References 


\section{UTg13}

Scale I

Province Colorado Plateaus

Tectonic Setting uplift

Terrane Definition

NE-trending gravity high (170x50km). Corresponds with magnetic anomaly UTm14.

\section{Hi/lo/both H}

\section{Geologic Province}

Roughly corresponds to the edge of the Wasatch plateau. Cuts across drainages of the CP. Located over part of the Circle Cliffs and San Rafael Swell structural uplifts.

Inferred Source of Anomaly

Likely reflects upwarp of dense, magnetic pC basement.

Generalized source rock basement mafic

References 
UTg14

Scale I

Province Great Basin

Tectonic Setting uplift

Terrane Definition

NS-oriented gravity high $(100 \times 40 \mathrm{~km})$ that is contiguous with feature UTg10. North end crosses large magnetic high (UTm15). South end located over magnetic low region.

Hi/lo/both H

\section{Geologic Province}

Located at edge of GB over the eastern edge of the Sevier Thrust belt. Located mostly over T volcanic rocks.

Inferred Source of Anomaly

Unknown source, but possibly dense basement brought near to surface by frontal thrust.

Generalized source rock basement mafic

References 


\section{UTg15}

Scale I

Province Great Basin

Tectonic Setting subduction

Terrane Definition

Oval gravity low $(50 \mathrm{~km})$. Located over a magnetic low.

Hi/lo/both L

\section{Geologic Province}

Located over welded silicic tuff volcanic field (Bull Valley silicic complex).

Inferred Source of Anomaly

Thick accumulation of low density rhyolitic and tufaceous sedimentary rocks.

Generalized source rock volcanic silicic, intrusive silicic

References 


\section{UTg16}

Scale I

Province Colorado Plateaus

Tectonic Setting depression

Terrane Definition

Large oval gravity low $(100 \mathrm{~km})$. Located over moderately high magnetic terrain that includes magnetic anomalies AZm02 and southern end of UTm14.

\section{Hi/lo/both L}

\section{Geologic Province}

Located over Mz and Pz strata of CRP over Glen Canyon along the Colorado River.

\section{Inferred Source of Anomaly}

Area is covered by CRP strata. Possibly due to downwarp of basement.

Generalized source rock basement silicic

References 


\section{UTg17 \\ Scale I}

Province Colorado Plateaus

Tectonic Setting uplift

Terrane Definition

Gravity high (100x125km). Encompasses feature UTg18. Is contiguous with broader gravity high (AZg04). Corresponds with magnetic anomaly UTm21.

Hi/lo/both H

\section{Geologic Province}

Located over flat lying Pz and Mz strata of CRP. Outlines the Monument upwarp.

Inferred Source of Anomaly

May be dense and magnetic intrusive or uplifted buried basement rocks.

Generalized source rock intrusive mafic, basement mafic

References 


\section{UTg18}

Scale I

Province Colorado Plateaus

Tectonic Setting stable crust

Terrane Definition

Doughnut-shaped gravity high with internal low $(50 \mathrm{~km})$. Very good correlation with magnetic anomaly UTm22.

Hi/lo/both B

\section{Geologic Province}

Located over $\mathrm{Pz}$ and $\mathrm{Mz}$ flat lying strata of CRP, but corresponds with a small $\mathrm{T}$ intrusive outcrop (much smaller) that represents a deep seated intrusive or diatreme.

Inferred Source of Anomaly

May be dense magnetic mantle derived intrusive rocks.

Generalized source rock intrusive mafic, ultramafic

References

Mabey and others, 1978 


\section{UTm01

Province Great Basin

Tectonic Setting subduction, extension

\section{Terrane Definition}

Regional magnetic low lacking short-wavelength magnetic anomalies. Extends from the Walker Lane (NVm23) and Modoc Plateau (CAm02) anomalies on the west eastward across virtually the entire study area. Terrane extends from the SRP (IDm10) to the southern Quiet zone boundary (NVm29) and edge of the Colorado Plateaus (UTm12).

\section{Hi/lo/both L}

\section{Geologic Province}

Anomaly spans a wide range of rock types and ages.

\section{Inferred Source of Anomaly}

Speculation on source includes initial low magnetic susceptibilities of volcanic rocks (Stewart and others, 1977; Blakely, 1988), diminished magnetic susceptibility by intense hydrothermal alteration (Eaton, 1978). Demonstrated (Blakely, 1988) not to be the result of a shallow Curie isotherm.

Generalized source rock volcanic silicic, metamorphic

\section{References}

Stewart and others, 1977;

Eaton and others, 1978;

Mabey and others, 1978;

Blakely, 1988 


\section{UTm02 \\ Scale I}

Province Great Basin

Tectonic Setting hotspot

Terrane Definition

NW-trending magnetic high $(225 \times 50 \mathrm{~km})$. Extends SE from the eastern SRP. Corresponds with gravity anomaly UTg02.

Hi/lo/both H

\section{Geologic Province}

Located over the Great Salt Lake. Anomaly merges with the Great Rift that cuts across the eastern SRP. Has same trend as: $\mathrm{Pz}$ and $\mathrm{pC}$ basement outcrops, the Great Salt Lake, and the Great Rift. Located over $\mathrm{pC}$ bsement in the Raft River and Albion Mtns at its NW end.

\section{Inferred Source of Anomaly}

Possibly due to dense, magnetic mafic rocks associated with rifting. Has similar trend to basement on either side of eastern SRP. May be due to magnetic basement or to rift related intrusive rocks that were injected along pre-existing basement structure.

Generalized source rock intrusive mafic

References

Mabey and others, 1978;

Kuntz and others, 1988;

Kuntz and others, 1992 


\section{UTm03 \\ Scale I}

Province Middle Rocky Mtns, Great Basin

Tectonic Setting uplift

Terrane Definition

An oval (25km diameter) magnetic high located at the east end of the Uinta Mtns.

Hi/lo/both B

Geologic Province

Closely corresponds to pC outcrops of the Uinta Mtns.

Inferred Source of Anomaly

Magnetic structurally uplifted $\mathrm{pC}$ basement.

Generalized source rock basement mafic

References

Simpson and others, 1986 


\section{UTm04 \\ Scale I}

Province Great Basin

Tectonic Setting subduction, extension

Terrane Definition

Moderate NW-trending magnetic high $(75 \times 30 \mathrm{~km})$.

Hi/lo/both H

Geologic Province

Located over Q fill of Great Salt Lake Desert. Nearest outcrops are volcanic rocks and $\mathrm{pC}$ basement. Mz Gold Hill pluton lies to the south.

Inferred Source of Anomaly

Possibly due to magnetic pluton, magnetic basement, or volcanic rocks.

Generalized source rock intrusive mafic, basement mafic, volcanic mafic

References

Mabey and others, 1978 


\section{UTm05

Province Great Basin

Tectonic Setting subduction

\section{Terrane Definition}

ENE-trending narrow linear magnetic high (30x5km) west of boundary between Uinta Basin and Mtns (feature UTm05). Marks the southern edge of the Great Salt Lake Desert. Corresponds with gravity anomaly UTg04.

Hi/lo/both H

\section{Geologic Province}

Corresponds with several T intrusive rocks (Wasatch igneous belt) and stratified $\mathrm{Pz}$ sandstones. May be due to subduction-related volcanism along $\mathrm{pC}$ crustal discontinuity.

Inferred Source of Anomaly

Magnetic, low density intrusive rocks.

Generalized source rock intrusive silicic, sedimentary siliceous

References

Mabey and others, 1978;

Vogel and others, 2001 


\section{UTm06 \\ Scale I}

Province Middle Rocky Mtns, Great Basin,

Tectonic Setting depression

Terrane Definition

WNW-elongate oval-shaped magnetic high $(75 \times 40 \mathrm{~km})$ that lies within a gravity low. Corresponds with gravity anomaly $\mathrm{UTg} 07$.

Hi/lo/both H

Geologic Province

Corresponds with part of the Uinta Basin.

Inferred Source of Anomaly

Source is likely buried magnetic volcanic or intrusive rocks.

Generalized source rock sedimentary siliceous, volcanic mafic, intrusive mafic References 


\section{UTm07 \\ Scale}

Province Great Basin

Tectonic Setting subduction, extension

Terrane Definition

Elongate moderate magnetic high (50x25km).

Hi/lo/both H

Geologic Province

Located over outcrops of $\mathrm{pC}$ and $\mathrm{Pz}$ sedimentary, and $\mathrm{T}$ volcanic and intrusive rocks.

Inferred Source of Anomaly

Magnetic $\mathrm{Mz}$ and $\mathrm{T}$ intrusive rocks and/or T volcanic rocks.

Generalized source rock intrusive mafic, volcanic mafic

References

Mabey and others, 1978 


\section{UTm08}

Scale I

Province Great Basin

Tectonic Setting subduction, extension

Terrane Definition

EW-elongate magnetic high $(100 \times 40 \mathrm{~km})$. Located over a relative gravity low that is within a regional gravity high.

Hi/lo/both H

\section{Geologic Province}

Located over $\mathrm{pC}$ and Pz sedimentary and T volcanic rocks.

Inferred Source of Anomaly

May be due to magnetic basement and/or volcanic and associated intrusive rocks.

Generalized source rock basement mafic, volcanic mafic, intrusive mafic

References

Mabey and others, 1978 


\section{UTm09 salb 1}

Province Colorado Plateaus

Tectonic Setting depression

Terrane Definition

EW-elongate magnetic high $(125 \times 25 \mathrm{~km})$. Magnetic terrane overlaps with, but generally located south of gravity anomaly UTg09.

Hi/lo/both $\mathrm{H}$

Geologic Province

Located at southern edge of Uinta Basin. Anomaly parallels trend of Uinta Mtns.

Inferred Source of Anomaly

Magnetic high may reflect magnetic igneous rocks.

Generalized source rock intrusive mafic

References

Mabey and others, 1978 


\section{UTm10 \\ Scale I}

Province Colorado Plateaus

Tectonic Setting stable crust

Terrane Definition

Irregular magnetic high $(75 \times 75 \mathrm{~km})$ that includes magnetic highs UTm06,09, and an unlabeled high to the east. Anomaly spans several distinct EW-trending gravity highs and lows (UTg07,08,09).

Hi/lo/both H

Geologic Province

Located mostly over the Uinta Basin over T sedimentary rocks.

Inferred Source of Anomaly

Mostl likely due to magnetic basement rocks.

Generalized source rock basement mafic

References 


\section{UTm11 \\ Scale I}

Province Great Basin, Colorado Plateaus

Tectonic Setting subduction

Terrane Definition

Oval magnetic high $(75 \times 50 \mathrm{~km})$.

Hi/lo/both $\mathrm{H}$

Geologic Province

Located at north end of Sevier volcanic plateau.

Inferred Source of Anomaly

Magnetic volcanic rocks.

Generalized source rock volcanic mafic

References 


\section{UTm12}

Province Great Basin, Colorado Plateaus

Tectonic Setting stable crust, extension

Terrane Definition

Boundary between regional low of GB and regional high of CP.

Hi/lo/both B

Geologic Province

Boundary, in most places, skirts the CP.

Inferred Source of Anomaly

Marks the boundary between generally low magnetic terrain of the GB and the varied, but strongly magnetic terrain of the $\mathrm{CP}$. $\mathrm{CP}$ magnetic highs are largely due to zones of magnetic basement.

Generalized source rock basement mafic

References 


\section{UTm13}

\section{Scale}

Province Great Basin

Tectonic Setting subduction, extension

\section{Terrane Definition}

Elongate east-trending magnetic low $(50 \times 25 \mathrm{~km})$ with well defined northern and southern boundaries. Located over a gravity low.

\section{Hi/lo/both L}

\section{Geologic Province}

Southern edge of the anomaly corresponds with northern extent of a volcanic field bordering the $\mathrm{CP}$. Located over $\mathrm{Q}$ basin with some outcrops of $\mathrm{Pz}$ and $\mathrm{Mz}$ sedimentary rocks.

\section{Inferred Source of Anomaly}

Northern edge may reflect basement topography at bordering a Q basin. Southern edge defined by contrast of weakly magnetic basin fill with magnetic $T$ volcanic rocks.

Generalized source rock basement mafic, sedimentary siliceous, volcanic mafic 


\section{UTm14 \\ Scale I}

Province Colorado Plateaus

Tectonic Setting uplift

Terrane Definition

Arcuate NE-trending elongate magnetic high $(300 \times 40 \mathrm{~km})$. Corresponds with gravity anomaly UTm13.

Hi/lo/both H

\section{Geologic Province}

Roughly corresponds to the edge of the Wasatch plateau. Cuts across drainages of the CP. Located over part of the Circle Cliffs and San Rafel Swell structural uplifts.

Inferred Source of Anomaly

Likely reflects upwarp of dense, magnetic pC basement.

Generalized source rock basement mafic

References 


\section{UTm15 \\ Scale I}

Province Great Basin

Tectonic Setting subduction, extension

Terrane Definition

Elongate ENE-trending magnetic high (250x30km).

Hi/lo/both H

Geologic Province

Corresponds to Enterprise tholeitic basalts. Eastern part of anomaly corresponds with a $\mathrm{T}$ intrusive body.

Inferred Source of Anomaly

Strongly magnetic volcanic and intrusive rocks.

Generalized source rock volcanic mafic, intrusive mafic

References

Mabey and others, 1978;

Best and others, 1980 


\section{UTm16 \\ Scale $R$}

Province Colorado Plateaus

Tectonic Setting stable crust, uplift

Terrane Definition

Regional magnetic high of CP containing numerous large to intermediate magnetic highs (features UTm14, UTm17, UTm18, UTm19, UTm20, UTm21, UTm22, AZm01, AZm02, AZm05, AZm06, AZm07, AZm08), several of which are NE-trending.

Hi/lo/both H

\section{Geologic Province}

Largely located over flat lying Pz and Mz sedimentary rocks. Includes the La Sal Mtns $\mathrm{Cz}$ volcanic rocks. NE-trending anomalies corresponds to Colorado mineral belt.

Inferred Source of Anomaly

Regional high is most likely due to magnetic pC basement or $\mathrm{Cz}$ volcanic rocks.

Generalized source rock basement mafic, volcanic mafic

References

Case and Joesting, 1972;

Mabey and others, 1978;

Blank and others, 1998 


\section{UTm17 \\ Scale I}

Province Colorado Plateaus

Tectonic Setting uplift

Terrane Definition

NNE-trending elongate magnetic high (100x25km).

Hi/lo/both H

\section{Geologic Province}

Located over the northwestern CP over T and Mz sedientary rocks.

\section{Inferred Source of Anomaly}

May be similar to magnetic feature UTm14, possibly due to flexure and upwarp of magnetic $\mathrm{pC}$ basement rocks.

Generalized source rock basement mafic

References 


\section{UTm18}

Province Colorado Plateaus

Tectonic Setting stable crust, uplift

Terrane Definition

Oval magnetic high (25km).

Hi/lo/both H

Geologic Province

Located over the CP. Partly located over the Henry Mtns.

Inferred Source of Anomaly

Shape suggests it may be buried intrusive. Outcrops of Pz rocks suggest anomaly may also relate to an upwarp of $\mathrm{pC}$ basement rocks.

Generalized source rock intrusive mafic, basement mafic

References 


\section{UTm19 \\ Scale I}

Province Colorado Plateaus

Tectonic Setting uplift

Terrane Definition

Oval magnetic high $(75 \times 125 \mathrm{~km})$. Encompasses magnetic highs (magnetic features UT21 and UT22). Corresponds partly with gravity anomaly UTg17.

Hi/lo/both H

Geologic Province

Located over flat lying $\mathrm{Pz}$ and $\mathrm{Mz}$ strata of $\mathrm{CP}$ along the Monument upwarp.

Inferred Source of Anomaly

May be dense and magnetic intrusive or uplifted buried basement rocks.

Generalized source rock intrusive mafic, basement mafic

References 


\section{UTm20 \\ Scale I}

Province Colorado Plateaus, Great Basin

Tectonic Setting uplift

Terrane Definition

Elongate NNE-trending magnetic high that straddles the edge of a gravity high $(\sim 100 \times 25 \mathrm{~km})$.

Hi/lo/both H

\section{Geologic Province}

Located over the CP but close to Hurricane Fault that uplifts rocks to the east.

Inferred Source of Anomaly

May be due to magnetic $\mathrm{pC}$ basement rocks brought close to surface by Hurricane Fault.

Generalized source rock basement mafic

References 


\section{$\mathrm{UTm} 21$}

\section{Scale}

Province Colorado Plateaus

Tectonic Setting stable crust, uplift

Terrane Definition

Prominent magnetic high (50x50km). Adjoined with magnetic anomaly UTm22.

Hi/lo/both H

Geologic Province

Located over upper Pz carbonate and Mz clastic sedimentary rocks.

Inferred Source of Anomaly

May be due to magnetic intrusive or shallow pC basement rocks.

Generalized source rock intrusive mafic, basement mafic

References 


\section{UTm22 \\ Scale I}

Province Colorado Plateaus

Tectonic Setting stable crust

Terrane Definition

Doughnut-shaped magnetic high with internal low $(40 \mathrm{~km})$. Corresponds with gravity anomaly UTg18.

Hi/lo/both H

\section{Geologic Province}

Located over $\mathrm{Pz}$ and $\mathrm{Mz}$ flat lying strata of $\mathrm{CP}$, but corresponds with a small $\mathrm{T}$ intrusive outcrop (much smaller) that represents a deep seated intrusive or diatreme.

Inferred Source of Anomaly

May be dense magnetic mantle derived intrusive rocks.

Generalized source rock intrusive mafic, ultramafic

References

Mabey and others, 1978 


\section{WYg01 \\ Scale I}

Province Middle Rocky Mtns

Tectonic Setting uplift

Terrane Definition

Prominent NW-trending gravity high (200x30km). Corresponds with magnetic anomaly WYm02.

Hi/lo/both H

Geologic Province

Corresponds to Wind River Range.

Inferred Source of Anomaly

Dense, magnetic pC basement in the Wind River Range.

Generalized source rock basement mafic

References

Sims and others, 2001 


\section{WYg02 \\ Scale I}

Province Middle Rocky Mtns

Tectonic Setting depression

Terrane Definition

Very prominent NW-trending elongate gravity low $(175 \times 70 \mathrm{~km})$.

\section{Hi/lo/both L}

\section{Geologic Province}

Located over northern extension of Green River Basin. Bound on the south by a smaller amplitude gravity low. Bound on the NE by a prominent gravity high over the Wind River Mtns (WYg01).

Inferred Source of Anomaly

Low density basin fill.

Generalized source rock sedimentary siliceous

References 


\section{WYg03 \\ Scale I}

Province Middle Rocky Mtns

Tectonic Setting depression

Terrane Definition

Moderate north-trending gravity low (125x75km).

\section{Hi/lo/both L}

\section{Geologic Province}

Located over Cretacous sedimentary rocks over northern extension of Green River Basin. Bound on the south by prominent east-trending high of Uinta Mtns (UTg06). Bound on the east by prominent gravity high of the Rock Springs uplift (WYg04).

Inferred Source of Anomaly

Low density basin fill.

Generalized source rock sedimentary siliceous

References 


\section{WYg04 \\ Scale I}

Province Middle Rocky Mtns

Tectonic Setting uplift

Terrane Definition

North-trending gravity high $(125 \times 50 \mathrm{~km})$. Corresponds with magnetic anomaly WYm05.

Hi/lo/both H

Geologic Province

Rock Springs uplift -- a structural high that exposes Cretaceous sedimentary rocks.

Inferred Source of Anomaly

Dense and magnetic pC basement rocks at core of uplift.

Generalized source rock basement mafic

References

Sims and others, 2001 


\section{WYm01 \\ Scale}

Province Middle Rocky Mtns

Tectonic Setting depression

Terrane Definition

Variable magnetic region that is generally low $(>100 \mathrm{~km})$. Located over a gravity high. Southern portion overlaps with prominant gravity high (WYg01) associated with the Wind River Range.

Hi/lo/both L

Geologic Province

Located over large alluvial basin and over $\mathrm{pC}$ and $\mathrm{Pz}$ rocks.

Inferred Source of Anomaly

May be due to dense, and moderately to weakly magnetic $\mathrm{pC}$ basement and $\mathrm{Pz}$ sedimentary rocks.

Generalized source rock basement mafic, basement silicic, sedimentary siliceous

References

Sims and others, 2001 


\section{WYm02 \\ Scale I}

Province Middle Rocky Mtns

Tectonic Setting uplift

Terrane Definition

Prominent NW-trending magnetic high $(200 \times 30 \mathrm{~km})$. Corresponds with magnetic anomaly WYg01.

Hi/lo/both H

Geologic Province

Corresponds to Wind River Range.

Inferred Source of Anomaly

Dense, magnetic pC basement in the Wind River Range.

Generalized source rock basement mafic

References

Sims and others, 2001 


\section{WYm03 \\ Scale I}

Province Middle Rocky Mtns, Great Basin

Tectonic Setting uplift

Terrane Definition

Oval-shaped magnetic high $(75 \times 50 \mathrm{~km})$. Located over the western part of a gravity low, and over a basin filled with $\mathrm{T}$ sedimentary rocks and $\mathrm{Q}$ sediments.

Hi/lo/both H

\section{Geologic Province}

Located mostly over T sedimentary rocks and Q sediments.

Inferred Source of Anomaly

Buried magnetic pC basement.

Generalized source rock basement mafic

References

Sims and others, 2001 


\section{WYm04 \\ Scale I}

Province Great Basin, Middle Rocky Mtns

Tectonic Setting depression

Terrane Definition

Oval magnetic low $(60 \mathrm{~km})$. Surrounded partly by magnetic high feature WYm06.

\section{Hi/lo/both L}

\section{Geologic Province}

Located over Cretaceous sedimentary strata in Green River basin.

Inferred Source of Anomaly

Weakly magnetic pC metasedimentary rocks.

Generalized source rock basement silicic

References

Sims and others, 2001 


\section{WYm05 \\ Scale I}

Province Middle Rocky Mtns

Tectonic Setting uplift

Terrane Definition

North-trending low to moderate magnetic high (125×25km). Corresponds with magnetic anomaly WYg04.

Hi/lo/both H

Geologic Province

Rock Springs uplift -- a structural high that exposes Cretaceous sedimentary rocks.

Inferred Source of Anomaly

Dense and magnetic pC basement rocks at core of uplift.

Generalized source rock basement mafic

References

Sims and others, 2001 


\section{WYm06 \\ Scale I}

Province Middle Rocky Mtns, Great Basin

Tectonic Setting batholith, uplift

\section{Terrane Definition}

EW-elongate, U-shaped magnetic high $(175 \times 50 \mathrm{~km})$. Located north of Uinta Mtns, over alluvium and $\mathrm{T}$ sedimentary rocks in the Flaming Gorge area.

Hi/lo/both H

\section{Geologic Province}

Located over Cretacous sedimentary strata.

Inferred Source of Anomaly

Magnetic pC granitic rocks.

Generalized source rock intrusive silicic, intrusive mafic, basement mafic

References

Sims and others, 2001 